\title{
Magnetic Field Effects in Chemical Kinetics and Related Phenomena
}

\author{
ULRICH E. STEINER* and THOMAS ULRICH ${ }^{\dagger}$
}

Fakultät für Chemie, Universität Konstanz, D-7750 Konstanz, West Germany

\section{Contents}

I. Introduction

II. General Mechanistic Principles

A. A Unified Formal View

B. The Radical Pair Mechanism

C. Triplet-Triplet and Triplet-Doublet Pairs

D. The Triplet Mechanism

E. Paramagnetic Conversion of Ortho and Para Hydrogen

III. Experimental Methods

IV. Experimental Studies

A. In the Gas Phase

B. In the Solid State

C. In Homogeneous Liquid Solutions

1. Thermal Reactions

2. Photochemical Reactions

3. Photoluminescence and Chemiluminescence

4. Radioluminescence

D. In Micellar Solution

E. Interface Phenomena

F. Biological Systems

G. Reaction-Yield-Detected Magnetic Resonance

H. Magnetic Isotope Effects

V. Theoretical Studies

A. Mechanisms in the Gas Phase

B. The Triplet Mechanism

C. The Radical Pair Mechanism

1. Historical Roots

2. General Formalism

3. Spin Motion

4. The Dynamic Probability Function

5. Assembling Spin Motion, Diffusion, and Recombination

6. Treatments Based on Direct Solutions of Stochastic Liouville Equations

7. Time-Dependent Solutions of SLEs

D. Triplet-Triplet and Triplet-Doublet Pairs

E. Reaction-Yield-Detected Magnetic Resonance

F. Paramagnetic and Magnetocatalytic Ortho/Para-Hydrogen Conversion

VI. Conclusion

†Present address: Ilford AG, WFM 160.030, CH-1701 Fribourg, Switzerland.

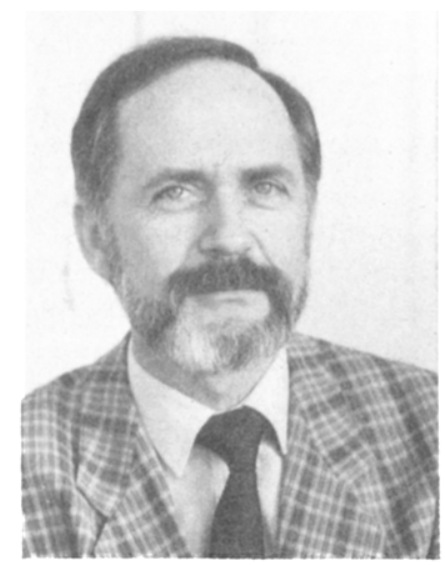

Ulrich E. Steiner was born in Backnang, Germany, in 1944. He studied chemistry in Stuttgart, where he obtained his Diplom degree in 1969, working with the late Th. Förster, and his Ph.D. in 1973, working with H. E. A. Kramer. He habilitated in 1979 at Stuttgart University. Before his appointment as Professor of Spectrochemistry at Konstanz University in 1981, he held a Heisenberg fellowship. His general research interests encompass elementary molecular reaction mechanisms in condensed phases, such as proton or electron transfer, cis-trans isomerization, and solvent effects on these processes. He is particularly interested in the role of electron spin during the elementary steps of photoelectron transfer reactions. His studies of magnetokinetic effects, dating back to 1976, were initiated by the latter problem.

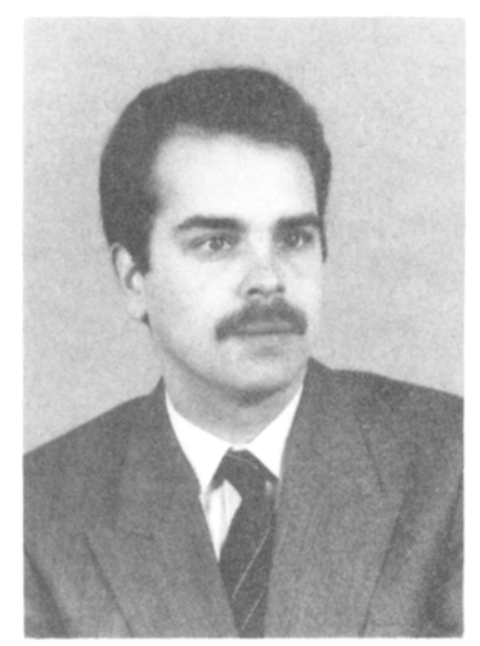

Thomas Ulrich was born in Calw, Federal Republic of Germany, in 1956. He studied chemistry at Stuttgart University and graduated in 1981, working with U. E. Steiner on the magnetokinetic triplet mechanism. In 1982 he joined U. E. Steiner's group at Konstanz University. His doctoral research work was devoted to the timeresolved investigation of magnetic field dependent radical pair kinetics in reverse micelles, for which he was awarded the BYK prize of the Herbert Quandt Stiftung in 1988. He received his Ph.D. in 1986. After 1 year of postdoctoral research work in Konstanz he is now a research scientist with Ilford AG in Fribourg, Switzerland. 


\section{Introduction}

Although the interest in experimental evidence of magnetic field effects (MFEs) on the kinetics of chemical reactions, which might be characterized by the term "magnetokinetics", has a long tradition, an impressive evolution of the field took place only after the discovery and understanding of nuclear and electronic spin polarization phenomena during chemical reactions (CIDNP, CIDEP) in the late $1960 \mathrm{~s}$. The so-called radical pair mechanism lying at the heart of these phenomena turned out to be a most valuable key for systematically tracing out MFEs on chemical yields and kinetics.

Nevertheless one should be aware that other mechanisms, too, with pairs of triplets, triplet-doublet pairs, or individual triplets, which originated at about the same time and were initially developed for explaining magnetic phenomena on luminescence in organic solids, also have their implication on chemical, particularly on photochemical, kinetics.

Phenomenologically, the basic mechanisms of magnetic-field-dependent reaction mechanisms may become apparent in fields and systems as different as the gas phase, the solid and liquid states, interfaces, and microheterogeneous systems such as micelles and in billogical systems. In all of these applications they have specific experimental and theoretical characteristics. Also, the techniques applied to study magnetokinetic phenomena span a large variety, ranging from magnetic resonance detection of spin polarization (CIDNP, CIDEP, ODMR) through "simple" detection of magneticfield-dependent reaction yields and magnetic isotope effects (MIE) to reaction-yield-detected magnetic resonance (RYDMR).

Thus the field of magnetokinetic chemical and related physical phenomena appears as a tree with several roots and many branches. Although each of these branches has been reviewed from time to time (cf. Table 1), most of the treatments have been rather specialized, and it is not easy to provide oneself with a broad and general view of the scope, objectives, and achievements of the field. Thus we have found it worthwhile to write this survey, developing the different aspects from a fairly general point of view (cf. section II), and to review, as comprehensively as possible, the original experimental (section IV) and theoretical (section V) work published since the early 1970 s, providing whenever possible a systematic compilation in the form of tables. Furthermore, in section III an outline of the various experimental techniques applied in the field is given.

Of course, the goals of completeness and compactness were not attainable without compromise. Thus the large field of chemically induced spin polarization phenomena would have been beyond the scope of this review. We have, however, attempted to include those theoretical papers in the field that have a general bearing on the understanding of magnetokinetic effects in general. We felt that, especially where photochemistry is concerned, the borderline between "truly" chemical and "purely" physical phenomena should not be defined too formally, since from the mechanistic and theoretical point of view they may be closely related. In order to account for this we included what has been termed "related phenomena" in the title of this review. Of course, the problem of delimitation cannot be solved without arbitrariness. The more photophysical aspects are mainly to be found in the sections on gas-phase and solid-state phenomena. In the solid state our attention has been mainly directed on work with organic molecular crystals. Only some representative references on inorganic solids and semiconductors are given.

We hope that this review may provide a welcome guide to the present body of literature on magnetokinetics, that it may help those working in the field to assess the achievements of current original work, and that it may be a useful framework of orientation for those who want to get into it or get an impression of the present scope of magnetokinetics.

\section{General Mechanlstic Principles}

\section{A. A Unified Formal View}

Early theoretical arguments in favor of magnetic field effects (MFEs) on chemical reactions were based on thermodynamics and suggested that chemical transformations should be accelerated by a magnetic field if they lead from diamagnetic to paramagnetic states, or vice versa. ${ }^{1}$ A quantitative estimation of magnetic contributions to the Gibbs free enthalpy shows, however, that no major effects are to be expected on these grounds. Using the electromagnetic cgs system with the magnetic induction $B_{0}$ in gauss $\left(=\mathrm{dyn}^{1 / 2} \mathrm{~cm}^{-1}\right)$, one may write the magnetic contribution $\Delta G_{\mathrm{m}}$ to the free enthalpy of reaction in a field of strength $B_{0}$ in a vacuum as

$$
\Delta G_{\mathrm{m}}=-(1 / 2) \Delta \chi_{\mathrm{M}} B_{0}^{2}
$$

where $\Delta \chi_{M}$ is the change of magnetic susceptibility during reaction of one molar unit. Assuming a rather high value of $10^{-2} \mathrm{~cm}^{3} / \mathrm{mol}$, which would correspond to production or consumption of about $5 \mu_{\mathrm{B}} \mathrm{mol}^{-1}$, one obtains in a field of $10000 \mathrm{G}$ (corresponding to $1 \mathrm{~T}$ ) $\Delta G_{\mathrm{m}}=0.05 \mathrm{~J} \mathrm{~mol}^{-1}$. At room temperature this means a change of the equilibrium constant by a factor of about $10^{-5}$. Thus, even in very high fields, magnetic field effects on chemical equilibria should be difficult to detect. 66

In discussing the possible implications of magnetic fields on the rates of chemical reactions, the general Eyring expression of absolute rate theory ${ }^{59}$ may be a more appropriate starting point:

$$
k=\left(k_{\mathrm{B}} T / h\right)_{\kappa} \exp \left(-\Delta G^{*} / R T\right)
$$

As far as the exponential term containing the free enthalpy of activation $\Delta G^{*}$ is concerned, its magnetic field dependence should be very weak as follows from the same arguments as used before. For adiabatic reactions (strong coupling between initial and final electronic states; cf. Figure 1) the transmission coefficient $\kappa$ approaches a value of 1 . Therefore, for this class of reactions we may exclude any significant magnetic field effect on the chemical reaction rate. In the case of diabatic reactions, however, i.e., where the electronic coupling between initial and final states does not provide for an efficient anticrossing of the respective potential curves, $\kappa$ may drop to rather small values, leaving in principle a large dynamic range

$$
-\infty \leq \log \kappa \leq 0
$$


TABLE 1. Reviews on Magnetokinetic Phenomena and Related Topics

\begin{tabular}{|c|c|c|c|c|}
\hline year & ref & topic ${ }^{a}$ & $\begin{array}{c}\text { no. of } \\
\text { refs }\end{array}$ & $\begin{array}{l}\text { no. of } \\
\text { pages }\end{array}$ \\
\hline 1929 & Bhatagnar et al. ${ }^{1}$ & MFD of reaction rates & 25 & 17 \\
\hline 1946 & Selwood ${ }^{2}$ & magnetism and catalysis & 222 & 42 \\
\hline 1967 & Figueras Roca ${ }^{3}$ & MFD of reaction rates & 70 & 7 \\
\hline 1968 & Avakian and Merrifield ${ }^{4}$ & triplet excitons & 116 & 41 \\
\hline \multirow[t]{3}{*}{1971} & Kaptein 5 & CIDNP $^{b}$ & $b$ & 206 \\
\hline & Buchachenko and Zhidomirov ${ }^{6}$ & CIDMP & 70 & 18 \\
\hline & Merrifield $^{7}$ & MFE on triplet excitons & 21 & 18 \\
\hline \multirow[t]{2}{*}{1972} & Ward ${ }^{8}$ & CIDNP & 32 & 7 \\
\hline & Lawler ${ }^{9}$ & CIDNP theory & 34 & 8 \\
\hline \multirow[t]{6}{*}{1973} & Lepley and Closs ${ }^{10}$ & $\mathrm{CIDMP}^{c}$ & $c$ & 416 \\
\hline & Lawler ${ }^{11}$ & CIDNP & 259 & 66 \\
\hline & Sokolik and Frankevich ${ }^{12}$ & MFE in organic solids & 131 & 28 \\
\hline & Swenberg and Geacintov ${ }^{13}$ & MFD of exciton interactions in organic solids & 207 & 76 \\
\hline & Glarum $^{14}$ & CIDNP theory & 39 & 39 \\
\hline & Atkins and McLauchlan ${ }^{15}$ & CIDEP theory & 96 & 53 \\
\hline 1974 & Avakian $^{16}$ & MFEs on triplet exciton interaction & 36 & 19 \\
\hline 1975 & Atkins and Lambert ${ }^{17}$ & MFEs on chemical reactions & 91 & 22 \\
\hline 1976 & Freed and Pedersen ${ }^{18}$ & CIDMP theory & 42 & 83 \\
\hline \multirow[t]{3}{*}{1976} & Buchachenko ${ }^{19}$ & MFEs on chemical reactions & 36 & 32 \\
\hline & Atkins and Evans ${ }^{20}$ & CIDEP theory & 78 & 29 \\
\hline & Faulkner ${ }^{21}$ & electrochemiluminescence & 135 & 51 \\
\hline 1977 & Sagdeev et al. ${ }^{22}$ & MFEs on reactions in solution & 95 & 33 \\
\hline \multirow[t]{2}{*}{1977} & Muus et al. ${ }^{23}$ & CIDMP $^{c}$ & $c$ & 303 \\
\hline & Küttner et al. ${ }^{24}$ & MFEs on gas-phase luminescence & 50 & 13 \\
\hline \multirow[t]{2}{*}{1978} & Geacintov and Swenberg ${ }^{25}$ & MFEs in organic molecular crystals & 103 & 60 \\
\hline & Selwood ${ }^{26}$ & magnetocatalytic $o / p \mathrm{H}_{2}$ conversion & 42 & 35 \\
\hline \multirow[t]{8}{*}{1979} & Hore and McLauchlan ${ }^{27}$ & time-resolved CIDEP & 53 & 17 \\
\hline & Pedersen ${ }^{28}$ & CIDMP theory ${ }^{b}$ & $b$ & 89 \\
\hline & Lin and Fujimura ${ }^{29}$ & MFEs on gas-phase luminescence & 87 & 44 \\
\hline & Adrian $^{30}$ & CIDMP theory & 75 & 41 \\
\hline & Molin et al. ${ }^{31}$ & MFEs on radical reactions in solutions & 98 & 66 \\
\hline & Schott $^{32}$ & MFEs at interfaces & 37 & 19 \\
\hline & Brocklehurst ${ }^{33}$ & MFEs in radioluminescence & 16 & 8 \\
\hline & Hoff ${ }^{34}$ & ESR in photosynthesis & 537 & 125 \\
\hline \multirow[t]{2}{*}{1980} & Wan $^{35}$ & CIDMP in photochemistry & 139 & 64 \\
\hline & Turro and Kräutler ${ }^{36}$ & MFEs and MIEs in organic photochemistry ${ }^{b}$ & 24 & 9 \\
\hline \multirow[t]{3}{*}{1981} & Hoff $^{37}$ & MFEs in photosynthesis & 80 & 67 \\
\hline & McLauchlan ${ }^{38}$ & MFEs on chemical reactions & 72 & 21 \\
\hline & Turro ${ }^{39}$ & MFEs and MIEs in micelles ${ }^{b}$ & 23 & 28 \\
\hline 1981 & Street 40 & spin-dependent effects in $a-\mathrm{Si}: \mathrm{H}$ & 130 & 83 \\
\hline \multirow[t]{4}{*}{1982} & Frankevich and Kubarev ${ }^{41}$ & RYDMR & 71 & 46 \\
\hline & Turro and Kräutler ${ }^{42}$ & ISC in diradicals & 56 & 61 \\
\hline & Schulten ${ }^{43}$ & MFEs in radical reactions ${ }^{b}$ & 19 & 23 \\
\hline & Hoff ${ }^{44}$ & ESR and ENDOR in photosynthesis & 254 & 43 \\
\hline \multirow[t]{4}{*}{1983} & Boxer et al. ${ }^{45}$ & MFEs in photosynthetic reaction centers & 62 & 29 \\
\hline & Brocklehurst ${ }^{46}$ & spin correlation in radiolysis & 44 & 10 \\
\hline & Turro and Weed ${ }^{47}$ & MFEs in micellar systems ${ }^{b}$ & 26 & 8 \\
\hline & Turro ${ }^{48}$ & MFEs and MIEs in organic radical reactions & 26 & 13 \\
\hline 1983 & Nagakura and Hayashi ${ }^{49}$ & MFEs on photochemical reactions in solution & 11 & 4 \\
\hline \multirow[t]{5}{*}{1984} & Buchachenko ${ }^{50}$ & MIEs and MI selection & 62 & 58 \\
\hline & Hoff ${ }^{61}$ & CIDEP in photosynthetic reaction centers & 236 & 130 \\
\hline & Salikhov et al. ${ }^{52}$ & CIDMP and MFEs in radical reactions ${ }^{e}$ & 511 & 419 \\
\hline & Gould et al. ${ }^{53}$ & MFEs and MIEs in organic reactions & 65 & 53 \\
\hline & Turro and Kräutler ${ }^{54}$ & MIEs in organic chemistry & 59 & 54 \\
\hline \multirow[t]{2}{*}{1985} & Buckley and McLauchlan ${ }^{55}$ & time-resolved CIDEP & 74 & 22 \\
\hline & Brocklehurst ${ }^{56}$ & spin correlation effects in radiolysis & 147 & 28 \\
\hline \multirow[t]{2}{*}{1986} & Hoff 57 & MFE and CIDEP in photosynthetic reaction centers & 160 & 29 \\
\hline & Maret et al. ${ }^{58}$ & biophysical $\mathrm{MFEs}^{\prime}$ & $f$ & 231 \\
\hline
\end{tabular}

a Abbreviations used: CIDNP (CIDEP, CIDMP), chemically induced dynamic nuclear spin (electron spin, magnetic) polarization; ISC, intersystem crossing; MFD, magnetic field dependence; MFE, magnetic field effect; MIE, magnetic isotope effect; RYDMR, reaction yield detected magnetic resonance. ${ }_{b}^{b}$ Review mainly on own work of corresponding authors. ${ }^{c}$ Multiauthor volume. d Monograph. ${ }^{e}$ Repeated citations in various chapters. 'Proceedings volume.

for magnetic field effects if these have any influence on the transmission factor $k$. From this one may deduce the principle that "diabaticity", i.e., a rather discontinuous change of the electronic wave function around the barrier, is a requirement for magnetically sensitive reactions.

For an appropriate theoretical treatment of such reactions one has to go beyond eq 2 and use explicit quantum theoretical expressions. The rate constant of irreversible decay of quantum states is usually based on the golden rule formula (cf., e.g., Freed's review on radiationless processes ${ }^{60}$, which may be stated as

$$
k_{i f}=(2 \pi / \hbar) \sum_{m} p_{m} \sum_{n}|\langle i, m|H| f, n\rangle|^{2} \delta\left(E_{i, m}-E_{f, n}\right)
$$

The symbols $i$ and $f$ denote initial and final electronic states, combined with vibrational manifolds $\{m\}$ and $\{n\}$, respectively. The population probabilities $p_{m}$ of the initial vibrational states are usually expressed by thermal equilibrium values. Equation 4 should be seen 


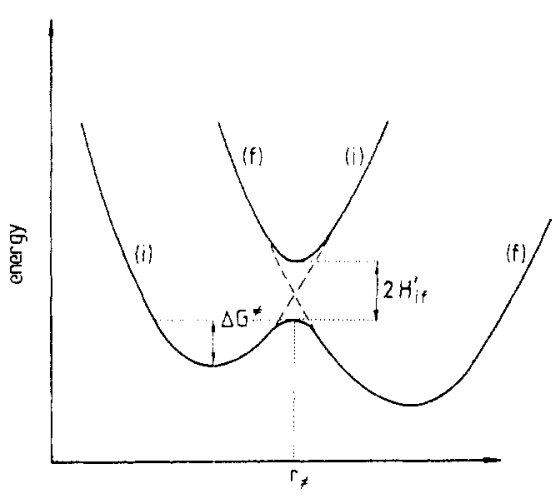

recuction coordinate, $r$

Figure 1. Schematic energy profiles for adiabatic and diabatic reactions. (i) and (f) denote unperturbed potential curves of initial and final electronic states. a)

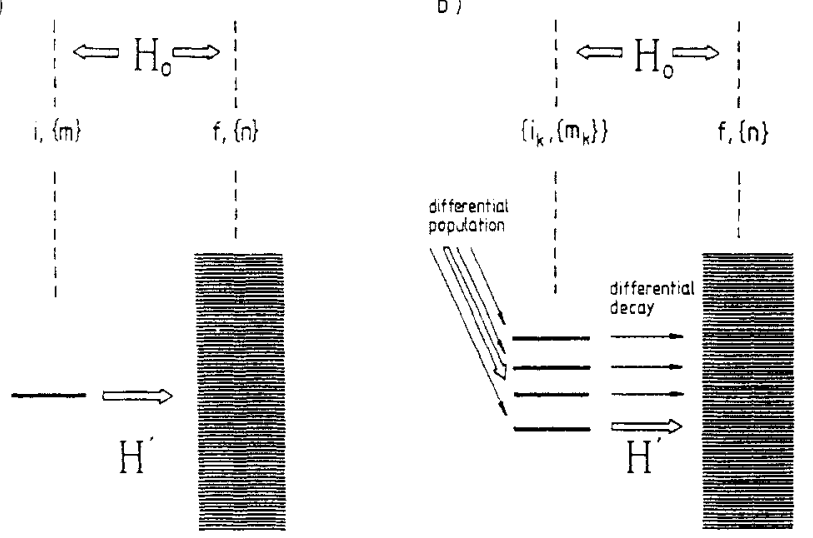

Figure 2. General schemes for explaining magnetic-field-dependent decay kinetics of electronic state $i$ : (a) decay of single electronic state; (b) decay of electronic manifold $\{i\}$. For details, cf. text.

in combination with Figure 2. Here, however, only one vibrational level of the initial state is indicated. It should be noted that the final vibrational manifold $\{m\}$ must form an effective quasi-continuum at the energy level of state $|i, m\rangle$ in order that truly irreversible decay can occur. The states $|i, m\rangle$ and $|f, n\rangle$ are experimentally observable quasi-stationary states and correspond to the eigenstates of a suitable Hamiltonian $H_{0}$. They become nonstationary through the perturbation $H^{\prime}$, whereby the rate of decay of the state $|i, m\rangle$ is proportional to the square of the corresponding coupling matrix elements with final states $|f, n\rangle$, and the $\delta$-function selects those final states that allow for conservation of energy.

For most situations in which magnetic field effects are observed scheme a of Figure 2 has to be extended to a scheme of type b. Now the initial situation comprises an electronic manifold $\left\{i_{k}\right\}$ of states with different electronic character, usually different spin states of the same orbital origin (of course, it is understood that each of the $\left(i_{k}\right)$ states is combined with a vibrational manifold $\left\{m_{k}\right\}$, but this will not be always explicitly indicated).

Sometimes it is possible to define an overall decay constant of the ensemble $\left\{i_{k}\right\}$ of initial states. Usually, however, one is content to obtain time-integrated yields into different decay channels. Such yields are always uniquely defined and are the quantities usually obtained from experiment.

Within the framework defined by the scheme in Figure $2 \mathrm{~b}$ the MFE on the decay kinetics originates through a magnetic contribution $H_{B}\left(\mathbf{r}, \sigma_{e}, \sigma_{N}\right)$ to the Hamiltonian $H$

$$
H=H_{0}+H^{\prime}
$$

where it may be included either in $H_{0}$, defining the quasi-stationary states of the system, or in $H^{\prime}$, inducing their decay. The magnetic interaction $H_{B}\left(\mathbf{r}, \sigma_{\mathrm{e}}, \sigma_{\mathrm{N}}\right)$ will generally depend on electronic coordinates $\mathbf{r}$ (through the contribution of orbital angular momentum) and on electronic $\left(\sigma_{\mathrm{e}}\right)$ and nuclear spin $\left(\sigma_{\mathrm{N}}\right)$ coordinates. If $H_{B}$ contributes to $H^{\prime}$, i.e., if

$$
H^{\prime}(B)=H^{\prime}(0)+H_{B}
$$

the magnetic field is directly responsible for the coupling between initial and final states, causing irreversible decay. This is the so-called direct mechanism as defined by Atkins and Stannard. ${ }^{61}$ An example is the case of magnetic predissociation of iodine (cf. sections IV and V).

The second, generally much more important, case includes $H_{B}$ as a constituent of $H_{0}$, which itself may be decomposed as

$$
H_{0}=H_{\text {st }}+H_{\text {rel }}
$$

where $H_{\mathrm{st}}$ denotes a time-independent part and $H_{\mathrm{rel}}$ a stochastically modulated contribution. The eigenstates of $H_{\mathrm{st}}$ are the observed quasi-stationary states $\left|i_{k}, m_{k}\right\rangle$ and $|f, n\rangle$. The effect of $H_{\text {rel }}$, which provides time-dependent couplings within these manifolds, is to cause relaxation within the manifolds. It may also account for collisional broadening of the $(f, n)$ levels in sparse manifolds of the small-molecule limit, where otherwise energy matching between $i$ and $f$ states, as required by the $\delta$-function, in eq 4 , would not be possible (collision-induced quenching).

The stationary part of $H_{0}$ may be decomposed as

$H_{\mathrm{st}}=H_{0}^{\mathrm{I}}(\mathbf{r}, \mathbf{R})+H_{0}^{\mathrm{II}}\left(\mathbf{r}, \sigma_{\mathrm{e}}, \sigma_{\mathrm{N}}\right)+H_{B}\left(\mathbf{r}, \sigma_{\mathrm{e}}, \sigma_{\mathrm{N}}, \mathbf{B}\right)$

where $H_{0}{ }^{\mathrm{II}}(\mathbf{r}, \mathbf{R})$ designates the electronic orbital and vibrational contribution, $H_{0}{ }^{\mathrm{I}}\left(\mathbf{r}, \sigma_{e}, \sigma_{\mathrm{N}}\right)$ is the nuclear and electronic spin Hamiltonian including spin-orbit coupling, and $H_{B}\left(\mathbf{r}, \sigma_{e}, \sigma_{N}, \mathbf{B}\right)$ is the Zeeman Hamiltonian depending on the magnetic field strength $\left(B_{0}\right.$ for a constant magnetic field or $B_{1} \sin (\omega t)$ for a microwave or radio-frequency magnetic field). The various electronic states of the $\left\{i_{k}\right\}$ manifold usually correspond to different eigenstates of the spin-dependent Hamiltonian $H_{0}^{\text {II }}+H_{B}$. However, they are usually orbitally and vibrationally degenerate, differing only in their electronic-nuclear spin wave functions.

The electronic spin manifold is usually realized as a molecular triplet state or as the spin manifold of paramagnetic reaction pairs such as of two triplets (nine spin states), triplet and doublet (six spin states), or two doublets (radical pair, four spin states). If $H_{B}$ contributes to $H_{0}$, magnetic effects are of the so-called indirect mechanism type (cf. Atkins and Stannard; ${ }^{61}$ our usage of the term is, however, more general). The indirect mechanism is based on the requirement that the coupling to the final manifold varies greatly among the states of the $\left\{i_{k}\right\}$ manifold (in many cases $k_{i f}$ even corresponds to the adiabatic limit for one of the initial states). Kinetic effects on the overall decay of the $\left\{i_{k}\right\}$ manifold ensue due to a magnetic recoupling, whereby the eigenstate basis $\left\{i_{k}\right\}_{B=0}$ is transformed to the new eigenstate basis $\left\{i_{k}{ }^{\prime}\right\}_{B}$. 
As will be exemplified below, all the specific models of mechanisms accounting for kinetic MFEs may be classified as subcases of the general concept outlined above. In order to do this systematically, the following distinctions are made.

(I) Coupling to the $\{f, n\}$ manifold: The matrix elements $H^{\prime}$ if may be (a) time independent or (b) stochastically modulated. In the reaction mechanism involving pairs of paramagnetic particles, time independence of $H^{\prime}$ usually requires fixed pair distances as in the solid state or solid-state-like situations. On the other hand, particle pairs that are free to diffuse, as in liquid solution, will undergo random encounters and in this way experience stochastic modulation of $H^{\prime}$.

(II) Initial population and redistribution in the $\{i\}$ manifold. (a) The systems entering the $\left\{i_{k}\right\}$ manifold may originate in states $\left|i_{k}{ }^{\prime}\right\rangle$ (e.g., in pure spin states) corresponding to coherent superpositions of the $H_{0}$ eigenstates $\left|i_{k}\right\rangle$, and it may be natural to describe the kinetics in terms of populations of the $\left|i_{k}{ }^{\prime}\right\rangle$, which will then exhibit a coherent time dependence determined by $H_{0}$. Therefore, in order to calculate specific yields, a time integration of this motion in $\left\{i_{k}{ }^{\prime}\right\}$, space must be performed. The most adequate method to do this and to also incorporate the effect of irreversible $i \rightarrow f$ transitions is the application of stochastic Liouville equations (SLEs).

(b) If rapid phase randomization occurs among the $\left|i_{k}\right\rangle$ components and if the effect of $H_{\text {rel }}$ is small, then the decay of the $\left\{i_{k}\right\}$ manifold corresponds to the $i \rightarrow$ $f$ transitions of kinetically isolated $H_{0}$ eigenstates $\left|i_{k}\right\rangle$, and the average reaction yields into various reaction channels are obtained as weighted sums of the individual $i_{k}$ contributions.

(c) If relaxation among the $\left|i_{k}\right\rangle$ due to $H_{\text {rel }}$ competes effectively with the $i \rightarrow f$ process, the overall decay may be described by a system of coupled kinetic equations for the populations of the $\left|i_{k}\right\rangle$ states.

(d) If relaxation in the $\left\{i_{k}\right\}$ manifold is fast with respect to the $i \rightarrow f$ decay, thermal equilibrium within the $\left\{i_{k}\right\}$ manifold will be established, and the whole population will decay as one quasi-state with a rate constant obtained by thermal averaging of the individual $\left(i_{k}\right)$ rate constants. It should be noted that magnetokinetic effects will ensue under such conditions only if the energy splittings within the $\left\{i_{k}\right\}$ manifold are at least comparable to, or larger than, $k T$. Otherwise the $\left\{i_{k}\right\}$ states will be equally populated and a recoupling of the $\left|i_{k}\right\rangle$ in a magnetic field will not change the average decay rate constant.

We now turn to a consideration of special mechanisms for magnetokinetic effects and establish their relation to the general framework described in this section.

\section{B. The Radical Pair Mechanlsm}

The principles of the radical pair mechanism have been explained in many original and review papers (cf. Table 1), among which especially the treatise by Salikhov et al..$^{52}$ must be mentioned. Therefore, except for introducing its basic features, we are mainly concerned here with demonstrating the relation of this mechanism to the general formalism described in the previnus section.

A typical situation considered in the radical pair mechanism is the production of a spin-correlated radical pair, let us assume from an electronically excited triplet state, yielding a radical pair with initially parallel electron spins. For energetic reasons the radical pair usually cannot recombine to yield an excited electronic state but can only form a diamagnetic ground-state recombination product that is of singlet spin multiplicity. Thus, direct recombination of the initial radical pair is spin-forbidden. However, the spin state of the radical pair is not stationary after separation of the two radicals, and spin evolution may eventually lead to a singlet correlated state of the electron spins, so that upon a next reencounter, the radical pair may recombine. This reaction (geminate recombination or cage recombination) competes with the final separation of the radicals (escape reaction with the possibility of forming products different from those of cage recombination). The ratio of cage to escape reaction yields will critically depend on the rate of spin evolution, which, on the other hand, depends on an external magnetic field. Thus the reaction kinetics becomes magnetic field dependent.

Comparing this model reaction mechanism with our previous general formalism, we note that the initial electronic state manifold corresponds to four electronic spin states of the radical pair, eventually combined with a manifold of nuclear spin states if these are to be explicitly considered:

$$
\begin{aligned}
&\left\{i_{k}\right\} \rightarrow\left\{\mathrm{RP}: \mathrm{S}, \mathrm{T}_{+}, \mathrm{T}_{0}, \mathrm{~T}_{-}\right\} \times\{\text {nuclear spin states }\} \\
&\left\{f_{1}\right\} \leftrightarrow\left\{\mathrm{CP}: \mathrm{S}_{0}\right\} \times\{\text { nuclear spin states }\} \\
&\left\{f_{2}\right\} \leftrightarrow\{\mathrm{EP}, \mathrm{SP}\} \times\{\text { nuclear spin states }\}
\end{aligned}
$$

There are two final electronic state manifolds corresponding to cage products $(\mathrm{CP})$ and escape or scavenge products (EP, SP), each combined with a manifold of nuclear spin configurations. Although the situation represented by eq 9 is fairly general, one should note that in a number of well-investigated cases, recombination products may be also formed in excited triplet states (cf. section IV.C).

The spin Hamiltonian $H_{0}$, which will be described in more detail in section $\mathrm{V}$, is usually a pure spin Hamiltonian. The part $H_{\mathrm{st}}$ determining the stationary states

$$
H_{\mathrm{st}}=H_{\mathrm{ihf}}+H_{\mathrm{ez}}\left(R_{\mathrm{ab}}\right)+H_{\mathrm{z}, \mathrm{el}}(B)
$$

comprises the isotropic hyperfine interaction $\left(H_{\mathrm{ihf}}\right)$, the interradical exchange interaction $\left(H_{\mathrm{ez}}\right)$, and the isotropic electronic Zeeman interaction $\left(H_{\mathrm{Z}, \mathrm{el}}\right)$. Since the exchange interaction depends strongly on the interradical separation $R_{\mathrm{ab}}$, this contribution is modulated by the diffusional motion of the radical pair. A rigorous theoretical account of this effect is one of the most difficult problems of radical pair theory.

Incoherent transitions among the eigenstates of $H_{\mathrm{st}}$ are induced by $H_{\text {rel }}$, comprising anisotropic hyperfine $H_{\text {rel }}=H_{\text {ahf }}+H_{\mathrm{az}, \mathrm{el}}(B)+H_{\mathrm{s} \text {-rot }}+$ other terms

interaction $\left(H_{\mathrm{ahf}}\right)$, anisotropic electronic Zeeman interaction $\left(H_{\mathrm{aZ}, \mathrm{el}}\right)$, spin-rotational interaction $\left(H_{\mathrm{g}-\mathrm{rot}}\right)$, and other interactions not explicitly mentioned. It must be noted, though, that spin relaxation in the radical pair, i.e., the contribution of $H_{\text {rel }}$, is neglected in most theoretical work on the radical pair mechanism. 


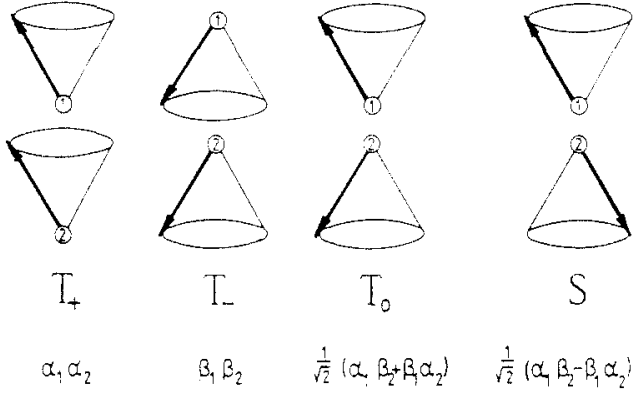

Figure 3. Vector representation of radical pair spin states $T_{+}$, $\mathrm{T}_{-}, \mathrm{T}_{0}$, and $\mathrm{S}$ (adapted from Turro and Kräutler ${ }^{42}$ ).

Within our general classification scheme the radical pair mechanism corresponds to a Ia/IIa case:

Coupling to the final states depends on the statistics of reencounters. Thus $H_{i f}^{\prime}$ is stochastically modulated. Furthermore, reactive coupling of $\left\{i_{k}\right\}$ to $\left\{f_{1}\right\}$ is a highly selective process. For the situation considered according to eq 9 , only the singlet radical pair state will couple to the cage product ground state.

The radical pairs originate in pure singlet or triplet spin states, when formed by chemical processes from precursors of the respective multiplicity, or else, when formed by free radical encounters, will assume this type of spin polarization due to multiplicity selection in a recombination process. In general, the pure spin states are not eigenfunctions of the Hamiltonian $H_{\mathrm{st}}$ and will consequently undergo coherent evolution in time. This process is termed "spin evolution".

In order to visualize this spin motion, it has become customary to use vector representations of the radical pair spin states ${ }^{8}$ (cf. Figure 3, with a representation adopted from Turro's work ${ }^{42,53}$ ). According to this picture the individual electron spins are confined to cones oriented along the axis of quantization, either upward ( $\alpha$-spin) or downward ( $\beta$-spin). The resultant of the two electron spins is either 1 , and oriented parallel $\left(T_{+}\right)$, perpendicular $\left(T_{0}\right)$, or antiparallel $\left(T_{-}\right)$to the axis of quantization, or $0(\mathrm{~S})$. The corresponding spin eigenfunctions are also given in Figure 3.

One should be careful not to overinterpret the graphical representation of $\mathrm{T}_{0}$ and $\mathrm{S}$. The figure suggests that there is some phase relation between the spins of different electrons. However, the phase relation distinguishing $\mathrm{S}$ and $\mathrm{T}_{0}$ operates between the spin function products $\alpha_{1} \beta_{2}$ and $\beta_{1} \alpha_{2}$, and not between the spins of single electrons. Actually, the picture used in Figure 3 for $\mathrm{S}$ and $\mathrm{T}_{0}$ would represent the spin function $\alpha_{1} \beta_{2}$, which is a spin-polarized state with a definite spin orientation on each radical. Its spin function corresponds to a superposition of $S$ and $T_{0}$. These pure spin states cannot be adequately represented with the simple two-vector model. Extended models using four vectors and giving a more consistent, though less obvious, representation of $S$ and $T_{0}$ have been used by Atkins ${ }^{62}$ and by Syage. ${ }^{63}$ Yet another model has been suggested by Monchick and Adrian, ${ }^{64}$ who transformed the equation of motion of the $\mathrm{S} / \mathrm{T}_{0}$ density matrix into a Bloch type equation of motion of a representative vector, the components of which are linear combinations of the density matrix elements. Of course, this type of vector is rather far from the initial intention to represent spins as vectors.

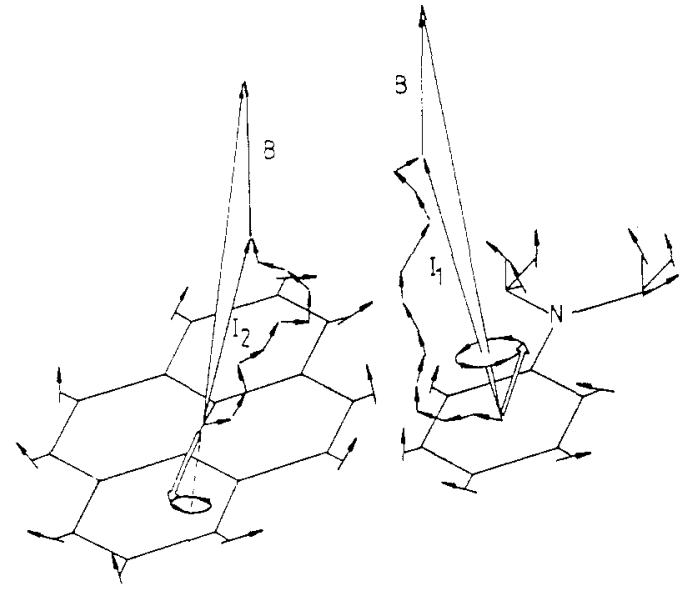

Figure 4. Graphical representation of electron spin precession in the resultant effective magnetic fields of local hyperfine interactions. Example of (pyrene $)^{*} /(N, N-\text { dimethylaniline })^{*+}$ radical pair. Reprinted from ref 65 with kind permission of $\mathrm{K}$. Schulten; copyright 1978 American Institute of Physics.

The main driving force for electronic spin motion is isotropic hyperfine coupling. Schulten and Wolynes ${ }^{65}$ have given an illuminating semiclassical description of this spin motion. It is based on the classical precession picture of angular momentum under a torque tending to align the angular momentum parallel to a given axis. The precession frequency (Larmor frequency) for the electron spins is given by

$$
\omega_{0}=g \mu_{\mathrm{B}} B / \hbar
$$

In the semiclassical picture the active magnetic field $\mathbf{B}$ is made up as a vector sum of the external magnetic field $\mathbf{B}_{0}$ and an effective magnetic field resulting from the sum of the hyperfine couplings of the various nuclear spins in the corresponding radical (cf. section V).

$$
\mathbf{B}=\mathbf{B}_{0}+\mathbf{B}_{\mathrm{hfc}}
$$

In this picture it is neglected that in the absence of a magnetic field the total spin angular momentum of electrons and nuclei must be conserved, so that a change in electron spin must be compensated by a change of nuclear spin. The effective nuclear hyperfine field is treated as a constant of motion in the semiclassical method, which is justified in the case of systems with many nuclear spins. Figure 4, from the work of Schulten and Wolynes, ${ }^{65}$ demonstrates the electron spin motion in a specific radical pair. Generally, the electron spins in the two radicals of a pair precess about different axes and at different frequencies. Thus, the relative orientation of the two electron spins changes in time between parallel and antiparallel alignment. In zero external field any transition between the spin substates of the radical pair is possible. As the external field strength increases, the resultant field $\mathbf{B}$ is more and more determined by $\mathbf{B}_{0}$ so that the directions of the precession axes of the two spins coincide, precluding transitions between $T_{+}, T_{-}$and $S, T_{0}$ (so-called spin-flip transitions). However, the precession frequency difference due to the $\mathbf{B}_{\mathrm{hfc}}$ component parallel to $\mathbf{B}_{0}$ is retained, and $\mathrm{S}-\mathrm{T}_{0}$ transitions (so-called rephasing transitions) are not suppressed by the external magnetic field.

It should be noted that precession frequency differences may arise not only from different $B$ values at the 


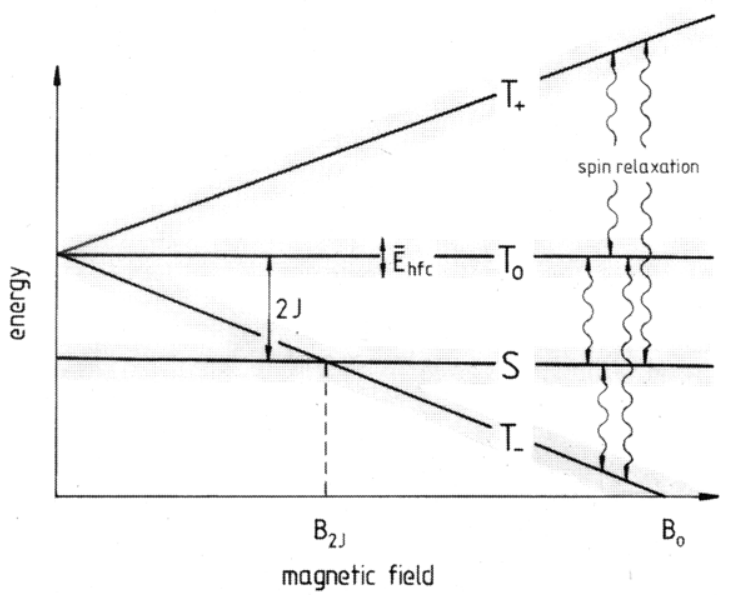

Figure 5. Energy diagram of electronic spin states of a radical pair in a magnetic field. $\bar{E}_{\mathrm{hfc}}$ is an average bandwidth due to hyperfine coupling, and $J$ is the exchange integral.

two spin sites but also from different $g$ factors (referred to as the $\Delta g$ mechanism or sometimes the Zeeman mechanism). The latter contribution affords $\mathrm{T}_{0}-\mathrm{S}$ transitions occurring at a rate that increases linearly with the magnetic field. Thus, MFEs due to the $\Delta g$ mechanism and those due to the hyperfine mechanism have opposite signs.

The semiclassical picture also gives an idea of the effect of increasing exchange interaction. This interaction causes electron spin exchange at a frequency of

$$
\omega_{\mathrm{ex}}=2 J / \hbar
$$

If this frequency of exchange exceeds the individual Larmor frequencies, each spin will experience the same average Larmor frequency, so that any difference between the electron spin motions at different radicals disappears and no realignment will occur.

The singlet-triplet conversion mechanism will be also conveniently expressed in terms of an energy diagram (cf. Figure 5). Here each of the spin states is given a certain width, corresponding to the average of the isotropic hyperfine coupling strength. Singlet-triplet transitions may occur only if singlet and triplet sublevels approach each other within this width. Thus, if $J$ is zero, there will be unrestricted singlet-triplet mixing at zero field, but as the Zeeman splitting increases $\left(E_{\mathrm{Z}}>E_{\mathrm{hfc}}\right)$, the outer Zeeman components $\mathrm{T}_{+}$ and $\mathrm{T}_{-}$will be cut off from the conversion process, and the $\mathrm{T}_{0} \rightarrow \mathrm{S}$ process will be left at high field. If, however, $2 J>E_{\mathrm{hfc}}$, no singlet-triplet transitions will be possible at zero field. A triplet-singlet level crossing will occur, however, at some higher field, allowing for triplet-singlet transitions, which at still higher fields are suppressed again. This situation should be expected to give a type of resonance in the field dependence, with a maximum at a magnetic field of $B_{0}=2 \mathrm{~J} / \mathrm{g} \mu_{\mathrm{B}}$.

Although, within the picture of coherent spin motion, $\left(\mathrm{T}_{+}, \mathrm{T}_{-}\right) \rightarrow \mathrm{S}$ transitions are suppressed at high magnetic fields, the stochastically modulated contribution of $H_{\text {rel }}$ will cause incoherent (relaxational) transitions among the spin substates. Their rate will, in general, also depend on the Zeeman splitting of the sublevels. Singlet-triplet transitions induced by $H_{\text {rel }}$, though generally much slower than those induced by $H_{\text {ihf }}$, may also influence the overall kinetics of chemical reactions if the lifetime of correlated pairs is long enough to probe such
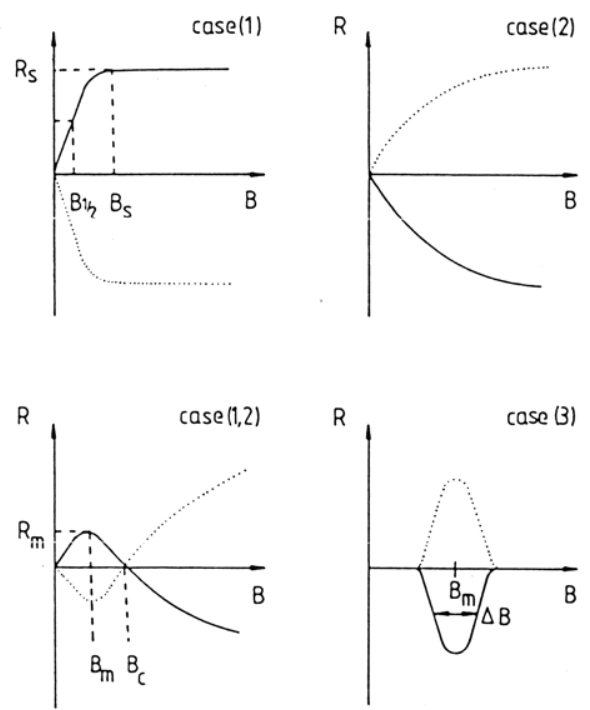

Figure 6. Phenomenological cases of MFD of reaction yields (adapted from Sakaguchi et al. ${ }^{67}$ ). Dotted lines indicate sign inversion of effects when changing the precursor multiplicity in the radical pair mechanism. For details, cf. text.

relaxational transitions. Since these, too, will in general depend on the magnetic field strength, they can also give rise to magnetic-field-dependent chemical yields and kinetics (cf. MFEs in micellar systems, section IV.D).

The magnetic field dependence (MFD) of chemical reaction yields caused by the radical pair mechanism may be classified into three cases or combinations of them. ${ }^{53,67}$ In Figure 6 are depicted the cases proposed by Hayashi et al. ${ }^{67}$ with some changes in the notation. Case 1 is typical of the suppression of the hyperfinecoupling-induced $\mathrm{S} \leftrightarrow \mathrm{T}_{+,-}$transitions by the magnetic field. It is characterized by a clear saturation behavior. Parameters to be specified may be $B_{1 / 2}$, the field where half of the saturation effect is obtained, and $B_{\mathrm{s}}$, the region of beginning saturation, although the latter is not very exactly defined. Curves of this type are also obtained in the case of the so-called relaxation mechanism, however with larger $B_{1 / 2}$ and $B_{\mathrm{s}}$ values.

Case 2, characterized by a rather monotonously rising MFD curve, is typical of the $\Delta g$ mechanism, which requires very high magnetic fields for obtaining a saturation limit. ${ }^{68}$ The combined appearance of case 1 and case 2 may lead to MFD curves with minima or maxima and sign inversion due to the counteracting influence of both mechanisms, each of which dominates in a certain region of the magnetic field (case 1,2). Such curves may be characterized by the field values $B_{\mathrm{m}}$ of the maximum and $B_{\mathrm{c}}$ of the zero-line crossing.

The case 3 situation would be ideally encountered with radical pairs subject to a moderate but constant exchange interaction $\left(2 J>E_{\mathrm{hfc}}\right)$. It is typical for a level-crossing situation and may be characterized by the field values $B_{\mathrm{m}}$, corresponding to the maximum, and $\Delta B$, corresponding to the width of the resonance.

\section{Triplet-Triplet and Triplet-Doublet Pairs}

A number of processes are known in molecular crystals and liquid solutions where pairs of two triplets or a triplet and a doublet interact by transferring electronic energy, involving a change of local multipl- 
icity. Thus, triplet-triplet annihilation (in crystals also called triplet exciton fusion) may lead to higher excited singlets, a mechanism producing delayed fluorescence (eq 15).

$$
\mathrm{T}+\mathrm{T} \rightleftarrows(\mathrm{TT}) \rightarrow \mathrm{S}^{*}+\mathrm{S}_{0}
$$

The reverse of this process is also possible (so-called singlet exciton fission: of. eq 16),

$$
\mathrm{S}^{*}+\mathrm{S}_{0} \rightleftarrows(\mathrm{TT}) \rightarrow \mathrm{T}+\mathrm{T}
$$

whereby a sometimes "hot", i.e., vibrationally excited, singlet combines with an adjacent ground-state molecule to yield a pair of two triplets, which may eventually separate. This process contributes to the quenching of prompt fluorescence.

The interaction between a triplet and a doublet species may lead to triplet deactivation with dissipation of electronic excitation energy or, eventually, electronic excitation of the doublet molecule (eq 17). Processes of this type may be responsible for triplet quenching in liquid solution or for mobilization of trapped charge carriers in molecular crystals.

$$
\left.\mathrm{T}+\mathrm{D} \rightleftarrows(\mathrm{TD}) \rightarrow \mathrm{S}_{0}+\mathrm{D} \text { (or } \mathrm{D}^{*}\right)
$$

Several excellent reviews on these processes and their mechanisms have appeared.,13,16,25 Therefore, besides giving a short outline of the basic mechanism, we have as our main object its correlation with the general mechanistic view introduced in section $A$.

MFEs on the kinetics of the processes described by eq 15-17 are of the indirect type. The initial state manifold $\left\{i_{k}\right\}$ consists of the set of coupled spin states of the pair (nine states in the case of a TT pair and six in the case of a TD pair). The way the $\left\{i_{k}\right\}$ states are coupled together to energy eigenstates depends on the strength and direction of an external magnetic field. On the other hand, the reactivities of the pairs into various product channels are governed by the principle of total-spin conservation. In terms of our general classification scheme the mechanistic type according to which these effects are usually treated corresponds to the $\mathrm{Ia} / \mathrm{IIb}$ case or sometimes to the Ia/IIa case.

The interaction Hamiltonian $H^{\prime}$, inducing reactions of the pair, is usually considered as constant during the lifetime of the pair and treated semiempirically by attributing first-order rate constants to the spin-allowed reaction channels.

Interactions contributing explicitly to $H_{0}$ are

$$
H_{0}=H_{\mathrm{ZFS}}+H_{\mathrm{ex}}+H_{\mathrm{Z}}(B)
$$

where $H_{\mathrm{ZFS}}$ is the zero field splitting Hamiltonian accounting for intramolecular spin-spin and spin-orbit interaction in the triplet molecules. It is conveniently expressed by using the ZFS tensors $\mathbf{D}_{1}$ and $\mathbf{D}_{2}$ :

$$
H_{\mathrm{ZFS}}=\boldsymbol{S}_{1} \cdot \mathbf{D}_{1} \cdot \boldsymbol{S}_{1}+\boldsymbol{S}_{2} \cdot \mathbf{D}_{2} \cdot \boldsymbol{S}_{2}
$$

In the case of a doublet in the pair, one of the ZFS terms should be substituted by the isotropic hyperfine interaction, which, however, is of lower order of magnitude.

The intermolecular electron exchange interaction $H_{\mathrm{ex}}$ is often neglected in theoretical treatments of TT and TD pairs (for exceptions cf. ref 69 and 70). Also neglected is intermolecular spin-spin interaction (not explicitly appearing in eq 18). The final term, $H_{\mathrm{Z}}(B)$ is the electronic Zeeman Hamiltonian.
In terms of products of the individual triplet highfield eigenstates $|0\rangle,|+\rangle$, and $|-\rangle$ the coupled spin eigenstates of a TT pair are given by ${ }^{7}$

$$
\begin{gathered}
|\mathrm{S}\rangle=(1 / \sqrt{3})(|00\rangle-|+-\rangle-|-+\rangle) \\
\left|\mathrm{T}_{+1}\right\rangle=(1 / \sqrt{2})(|+0\rangle-|0+\rangle) \\
\left|\mathrm{T}_{0}\right\rangle=(1 / \sqrt{2})(|+-\rangle-|-+\rangle) \\
\left|\mathrm{T}_{-1}\right\rangle=(1 / \sqrt{2})(|-0\rangle-|0-\rangle) \\
\left|\mathrm{Q}_{+2}\right\rangle=|++\rangle \\
\left|\mathrm{Q}_{+1}\right\rangle=(1 / \sqrt{2})(|+0\rangle+|0+\rangle) \\
\left|\mathrm{Q}_{0}\right\rangle=(1 / \sqrt{6})(2|00\rangle+|+-\rangle+|-+\rangle) \\
\left|\mathrm{Q}_{-1}\right\rangle=(1 / \sqrt{2})(|-0\rangle+|0-\rangle) \\
\left|\mathrm{Q}_{-2}\right\rangle=|--\rangle
\end{gathered}
$$

In the case of negligible intermolecular interactions the pair energy eigenstates are simple products of the individual triplet energy eigenstates. In general, these are not identical with the pair spin eigenstates of eq 20 , but linear combinations of these. One should note, however, that in case of $\mathbf{D}_{1}=\mathbf{D}_{2}$ the spin Hamiltonian is symmetric with respect to exchange of the triplet spin operators $\boldsymbol{S}_{1}$ and $\boldsymbol{S}_{2}$, so that the energy eigenstates may be classified as symmetric or antisymmetric, respectively. As follows from eq 20 , the pair triplet states are the antisymmetric ones, whereas the pair singlet and quintet states are symmetric with respect to spin exchange. This separation will be valid at any field strength so that, in this case, the singlet pair state may be distributed over at most six energy eigenstates.

In comparison to their width, determined by the lifetime of the correlated TT or TD pairs, the eigenstates of $H_{0}$ are normally clearly separated. Thus, overall reaction efficiencies $\gamma_{\mathrm{TT}}$ and $\gamma_{\mathrm{TD}}$ may be determined (case IIb) as the average of contributions of single energy levels, for each of which the reaction rate constant is proportional to the singlet character $S_{i}$ or doublet character $D_{i}$, respectively, of the $H_{0}$ eigenstate:

$$
\begin{aligned}
\gamma_{\mathrm{TT}} & =\frac{1}{9} \sum_{i} \frac{k_{\mathrm{S}} S_{i}}{k_{-1}+k_{\mathrm{S}} S_{i}} \\
\gamma_{\mathrm{TD}} & =\frac{1}{6} \sum_{i} \frac{k_{\mathrm{D}} D_{i}}{k_{-1}+k_{\mathrm{D}} D_{i}}
\end{aligned}
$$

Here, $k_{\mathrm{S}}$ and $k_{\mathrm{D}}$ are the reaction rate constants for pure singlet or doublet pairs, and $k_{-1}$ is the rate constant of dissociation of such pairs. The expressions are kinetically obvious.

This kind of treatment is due to Merrifield. ${ }^{71}$ It follows from eq 21 (analogous conclusions may be drawn from eq 22 for TD pairs) that redistribution of singlet character $S_{i}$ over the various eigenstates $\left|i_{k}\right\rangle$ as occurring under the influence of a magnetic field will change the overall efficiency $\gamma_{\mathrm{TT}}$ of the reaction. One may draw the general conclusion that $\gamma_{\mathrm{TT}}$ is larger the more uniform $S_{i}$ is distributed among the eigenstates. This may be demonstrated with the extreme cases $S_{i}=1 / 9$, 
TABLE 2. Distribution of Singlet Character over TT-Pair Energy Eigenstates

\begin{tabular}{ccc}
\hline $\begin{array}{c}\text { magnetic } \\
\text { field }\end{array}$ & \multicolumn{2}{c}{$\begin{array}{c}\text { no. of energy eigenstates } \\
\text { carrying singlet character }\end{array}$} \\
\cline { 2 - 3 } & $\mathbf{D}_{1}=\mathbf{D}_{2}$ & $\mathbf{D}_{1} \neq \mathbf{D}_{2}$ \\
\hline$B_{0}=0$ & 3 & 9 \\
$B_{0} \leq$ ZFS & $<3^{a}$ & $<9^{a}$ \\
$B_{0} \gg$ ZFS & $(1),{ }^{b} 2$ & $(1),^{b} 2$
\end{tabular}

a Precise value depends on field orientation; in solids extrema are found at directions called "low-field resonances". "In solids at special field orientations, where the so-called "high-field resonances" are observed.

i.e., completely uniform distribution, and $S_{i}=\delta_{i, 1}$, i.e., concentration of all singlet character on one eigenstate only. The results (eq 23 and 24) show that the change in $\gamma$ may correspond to a factor of 9 in the favorable case where $k_{\mathrm{S}} \gg k_{-1}$, i.e., in the case of very efficient singlet pair reaction.

$$
\begin{aligned}
& \gamma_{\mathrm{TT}}\left(S_{i}=1 / 9\right)=\frac{1}{9} \frac{k_{\mathrm{S}}}{k_{-1}+1 / 9 k_{\mathrm{S}}} \\
& \gamma_{\mathrm{TT}}\left(S_{i}=\delta_{i, 1}\right)=\frac{1}{9} \frac{k_{\mathrm{S}}}{k_{-1}+k_{\mathrm{S}}}
\end{aligned}
$$

The dilution principle of most efficient recombination has been nicely visualized in a hydrodynamic model by Avakian. ${ }^{16}$ It is based on the fact that as the recombination rate constants increase, the recombination efficiencies approach a saturation limit of 1 . Other examples where this principle works are the triplet mechanism (cf. section D) and a related mechanism explaining magnetic fluorescence quenching of glyoxal. ${ }^{172}$

Phenomenologically, typical MFD curves for effects with triplet-triplet pairs correspond to case 1,2 of Figure 6. As to be expected, fusion and fission processes have identical magnetic field dependence, however with inverted signs (cf. Figure 10). This MFD may be qualitatively understood in terms of the singlet dilution principle as documented in Table 2 . In the case of $\mathbf{D}_{1}$ $=\mathbf{D}_{2}$, i.e., for homofission or -fusion with parallel orientation of the interacting triplet excitons, only three zero-field pair eigenstates carry singlet character. This number increases with an external magnetic field oriented at an arbitrary angle to the ZFS tensor axes. The increase of singlet dilution causes an increase of the triplet-triplet annihilation efficiency. At fields $B_{0} \gg$ $D$ the singlet character is in general restricted to two energy eigenstates, which means that the reaction efficiency drops below the value at zero field. Tripletdoublet interactions do not lead to the initial maximum displayed in Figure 10. Such a maximum is also not to be expected for triplet-triplet interactions in liquid solutions, where, due to their random orientation, $\mathbf{D}_{1}$ $\neq \mathbf{D}_{2}$ even for two triplets of the same kind. Maxima may occur, however, also in liquid solution if considerable exchange interaction comes into play ${ }^{69}$ (cf. section V).

In the solid state the pair eigenstates are dependent not only on the strength of the magnetic field but also on its orientation with respect to the crystal axes. If, e.g., at low field, the field direction is parallel to any of the fine-structure axes, the singlet character will be concentrated on a minimum of eigenstates, meaning that a resonance minimum is detected when the orientation of the crystal is swept over such a position (lowfield resonances).

In high fields the singlet character is generally distributed over two energy eigenstates:

$$
\begin{aligned}
&\left|(\mathrm{SQ})_{0}\right\rangle=|00\rangle=(1 / \sqrt{3})|\mathrm{S}\rangle+(\sqrt{2} / \sqrt{3})\left|\mathrm{Q}_{0}\right\rangle \\
&\left|(\mathrm{SQ})_{1}\right\rangle=(1 / \sqrt{2})(|+-\rangle+|-+\rangle)= \\
&(-\sqrt{2} / \sqrt{3})|\mathrm{S}\rangle+(1 / \sqrt{3})\left|\mathrm{Q}_{0}\right\rangle
\end{aligned}
$$

These have $1 / 3$ or $2 / 3$ singlet character, respectively. Scanning, however, the orientation of the crystal with respect to the magnetic field, a level crossing may occur between these. At the position of the crossing the treatment according to case IIb is no longer justified; i.e., the decay of these states has to be treated in a coupled manner. It has been shown ${ }^{73}$ that exactly at the resonance position the system behaves as if the singlet character is concentrated on one state only. These resonances are the so-called high-field resonances (cf. Figure 11).

\section{The Triplet Mechanism}

This mechanism, too, belongs to the class of indirect mechanisms, where the magnetic field does not directly contribute to the interaction inducing the reactive decay of the initial state. Instead, the magnetic field recouples the manifold of initial states, in this case the substates of an electronically excited triplet state:

$$
\left\{i_{k}\right\}=\left\{\mathrm{T}_{\mathrm{u}}, \mathrm{T}_{\mathrm{c}}, \mathrm{T}_{\mathrm{l}}\right\}
$$

where $u, c$, and $l$ stand for the upper, central, and lower substates. These are eigenstates of $H_{0}$, given as

$$
H_{0}=H_{\mathrm{ZFS}}(\Omega)+H_{\mathrm{Z}}(\mathbf{B})
$$

where the $\Omega$ argument of the ZFS Hamiltonian emphasizes the orientational dependence of this interaction in a laboratory-fixed frame of reference. For molecules in liquid solution it is subject to random modulation due to rotational diffusion of the molecules. Nevertheless, one cannot subsume it under $H_{\text {rel }}$ because the reactive perturbation $H^{\prime}$, given by

$$
H^{\prime}=H_{\mathrm{so}}(\Omega)+H_{\text {chem }}^{\prime}
$$

is also subject to a random modulation that is strictly correlated with the modulation of $H_{\mathrm{ZFS}}$. $H^{\prime}$ is composed of the spin-orbit coupling operator $H_{\mathrm{so}}$, which is responsible for triplet-sublevel-selective intersystem crossing processes. Like $H_{\mathrm{ZFS}}$, it is also defined in the molecular frame of reference and hence randomly modulated in the same way as $H_{\mathrm{ZFS}}$ if the molecular states are described in a laboratory-fixed frame of reference. A convenient basis of the latter would be given by the energy eigenstates in high magnetic fields.

The perturbation operator $H^{\prime}$ may also entail an operator $H_{\text {chem }}^{\prime}$ responsible for the coupling to chemical reaction channels that compete with $\mathrm{T} \rightarrow \mathrm{S}_{0}$ intersystem crossing. In general, $H_{\text {chem }}^{\prime}$ is not spin-sublevel selective (for possible exceptions, cf. ref 74 and 75 ). However, its competition with the spin-sublevel-selective $H_{\mathrm{so}}$ provides the basis for MFEs on the chemical reaction yields. A main difference of the triplet 


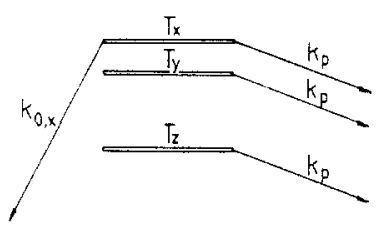

a)

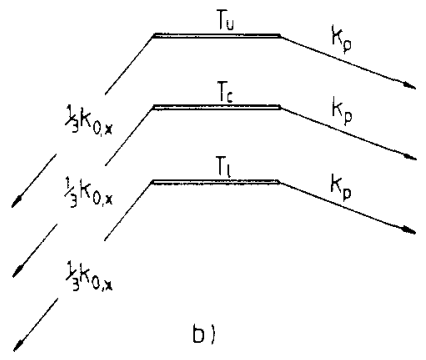

Figure 7. A simplified triplet-sublevel-selective reaction scheme explaining the principle of how MFEs on product yields follow from the triplet mechanism: (a) zero-field situation, a special case of selective ISC of one substate only; (b) magnetic mixing of zero-field spin states distributes ISC over all energy eigenstates in the magnetic field (the uniform distribution depicted is a special case).

mechanism as compared to the pair mechanisms is that the sublevel-selective process is not multiplicity conserving in the triplet mechanism, whereas it is in the pair mechanisms.

Whereas triplet-sublevel-selective processes have been known since about 1965 from phosphoresecence studies at low temperatures, ${ }^{76-78}$ sublevel-selective triplet population was recognized as a source of electron spin polarization in CIDEP by Atkins and McLauchlan ${ }^{15}$ and by Wong et al. ${ }^{79}$ in 1973 . It must be pointed out, however, that spin-selective population of triplet sublevels in itself is neither necessary nor sufficient to explain MFEs on chemical reaction yields. Therefore the aspect of selective population will not be considered in the following.

A consistent theoretical treatment of the triplet mechanism corresponds to a Ib/IIa case in the classification scheme of section $\mathrm{A}$. The random modulation of $\Omega$ in $H_{0}$ and $H^{\prime}$ makes it difficult, however, to translate such a treatment into a simple picture. Therefore, in order to obtain some direct insight, we shall consider a very simplified situation, namely, all triplet molecules fixed and oriented parallel with their ZFS tensor axes. Consider the situation depicted in Figure 7, where in zero field only one of the substates $\left(\mathrm{T}_{x}\right)$ shall undergo radiationless decay by intersystem crossing to $\mathrm{S}_{0}$ (rate constant $k_{0, x}$ ), whereas from all triplet substates some product channel may be open with a rate uniform constant $k_{\mathrm{p}}$. In a magnetic field the substates are recoupled as follows:

$$
\left\{\mathrm{T}_{x}, \mathrm{~T}_{y}, \mathrm{~T}_{z}\right\}_{\mathrm{zF}} \rightarrow\left\{\mathrm{T}_{\mathrm{u}}, \mathrm{T}_{\mathrm{c}}, \mathrm{T}_{\mathrm{y}}\right\}_{\text {field }}
$$

with the transformation $(\alpha=\mathrm{u}, \mathrm{c}, \mathrm{l})$

$$
\mathrm{T}_{\alpha}=c_{\alpha, x} \mathrm{~T}_{x}+c_{\alpha, y} \mathrm{~T}_{\mathrm{y}}+c_{\alpha, z} \mathrm{~T}_{z}
$$

If the energetic separation of the triplet substates is larger than their kinetic widths, their decay may be treated separately, and the ISC rate constants of the coupled states (31) are given by

$$
k_{0, \alpha}=\left|c_{\alpha, x}\right|^{2} k_{0, x}
$$

Without any relaxation among the triplet substates the yield $Y_{p}$ of products in the overall decay is

$$
Y_{\mathrm{p}}=\frac{1}{3} \sum_{\alpha} \frac{k_{\mathrm{p}}}{k_{\mathrm{p}}+k_{0, \alpha}}
$$

Here, again, we find the dilution principle described in the last section. The extreme results are obtained for $Y_{\mathrm{p}}$ either in the case of complete localization of $\mathrm{T}_{x}\left(c_{\alpha, x}\right.$ $=1,0,0)$ or in the case of maximum delocalization $\left(c_{\alpha, x}\right.$ $=1 / 3$ for all $\alpha$ ). In the latter case the yield $Y_{p}$ is at a minimum and the average lifetime of the triplet is shortest.

Proceeding from the fixed-molecule situation to tumbling molecules in low-viscosity solvents brings with it the problem of different frames of reference for $H_{\mathrm{ZFS}}$ and $H_{\mathrm{so}}$ on the one hand and $H_{\mathrm{Z}}$ on the other. In this situation the mechanism is best visualized if one continues to consider the problem in the molecule-fixed frame. ${ }^{80}$ Then $H_{\mathrm{Z}}\left(B_{0}\right)$ becomes a randomly modulated interaction with zero average, and $\mathrm{T}_{x}, \mathrm{~T}_{y}$, and $\mathrm{T}_{z}$ remain the eigenstates of $H_{0}$ whatever the strength of the external magnetic field may be. We may then describe the influence of molecular tumbling and external magnetic field as inducing relaxation among the triplet substates $\mathrm{T}_{x}, \mathrm{~T}_{y}$, and $\mathrm{T}_{z}$ defined in the molecular frame. To represent this view schematically, we have supplemented scheme b in Figure 7 by relaxational transitions among these substates. Thus $\mathrm{T}_{y}$ and $\mathrm{T}_{z}$ become connected to $T_{x}$ and may share its propensity for the intersystem crossing process. Consequently the chemical yield $Y_{p}$ will become a function of the effective relaxation rate constant among the triplet substates. In order to observe a MFE it is required that in zero field the spin equilibrium among the substates is not attained, which means that sublevel-selective ISC must be of comparable rate or faster than molecular tumbling, which is the basic mechanism of relaxation among the spin substates. This rather stringent condition requires that in low-viscosity solutions ISC from the triplet should occur in a time of $\leq 0.1 \mathrm{~ns}$.

In a magnetic field the effective relaxation rate among the molecule-fixed substates will increase and so will the averaged ISC rate. Thus the chemical yield decreases in a magnetic field. The effect saturates at high fields, $B_{1 / 2}$ depending on the larger of either the sublevel-selective ISC rate, the molecular tumbling rate, or the ZFS (for examples of MFD curves, cf. section IV.C.3).

\section{E. Paramagnetic Conversion of Ortho and Para Hydrogen}

Ortho and para hydrogen are two "isomers" of molecular hydrogen differing in the alignment of their nuclear spins. In ortho hydrogen the nuclear spins are combined to a nuclear spin triplet, whereas the para form corresponds to a nuclear spin singlet combination. Both forms may be distinguished, e.g., by their different heat capacity, causing a difference in thermal conductivity. This difference results from the fact that the complete nuclear wave function (spin and spatial part) must be antisymmetric under the exchange of two protons because they are Fermi particles. Since the spin part is even for the ortho state, the rotational part must be odd $(L=1,3,5, \ldots)$ whereas in the para state, the 
spin function is odd and the rotational part of the wave function must be even $(L=0,2,4, \ldots)$. Thus the lowest rotational states of the two forms are separated by one rotational quantum of about $100 \mathrm{~cm}^{-1}$. At cryogenic temperatures the para form can be enriched on a catalytic surface. In the absence of catalytic materials establishment of the ortho/para equilibrium is quite slow, even at elevated temperatures. Catalytic conversion may be effected by two mechanisms. In the dissociative mechanism $\mathrm{H}_{2}$ molecules are dissociated due to the catalytic activity and recombine at random. This mechanism will not be of interest in this review. The other mechanism is based on the interaction of hydrogen molecules with paramagnetic centers either in the gas phase, in liquid solution, or on solid surfaces.

Except for a recent paper by Sugano and Ilisca ${ }^{81}$ (cf. section V.F), it has been assumed that the basic interaction responsible for the para/ortho conversion is the inhomogeneous dipolar magnetic field due to the paramagnetic spin, which is experienced with different strength and direction by the two nuclei in a hydrogen molecule. Therefore they will undergo different precessional motions and eventually change their relative spin alignment.

In this respect the mechanism is quite analogous to the change of electron spin in a radical pair, which is also due to different local magnetic fields at the sites of the two electron spins. Essential differences, however, lie in the fact that in radical pairs it is a constant intramolecular hyperfine coupling that governs the different motions of the two electron spins, whereas the two nuclear spins in the $\mathrm{H}_{2}$ molecule are reoriented due to an intermolecular anisotropic hyperfine interaction with a colliding paramagnetic particle. Another fundamental difference is that the different spin states of a radical pair are degenerate within the width of the coupling interaction, whereas in the ortho/para hydrogen case the energy difference is on the order of 100 $\mathrm{cm}^{-1}$. This requires energy exchange with other degrees of freedom during a collision with the paramagnetic particle. The latter point is essential and was first taken into account by Wigner. ${ }^{82}$

External MFEs on catalytic ortho/para hydrogen conversions have so far been only observed on magnetic surfaces (so-called magnetocatalytic effect; $\mathrm{cf}$. the review by Selwood ${ }^{26}$ ). Although the mechanisms of these processes are not yet fully understood, it seems to be clear that the mechanism is of the indirect type. The initial state manifold $\left\{i_{k}\right\}$ has to be constructed from the combined states of para hydrogen rotational states and the electron spin of the paramagnetic centers:

$$
\left\{i_{k}\right\}=\left\{p-\mathrm{H}_{2}(L=0,2, \ldots)\right\} \times\left\{\mathrm{S}_{\text {paramagn }}\right\}
$$

The interaction with the ortho hydrogen manifold occurs by the mechanism mentioned above. The interaction operator $H^{\prime}$ is randomly modulated according to the statistics of the collisions. The corresponding theory would be of the Ib/IIa type. As has been pointed out by Ilisca ${ }^{83}$ (cf. section V), one must assume that large deviations from equilibrium are generated in the $\left\{i_{k}\right\}$ manifold due to the adsorption/desorption kinetics of $\mathrm{H}_{2}$ molecules on the surface. It is mainly the magnetic effect on this polarization that is responsible for the kinetic effects of the magnetic field on the overall para/ortho conversion rate.
TABLE 3. Acronyms in Use for Various Types and Detection Modes of Magnetokinetic Effects

\begin{tabular}{|c|c|c|}
\hline acronym & meaning & $\mathrm{ref}^{a}$ \\
\hline ADMR & absorbance-detected magnetic resonance & 88 \\
\hline CIDEP & $\begin{array}{l}\text { chemically induced dynamic electron } \\
\text { polarization }\end{array}$ & 84,85 \\
\hline CIDNP & $\begin{array}{l}\text { chemically induced dynamic nuclear } \\
\text { polarization }\end{array}$ & 86,87 \\
\hline CIDNP·NR & $\begin{array}{l}\text { CIDNP-detected nuclear magnetic } \\
\text { resonance }\end{array}$ & 89,110 \\
\hline DF-ODMR & delayed-fluorescence ODMR & 90 \\
\hline FDMR & fluorescence-detected magnetic resonance & 91,92 \\
\hline MARY & $\begin{array}{l}\text { magnetic field modulation of reaction } \\
\text { yields }\end{array}$ & 93 \\
\hline MIE & magnetic isotope effect & $94-96$ \\
\hline MODS & $\begin{array}{l}\text { magnetooptical absorbance difference } \\
\text { spectroscopy }\end{array}$ & 97 \\
\hline MODSC & $\begin{array}{l}\text { magnetooptically detected spin } \\
\text { conversion spectroscopy }\end{array}$ & 98,309 \\
\hline ODESR & optically detected electron spin resonance & 99 \\
\hline ODMR & optically detected magnetic resonance & $100-105$ \\
\hline RYDMR & $\begin{array}{l}\text { reaction-yield-detected magnetic } \\
\text { resonance }\end{array}$ & 106 \\
\hline RYDMAR & $\begin{array}{l}\text { reaction-yield-detected magnetic } \\
\text { resonance }\end{array}$ & 107 \\
\hline SPDL & spin-dependent luminescence & 108 \\
\hline TDR & triplet-doublet resonance & 109 \\
\hline TTR & triplet-triplet resonance & 109 \\
\hline
\end{tabular}

${ }^{\text {a }}$ References denote first users of these acronyms or pioneers in developing the respective mechanisms and experimental techniques.

\section{Experimental Methods}

The various mechanisms described in the last section are detectable in rates and yields of particular chemical reactions or related physical processes. A great variety of experimental methods have been applied to induce and detect such changes. In connection with these a number of acronyms have been created that have been more or less accepted in the literature. These are collected in Table 3. Their number may give an exaggerated impression of diversification in the field. It should be noted, however, that different terms are in use for rather similar methods.

Four main groups of techniques may be distinguished:

(i) Chemical reactions conducted in stationary magnetic fields may produce spin-polarized products. Spin polarization of nuclei (CIDNP) or radical electrons (CIDEP) is detected by magnetic resonance during or immediately after the reaction. A particular way of detecting chemically induced spin polarization might even utilize the generation of radio-frequency maser activity. ${ }^{111}$ Chemically induced magnetic polarization is an enormous field of its own and will not be covered in this review. However, selected references will be made to CIDMP work (this term includes both nuclear and electron spin polarization), which is of interest in relation to our topic.

(ii) The overall change of yields and kinetics of chemical and related physical processes due to the effect of a static magnetic field may be directly detected. The magnetic field dependence of such effects has been referred to as a MARY "spectrum", ${ }^{93}$ particularly when discussed in relation with RYDMR spectra (cf. below). The MARY acronym is, however, not generally used for magnetic-field-dependent reaction yield effects. Various physical or chemical parameters, to be surveyed 
below, have been used to monitor the MFD of reaction yields. Some of these modes of detection have been assigned special names such as MODSC ${ }^{98,309}$ for both luminescence and absorption detection, MODS $^{97}$ for modulated absorption spectroscopy, and TTR or $\mathrm{TDR}^{109}$ for photoconductivity detection in molecular crystals (cf. Table 3 for the meaning of these acronyms).

(iii) Different nuclear magnetic moments of different isotopes may cause differences in reaction rates and yields, which may eventually lead to a selective distribution of different isotopes over the products of a reaction. These effects constitute the field of magnetic isotope effects. ${ }^{94-96}$

(iv) The effect of a static magnetic field on reaction kinetics and yields may be modified by the absorption of resonant microwave radiation, offering the possibility of measuring the magnetic resonance spectra of particular reaction intermediates responsible for the MFE. This method is called reaction-yield-detected magnetic resonance (RYDMR). ${ }^{106}$ Many subcases are distinguished by special terms. Especially for luminescence detection (ODMR) a number of equivalent terms are in use (FDMR, ODESR, SPDL; cf. Table 3). The absorption method analogue to ODMR is ADMR. ${ }^{88}$ The method of CIDNP-NR ${ }^{89,110}$ is also a RYDMR subcase, since it uses resonant radiofrequency fields during a reaction, the effect of which is detected via CIDNP in the diamagnetic products.

In the following we provide a short survey of the various methods used so far to monitor magnetokinetic effects and give some basic information on magnetic equipment applied in these investigations.

\section{Magnets and Microwave Equipment}

Magnetic fields up to $0.1 \mathrm{~T}(1000 \mathrm{G})$ may be conveniently produced by a pair of Helmholtz coils. These have the advantage that they are inexpensive, the sample space is easily accessible from all sides, and, furthermore, the magnetic field may be rapidly modulated to allow a more sensitive detection of the effects (for applications cf., e.g., ref 112 and 113).

Permanent magnets, which can provide fields up to 1.5 $\mathrm{T}$, are practically not in use since their field is not variable. In this context it may be of interest, however, that microscopic ferromagnetic particles have been used for magnetic modulation of chemical reaction yields. ${ }^{114}$

For magnetic fields up to $4 \mathrm{~T}$, electromagnets are convenient. ${ }^{115}$ Smaller types of about $50-\mathrm{kg}$ weight usually can provide magnetic fields up to $1 \mathrm{~T}$ between flat poles of 1-2-cm gap. With tapered poles and gaps of $0.5-1 \mathrm{~cm}$, field strengths up to $2 \mathrm{~T}$ are attainable. Electromagnets of several hundred kilogram weight are necessary for magnetic fields of about $2.5 \mathrm{~T}$ with $2-3-\mathrm{cm}$ gaps and up to $4 \mathrm{~T}$ with about $0.5-\mathrm{cm}$ pole gaps.

Pulsed fields up to $7 \mathrm{~T}$, which are approximately constant over a period of about $3 \mathrm{~ms}$, have been produced by discharging a capacitor through a solenoid. .16,772,773 $^{2}$

The field region up to $15 \mathrm{~T}$ is accessible by use of superconducting magnets, ${ }^{117}$ whereas ultrahigh fields up to $25 \mathrm{~T}$ are realized by disk coils, so-called Bitter magnets, ${ }^{118}$ with an electrical power consumption of several megawatts, necessitating very efficient water cooling. Instruments of this type are usually available only in special high-field laboratories, e.g., the Francis
Bitter National Magnetic Laboratory of MIT ${ }^{119}$ or the High-Field Magnetic Laboratory of Grenoble. ${ }^{120}$ Thus it is understandable that not many investigations of chemical reactions in ultrahigh magnetic fields have been reported.

The RYDMR techniques require that a considerable fraction of resonant spin flips take place during the geminate periods of paramagnetic particle pairs, which are typically in the submicrosecond time regime. Thus the $B_{1}$ field must be on the order of $1 \mathrm{G}$ or larger, meaning that much higher microwave powers are necessary than in conventional ESR spectroscopy. Otherwise RYDMR spectrometers are very similar to ESR spectrometers, however without using the absorbed microwave power for monitoring the resonance. For the usual X-band technique $(9.5 \mathrm{GHz})$ normal ESR cavities with facilities for optical detection are in use. The required microwave power is produced by a clystron valve or a Gunn diode combined with traveling-wave tube amplifiers. Representative examples of power specifications are $5-\mu \mathrm{s}$ pulses at $350 \mathrm{~W}^{121}$ or $1.6-\mu$ s pulses at $2000 \mathrm{~W} .{ }^{122}$ However, much shorter pulses of about $60 \mathrm{~ns}$, still with $B_{1} \approx 1.5 \mathrm{G}$, corresponding to a $\pi / 2$ pulse intensity, have been also realized in applications of time-resolved RYDMR in a spin-echo spectrometer. ${ }^{123,124}$

RYDMR has also been applied at low fields with frequencies in the $250-1700-\mathrm{MHz}$ region where powerful frequency generators of about $50 \mathrm{~W}$ are available. As in ESR, at these frequencies special cavities, e.g., a strip-line cavity ${ }^{125}$ or a split-ring resonator, ${ }^{107,126}$ have to be used.

\section{Chemical Analysis}

If MFEs are borne out in product yields of irreversible reactions, conventional methods of chemical analysis may be applied after the reaction has been terminated. Thus MFEs in gas-phase reactions have been analyzed by using gas chromatography, ${ }^{127}$ mass spectrometry, ${ }^{128}$ or a combination of both. ${ }^{129}$ Magnetic-field-dependent yields of reactions in solution have been analyzed by vapor-phase chromatography after suitable pretreatment of the reaction mixtures. ${ }^{130-132}$ Application of thin-layer chromatography ${ }^{133-135}$ and of high-pressure liquid chromatography ${ }^{136}$ has also been reported in relation with the detection of magnetic field effects.

In favorable cases the reaction yield may be obtained from an in situ analysis of the reaction mixture, as has been applied, e.g., by Molin and co-workers, ${ }^{137,138}$ who used ${ }^{1} \mathrm{H}$ NMR and ${ }^{19} \mathrm{~F}$ NMR to detect magnetic field effects on the yield of thermal radical reactions performed directly in a NMR sample tube. Even more favorable are cases where the reaction can be followed by continuous probing of some characteristic physical parameter (cf. section 4).

Conventional chemical analysis may be also applied to determine the MFD of the yield of short-lived transient species, if it is possible to trap these species in relatively stable products. Examples are the use of spin traps for radicals ${ }^{139}$ and of suitable scavengers of singlet oxygen. 140,141

Finally, the interesting case of a MFE on the molecular weight distribution obtained in emulsion polymerization must be mentioned. ${ }^{142}$ 


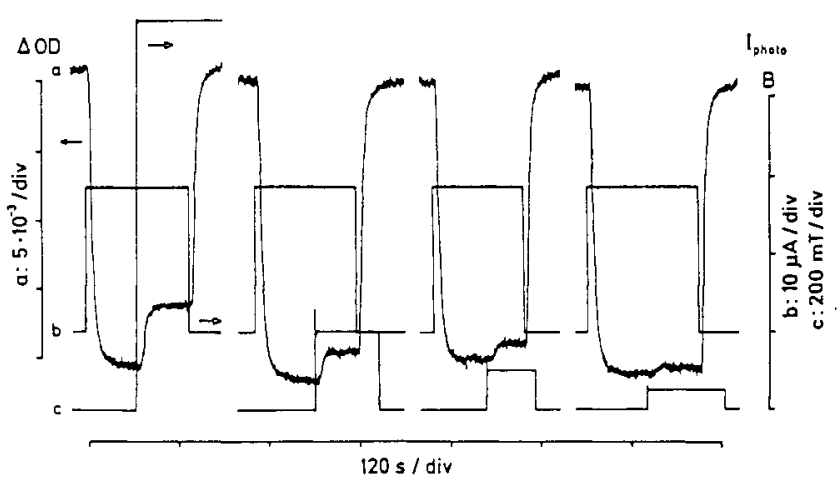

Figure 8. Detection of MFE by continuous-photolysis in a flow-through apparatus, for example the photoreduction of thionine (6S) by $p$-iodoaniline in methanol (from Schlenker and Steiner ${ }^{152}$ ). The diagram shows $Y / t$ traces of (a) the absorption signal at fixed wavelength, (b) photolytic light intensity, and (c) magnetic field strength.

\section{Magnetic Isotope Enrichments}

If kinetic MIEs occur in irreversible chemical reactions, this results in magnetic isotope enrichments. So far, reliable results have been reported for the magnetic isotopes ${ }^{13} \mathrm{C}$ and ${ }^{17} \mathrm{O}$ only. Enrichments of ${ }^{13} \mathrm{C}$ have been detected by ${ }^{13} \mathrm{C} N \mathrm{NMR}^{96}$ and from ${ }^{13} \mathrm{C}$ satellites in ${ }^{1} \mathrm{H}$ NMR spectra. ${ }^{143,146}$

Enrichments of ${ }^{17} \mathrm{O}$ have been detected from mass spectroscopic data of oxygen developed in endoperoxide thermolysis ${ }^{147}$ or from IR data based on the different carbonyl frequencies of $\mathrm{R}_{2} \mathrm{C}^{16} \mathrm{O}\left(1717 \mathrm{~cm}^{-1}\right)$ and $\mathrm{R}_{2} \mathrm{C}^{17} \mathrm{O}$ $\left(1705 \mathrm{~cm}^{-1}\right) .{ }^{148}$

\section{Rates and Quantum Yields from Photostationary Kinetics}

In many cases the rates of photoreactions resulting in permanent product formation can be conveniently followed in continuous-illumination experiments. The usual method, as for the determination of photochemical quantum yields, is to follow the reaction by optical absorption spectroscopy, either by continuously recording at a fixed wavelength or by recording complete spectra at certain intervals of irradiation time. . $^{149-151}$

The reproducibility of photokinetic slopes in different runs is often a limiting factor in the accuracy to which MFEs can be determined. In order to get more direct information on the MFD of photochemical quantum yields, Schlenker and Steiner ${ }^{152}$ developed a special technique whereby the solution is irradiated in a flowthrough cuvette. Opening or blocking the irradiation light produces step-like changes in the absorption signal, which is continuously recorded at a fixed wavelength in a second flow-through cuvette positioned in a UV detector. The height of the steps is proportional to the quantum yield of the reaction. Switching on and off of a magnetic field at the irradiation cuvette during the irradiation periods produces corresponding steps in the absorption signal trace, from which the MFE on the quantum yield is directly obtained. In Figure 8 an example of a MFE detection by this technique is shown.

Other detection methods for continuously following magnetic-field-dependent reactions have made use of variations of pressure in gas mixtures ${ }^{130}$ or of thermal conductivity changes in cases of hydrogen evolution. ${ }^{153}$ The latter method is also generally used in order to follow the rate of magnetocatalytic ortho/para hydrogen conversion. ${ }^{154,155}$

In the case of reversible photoreactions, photostationary states are established under continuous illumination. Although the stationary concentrations of short-lived transients (such as triplets or radicals) may be quite small, modulation techniques may provide the required sensitivity to record absorption signals of these species and determine the MFD of their stationary concentrations. Thus, Bube et al. ${ }^{112}$ using two $\mathrm{CW}$ lasers for excitation and probing and applying squarewave modulation of the magnetic field together with phase-sensitive detection of absorption were able to measure the MFD of anthracene ion formation in the reaction of singlet excited anthracene with diethylaniline.

The MODS technique of Hoff et al. ${ }^{97}$ is based on the same principle.

\section{Flash Photolysis}

The application of photostationary modulation techniques for probing the absorption of radicals and electronically excited triplets is rather unusual. The standard method for determining the yields and kinetics of such intermediates is flash photolysis. The conventional flash technique, however, is rarely used for the investigation of magnetic field effects (cf., e.g., ref 156), probably because most conventional flash equipment uses a close coupling of cuvette and flash lamps, making it difficult to apply a magnetic field to the sample. This problem does not arise with laser flash excitation, where very small excited volumes can be probed even in magnets with difficult access to the sample space.

Whereas single-shot laser techniques usually provide only a rather qualitative picture of the MFD, the accuracy can be greatly improved by the use of signal averaging and time-selective absorption sampling. In the latter method two laser pulses are applied, where the first, strong pulse serves to excite the sample and the second, weaker pulse probes the transient absorption at a fixed delay time. ${ }^{157,158}$ This method has been perfected by Treichel et al., ${ }^{159}$ yielding an excellent reproducibility of the MFEs (cf. Figure 19).

The other efficient method to reduce the signal scatter, which is mainly due to a variation of singlepulse energies, is on-line processing of many repeated transient signals by the use of microcomputer facilities. Laser flash equipment working on this principle has been described by Ulrich et al. ${ }^{160}$ An interesting novel development has recently been reported by McLauchlan and co-workers. ${ }^{121}$ They use a series of pulses with the magnetic field on and off in alternation for successive laser pulses, so that by suitable data processing they achieve a real-time differencing of the signal intensity with respect to the magnetic field. Their spectrometer may be used for measuring the MFD of reaction yields as well as for recording RYDMR spectra.

So far, the time resolution of laser flash spectroscopy as applied to investigate MFEs has been restricted to the nanosecond time regime. This usually does not suffice to yield a good time resolution of the geminate recombination kinetics of radical pairs in low-viscosity solutions. Magnetic-field-dependent kinetics is, how- 
ever, particularly clearly borne out in micellar ${ }^{161,162}$ or microemulsion solution. ${ }^{163,164}$

\section{Luminescence}

Luminescence may be detected very sensitively and very accurately and is an ideal method for determining MFEs if electronically excited products are formed in the magnetic-field-dependent process. The simplest technique is to measure stationary luminescence intensities as a function of the magnetic field strength. Many examples of MFE detection using this method are provided in section IV for solid-state, liquid, and gas-phase systems and in flames.

Luminescence may be excited optically by high-energy radiation or may result from chemiluminescent or electrochemiluminescent reactions. In the case of small MFEs it has been advantageous to modulate the magnetic field and use phase-sensitive detection of luminescence. In this way Frankevich and co-workers ${ }^{113}$ were able to achieve a relative sensitivity of better than $5 \times 10^{-5}$, which allowed them to resolve the MFD of luminol chemiluminescence, showing a maximum effect of only $0.15 \%$ at high fields.

Time-resolved MFEs have been observed in radioluminescence applying the single-photon-counting technique. ${ }^{165-168}$ Particularly high reproducibility of the MFEs is obtained if the accumulation of single-photon statistics is made intermittently with periods of magnetic field on and off, so that long-time drifts cannot wipe out the MFE. ${ }^{169,761}$

Luminescence methods have also been applied to probe the yields of short-lived products formed in their ground states. The method used was a two-step laser-excited fluorescence detection, where the second (probing) laser pulse excites the product and the integral of the resulting fluorescence pulse is a measure of the concentration of this species. The kinetics of its formation and decay may be determined from a variable delay of the second laser pulse. ${ }^{170,171}$

\section{Photocurrents}

If a magnetic-field-sensitive reaction mechanism produces charge carriers in a medium of low self-conductivity, current measurements are an obvious method of investigation. So far little use has been made of such techniques in solutions. ${ }^{172,762,763}$ There are, however, more investigations applying photoconductivity measurements for detecting the MFD of processes occurring in the bulk (cf., e.g., ref 173 and 174) or at surfaces (cf., e.g., ref 175 and 176) of organic molecular crystals. Other examples may be cited from the field of inorganic semiconductors. ${ }^{177-179}$

\section{Experimental Studies}

\section{A. In the Gas Phase}

Magnetokinetic effects in the gas phase have been observed in photoluminescence, in light emission from flames, and in irreversible photochemical change. A survey of these is given in Tables 4 and 5. Most work has been done on the photoluminescence of small molecules, the most prominent cases of which $\left(\mathrm{I}_{2}, \mathrm{NO}_{2}\right.$,
$\mathrm{CS}_{2}$, glyoxal) have been also reviewed in some detail by Küttner et al. ${ }^{210}$ and by Lin and Fujimura. ${ }^{29}$ Many of the observations listed are due to magnetic field effects on radiationless decay and not accompanied by reversible or irreversible chemical change. Since they appear, however, as a phenomenological and mechanistical unity with truly chemical effects, we have included them, too, the more so as any process determining the lifetime of an electronically excited state must be reflected in the quantum yield of a competing photochemical reaction from this state.

A typical feature of MFEs in the gas phase is that they are usually found with small molecules, which are characterized by a sparse density of rovibrational states. Thus irreversible transition to the final state manifold often requires collisions to provide an effective continuum. Such a mechanism will become evident in the pressure dependence. Magnetic fields may influence either the pressure-dependent or -independent part of the decay rate. Other important mechanistic distinctions arise from the magnetic moment of the initial state. Thus paramagnetic triplet or doublet states may show magnetic field dependence even in multiplicityconserving processes $\left(\mathrm{I}_{2}, \mathrm{NO}_{2}\right)$ whereas the magnetic field sensitivity of excited singlet states is usually related to intersystem crossing (ISC) processes (glyoxal).

\section{Photoluminescence Studies}

The first case of magnetic fluorescence quenching was reported by Steubing ${ }^{180}$ in 1913 and further corroborated by a number of later investigations. ${ }^{181-183}$ The magnetic quenching effect in iodine is due to a magnetic-field-induced predissociation of the ${ }^{3} \Pi_{0^{+} u}$ state (note that the "fluorescence" is a ${ }^{3} \Pi_{0^{+}} \rightarrow X^{1} \Sigma^{+}$transition), which was first suggested by Franck and Grotrian ${ }^{184}$ and substantiated by experiments of Turner. ${ }^{185}$ According to van Vleck ${ }^{223}$ the magnetically induced transition leads to a nonbonding ${ }^{3} \Pi_{0^{+} u}$ state, which is forbidden in the absence of a magnetic field (cf. section V). Investigating details of the excitation wavelength and magnetic field dependence, Degenkolb et al. ${ }^{186}$ and Chapman and Bunker ${ }^{187}$ were able to construct the potential curve of the dissociative state by evaluating the Franck-Condon factors for magnetic predissociation of the various vibrational levels of the ${ }^{3} \Pi_{0^{+} u}$ state. According to a suggestion by Vigue et al., ${ }^{190}$ the perturbing state should be a $1 \mathrm{u}$ state, to which also a rotational predissociation in the absence of a magnetic field can occur. That this is in fact the case was corroborated by the detection of a magnetic-field-dependent weak circular polarization of the emitted iodine fluorescence.

Another case of magnetic-field-induced radiative dissociation of electronically excited $\mathrm{Hg}_{2}$ reported by Franck and Grotian ${ }^{184}$ was not confirmed in later experiments. 191

Proceeding from iodine to the triatomic examples $\mathrm{NO}_{2}, \mathrm{SO}_{2}$, and $\mathrm{CS}_{2}$, the conditions and mechanistic details of the magnetic effects become more complex. In $\mathrm{NO}_{2}$, which was investigated by Butler et al., ${ }^{192-194}$ a magnetic field enhances the collision-induced fluorescence quenching. The magnetic field dependence of the fluorescence yield (cf. Figure 9) may be described by a Lorentzian line shape centered at $B_{0}=0$. However, the half-field values and the modulation depth of 
TABLE 4. Magnetic Luminescence Quenching in the Gas Phase

\begin{tabular}{|c|c|c|c|}
\hline \multirow[t]{2}{*}{ emitting molecule } & \multicolumn{2}{|r|}{ observations and objectives } & \multirow[t]{2}{*}{ ref } \\
\hline & & A. Photoluminescence & \\
\hline \multirow[t]{6}{*}{$\mathrm{I}_{2}$} & \multicolumn{2}{|c|}{ magnetic fluorescence quenching } & $\begin{array}{l}\text { Steubing, }{ }^{180,181} \text { Wood and Ribaud, } \\
\text { Oldenburg }\end{array}$ \\
\hline & & Franck and Grotrian ${ }^{184}$ \\
\hline & \multirow{2}{*}{\multicolumn{2}{|c|}{$\begin{array}{l}\lambda_{\text {exc }} \text { dependence of magnetic quenching } \\
\lambda_{\text {erc }}, p, T \text {, and } B_{0} \text { (up to } 7 \mathrm{~T} \text { ) dependence of fluorescence } \\
\text { magnetic auenching of single rovibronic levels, FC factors }\end{array}$}} & Turner ${ }^{185}$ \\
\hline & & & Degenkolb et al. ${ }^{186}$ \\
\hline & \multirow{2}{*}{\multicolumn{2}{|c|}{$\begin{array}{l}\text { magnetic quenching of single rovibronic levels, FC factors } \\
\text { circular polarization due to magnetic quenching }\end{array}$}} & Chapman and Bunker ${ }^{187}$ \\
\hline & & & Broyer et al., ${ }^{188}$ Vigue et al. ${ }^{189,190}$ \\
\hline $\mathrm{Hg}_{2}$ & \multirow{2}{*}{\multicolumn{2}{|c|}{$\begin{array}{l}\text { suggestion of magnetic luminescence induction by reaction } \mathrm{Hg}_{2} * \rightarrow 2 \mathrm{Hg}+h \nu \\
\text { effect in ref } 183 \text { probably a magnetochemical artifact }\end{array}$}} & Franck and Grotrian ${ }^{183}$ \\
\hline $\mathrm{NO}_{2}$ & & & $\begin{array}{l}\text { Niewodniczansky } \\
\text { Butler et al } 192-194\end{array}$ \\
\hline & \multicolumn{2}{|c|}{$\begin{array}{l}\text { MFD of collision-induced fluorescence quenching, erratic } \lambda_{\text {exc }} \text { dependence, } \\
\text { saturation at } B_{0}>1 \mathrm{~T}\end{array}$} & \\
\hline \multirow{2}{*}{$\mathrm{SO}_{2}$} & \multirow{2}{*}{\multicolumn{2}{|c|}{$\begin{array}{l}\text { magnetic quenching of fluorescence and phosphorescence } \\
p \text { and } B_{0} \text { dependence of fluorescence and phosphorescence lifetime }\end{array}$}} & Makarov et al., ${ }^{195}$ Sorokin et al. ${ }^{128}$ \\
\hline & & & Makarov et al. ${ }^{196}$ \\
\hline \multirow{3}{*}{$\mathrm{CS}_{2}$} & & ing of $\mathrm{N}_{2}$-laser-excited ${ }^{1} \mathrm{~A}_{2}$ and ${ }^{1} \mathrm{~B}_{2}$ excited states, no & Nagakura et al. ${ }^{197-200}$ \\
\hline & \multicolumn{2}{|c|}{$\begin{array}{l}\text { MFE on }{ }^{1} \mathrm{~A}_{\mathrm{i}} \text {-resonance fluorescence suggested to be due to Zeeman detuning of } \\
\text { absorption; collision-induced magnetic quenching of fluorescence continuum } \\
\text { confirmed }\end{array}$} & Silvers et al. ${ }^{201}$ \\
\hline & \multicolumn{2}{|c|}{$\begin{array}{l}\text { magnetic fluorescence quenching of banded }\left({ }^{1} \mathrm{~A}_{2}\right) \text { emission: } \\
\text { picosecond-time-resolved detection and highly dispersed excitation spectrum } \\
\text { with supersonic jet }\end{array}$} & Imamura et al. ${ }^{225}$ \\
\hline \multirow{2}{*}{$\begin{array}{l}\mathrm{H}_{2} \mathrm{CO} \text { and } \mathrm{D}_{2} \mathrm{CO} \\
\mathrm{D}_{2} \mathrm{CO}\end{array}$} & \multirow{2}{*}{\multicolumn{2}{|c|}{$\begin{array}{l}\text { oscillatory MFD of collision-induced fluorescence quenching } \\
\text { collision-induced magnetic quenching of } 4^{0} \text { and } 4^{1} \text { vibrational levels of }{ }^{1} \mathrm{~A}_{2} \text { state } \\
\text { time-resolved measurements }\end{array}$}} & Sorokin et al. $128,202,203$ \\
\hline & & & $\begin{array}{l}\text { Orita et al. } \\
\text { Orita et al. }\end{array}$ \\
\hline \multirow[t]{6}{*}{ glyoxal } & \multicolumn{2}{|c|}{ low-field magnetic fluorescence quenching } & Dong and Kroll ${ }^{206}$ \\
\hline & \multicolumn{2}{|c|}{$\begin{array}{l}\text { magnetic quenching of fluorescence, enhancement of phosphorescence, } \\
\text { saturation at } B_{0}=1 \mathrm{~T}\end{array}$} & Matsuzaki and Nagakura ${ }^{207-209}$ \\
\hline & \multirow{2}{*}{\multicolumn{2}{|c|}{$\begin{array}{l}\text { evidence of collision-induced magnetic ISC, independence of quencher } \\
\text { excitation wavelength dependence of magnetic fluorescence quenching }\end{array}$}} & Küttner et al. $24,210,211$ \\
\hline & & & Nakamura et al. ${ }^{212}$ \\
\hline & \multirow{2}{*}{\multicolumn{2}{|c|}{$\begin{array}{l}\text { high-field ( } 0-10 \mathrm{~T}) \mathrm{S}-\mathrm{T} \text { level anticrossing spectroscopy } \\
\text { magnetic quenching of single rovibronic levels }\end{array}$}} & Lombardi et al. ${ }^{120}$ \\
\hline & & & Michel and Tric ${ }^{72}$ \\
\hline glyoxal- $d_{2}$ & \multicolumn{2}{|c|}{$\begin{array}{l}\text { collision-induced magnetic fluorescence quenching, independence of quencher } \\
\text { and deuteriation }\end{array}$} & Küttner et al. ${ }^{24,211}$ \\
\hline methylglyoxal & \multirow{2}{*}{\multicolumn{2}{|c|}{$\begin{array}{l}\text { complex MFD of decay kinetics } \\
\text { MFD beats in single rotational level decay in supersonic beam }\end{array}$}} & Hashimoto et al. ${ }^{213}$ \\
\hline biacetyl & & & Henke et al. ${ }^{214}$ \\
\hline pyrimidine & \multicolumn{2}{|c|}{$\begin{array}{l}\text { MFD beats in fluorescence polarization after single rovibrational level } \\
\text { excitation in supersonic beam }\end{array}$} & Ohta et al. ${ }^{226}$ \\
\hline & \multirow{2}{*}{\multicolumn{2}{|c|}{$\begin{array}{l}\text { B. Emission from Flames and Microwave Discharges } \\
\text { magnetic enhancement of } \mathrm{D} \text {-line emission from } \mathrm{Na} \text { salts in } \mathrm{H}_{2} / \mathrm{O}_{2} \text { flames } \\
\text { highly dispersed observation of emission from salt aerosols in flames: MFE is } \\
\text { due to reduction of line reversion by Zeeman effect }\end{array}$}} & Wakayama et al. ${ }^{215}$ \\
\hline $\mathrm{Na}, \mathrm{Cs}$ & & & Sorokin et al. \\
\hline $\begin{array}{ll}\mathrm{OH} \\
\mathrm{HPO}\end{array}$ & \multirow{2}{*}{\multicolumn{2}{|c|}{$\begin{array}{l}\text { magnetic enhancement of } \mathrm{A}^{2} \Sigma^{+} \rightarrow \mathrm{X}^{2} \Pi \text { emission from } \mathrm{H}_{2}\left(\text { or } \mathrm{C}_{3} \mathrm{H}_{8}\right) / \mathrm{O}_{2} \text { flames } \\
\text { magnetic quenching of } \mathrm{A}^{1} \mathrm{~A}^{\prime \prime} \rightarrow \mathrm{X}^{1} \mathrm{~A}^{\prime \prime} \text { emission from }\left(\mathrm{NH}_{4}\right)_{2} \mathrm{HPO}_{4} \text { in } \mathrm{H}_{2} / \mathrm{O}_{2} \\
\text { flames }\end{array}$}} & Hayashi ${ }^{216,217}$ \\
\hline HPO & & & Wakayama et al. ${ }^{218}$ \\
\hline $\begin{array}{l}\text { SnH } \\
\text { NO }\end{array}$ & $\begin{array}{l}\text { magnetic quenchin } \\
\text { magnetic quenchin } \\
\quad \operatorname{NO}\left(B^{2} \Pi_{r}\right)\end{array}$ & $\begin{array}{l}{ }^{4} \Sigma \rightarrow \mathrm{X}^{2} \Pi_{1 / 2} \text { emission from } \mathrm{SnCl}_{2} \text { in } \mathrm{H}_{2} / \mathrm{O}_{2} \text { flames } \\
\text { f microwave discharge afterglow from } \mathrm{N}\left({ }^{4} \mathrm{~S}\right)+\mathrm{O}\left({ }^{3} \mathrm{P}\right) \rightarrow\end{array}$ & Fukuda et al. ${ }^{219}$ \\
\hline & & & 保 \\
\hline & eaction & effect & ref \\
\hline $\begin{array}{l}\text { cis-2-butene } \\
\mathrm{SO}_{2}+\mathrm{C}_{5} \mathrm{H}_{12} \\
\mathrm{D}_{2} \mathrm{CO}-h_{\nu} \rightarrow \\
\mathrm{OHC}-\mathrm{CHO}- \\
\text { cycloheptane }\end{array}$ & $\begin{array}{l}\rightarrow \text { trans-2-butene } e^{a} \\
-h \nu \rightarrow \mathrm{HSO}_{2} \mathrm{C}_{5} \mathrm{H}_{11} \\
\mathrm{D}_{2}+\mathrm{CO} \\
h \nu \rightarrow \mathrm{H}_{2} \mathrm{CO}+\mathrm{CO} \\
-h \nu \rightarrow 6 \text {-heptenal }\end{array}$ & $\begin{array}{l}\text { increase of rate by } 30 \% \text { in magnetic field of } 8.5 \mathrm{~T} \\
\text { MFE on quantum yield }-30 \% \text { for } B_{0}>0.2 \mathrm{~T} \\
\text { MFE on quantum yield }+6 \% \text { at } 0.4 \mathrm{~T}, 0 \% \text { at } 0.8 \mathrm{~T} \\
\text { MFE on quantum yield }-15 \% \text { at } 0.3 \mathrm{~T} \text { and } p=0.05 \text { Torr } \\
\text { magnetic decrease of quantum yield }-3.6 \% \text { at } 0.6 \mathrm{~T} \\
\text { CIDNP in the gas phase }\end{array}$ & $\begin{array}{l}\text { Falconer and Wasserman }{ }^{127} \\
\text { Sorokin et al. }{ }^{128} \\
\text { Sorokin et al. }{ }^{128} \\
\text { Sorokin et al }{ }^{220} \\
\text { Stich et al. }{ }^{129} \\
\text { Dushkin et al. }{ }^{221}\end{array}$ \\
\hline $\mathrm{Cs}_{2}\left({ }^{1} \Pi_{u}\right) \rightarrow 2$ & $\mathrm{Cs}\left({ }^{2} \mathrm{P}_{3 / 2}\right)$ & $\begin{array}{l}\text { predissociation leads to selective magnetic substate } \\
\text { populations of } \mathrm{Cs} \text { atoms }\end{array}$ & Kato and Onomichi ${ }^{222}$ \\
\hline "Sensitization & $\mathrm{y} \mathrm{I}_{2} * \rightarrow 2 \mathrm{I}$ & & \\
\hline
\end{tabular}

the fluorescence yield by the magnetic field are of a rather erratic wavelength dependence. It is assumed that the magnetic field couples the fluorescing doublet state to some intermediate state that is itself coupled to a continuum via the collisions. The nature of the intermediate state is not clear.

In $\mathrm{SO}_{2}$ fluorescence and phosphorescence have been observed to be magnetically quenched..$^{128,195}$ The fact that the phosphorescence lifetime is magnetic field independent ${ }^{196}$ indicates that fluorescence quenching must go along with a reduction of the intersystem crossing efficiency. The fluorescence lifetime shows an interesting pressure-dependent magnetic field effect. ${ }^{196}$ At low pressures the lifetime increases by a magnetic field, which has been explained by a magnetically induced Douglas effect. The magnetic field is assumed to mix the electronically excited $B^{1} B_{1}$ state with rovibronic levels of the $\mathrm{X}^{1} \mathrm{~A}_{1}$ ground state. Whereas at low pressures this mixing just dilutes the oscillator strength of the electronically excited state, at higher pressures 


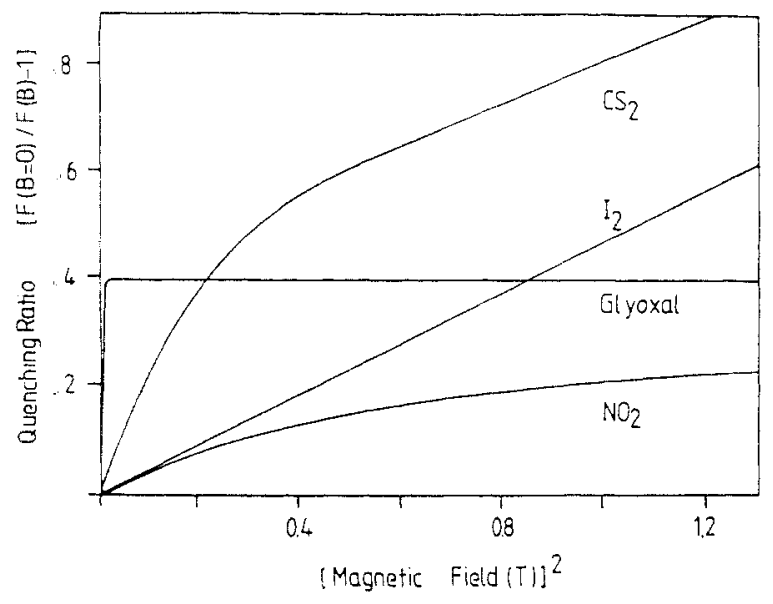

Figure 9. Types of MFD curves observed in magnetic quenching of photoluminescence in various gases (adapted from Küttner et al. ${ }^{24}$ ).

the collisional relaxation rate becomes higher than the intrinsic decay rate, and a magnetic shortening of the decay time of the excited state is observed.

In $\mathrm{CS}_{2}$ magnetic effects on the fluorescence from the ${ }^{1} \mathrm{~A}_{2}$ state and from the ${ }^{1} \mathrm{~B}_{2}$ state have been investigated by Nagakura et al. ${ }^{197-200}$ (cf. Figure 9 for the magnetic field dependence). The banded fluorescence from ${ }^{1} \mathrm{~A}_{2}$, which has also been investigated by Silvers et al., ${ }^{201}$ rather strongly decreases on application of a magnetic field $(-75 \%$ at $1.8 \mathrm{~T}){ }^{201}$ This effect is not borne out in the fluorescence lifetime in the nanosecond region, ${ }^{200}$ as originally reported, ${ }^{197}$ but only in the fluorescence intensity. Silvers et al. ${ }^{201}$ suggested that this effect is due to a magnetic broadening of the absorption lines, which leads to less efficient absorption of the narrow $\mathrm{N}_{2}$ laser line used in these investigations. Recently, the problem was reinvestigated by Nagakura and co-work$\mathrm{ers}^{225}$ using narrow-band excitation in a supersonic jet. The highly dispersed fluorescence excitation spectra clearly display the MFE on the fluorescence intensity, excluding an explanation based on Zeeman detuning of the absorption lines. Furthermore, picosecond timeresolved measurements of fluorescence decay revealed that the contribution of a fast-decaying component $\left(\tau_{\mathrm{f}}\right.$ $\approx 250 \mathrm{ps}$ ) increases grossly in a magnetic field, whereas the initial fluorescence intensity in the picosecond time domain is magnetic field independent.

The continuous emission from higher excited ${ }^{1} \mathrm{~A}_{2}$ levels shows a moderate collision-induced magnetic fluorescence quenching. ${ }^{201}$ For the ${ }^{1} \mathrm{~B}_{2}$ emission, showing biexponential decay kinetics, a magnetic field enhancement of the fast decay component was reported. 200

In the class of four-atomic molecules only formaldehyde $\left(d_{2}\right.$ and $\left.h_{2}\right)$ has been reported to show a magnetic-field-dependent fluorescence. ${ }^{128,202-205}$ Fluorescence from the ${ }^{1} \mathrm{~A}_{2}$ vibrational levels $4^{0}$ and $4^{1}$ in $\mathrm{D}_{2} \mathrm{CO}$ shows a decrease in lifetime in a magnetic field. From the pressure dependence it follows that this effect is collision induced. ${ }^{204}$ The effect has been analyzed in terms of contributions of various vibrational levels.

The magnetic fluorescence quenching of glyoxal, a molecule belonging to the intermediate level density case of radiationless transition theory, has found particular attention. It was first detected by Dong and $\mathrm{Kroll}^{206}$ and, independently, by Matsuzaki and Nagakura, ${ }^{207}$ who observed that the magnetic fluorescence quenching is accompanied by an increase in phosphorescence intensity, providing direct evidence that a magnetic-field-assisted intersystem crossing process is responsible for the fluorescence quenching. From the detailed pressure dependence of the effect, Schlag and co-workers ${ }^{24,210,211}$ concluded that the magnetic-fieldinduced intersystem crossing occurs only in collisions. The behavior of deuteriated glyoxal is similar. ${ }^{24,211}$ The effect was investigated with various quenching gases. Although the Stern-Volmer constants varied, the magnetic field effect on these was independent of the quencher species. The magnetic field effect saturates at very low fields as compared with other gas-phase cases (Figure 9). Special information on the singlettriplet coupling conditions in glyoxal has been obtained by Tramer and co-workers, ${ }^{120}$ who observed singlettriplet level anticrossing resonances in single-level resolved fluorescence and phosphorescence when applying very strong magnetic fields up to $10 \mathrm{~T}$ under collisionfree conditions. From these experiments it was deduced that the singlet-triplet coupling matrix elements are on the order of $10-100 \mathrm{MHz}$ and the level density in the triplet manifold is about 1 level per wavenumber. From another experimental investigation by Michel and Tric ${ }^{72}$ observing the magnetic field dependence of single rotational level fluorescence of glyoxal a quantitative model for the magnetic quenching of glyoxal fluorescence could be developed. In principle, it is based on a magnetic mixing of the final-state triplet sublevels, in combination with a nonlinear dependence of the collision-induced ISC rate constant on the singlettriplet mixing coefficients (for details on this and other models, cf. section V). Magnetic field effects on the fluorescence decay have also been found for methylglyoxal ${ }^{213}$ and biacetyl. ${ }^{214}$

\section{Light Emission from Flames or Microwave Discharges}

In flames luminescing excited states often originate as products of a generally not exactly known series of chemical reaction steps supplying the required excitation energy. Magnetic effects on the emission intensity may signal magnetically sensitive chemical population processes and thus provide special insight into the chemical processes occurring in flames.

It seems to be from this point of view that Hayashi and co-workers ${ }^{215}$ have investigated magnetic field effects on the emission from various excited molecular or atomic species produced in flames. Thus it has been found that emission from the sodium $D$ line, observed when hydrogen/oxygen flames are fed by sodium salt solutions, is enhanced by a magnetic field.

The effect was reported to depend strongly on the salt concentration and on the anion. Enhancements up to a factor of 2 have been observed at $1.8 \mathrm{~T}$. This effect has been reinvestigated by Sorokin et al. ${ }^{227}$ using high-resolution spectroscopy. It was shown that the MFE is especially pronounced in the region of $\mathrm{Na}$ (or $\mathrm{Cs}$ ) vapor pressure, where line reversions occur due to resonant reabsorption of the emitted light. The magnetic-field-induced enhancement of integrated emission intensity is due to a reduction of this line reversion by the Zeeman broadening of the lines.

A quenching of emission has been found for the emitting species $\mathrm{HPO}^{*}$ and $\mathrm{SnH}^{*}$, obtained in hydro- 
gen/oxygen flames when aerosols of aqueous solutions of $\mathrm{NH}_{4} \mathrm{HPO}_{4}$ or $\mathrm{SnCl}$ are introduced. ${ }^{218}$ Since this effect was independent of the conditions of combustion, it is thought to be specific for a magnetic quenching of the respective fluorescences and not to reflect a magnetic field effect on the population kinetics.

Emissions from the $\mathrm{OH}$ radical in oxygen/propane or oxygen/hydrogen flames have been shown to be magnetic field dependent, too. ${ }^{216,217}$ There is an increase in intensity of about $30 \%$ at $1.8 \mathrm{~T}$ of the emission from particular rotational levels of the $\mathrm{OH}$ radical.

Light emission from NO radicals produced in a microwave discharge in a nitrogen/oxygen mixture is also greatly decreased in a magnetic field. ${ }^{219}$ Measurements with high spectral resolution have shown, however, that only emission from the $v^{\prime}=0$ vibrational level is quenched ( $-80 \%$ at $1.8 \mathrm{~T}$, low-field saturation, $B_{1 / 2} \approx$ $0.2 \mathrm{~T}$ ), whereas emission from $v^{\prime}=1,2$ is even slightly enhanced, without saturating below $1.8 \mathrm{~T}$.

\section{Photochemical Change in the Gas Phase}

It is clear that when magnetic field effects enhance photophysical deactivation pathways, this will affect the efficiency of chemical reactions wherein the excited state may be involved. Three examples of Table 5 belong to this case: The photoaddition of $\mathrm{SO}_{2}$ to pentane $\mathrm{e}^{128}$ and the photodecomposition of deuteriated formaldehyde ${ }^{128}$ and of glyoxal. ${ }^{220}$ On the other hand, the trans-cis isomerizations of butene sensitized by iodine in the gas phase ${ }^{127}$ involves a true magnetic effect on chemical kinetics, namely predissociation of iodine into two iodine atoms. Since these catalyze trans-cis isomerization, an increase of their yield in a magnetic field will also accelerate trans-cis isomerization kinetics. This effect seems to be the first detection of the iodine predissociation effect from the side of the products.

Unless high pressures are used, cage effects do not occur in the gas phase. ${ }^{224}$ Therefore the radical pair mechanism, as common as it may be in the liquid phase, is usually not of importance in the gas phase. This does, however, not apply if biradicals are concerned. There is so far only one example in the literature ${ }^{129}$ where a magnetic field effect on a photochemical reaction involving a biradical intermediate has been reported for the gas phase. The biradical originating from a Norrish type I reaction of cycloheptanone may undergo a decarbonylation reaction (corresponding to the escape channel of the radical pair mechanism) in competition with regeneration of the starting material or formation of 6-heptenal. The latter process requires a multiplicity change from triplet to singlet in the radical pair, which is impeded in a magnetic field. Accordingly, a decrease of the recombination yield in a magnetic field is observed. Prior to this work spin correlation effects had been detected in this system by the CIDNP effect. ${ }^{221}$

The magnetic-field-dependent predissociation reaction of $\mathrm{Cs}_{2}$ investigated by Kato and Onomichi ${ }^{222}$ is remarkable in that the $\mathrm{Cs}$ atoms originating in their excited ${ }^{2} \Pi_{3 / 2}$ state show a nonstatistical population of their magnetic sublevels. This effect is attributed to different Franck-Condon factors between the bound ${ }^{1} \Pi_{u}$ state and the Zeeman sublevels of the dissociative ${ }^{3} \Sigma^{+}{ }_{u}$ state of the $\mathrm{Cs}_{2}$ molecule.

\section{B. In the Solld State}

There are only a few cases where magnetic field effects on chemical changes in the solid state have been reliably reported.

Leung and El-Sayed ${ }^{279}$ investigated the biphotonic decomposition of pyrimidine at $1.6 \mathrm{~K}$. The reaction mechanism is described by the scheme

$$
\mathrm{S}_{0} \underset{\beta_{i}}{\stackrel{\alpha_{i} I}{\longrightarrow}} \mathrm{T}_{1} \stackrel{\gamma_{i} I}{\longrightarrow} \text { decomposition }
$$

where $I$ denotes the photolyzing light flux and $\alpha_{i}, \beta_{i}$, and $\gamma_{i}$ are triplet-sublevel-specific rate coefficients. At 1.6 $\mathrm{K}$ the $\mathrm{T}_{1}$ sublevels are kinetically isolated from each other. A MFE on the overall rate of decomposition ensues from the recoupling of $T_{1}$ substates in a magnetic field together with the fact that the decay coefficients $\beta_{i}$ and $\gamma_{i}$ are unequal for different $i$ (triplet mechanism). The reaction also shows RYDMR effects.

In a similar investigation Dellinger et al. ${ }^{280}$ showed that the triplet decay of solid dimethyl-s-tetrazine, which at $4.2 \mathrm{~K}$ is exclusively due to the process

$$
\left(\mathrm{H}_{3} \mathrm{C}-(\underset{\mathrm{N}-\mathrm{N}}{\mathrm{N}=\mathrm{N}})-\mathrm{CH}_{3}\right)^{*}-2 \mathrm{CH}_{3} \mathrm{CN}+\mathrm{N}_{2}
$$

1

is sublevel selective $\left(k_{y} \approx k_{z} \approx 10^{3} k_{x}\right)$. This causes a magnetic-field-dependent overall decay rate of the triplet. In this case the triplet substates are assumed to be in thermal equilibrium; however, due to the large ZFS and the low temperature, a significant thermal polarization is obtained, so that the kinetic sublevel differences do not average out.

Mori et al. ${ }^{281}$ found an increase of the initial rate and yield of radiation-induced polymerization of acrylonitrile, when increasing a magnetic field from 0 to 0.5 $\mathrm{T}$ at $77 \mathrm{~K}$. In the case of radiation-induced solid-state polymerization of acetaldehyde, however, a decrease of the yield was observed. ${ }^{282}$ No definitive mechanism seems to have emerged from these investigations.

A MFE on the rate of thermal decomposition of barium azide (increase) and silver oxalate (decrease) has been reported by Russian workers. ${ }^{283}$

There may be more reports in the less accessible literature. However, it can be said that the field of solid-state chemistry is not very rich with clear-cut examples of magnetic-field-dependent reactions. On the other hand, there is a rich choice of physical and photophysical phenomena in molecular crystals, such as luminescence, radioluminescence, and photoconductivity, which have been shown to be caused by a few basic mechanisms, the awareness of which should be useful to the photochemist interested in MFEs.

Most of the investigations mentioned were published between 1965 and 1978, and several reviews have appeared during that period. , $7,12,13,16,25_{\text {In most of these }}$ the mechanistic principles are nicely explained. The reviews by Sokolik and Frankevich ${ }^{12}$ (1973), Swenberg and Geacintov ${ }^{13}$ (1973), and Geacintov and Swenberg ${ }^{25}$ (1978) are rather comprehensive. Therefore we have confined ourselves to provide a condensed access to the field in the form of Table 6, which also includes more recent work not covered in the above-mentioned reviews.

Most of the studies listed in Table 6 deal with organic 
TABLE 6. Magnetic Field Effects on Luminescence and Photoconductivity in Molecular Crystals

\begin{tabular}{|c|c|c|c|}
\hline compd & MF-dependent observation ${ }^{a}$ & mechanism suggested & ref \\
\hline naphthalene & $\begin{array}{l}\mathrm{P} \text {, steady state intensity and } \\
\text { decay rates }\end{array}$ & triplet mechanism & Sixl and Schwoerer ${ }^{228}$ \\
\hline 1,4-dibromonaphthalene & $\mathrm{P}$ at $2 \mathrm{~K}$ & $\begin{array}{l}\mathrm{T}+\mathrm{T} \rightarrow \mathrm{S}_{0} \text { as } \mathrm{T} \text {-quenching } \\
\text { mechanism }\end{array}$ & Vankan and Veeman ${ }^{288}$ \\
\hline \multirow[t]{6}{*}{ anthracene } & $\mathrm{PC}$ & $\begin{array}{l}\text { MFEs on efficiency of charge } \\
\text { carrier production }\end{array}$ & $\begin{array}{c}\text { Frankevich and Balabanov, } \\
\text { Frankevich et al.,230 } \\
\text { Frankevich and Sokolik } \\
\end{array}$ \\
\hline & dark current & $\begin{array}{l}\text { magnetic change of activation } \\
\text { energy }\end{array}$ & Morgan and Pethig ${ }^{232}$ \\
\hline & $\mathrm{PC}$, high-field resonances & triplets involved & $\begin{array}{l}\text { Delannoy and Schott, } \\
\text { Rusin et al. }\end{array}$ \\
\hline & PC, with hole-injecting electrodes & $\mathrm{T}+\mathrm{D}_{\mathrm{tr}}^{+} \rightarrow \mathrm{S}_{0}+\mathrm{D}_{\mathrm{mob}}^{+}$ & $\begin{array}{l}\text { Geacintov et al., }{ }^{233} \text { Frankevich } \\
\text { and Sokolik, }{ }^{234} \text { Frankevich, }{ }^{173} \\
\text { Frankevich et al., }{ }^{241} \\
\text { Bouchriha et al. }{ }^{235}\end{array}$ \\
\hline & $\begin{array}{l}\text { DF, field-dependent orientation } \\
\text { resonances }\end{array}$ & $\mathrm{T}+\mathrm{T} \rightarrow \mathrm{S}_{1}$ & $\begin{array}{l}\text { Johnson et al., }{ }^{236,242} \text { Johnson and } \\
\text { Merrifield, }{ }^{73} \text { Frankevich et al., }{ }^{240} \\
\text { Fourney and Delacote }{ }^{243}\end{array}$ \\
\hline & $\begin{array}{l}\text { DF, low temperature } \\
\text { PF }\end{array}$ & $\begin{array}{l}\mathrm{T}+\mathrm{T} \rightarrow \mathrm{S}_{1} \\
\mathrm{~S}_{1}(\text { hot }) \rightarrow \mathrm{T}+\mathrm{T}\end{array}$ & $\begin{array}{l}\text { Smith and Hughes, }{ }^{244} \text { Soos }^{245} \\
\text { Klein et al., }{ }^{237,238} \text { Arnold }^{246}\end{array}$ \\
\hline anthracene $\left(h_{10}, d_{10}\right)$ & $\mathrm{PF}$, excitation spectrum of MFE & $\begin{array}{l}\mathrm{S}_{1} \rightarrow \mathrm{T}+\mathrm{T} \text {, threshold of } \\
\mathrm{S}_{\mathrm{I}} \text {-vibrational energy }\end{array}$ & Albrecht et al. ${ }^{239}$ \\
\hline anthracene & $\begin{array}{l}\text { EL, } \mathrm{h}^{+} \text {and } \mathrm{e}^{-} \text {injection } \\
\text { EL, separation of PF and DF } \\
\alpha \text {-particle scintillation: PF, DF } \\
\alpha \text {-particle scintillation: DF } \\
\beta \text {-particle scintillation: DF } \\
\text { scintillations from various } \\
\text { high-energy radiation } \\
(\alpha, \beta, p, \text { VUV })\end{array}$ & $\begin{array}{l}\text { fission and fusion } \\
\text { fission and fusion } \\
\mathrm{T}+\mathrm{T} \rightarrow \mathrm{S}_{1} \\
\mathrm{~T}+\mathrm{T} \rightarrow \mathrm{S}_{1} \\
\mathrm{~T}+\mathrm{T} \rightarrow \mathrm{S}_{1} \\
\quad \mathrm{D}^{+}+\mathrm{D}^{-} \rightarrow \mathrm{S}_{1}\end{array}$ & $\begin{array}{l}\text { Frankevich and Rumyantsev }{ }^{247} \\
\text { Schwob and Williams } \\
\text { Geacintov et al. }{ }^{250} \\
\text { Arnold et al. }{ }^{251} \\
\text { Ern and McGhie } \\
\text { Klein and Voltz, }{ }^{253} \\
\quad \text { Klein et al. }{ }^{254-256}\end{array}$ \\
\hline $\begin{array}{l}\text { anthracene/tetracene } \\
\text { (host/guest) }\end{array}$ & $\begin{array}{l}\text { DF after X-ray preirradiation } \\
\text { DF, spectral separation of host } \\
\text { and guest }\end{array}$ & $\begin{array}{l}\mathrm{T}+\mathrm{D}^{+} \rightarrow \mathrm{S}_{1}+\mathrm{D}^{+} \\
\mathrm{T}_{\mathrm{A}}+\mathrm{T}_{\mathrm{T}} \rightarrow \mathrm{S}_{\mathrm{T}} \text { (heterofusion) }\end{array}$ & $\begin{array}{l}\text { Ern and Merrifield }{ }^{285} \\
\text { Groff et al., }{ }^{286,289} \text { Chabr et al. }{ }^{257}\end{array}$ \\
\hline 9,10 -dichloroanthracene & $\begin{array}{l}\text { DF, } \mathrm{PC} \text { : orientation dependent } \\
\text { resonances }\end{array}$ & $\begin{array}{l}\mathrm{T}+\mathrm{T} \rightarrow \mathrm{S}_{1} \\
\mathrm{~T}+\mathrm{D}_{\mathrm{tr}}^{+} \rightarrow \mathrm{S}_{1}+\mathrm{D}_{\text {mob }}^{+}\end{array}$ & Kotani ${ }^{287}$ \\
\hline \multirow{8}{*}{$\begin{array}{l}\text { 9,10-diphenylanthracene } \\
\text { tetracene }\end{array}$} & $\mathrm{DF}$ & $\mathrm{T}+\mathrm{T} \rightarrow \mathrm{S}_{1}$ & Merrifield ${ }^{269}$ \\
\hline & $\begin{array}{l}\mathrm{PC} \text {, high-field resonances } \\
\mathrm{PC} \text { with hole injection }\end{array}$ & $\begin{array}{l}\text { MFE on efficiency of charge } \\
\text { carrier production } \\
\text { triplets involved } \\
\mathrm{S}_{1} \rightarrow \mathrm{T}+\mathrm{T} \text { or } \mathrm{T}+\mathrm{T} \rightarrow \mathrm{S}_{1} \text {, } \\
\text { depending on exciton } \\
\text { density }\end{array}$ & $\begin{array}{l}\text { Frankevich and Balabanov, } \\
\text { Frankevich et al. }{ }^{230} \\
\text { Delannoy and Schott } \\
\text { Geacintov et al., }{ }^{233} \text { Frankevich }^{173}\end{array}$ \\
\hline & PF, MFD, and high-field resonances & $\mathrm{S}_{1} \rightarrow \mathrm{T}+\mathrm{T}$ & Merrifield et al. ${ }^{258}$ \\
\hline & PF, temperature dependence & $\mathrm{S}_{1} \rightarrow \mathrm{T}+\mathrm{T}$ & Geacintov et al. ${ }^{259}$ \\
\hline & PF, variation of excitation intensity & $\begin{array}{l}\text { fission, superimposed by } \\
\text { fusion at high exciton } \\
\text { densities }\end{array}$ & Pope et al. ${ }^{260}$ \\
\hline & $\begin{array}{l}\text { PF, variation of excitation wavelength } \\
\text { PF }\end{array}$ & fission and fusion & $\begin{array}{l}\text { Groff et al. }{ }^{261,262} \\
\text { Moller and Pope }\end{array}$ \\
\hline & $\mathrm{EL}, \mathrm{h}^{+}$and $\mathrm{e}^{-}$injection & $\begin{array}{l}\mathrm{S}_{1} \rightarrow \mathrm{T}+\mathrm{T} \\
\mathrm{T}+\mathrm{D}^{+} \rightarrow \mathrm{S}_{0}+\mathrm{D}^{+}\end{array}$ & Kalinowski and Godlewski ${ }^{264}$ \\
\hline & $\begin{array}{l}\alpha \text {-particle scintillation, temperature } \\
\text { dependence }\end{array}$ & fission and fusion effects & Geacintov et al. ${ }^{250}$ \\
\hline \multirow[t]{2}{*}{$\begin{array}{l}\text { tetracene/pentacene } \\
\text { (host/guest) }\end{array}$} & $\begin{array}{l}\text { PF, spectral separation of host } \\
\text { and guest emission }\end{array}$ & homo- and heterofission & Geacintov et al. ${ }^{265}$ \\
\hline & $\begin{array}{l}\mathrm{PF} \text { and } \mathrm{DF} \text {, at high excitation } \\
\text { intensity }\end{array}$ & heterofusion & Kalinowski and Godlewski ${ }^{266}$ \\
\hline $\begin{array}{l}\text { tetracene/2,3-benzocarbazole } \\
\text { (host/guest) }\end{array}$ & PF, high dopant concentration & $\begin{array}{l}\text { geminate } T+T \text { fusion: } \\
\text { "exciton caging" }\end{array}$ & Arnold et al. ${ }^{267,268}$ \\
\hline pyrene & DF & $\mathrm{T}+\mathrm{T} \rightarrow \mathrm{S}_{1}$ & Yarmus et al. ${ }^{270}$ \\
\hline fluorene/pyrene (host/guest) & DF, ESR & $\begin{array}{l}\mathrm{T}+\mathrm{T} \rightarrow \mathrm{S}_{1},(\mathrm{TT})-\mathrm{ESR} \\
\text { detected }\end{array}$ & Pekcan $^{271}$ \\
\hline$\alpha$-perylene, $\beta$-perylene & PF, MFD of excitation spectra & $\begin{array}{l}\text { determination of fission } \\
\text { threshold; blue shift in } \\
\text { excimer forming } \alpha \text {-perylene }\end{array}$ & Albrecht et al. ${ }^{239}$ \\
\hline rubrene & DF & $\mathrm{T}+\mathrm{T} \rightarrow \mathrm{S}_{1}$ & Frankevich et al. ${ }^{272}$ \\
\hline $\begin{array}{l}\text { poly }(N \text {-vinylcarbazole) (with } \\
\text { dimethyl terephthalate as } \\
\text { dopant) }\end{array}$ & $\begin{array}{l}\mathrm{PC} \text { and } \mathrm{PF} \text { from exciplex, both } \\
\text { with positive MFD }\end{array}$ & $\begin{array}{l}\text { charge carrier generation } \\
\text { more efficient from singlet } \\
\text { exciplex }\end{array}$ & Okamoto et al. ${ }^{273}$ \\
\hline \multirow[t]{2}{*}{$\begin{array}{l}\text { anthracene/dimethylpyro- } \\
\text { mellitimide EDA complex }\end{array}$} & $P F$ and $D F$ & $\begin{array}{l}\Delta g \text { mechanism in Wannier } \\
\text { exciton and } \mathrm{T}+\mathrm{T} \rightarrow \mathrm{S}_{1}\end{array}$ & Frankevich et al..$^{274}$ \\
\hline & DF, multiresonant MFD & $\begin{array}{l}\mathrm{T}+\mathrm{T} \rightarrow \mathrm{S}_{1} \text {, level crossing of } \\
\text { different } M_{s} \text { levels } \\
\text { (exchange interaction) }\end{array}$ & Lesin et al. ${ }^{275}$ \\
\hline $\begin{array}{l}\text { anthracene/pyromellitic } \\
\text { dianhydride EDA complex }\end{array}$ & $\begin{array}{l}\text { PC, detailed } B_{0} \text { and orientation } \\
\text { dependence }\end{array}$ & $\begin{array}{l}\mathrm{T}+\mathrm{D}_{\mathrm{tr}}^{+} \rightarrow \mathrm{S}_{0}+\mathrm{D}^{+}{ }_{\text {mob }} \\
\text { complete } \mathrm{ZFS} \text {-tensor } \\
\text { evaluation }\end{array}$ & Ziegler and $\mathrm{Karl}^{109}$ \\
\hline
\end{tabular}


TABLE 6 (Continued)

\begin{tabular}{|c|c|c|c|}
\hline compd & MF-dependent observation ${ }^{a}$ & mechanism suggested & ref \\
\hline Pt(phthalocyanine) & $\mathrm{P}$, decay at $4.2 \mathrm{~K}, B_{0}$ up to $10 \mathrm{~T}$ & $\begin{array}{c}\mathrm{T}+\mathrm{T} \rightarrow \mathrm{S}_{1} \text {, high-field maximum } \\
\text { of } \mathrm{MFD} \text { due to } 8.5-\mathrm{cm}^{-1} \mathrm{ZFS}\end{array}$ & Kaneto et al. ${ }^{276}$ \\
\hline $\begin{array}{l}\mathrm{W}(\mathrm{CO})_{5} \text { (pyridine) } \\
\mathrm{Ba}\left[\mathrm{Pt}(\mathrm{CN})_{4}\right]\end{array}$ & $\begin{array}{l}\mathrm{P}, \text { low-temperature spectra, new } \\
\text { magnetically induced transitions }\end{array}$ & $\begin{array}{l}\text { magnetic mixing of spin-orbit split } \\
\text { triplet substates }\end{array}$ & Gliemann $^{277}$ \\
\hline $\begin{array}{l}\mathrm{Cu}^{+} \text {in } \mathrm{LiCl}, \mathrm{NaF}, \mathrm{NaCl}, \\
\mathrm{NaBr}, \mathrm{KCl}\end{array}$ & $\mathrm{P}$, lifetime at $4.2 \mathrm{~K}$ & $\begin{array}{l}\text { magnetic mixing of spin-orbit split } \\
\text { triplet substates }\end{array}$ & Payne et al. ${ }^{278}$ \\
\hline
\end{tabular}

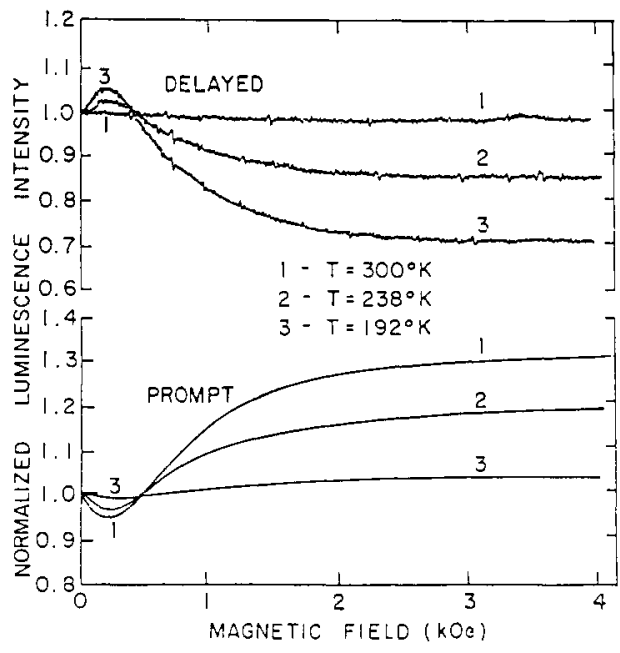

Figure 10. Experimental MFD curves of delayed and prompt fluorescence in a tetracene crystal. The field was oriented at $-20^{\circ}$ with respect to the $b$ axis in the $a b$ plane of the crystal. Reprinted from ref 261 with kind permission of R. E. Merrifield; copyright 1970 Elsevier Science Publishers B.V.

molecular crystals, with particular emphasis on anthracene and tetracene. The reactive species of interest are singlet and triplet excitons and paramagnetic (doublets) charge carriers such as electrons or holes $\left(\mathrm{e}^{-}\right.$, $\left.\mathrm{h}^{+}\right)$, which may be mobile or trapped $\left(\mathrm{D}_{\text {mobs }}^{-}, \mathrm{D}^{+}{ }_{\text {mob }}, \mathrm{D}^{-}\right.$tr, $\left.\mathrm{D}^{+}{ }_{\mathrm{tr}}\right)$. Singlet and triplet excitons may be populated in a direct optical process $\left(\mathrm{S}_{0} \rightarrow \mathrm{S}_{1}, \mathrm{~S}_{0} \rightarrow \mathrm{T}_{1}\right)$ or may be mutually converted into each other $\left(\mathrm{S}_{1}+\mathrm{S}_{0} \rightarrow \mathrm{T}+\right.$ $\mathrm{T}$, singlet exciton fission, $\mathrm{T}+\mathrm{T} \rightarrow \mathrm{S}_{1}+\mathrm{S}_{2}$, triplet exciton fusion, or triplet-triplet annihilation). They can be also formed in charge recombinations $\left(\mathrm{D}^{+}+\mathrm{D}^{-} \rightarrow\right.$ $\mathrm{S}_{1}$, T). Charge carriers, on the other hand, may be produced by exciton dissociation, by charge injection from suitable electrodes, or by high-energy radiation.

Various techniques have been applied to study magnetic field effects on the interaction dynamics and decay of such species. Triplet exciton lifetimes are most directly probed via the phosphorescence signal. ${ }^{228,276-278,288}$ They may owe their magnetic field sensitivity to the triplet mechanism $228,277,278$ or to triplet-triplet annihilation processes. ${ }^{276,288}$ The first type has been seen in magnetic-field-enhanced phosphorescence $^{228}$ or even in the appearance of new vibrational lines in the emission spectrum as reported by Glie$\operatorname{man}^{277}$ for the transition-metal complex $\mathrm{W}(\mathrm{CO})_{5}$ (pyridine). A recent example of the latter type has been reported by Vankan and Veeman ${ }^{288}$ for 1,4-dibromonaphthalene at $2 \mathrm{~K}$. Here the lifetime of trapped triplet excitons is determined by a $\mathrm{T}-\mathrm{T}$ annihilation process with mobile triplet excitons. At high fields of several tesla the predominant Boltzmann population of the $T$. states causes a reduced probability of ${ }^{1}(\mathrm{TT})$ pair for- mation, which would require encounters of the type $\left(\mathrm{T}_{+} \mathrm{T}_{-}, \mathrm{T}_{-} \mathrm{T}_{+}, \mathrm{T}_{0} \mathrm{~T}_{0}\right)$.

Monitoring the fluorescence emission from singlet excitons, one can detect MFEs on singlet exciton fission and triplet exciton fusion. These are readily understood in the framework of Merrifield's model (cf. section II). The rate constant of the $T+T \rightarrow S_{1}$ process in a random encounter (TT) pair will be largest if the singlet pair state ${ }^{1}(\mathrm{TT})$ is distributed most uniformly over the eigenstates of the pair spin Hamiltonian. This effect is borne out in the MFD of the delayed fluorescence (cf. Figure 10), typically showing an increase at low fields, a sign conversion for fields comparable to the ZFS, and a negative branch at high field. Several maxima in the DF-MFD curve may be formed if the (TT) pair states of different multiplicity are split in zero field. Such exchange splitting is usually negligible for plain aromatic compounds. But evidence for its effect has been provided with crystals of the pyromellitic $N, N^{\prime}$-dimethyldiimide/anthracene EDA complex by Lesin et al. ${ }^{275}$ The additional maxima in this case are thought to be due to level crossings of the ${ }^{1}(\mathrm{TT})_{0}$ with ${ }^{5}(\mathrm{TT})_{-2}$ and ${ }^{5}(\mathrm{TT})_{-1}$ pair states.

A MFD opposite to the delayed fluorescence is to be found for prompt fluorescence, the intensity of which is decreased by the possibility of the reversible fission process

$$
\mathrm{S}_{1} \rightleftarrows \mathrm{TT} \rightarrow \mathrm{T}+\mathrm{T}
$$

Here the dissociation is most effective if the singlet character is evenly distributed over the spin-Hamiltonian eigenstates of the (TT) pair. An example of this behavior is shown in Figure 10. This also provides evidence of the activated nature of the fission process, which predominates at higher temperature. Other evidence for the activation energy required has been obtained from the excitation wavelength dependence of the MFD of prompt fluorescence. ${ }^{239,261,262}$ Albrecht et al. ${ }^{239}$ found a marked difference between $\alpha$-perylene and $\beta$-perylene when determining the onset wavelength for the fission-type MFE. The crystal structure favors excimer formation in $\alpha$-perylene and may thus provide an efficient radiationless decay channel other than the fission process unless higher excitation energies are applied.

In Figure 11 an example of the so-called high-field resonances is shown, occurring at special orientations of the crystal axes to the magnetic field, where a level crossing of the two ${ }^{1,5}(\mathrm{TT})_{m=0}$ states occurs (cf. section II).

Prompt fluorescence and delayed fluorescence induced by exposure of molecular crystals to high-energy radiation also show MFEs of the fission and fusion type. Here, as in the case of electroluminescence, where electrons and holes are injected from suitable electrodes, 


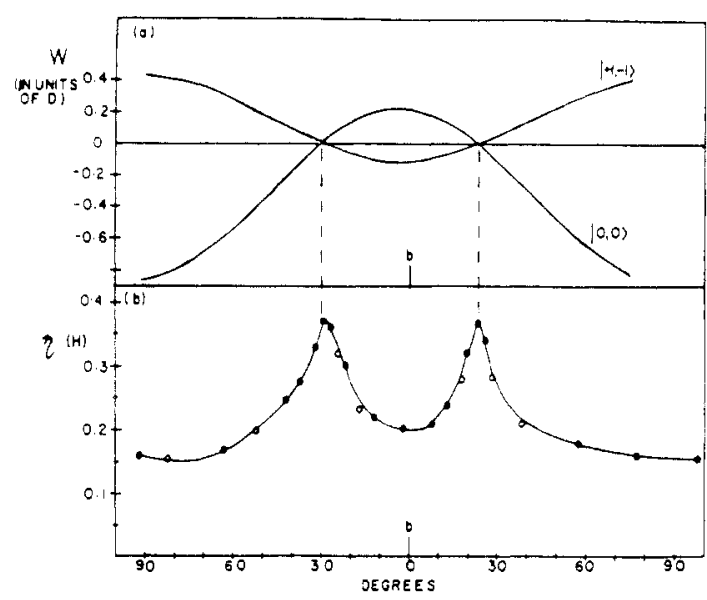

Figure 11. The MFD of tetracene fluorescence as a function of orientation of the magnetic field vector $(4000 \mathrm{G})$ in the $a b$ plane: (a) calculation of triplet-pair-state energies; (b) experimental results, $\eta(H)$ relative enhancement of fluorescence intensity. Reprinted from ref 259 with kind permission of N. Geacintov, M. Pope, and F. Vogel; copyright 1969 American Institute of Physics.

singlet and triplet excitons are produced by electronhole recombination. The latter process, where a pair of doublet states recombines, could also give rise to a characteristic MFD according to the radical pair mechanism. In fact, Frankevich suggested this type of explanation for the dynamics of "weakly bound" CT states (Wannier excitons) where singlet-triplet multiplicity changes might occur according to the $g$ mechanism. 231,274,284 More recent evidence for involvement of the radical pair type mechanism with hfc as the singlet-triplet mixing perturbation has been obtained by Klein et al. ${ }^{254-256}$ from time-resolved luminescence, excited by high-energy radiation. The luminescence intensity at 100-500-ns delay time displayed the typical MFD of the hfc mechanism for geminate electron-hole pairs originating in an overall triplet state. This would indicate that, in order to guarantee the overall singlet spin state of the spur, a triplet exciton is also produced during thermalization of primary ejected electrons:

$$
{ }^{1}\left(\mathrm{e}^{-} \mathrm{h}^{+}\right)^{*}+\mathrm{S}_{0} \rightarrow{ }^{3}\left(\mathrm{e}^{-} \mathrm{h}^{+}\right)+\mathrm{T}_{1}
$$

Other examples where the radical pair type mechanism is operating have been reported for solid-liquid interfaces and will be discussed in section IV.E.

A great many MFEs have been revealed by photoconductivity measurements in organic molecular crystals. These investigations have been pioneered by Frankevich and co-workers (cf. Table 6). As first demonstrated by Geacintov et al., ${ }^{233}$ the effects are predominantly due to the detrapping of doublet charge carriers by interaction with triplet excitons:

$$
\mathrm{T}+{ }^{2} \mathrm{D}_{\mathrm{tr}}^{+} \rightarrow \mathrm{S}_{0}+{ }^{2} \mathrm{D}^{+}{ }_{\text {mob }}
$$

MFEs may be understood as a sequence of spin-selective interactions in the (TD) pairs determining the efficiency of the charge carrier detrapping process. Of course, the effect of TT annihilation will be also seen if this process contributes significantly to the decay of triplet excitons, thus determining their stationary concentration, which will be reflected in the overall rate of charge carrier detrapping.

The MFE on photocurrents, including its orientational dependence, may be used to obtain detailed in-

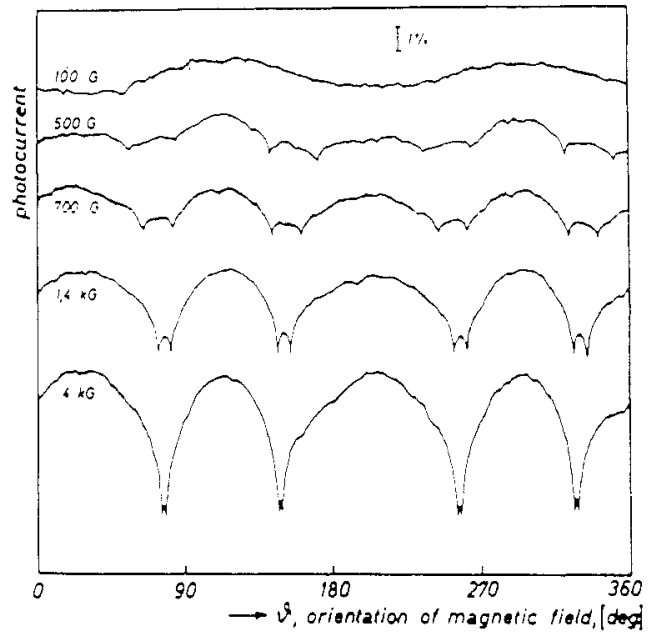

Figure 12. Anisotropy of photocurrent in an anthracene-pyromellitic dianhydride crystal when the magnetic field is rotated in a fixed crystal plane for the field values indicated. The individual curves are shifted relative to each other on the ordinate. The scale of a $1 \%$ modulation of total current is indicated at the top of the figure. Reprinted from ref 109 with kind permission of N. Karl; copyright 1979 Elsevier Science Publishers B.V.

formation on the interacting species involved (TDR and TTR; cf. Table 3). Ziegler and Karl ${ }^{109}$ have used such measurements (cf. Figure 12) for a complete determination of the ZFS tensor of triplet excitons in crystals of the anthracene/pyromellitic dianhydride EDA complex.

In concluding this section, we note that there are also a variety of MFEs in the field of luminescence and photoconductivity of inorganic solids and semiconductors. Some of these are collected in the reviews by Street $^{40}$ and Cavenett ${ }^{117}$ (cf. also section VI.G).

\section{In Homogeneous Llquid Solutions}

By far most of the magnetic field effects on chemical reactions have been found in liquid solutions, and most of them are due to the radical pair mechanism. This results from a rather favorable relation of radical mobility and solvent cage effect in liquids. Whereas in gases separation of radical pairs is very fast and there is practically zero probability of geminate reencounters, in solid phases radical pairs are generally not capable of separating efficiently unless one of the unpaired electrons can move as a conduction band electron or by electron hopping. In liquid phases cage reactions and diffusional separation of radical pairs may take place with rates of similar order of magnitude so that a MFE on the cage recombination may often lead to detectable changes of product ratios. One should be aware, however, that although the radical pair mechanism is really dominating in liquid solutions, magnetokinetic effects in such media can be also due to other pair mechanisms $(\mathrm{T}+\mathrm{T}=\mathrm{T}+\mathrm{D})$ or to the triplet mechanism.

In the present section we deal separately with thermal chemical reactions, photoreactions, and luminescence processes. Other effects in homogeneous liquid solution will be found in sections IV.G (reaction-yield-detected magnetic resonance) and IV.H (magnetic isotope effects). 
TABLE 7. Magnetic Field Effects in Thermal Reactions of Alkali-Metal Alkyls ${ }^{a}$

\begin{tabular}{|c|c|c|c|c|}
\hline $\mathbf{A}-\mathbf{M}^{a}$ & $\mathrm{~B}-\mathrm{X}^{a}$ & MFE characteristics ${ }^{b}$ & anal..$^{c}$ & ref \\
\hline $\begin{array}{c}n-\mathrm{C}_{m} \mathrm{H}_{2 m+1} \mathrm{Li} \\
\left(\mathrm{C}_{2} \mathrm{H}_{5}\right)_{3} \mathrm{Ge}(\mathrm{Li} \\
\mathrm{Na}, \mathrm{K})\end{array}$ & $\begin{array}{l}\mathrm{C}_{6} \mathrm{H}_{5} \mathrm{CH}_{2} \mathrm{Cl} \\
\mathrm{C}_{6} \mathrm{~F}_{5} \mathrm{CH}_{2} \mathrm{Cl} \\
p-\mathrm{FC}_{6} \mathrm{H}_{4} \mathrm{CH} \mathrm{CH}_{2} \mathrm{Cl} \\
\left(\mathrm{C}_{6} \mathrm{~F}_{5}\right)_{2} \mathrm{CHCl} \\
\mathrm{C}_{6} \mathrm{H}_{5} \mathrm{CH}_{2} \mathrm{Cl} \\
\mathrm{C}_{6} \mathrm{H}_{5} \mathrm{CH}_{2} \mathrm{Cl}\end{array}$ & $\begin{array}{l}R^{\mathrm{c}}(2.5 \mathrm{~T})=+15 \% \\
\text { case } 1: B_{\mathrm{s}} \leq 0.1 \mathrm{~T}, R^{\mathrm{c}}(2.5 \mathrm{~T})=+37 \% \text { (hexane), }+54 \% \text { (cyclohexane) } \\
\text { case } 1: B_{\mathrm{g}} \leq 0.2 \mathrm{~T}, R^{\mathrm{c}}(1.8 \mathrm{~T})=+60 \% \\
\text { case } 1,2: B_{\mathrm{M}} \approx 0.1 \mathrm{~T}, R_{\mathrm{M}}^{\mathrm{c}}=+15 \% ; B_{\mathrm{s}} \approx 1.2 \mathrm{~T}, R^{\mathrm{c}}(1.5 \mathrm{~T})=-20 \% \\
R^{\mathrm{c}}(m) / R_{\mathrm{s}}^{\mathrm{c}}(m=4)=0.6,1,1.4,0(m=3,4,5,7), R_{\mathrm{s}}^{\mathrm{c}}=0 \text { for } i-\mathrm{C}_{3} \mathrm{H}_{7} \mathrm{Li} \\
R^{\mathrm{c}}(1.8 \mathrm{~T})=-11 \%(\mathrm{Li}),-25 \%(\mathrm{Na}),-23 \%(\mathrm{~K})\end{array}$ & $\begin{array}{l}{ }^{1} \mathrm{H} \text { NMR } \\
{ }^{19} \mathrm{~F} \text { NMR } \\
{ }^{19} \mathrm{~F} \text { NMR } \\
{ }^{1} \mathrm{H} \text { NMR } \\
{ }^{1} \mathrm{H} \text { NMR } \\
{ }^{1} \mathrm{H} \text { NMR }\end{array}$ & $\begin{array}{l}\text { Sagdeev et al. }{ }^{22,137} \\
\text { Sagdeev et al. }{ }^{22} \\
\text { Podoplelov et al. }{ }^{138} \\
\text { Podoplelov et al. }{ }^{290} \\
\text { Leshina et al. }{ }^{291}\end{array}$ \\
\hline $\begin{array}{l}\left(\mathrm{C}_{2} \mathrm{H}_{5}\right)_{3} \mathrm{Ge}(\mathrm{Na}, \mathrm{K}) \\
n-\mathrm{C}_{4} \mathrm{H}_{9} \mathrm{Li}\end{array}$ & $\begin{array}{l}\mathrm{C}_{6} \mathrm{H}_{5} \mathrm{CH}_{2} \mathrm{Cl} \\
\left(\left(\mathrm{CH}_{3}\right)_{3} \mathrm{CO}\right)_{2}{ }^{d}\end{array}$ & $\begin{array}{l}\text { case 1, 2: } B_{\mathrm{M}} \approx 0.1 \mathrm{~T}, R_{\mathrm{M}}^{\mathrm{c}} \approx+15 \% ; R^{\mathrm{c}}(1 \mathrm{~T}) \approx-15 \% \\
\text { MFD: oscillatory, } R^{\mathrm{c}}(0.1 \mathrm{~T})=-16 \pm 2 \%\end{array}$ & $\begin{array}{l}{ }^{1} \mathrm{H} \mathrm{NMR} \\
\text { GLC }^{c}\end{array}$ & $\begin{array}{l}\text { Taraban et al. }{ }^{292} \\
\text { Kurskii et al. }{ }^{132}\end{array}$ \\
\hline
\end{tabular}

\section{Thermal Reactions}

Thermal reactions in liquid solutions have provided the first examples of MFEs on chemical reaction yields caused by the radical pair mechanism. ${ }^{137}$ Chemically, magnetic-field-dependent thermal reactions mainly fall into two classes: (i) reactions of alkali-metal alkyls with alkyl halides and (ii) decomposition of organic peroxides and endoperoxides.

The cases of the first type are listed in Table 7, with some data characterizing their magnetic field dependence. The reactions, which seem to be the domain of Russian workers, have been reviewed in some detail by Molin et al. ${ }^{22,52}$

The reagents are heated in nonpolar solvents such as hexane or cyclohexane and react according to eq 39

$$
A M+B X-M X+{ }^{1} \overline{A^{*} B^{\circ}}-\underbrace{\text { escape }}_{\text {cage }} A B+B B, A B \text { (39) }
$$

Formation of a singlet-spin-aligned radical pair of a benzyl and an alkyl radical is the primary reaction step. Cage recombination of the radical pair leads to the unsymmetric coupling product $\mathrm{A}-\mathrm{B}$, whereas escape yields the symmetric coupling products $\mathrm{A}-\mathrm{A}$ and $\mathrm{B}-\mathrm{B}$, and the unsymmetric one in statistical ratios. Spin evolution in the primary singlet radical pair leads to triplet-spin alignment, which precludes recombination and hence favors escape product formation.

Soon after the discovery of CIDNP in such systems, ${ }^{293}$ MFEs were found to modify the ratio of cage to escape products, characterized by the product ratio [A-B]/ $[\mathrm{A}-\mathrm{A}]$, which was determined by NMR analysis. The magnetic field dependence has not been measured in full detail in every case but the data are qualitatively consistent with the hyperfine coupling mechanism modified in several cases by the $\Delta g$ mechanism, which is indicated by a sign inversion of the effect between low field and high field.

Theoretical simulation of the $\mathrm{MFEs}^{52}$ is only possible if extremely long cage times are assumed, which cannot be understood on the basis of free diffusion of neutral radicals. An explanation is offered in terms of association of the radical pairs to lithium oligomers, known to exist in such solutions. This situation is in some way reminiscent of the large effects in micellar aggregates discussed below.

It may be of special interest that Table 7 also contains some examples with germanium compounds. Experiments have been performed with samples of natural germanium isotope abundance, i.e., with about $7 \%$ of ${ }^{73} \mathrm{Ge}$, exhibiting a fairly strong hyperfine coupling constant of $24 \mathrm{mT}$. In fact, the MFE is of the case 1,2 type, indicating that at lower fields the radical pair reactivity is influenced by Ge hyperfine coupling, while a strong $\Delta g$ effect predominates at higher fields. These results seem to indicate the feasibility of magnetic isotope separation even for elements heavier than $\mathrm{C}$ and $\mathrm{O}$ (cf. section IV.H).

The MFE on the reaction between $n$-butyllithium and di-tert-butyl peroxide has been investigated by Kurskii et al. ${ }^{132}$ Here the singlet radical pair ${ }^{1}(n$ $\left.\mathrm{C}_{4} \mathrm{H}_{9}{ }^{\circ} \mathrm{OC}\left(\mathrm{CH}_{3}\right)_{3}\right)$ is the primary geminate pair. The decrease of cage product formation is mainly attributed to the $\Delta g$ mechanism. A qualitative simulation of the somewhat oscillatory MFD could be achieved by taking into account the influence of spin relaxation using a formalism developed by Kubarev et al. ${ }^{294}$ (cf. also section V.C).

Thermal decomposition of several endoperoxides has been studied in magnetic fields by Turro and co-workers. ${ }^{140,147,295}$ These experiments have been reviewed by Gould et al..$^{53}$ Thermolysis of the endoperoxides yields molecular oxygen originating to some extent as singlet oxygen, which may be determined quantitatively by trapping it in a reaction with tetracyclone (2).<smiles>CC(=O)C(=C(C(=O)c1ccccc1)C(=O)c1ccccc1)C(c1ccccc1)c1ccccc1</smiles>

Whereas the singlet oxygen yield with the 9,10endoperoxide 4 shows a strongly negative MFD between 1 and $1.4 \mathrm{~T}(R=-6.2 \%,-15.6 \%$, and $-28 \%$ at respectively $0.95,1.15$, and $1.35 \mathrm{~T}$ ), the singlet oxygen yield in the case of the 1,4-endoperoxide 5 is magnetic field independent. These findings correspond with<smiles></smiles><smiles>[R]C12CCC([R])(C1)c1c2c(-c2ccccc2)c(-c2ccccc2)c2ccccc12</smiles>

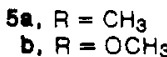

other mechanistic evidence ${ }^{141}$ indicating that the decomposition occurs by a concerted mechanism for 5 and by a diradical mechanism for 4 . The MFE in this case is attributed to the influence of the $\Delta g$ mechanism, favoring intersystem crossing in the singlet diradical species. Also, ${ }^{17} \mathrm{O}$ magnetic isotope effects have been discovered, ${ }^{147}$ which will be commented on below (cf. section IV.H). 
TABLE 8. Photochemical Magnetic Field Effects in Homogeneous Solution

\begin{tabular}{|c|c|c|c|c|}
\hline reactants ${ }^{a}$ & solvent $^{b}$ & $\begin{array}{c}\text { method of } \\
\text { observation }^{c}\end{array}$ & characteristics of $\mathrm{MFE}^{d}$ & ref \\
\hline \multicolumn{5}{|c|}{ Electron-Transfer Reactions with Excited Singlets } \\
\hline${ }^{1} \mathrm{Py} *+$ DMDMA & $\mathrm{MeOH}$ & LFP, DF & $R\left(\left[{ }^{3} \mathrm{Py}\right]\right)$, case $1: B_{\mathrm{s}} \approx 15 \mathrm{mT}, R_{\mathrm{s}} \approx-14 \%$ & Schulten et al. ${ }^{302}$ \\
\hline \multirow[t]{3}{*}{${ }^{1} \mathrm{Py}^{*}+\mathrm{DEA}$} & $\mathrm{MeOH}$ & TP-LFP & $\underset{\mathrm{mT}}{\left.R\left({ }^{3} \mathrm{Py}\right], 8 \mathrm{~ns}, 20 \mathrm{mT}\right)} \approx-10 \%, B_{1 / 2} \approx 4$ & Michel-Beyerle et al. ${ }^{15}$ ? \\
\hline & $\begin{array}{l}\mathrm{ACN}, 2-\mathrm{PrOH} \\
i \text {-AmOH }\end{array}$ & $\mathrm{PC}$ & $\begin{array}{l}R\left(i_{\mathrm{p}}\right), \text { case } 1: \quad R_{\mathrm{g}}=4.5 \%(\mathrm{ACN}),-2.4 \% \\
\quad(2-\mathrm{PrOH}),-0.8 \%(i-\mathrm{AmOH})\end{array}$ & Fedotova et al. ${ }^{762}$ \\
\hline & $\begin{array}{l}2-\mathrm{PrOH} / \mathrm{ACN} \\
\text { mixed solvent }\end{array}$ & $\mathrm{PC}$ & $R\left(i_{\mathrm{p}}\right)$ sign inversion at $1 \% \mathrm{ACN}$ & Frankevich and Fedotova ${ }^{763}$ \\
\hline \multirow[t]{2}{*}{$\begin{array}{l}{ }^{1} \mathrm{Py}^{*}+\mathrm{DMA}(h, d) \\
{ }^{1} \mathrm{Py}^{*}+\mathrm{DMDMA}\end{array}$} & $\begin{array}{l}\text { ACN, DMF, } \\
\text { MeOH, EtOH }\end{array}$ & LFP, DF & $\begin{array}{l}R\left(\left[{ }^{3} \mathrm{Py}\right]\right), \text { case } 1: R_{\mathrm{s}} \text { solvent dependent, } \\
B_{1 / 2} \approx 5.5 \mathrm{mT}(h), 2.7 \mathrm{mT}(d)\end{array}$ & Werner et al., ${ }^{303}$ Weller $^{304}$ \\
\hline & $\mathrm{MeOH}$ & DF & $\begin{array}{l}\left.R\left({ }^{3} \mathrm{Py}\right]\right), B_{1 / 2} \text { dependent on [donor], } \mathrm{e}^{-}- \\
\text {hopping effect }\end{array}$ & Nolting et al. ${ }^{305}$ \\
\hline \multirow[t]{2}{*}{${ }^{1} \mathrm{Py}^{*}+\mathrm{DMA}$} & $\mathrm{ACN}$ & TP-LFP & $R\left(\left[{ }^{3} \mathrm{Py}\right]\right), R\left(\left[\mathrm{Py}^{-}\right]\right)$ & Treichel et al. ${ }^{159}$ \\
\hline & $\mathrm{MeOH}$ & TP-LFP & $\begin{array}{l}R\left(\left[{ }^{3} \mathrm{Py}\right]\right), B_{1 / 2} \text { dependent on }[\mathrm{DMA}] \text { and } \\
\text { delay time: lifetime uncertainty broad- } \\
\text { ening }\end{array}$ & Staerk et al. ${ }^{307}$ \\
\hline $\begin{array}{l}\text { Py* }\left(\mathrm{Py}-d_{10}\right)+\mathrm{DCNB}(h, d) \\
p-\mathrm{F}-\mathrm{DMA}(h, d), \mathrm{DMT} \\
\text { DMA }(h, d), \mathrm{DMDMA}\end{array}$ & $\mathrm{ACN}$ & DF & $\begin{array}{l}R\left(\left[^{3} \mathrm{Py}\right]\right), B_{1 / 2} \text { correlation with hfc con- } \\
\text { stants }\end{array}$ & Weller et al. ${ }^{306}$ \\
\hline \multirow[t]{2}{*}{$\begin{array}{l}{ }^{1} \mathrm{Py}^{*}-\left(\mathrm{CH}_{2}\right)_{n}-\mathrm{DMA} \\
6 \leq n \leq 12\end{array}$} & $\mathrm{ACN}$ & TP-LFP & $R\left(\left[{ }^{3} \mathrm{Py}\right]\right)$, case $1,3: B_{\mathrm{M}} n$ dependent & $\begin{array}{l}\text { Weller et al., }{ }^{308} \\
\text { Staerk et al. }\end{array}$ \\
\hline & & $F$ & $R\left(\Phi_{\mathrm{f}}\right.$ exciplex $), R_{\mathrm{s}} \leq+50 \%$ & Staerk et al. \\
\hline${ }^{1} \mathrm{Py} *+$ trans-stilbene $\left(h_{10}, f_{10}\right)$ & $\mathrm{ACN}$ & ${ }^{1} \mathrm{H}$ NMR & $\begin{array}{l}\left.R\left(\Phi_{\text {trans-cis }}\right), \mathrm{H}_{10} \text { \{case } 1: R_{\mathrm{a}} \approx-30 \%\right\}, \mathrm{F}_{10} \\
\{\text { case } 1,2)\}\end{array}$ & Leshina et al. ${ }^{310,311}$ \\
\hline${ }^{1} \mathrm{Ac} *\left(h_{10}, d_{10}\right)+\mathrm{DEA}$ & $\mathrm{ACN}$ & $\begin{array}{l}\mathrm{CP}, \mathrm{MF} \\
\text { modulation }\end{array}$ & $\begin{array}{l}R\left(\left[\mathrm{Ac}{ }^{-}\right]\right), \text {case } 1: B_{1 / 2}\left(h_{10}\right)=7.5 \mathrm{mT}, B_{\mathrm{s}} \approx \\
\quad 12 \mathrm{mT}, R_{\mathrm{s}} \approx+1.3 \% ; B_{1 / 2}\left(d_{10}\right)=6.2 \mathrm{mT}\end{array}$ & Bube et al. ${ }^{112}$ \\
\hline \multirow[t]{2}{*}{${ }^{1} \mathrm{Ac} *+\mathrm{DMA}$} & $\mathrm{ACN}$ & TP-LFP & $\begin{array}{l}R\left(\left[{ }^{3} \mathrm{Ac}\right]\right), B_{1 / 2} \text { dependence on probe pulse } \\
\text { delay time }\end{array}$ & Michel-Beyerle et al. ${ }^{312}$ \\
\hline & & & $\begin{array}{l}B_{1 / 2} \text { dependence on }[\mathrm{DMA}], \mathrm{e}^{-} \text {-hopping } \\
\text { effect }\end{array}$ & Krüger et al. ${ }^{313}$ \\
\hline${ }^{1}(9-\mathrm{Me}-\mathrm{Ac}) *+\mathrm{DMA}$ & $\mathrm{ACN}$ & TP-LFP & $\begin{array}{l}R\left(\left[^{3} \mathrm{Ac}\right]\right), \text { case } 1: B_{1 / 2} \approx 8 \mathrm{mT}, B_{\mathrm{s}} \approx 30 \\
\quad \mathrm{mT}, R_{\mathrm{s}} \approx-12 \%\end{array}$ & Treichel et al. ${ }^{159}$ \\
\hline${ }^{1} \mathrm{Ac} *(h, d)+\mathrm{DMA}(h, d)$ & $\mathrm{ACN}$ & TP-LFP & $\begin{array}{l}B_{1 / 2} \text { dependence on [DMA], } \mathrm{e}^{-} \text {-hopping } \\
\text { with } h / d \text { isotope effect }\end{array}$ & Krüger et al. ${ }^{314}$ \\
\hline $\begin{array}{l}{ }^{1} \text { Phen"- }\left(\mathrm{CH}_{2}\right)_{n} \text {-DMA } \\
n=3,6,8,10\end{array}$ & $\mathrm{ACN}$ & $\mathrm{F}$ & $\begin{array}{l}\left.R\left(I_{\mathrm{f}} \text { exciplex }\right), n=10 \text { case } 1, R_{\mathrm{s}} \approx+50 \%\right\} \\
\quad n<10\{\text { case } 1,3\}\end{array}$ & Tanimoto et al. ${ }^{351}$ \\
\hline \multirow[t]{2}{*}{$\begin{array}{l}{ }^{1} \text { TMPDA* }^{*} \\
\text { photoionization }\end{array}$} & 2-PrOH & $\mathrm{PC}$ & $\begin{array}{l}R\left([\text { ions] }), \text { case } 1, \text { MFD: } B_{1 / 2} \approx 130 \mathrm{mT}, R_{\mathrm{s}}\right. \\
\quad \approx+15 \%, \text { relaxation mechanism }\end{array}$ & Tanimoto et al. ${ }^{172}$ \\
\hline & \multicolumn{3}{|c|}{ Electron-Transfer Reactions with Excited Triplets } & \\
\hline${ }^{3}$ fluorenone $+\mathrm{DABCO}$ & $\begin{array}{l}\text { propylene } \\
\text { carbonate }\end{array}$ & LFP & $\begin{array}{l}R\left[\mathrm{~F}^{\circ-}\right], \text { case } 1: B_{\mathrm{s}} \approx 27 \mathrm{mT} R_{\mathrm{s}} \approx+20 \% \\
\quad(223 \mathrm{~K}), \text { temp } / \text { visc dependence }\end{array}$ & Periasamy and Linschitz ${ }^{315}$ \\
\hline $\begin{array}{l}{ }^{3} \mathrm{TH}^{+}(6 \mathrm{~S})+ \\
\quad(o, m, p)-\mathrm{I}-\mathrm{An}, p-\mathrm{Br} \cdot \mathrm{An}\end{array}$ & $\mathrm{MeOH}$ & LFP & $\begin{array}{l}R[\mathrm{TH} \cdot], \text { case } 1, \mathrm{MFD}: B_{1 / 2} \approx 200 \mathrm{mT} ; p- \\
\text { I-An: } R(1.8 \mathrm{~T}) \approx-20 \% \text {, triplet mecha- } \\
\text { nism }\end{array}$ & Steiner ${ }^{316}$ Ulrich et al. ${ }^{160}$ \\
\hline${ }^{3} \mathrm{TH}^{+}(6 \mathrm{~S})+p-\mathrm{I}-\mathrm{An}$ & $\mathrm{MeOH}$ & $\mathrm{CP}$ & $\begin{array}{l}R \text { (bleaching quantum yield), MFD, identi- } \\
\text { cal with LFP result }{ }^{160}\end{array}$ & Schlenker and Steiner ${ }^{152}$ \\
\hline${ }^{3} \mathrm{BQ}+$ solvent & 2-PrOH & $\begin{array}{l}\mathrm{CP}, \text { spin- } \\
\text { trapping ESR }\end{array}$ & $\begin{array}{l}R([\text { radicals }]) \approx+16 \%(T=213 \mathrm{~K}, \eta=35 \\
\quad \text { cP })\end{array}$ & Khudyakov et al. ${ }^{139}$ \\
\hline${ }^{3} \mathrm{DQ}+\mathrm{DMAP}$ & glycerol-EtOH & FP & $\begin{array}{l}R(\text { [radicals], case } 2, \mathrm{MFD}, R(120 \mathrm{mT}, 293 \\
\mathrm{K}, 900 \mathrm{cP})=+80 \% \text {, temp } / \text { visc depen- } \\
\text { dence }\end{array}$ & Margulis et al. ${ }^{156}$ \\
\hline${ }^{3} \mathrm{NO}_{2} \mathrm{Ph}-\mathrm{O}-\left(\mathrm{CH}_{2}\right)_{n} \mathrm{NHPh}$ & $\mathrm{ACN}$ & $\begin{array}{l}\text { CP, UV, } \\
\text { HPLC }\end{array}$ & $R\left(\Phi_{\text {disspp }}, 64 \mathrm{mT}\right) \approx-5$ to $-8 \%$ for $n \geq 8$ & Nakagaki et al. ${ }^{317}$ \\
\hline${ }^{3} \mathrm{BQ}+$ solvent & $n$-hexane & TS-LEF & $\begin{array}{l}\text { ransfer Reactions } \\
R([\text { ketyl radicals }]), \text { case } 2: \quad R(0.6 \mathrm{~T}) \approx \\
-8 \%\end{array}$ & Staerk and Razi Naqvi ${ }^{170}$ \\
\hline \multirow{3}{*}{$\begin{array}{l}\text { 1-isoquinolinecarbo- } \\
\text { nitrile* }\left(\mathrm{S}_{1}, \mathrm{~T}_{1}\right)+\text { solvent } \\
\text { 4-methylquinoline-2- } \\
\text { carbonitrile* + solvent }\end{array}$} & EtOH & CP, TLC & $\begin{array}{l}R \text { (yield of cage product) from triplet per- } \\
\quad \text { cursor, case } 2: R(1.8 \mathrm{~T}) \approx+16 \%\end{array}$ & Hata and Yamada ${ }^{316}$ \\
\hline & $\mathrm{EtOH}$ & CP, TLC & $\begin{array}{l}R \text { (yield of cage product) from singlet pre- } \\
\quad \text { cursor, case } 2,3: B_{m}=1.05 \mathrm{~T}, \Delta B \approx 0.2 \\
\mathrm{~T}, R_{\mathrm{M}} \approx-27 \%\end{array}$ & Hata and Hokawa ${ }^{319}$ \\
\hline & $\mathrm{cH}$ & $\mathrm{CP}, \mathrm{TLC}$ & $\begin{array}{l}R \text { (cH substituent product) from triplet } \\
\quad \text { precursor, case } 1,2: R_{\mathrm{M}}=-14 \%, B_{\mathrm{M}} \approx \\
50-80 \mathrm{mT}, R(1.6 \mathrm{~T}) \approx+9 \%\end{array}$ & Hata and Nishida ${ }^{320}$ \\
\hline${ }^{3} \mathrm{AQ} \cdot \mathrm{CO}_{2} \mathrm{C}_{14} \mathrm{H}_{29}(\mathbf{2 2})$ & $\left(\mathrm{CFCl}_{2}\right)_{2}$ & CP, TS-LEF & $\begin{array}{l}R \text { (intramolecular adduct yield), case } 1: \\
\quad \text { triplet biradical } B_{1 / 2} \approx 15 \mathrm{mT}, R(0.26 \\
\text { T) }=-12 \%\end{array}$ & Tanimoto et al. ${ }^{321}$ \\
\hline${ }^{3} \mathrm{AQ}+$ solvent & $\begin{array}{l}\text { glycerol }+ \\
\mathrm{H}_{2} \mathrm{O}\end{array}$ & $\mathrm{CP}$ & $R$ (bleaching rate), $R(125 \mathrm{~m} \mathrm{~T}) \approx+40 \%$ & Margulis et al. ${ }^{156}$ \\
\hline${ }^{3} \mathrm{FMN}+$ solvent & $\begin{array}{l}\text { glycerol }+ \\
\text { EtOH }\end{array}$ & FP & $\begin{array}{l}R \text { (radical yield), case } 2: R(125 \mathrm{mT}) \approx \\
\quad+19 \% \\
R(2 \text { nd-order radical decay constant), case } \\
\quad 1: B_{\mathrm{s}}=50 \mathrm{mT}, R_{\mathrm{s}}=-8 \%\end{array}$ & \\
\hline
\end{tabular}


TABLE 8 (Continued)

\begin{tabular}{|c|c|c|c|c|}
\hline reactants $^{a}$ & solvent ${ }^{b}$ & $\begin{array}{l}\text { method of } \\
\text { observation }^{c}\end{array}$ & characteristics of $\mathrm{MFE}^{d}$ & ref \\
\hline $\begin{array}{l}{ }^{3} \mathrm{DQ}+\text { solvent } \\
{ }^{3} \mathrm{BQ}+\text { solvent } \\
{ }^{3} \mathrm{XO}-n \cdot \mathrm{XH}(\mathbf{2 4} n)\end{array}$ & $\begin{array}{l}\text { glycerol + EtOH } \\
\text { glycerol + EtOH } \\
\text { ACN }\end{array}$ & $\begin{array}{l}\text { FP } \\
\text { FP } \\
\text { LFP }\end{array}$ & $\begin{array}{l}R(\text { radical yield }), \text { case } 2: R(125 \mathrm{mT}) \approx+10 \% \\
R(\text { radical yield }), \text { case } 2: R(125 \mathrm{mT}) \approx+12 \% \\
\tau_{\mathrm{RP}}(0.8 \mathrm{~T}) / \tau_{\mathrm{RP}}(0 \mathrm{~T})=2.8,4.7,7.9,7.9,16.9,15.0 \\
\quad 19.1(n=2,3,4,5,6,8,12)\end{array}$ & Margulis et al. ${ }^{156}$ \\
\hline $\begin{array}{l}\text { dibenzoyl peroxide } \\
\text { ( } \mathrm{S}_{1} \text { sens) }\end{array}$ & toluene & VPC & $\begin{array}{l}\text { molytic Bond Cleavage } \\
R \text { (yield of cage product), } R(1 \mathrm{~T}) \approx-4 \%, R(4 \mathrm{~T}) \approx \\
-8 \% \Delta g \mathrm{RP} \text { mechanism } \\
R \text { (various product yields), case } 2, \text { case } 1,2\end{array}$ & Tanimoto et al. ${ }^{130}$ \\
\hline${ }^{3}$ di-tert-butyl ketone & $n$-decane & GLC & $\begin{array}{l}R\left(\text { cage product pivaldehyde), case } 1: B_{1 / 2} \approx 10 \mathrm{mT} \text {, }\right. \\
\quad R_{\mathrm{s}} \approx-17 \%\end{array}$ & Fischer ${ }^{323}$ \\
\hline${ }^{3}$ alicyclic ketones $(26 n)$ & $\mathrm{MeOH}$ & LFP & $\begin{array}{l}R \text { (biradical decay constant), case } 1,3: B_{\mathrm{M}} \text { variable } \\
\quad \text { with } n, R_{\mathrm{M}}=+13 \%, R_{\mathrm{s}}>-16 \%\end{array}$ & Zimmt et al. ${ }^{324}$ \\
\hline \multicolumn{5}{|c|}{ Miscellaneous Reaction Types } \\
\hline $\begin{array}{l}\text { trans-olefins, }{ }^{3} \text { sens } \\
\quad \text { trans } \rightarrow \text { cis isomer }\end{array}$ & & & results of ref 326 could not be reproduced & $\begin{array}{l}\text { Leshina et al.. }{ }^{310} \text { Hayashi } \\
\text { and Nagakura }{ }^{325}\end{array}$ \\
\hline isoquinoline $N$-oxide (11) & alcohols & TLC, CP & $\begin{array}{l}R \text { (lactam isomer yield), case } 3: B_{\mathrm{M}} \geq 0.7 \mathrm{~T} \\
\quad \text { solvent dependent, } R_{\mathrm{M}} \leq-20 \%\end{array}$ & $\begin{array}{l}\text { Hata, }{ }^{33,134,327} \\
\text { Hata et al. }{ }^{135}\end{array}$ \\
\hline $\begin{array}{l}{ }^{3} \text { phthalocyanines } \\
\left(\mathrm{Al}^{\mathrm{III}}, \mathrm{Si}^{\mathrm{IV}}, 2 \mathrm{H}^{+}\right)\end{array}$ & $\mathrm{EtOH}, \mathrm{cH}$ & LFP & $\begin{array}{l}R\left(1 \text { st- and } 2 \text { nd-order } T_{1} \text {-decay constant), } R(7 \mathrm{~T}\right. \\
\left.\quad k_{1}\right)=-37 \% \text { to }-71 \%, R\left(7 \mathrm{~T}, k_{2}\right)=+9 \% \text { to }+23 \%\end{array}$ & Frink et al. ${ }^{116}$ \\
\hline $\begin{array}{l}{\left[\mathrm{Ru}\left(\mathrm{NH}_{3}\right)_{5} \mathrm{X}\right]^{2+}} \\
\quad(\mathrm{X}=\mathrm{Cl}, \mathrm{Br})\end{array}$ & $\mathrm{H}_{2} \mathrm{O}$ & PSFP & $\begin{array}{l}\mathrm{NH}_{3} \text { and } \mathrm{X}^{-} \text {photoaquation, } R\left(\Phi_{\mathrm{x}}^{-}, 2.4 \mathrm{~T}\right)= \\
\quad+(30-40) \%, R\left(\Phi_{\mathrm{NH}_{9}}, 2.4 \mathrm{~T}\right) \approx-10 \%\end{array}$ & Ferraudi and Pacheco ${ }^{772}$ \\
\hline $\mathrm{K}_{2}\left[\mathrm{Co}(\mathrm{CN})_{6}\right]$ & $\mathrm{H}_{2} \mathrm{O}$ & PSFP & $\mathrm{CN}^{-}$photoaquation, $B_{\mathrm{s}} \approx 1.8 \mathrm{~T}, R_{\mathrm{s}}\left(\Phi_{\mathrm{CN}^{-}}\right) \approx 100 \%$ & Ferraudi et al. ${ }^{773}$ \\
\hline \multicolumn{5}{|c|}{ 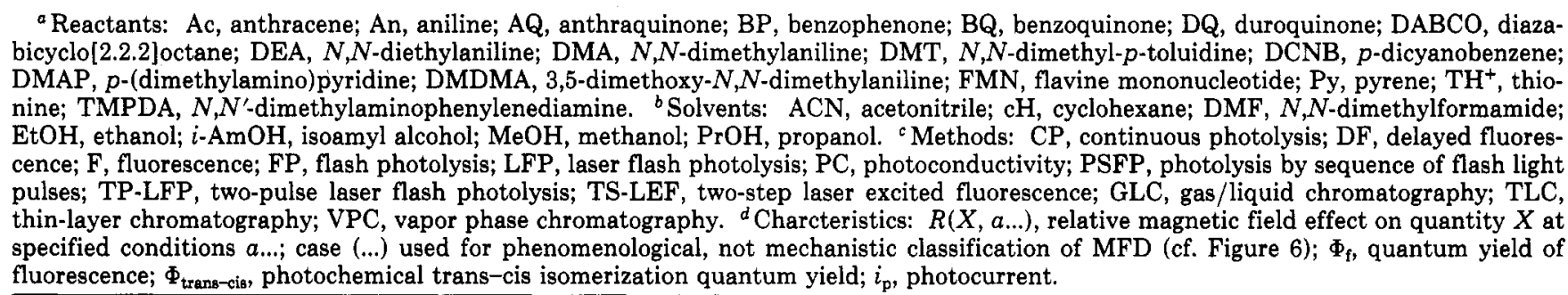 } \\
\hline
\end{tabular}

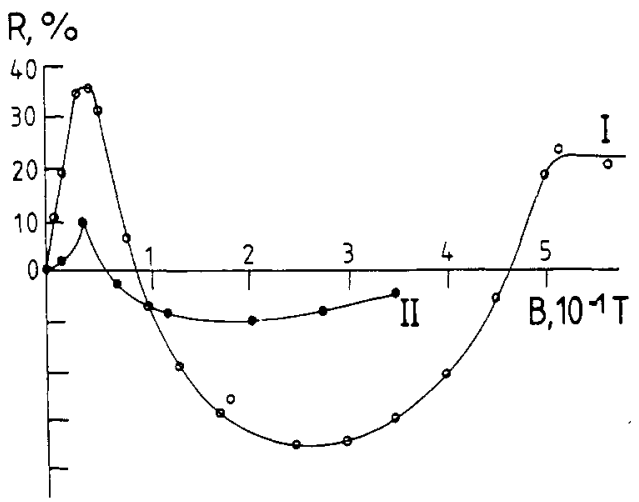

Figure 13. Relative MFE on the initial rate of $\mathrm{N}_{2}$ evolution in the reactions of $\mathrm{C}_{6} \mathrm{H}_{5} \mathrm{~N}_{2}^{+} \mathrm{BF}_{4}^{-}$with $\mathrm{FeSO}_{4}$ (I) and $p$ $\mathrm{CH}_{3} \mathrm{C}_{6} \mathrm{H}_{5} \mathrm{~N}_{2}{ }^{+} \mathrm{BF}_{4}^{-}$with $\mathrm{FeSO}_{4}$ (II) in aqueous DMSO (1:1). Reprinted from ref 298 with permission of T. G. Samarskaya, I. P. Gragerov, and L. A. Kiprianova; copyright 1985 Plenum Publishing $\mathrm{Co}$.

A MFE on the thermolysis of dilauroyl peroxide in octane (eq 41) has been found by Tanimoto et al..$^{296} \mathrm{~A}$ fairly weak effect (case 1 type, $R_{\mathrm{S}}^{\mathrm{c}}=+3-6 \%, B_{\mathrm{S}} \approx 10$ $\mathrm{mT}$ ) appeared in the yield of the cage product $\mathrm{C}_{22} \mathrm{H}_{46}$. It has been attributed to the hfc mechanism.

$$
\left.\mathrm{C}_{11} \mathrm{H}_{23} \mathrm{CO}_{2}\right)_{2} \rightarrow{ }^{1} \overline{\mathrm{C}_{11} \mathrm{H}_{23} \cdot{ }^{\circ} \mathrm{C}_{11} \mathrm{H}_{23}} \underbrace{\text { Cage }}_{\text {escape }} \mathrm{C}_{11} \mathrm{H}_{22}, \mathrm{C}_{11} \mathrm{H}_{24}
$$

While some older reports on MFEs for reactions involving paramagnetic metal ions ${ }^{1}$ have not been subjected to a critical mechanistic reinvestigation, there are several recent reports on MFEs in redox reactions involving transition-metal ions.

Molin et al. ${ }^{297}$ investigated the MFD of the rate of $\mathrm{H}_{2} \mathrm{O}_{2}$ decomposition, catalyzed by an $\mathrm{Fe}^{\mathrm{III}}$ dimer complex with EDTA. The effect is of the case 2 type $(R(0.8$ $\mathrm{T})=+20 \%$ ) and is attributed to the $\Delta g$ mechanism inducing ISC in $\mathrm{Fe}^{3+}-\mathrm{O}_{2}^{-}$paramagnetic pairs.

Samarskaya et al. ${ }^{298}$ recently reported on very marked MFEs (cf. Figure 13) on the rate of reduction of phenyldiazonium and $p$-tolyldiazonium borofluorides by $\mathrm{Fe}^{2+}$ ions (eq 42).

$$
\begin{aligned}
& \mathrm{ArN}_{2}{ }^{+}+{ }^{5} \mathrm{Fe}^{2+}={ }^{5} \overline{\mathrm{ArN}_{2} \mathrm{Fe}^{3+}}-\mathrm{ArN}_{2}{ }^{0}+{ }^{6} \mathrm{Fe}^{3+} \\
& -\mathrm{N}_{2} \\
& \mathrm{Ar}^{\circ}-\mathrm{ArH}
\end{aligned}
$$

Assuming high-spin configuration for the $\mathrm{Fe}^{\mathrm{II} / \mathrm{III}}$ ions, the initial encounter pair would be of quintet multiplicity whereas the geminate pair, after its formation, might undergo a multiplicity change from quintet to septet, rendering recombination spin forbidden. It has been argued that the $h f c$ and $\Delta g$ mechanisms should be responsible for the MFD observed. One should expect, however, that, as with triplets in liquid solution (cf. section V.D) spin relaxation due to rotational diffusion of the $\left[\mathrm{Fe}^{\mathrm{II}}\left(\mathrm{H}_{2} \mathrm{O}\right)_{6}\right]^{3+} \mathrm{ZFS}$-tensor axes might be of major importance for such a system.

Fairly large MFEs of the case 1,2 type have been found by Perito and Corden, ${ }^{301}$ who investigated the rates of catalytic thermal oxidation of 2,6-di-tert-butylphenol with molecular oxygen. Catalysts used were 
$\mathrm{Co}(\mathrm{SMPDT})$ and $\mathrm{Mn}\left(5-\mathrm{NO}_{2} \mathrm{SMPDT}\right)$ complexes (SMPDT $=$ bis $((3$-salicylideneamino)propyl)methylamine). The MFE data are $R_{\mathrm{m}} \approx+50 \%(+80 \%), B_{\mathrm{m}}$ $\approx 0.1 \mathrm{~T}(0.2 \mathrm{~T})$, and $B_{\mathrm{c}} \approx 4 \mathrm{~T}(0.7 \mathrm{~T})$ for the $\mathrm{Co}(\mathrm{Mn})$ complex. Magnetically sensitive steps of the reaction mechanisms have been suggested, but not yet definitely assigned.

There have been some attempts to see MFEs on the self-oscillatory Belousov/Zhabotinsky reaction, in which malonic acid is oxidized by $\mathrm{KBrO}_{3}$ with the $\mathrm{Ce}^{3+} / \mathrm{Ce}^{4+}$ redox couple as a catalyst. Whereas Broomhead and McLauchlan ${ }^{299}$ reported the absence of a MFE in this reaction, Agulova and Opalinskaya ${ }^{300}$ reported an effect of $\leq 14 \%$ on the amplitudes of the oscillations and the width of their statistical scatter. Astonishingly enough, the effects have been found at very low magnetic field strength of $0.0025-0.1 \mathrm{mT}$ (i.e., between zero field and the earth's magnetic field).

More examples of MFEs in thermal reactions may be found in sections IV.C.3 (luminescence in liquid solutions), IV.E (interfaces), IV.F (biological systems), and IV.H (magnetic isotope effects).

\section{Photochemical Reactions}

From the energetic point of view photochemical reactions have a great advantage over thermal reactions in producing species with unpaired electron spins, which is necessary for any mechanism whereby magnetic field effects on chemical kinetics may ensue. Radical pairs are of special importance in that respect. They can be formed in three main groups of chemical reactions: by electron transfer, $\mathrm{H}$-atom transfer, and homolytic bond cleavage reactions. The examples of magnetic field effects on photochemical reactions in homogeneous solutions listed in Table 8 are grouped in this way. It is conspicuous that, although the three types of reactions mentioned would allow for a much broader chemical variety of examples, the investigations tend to concentrate on a rather restricted number of favorite chemical systems. This indicates that research on chemical magnetic field effects is still concentrated in the hands of research groups studying primarily the fundamental features of these effects rather than applying them as a standard tool in mechanistic photochemistry.

(a) Electron-Transfer Reactions with Excited Singlet States. Studies in this field have been mainly concentrated on the excited singlets of pyrene and anthracene. In almost all cases these have been used as electron acceptors with aromatic amines as electron donors. An exception to this situation is the reaction of pyrene with dicyanobenzene, ${ }^{306,765}$ where the excited singlet aromatic is the electron donor.

The relevant reaction steps necessary to describe the magnetic field effects are schematically shown in Figure 14. Photoelectron transfer with an excited singlet may involve the intermediacy of a singlet exciplex ${ }^{1}(\mathrm{AD})^{*}$, dissociating reversibly into a singlet radical ion pair, which may be also formed in a direct process in solvents of high polarity. This geminate radical pair may recombine to the singlet ground state, separate further to free radicals, or undergo a multiplicity change to the corresponding triplet radical ion pair, which can either separate to the free radicals or else recombine to yield an excited triplet species if energetically feasible. ${ }^{765}$ In

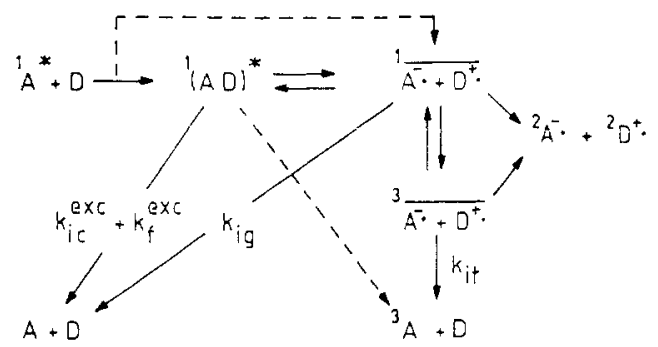

Figure 14. Scheme of primary reaction steps following photoelectron transfer with excited singlets. The rate constants are designated after Weller. ${ }^{304}$

cases where dissociation of the exciplex is not very fast, other channels of deactivation may become important. These are internal conversion and fluorescence leading to the ground state or intersystem crossing leading to a locally excited triplet state. The MFE influencing directly the rate of multiplicity conversion of the radical pair may affect several channels: formation of free radicals, locally excited triplet states, and exciplex fluorescence. It should be also detectable via the repopulation kinetics of the ground-state molecules. Evidence for the triplet recombination channel has also come from CIDNP experiments. ${ }^{329}$

The first observations of MFEs on the so-called fast triplet formation in fluorescence quenching of pyrene by diethylaniline (DEA) were reported in 1976 by the groups of Weller ${ }^{302}$ and Michel-Beyerle. ${ }^{157}$ These papers have found adequate attention in various reviews. ${ }^{31,45,52,328}$ In the experiments fast triplet formation was directly observed by laser flash photolysis or its two-pulse modification. Furthermore, the magnetic field dependence was monitored by stationary delayed fluorescence, probing the magnetic field dependence of the overall triplet concentration. The results provided unequivocal evidence that fast triplet formation in this system was due to a magnetic-field-dependent change of multiplicity in the geminate singlet radical pair. However, in methanol as a solvent there is also a contribution to fast triplet formation from intersystem crossing in the intermediate exciplex, which is due to spin-orbit coupling and is not magnetic field sensitive. $^{302}$ Hence the MFE provides a unique means to separate the contributions to fast triplet formation from the exciplex and from the geminate radical pair.

In these systems, where radical ion pairs with Coulombic attraction are formed, the dissociative lifetimes of the exciplex and the radical pair are extremely solvent polarity dependent. A detailed study of this solvent dependence using the magnetic field effect on the triplet yield was reported by Werner et al. ${ }^{303}$ for the systems pyrene/DMA and pyrene/DMDMA (cf. Table 8). It was found that as the solvent was changed from the less polar solvent ethanol to the more polar solvent acetonitrile, the magnetic field effect on the triplet yield increased because exciplex dissociation is more efficient in the more polar solvent and its contribution to fast triplet formation is reduced. From the experimental data a detailed analysis of the various rate constants in Figure 14, including their solvent dependence, could be given. ${ }^{304}$ This work demonstrates the useful contribution of MFE investigations to the exploration of mechanistic details in electron-transfer reactions.

Other experiments along this line, however monitoring magnetic-field-dependent photoconductivity, 


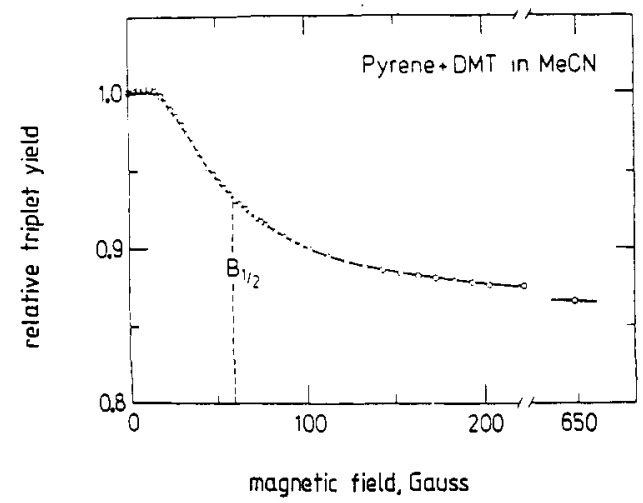

Figure 15. MFD of pyrene triplet yield produced by fluorescence quenching of pyrene by $N, N$-dimethyl-p-toluidine in acetonitrile. The triplet yield was derived from delayed fluorescence measurements. Reprinted from ref 306 with kind permission of A. Weller; copyright 1983 Elsevier Science Publishers B.V.

were performed by Frankevich's group. They were able to demonstrate that a sign inversion of the MFE on the yield of free ion radicals occurs when going from acetonitrile $(\mathrm{DK}=38)$ to isopropyl alcohol $(\mathrm{DK}=18){ }^{762}$ This effect, which was also observed in solvent mixtures, ${ }^{763}$ was attributed to the occurrence of singlet exciplex formation from the radical ion pair in the less polar solvent, so that in this case the lifetime of the singlet radical ion pair becomes shorter than that of its triplet counterpart, whereas in acetonitrile, where the singlet exciplex is less easily accessible, the opposite is true.

The reversibility of fluorescent exciplex formation from geminate radical pairs has been demonstrated more directly by MFEs on the exciplex fluorescence quantum yield (cf. section 3). These effects may be particularly pronounced if donor and acceptor radical are linked together by a mobile molecular chain. Since in such a case the magnetic-field-independent dissociation channel is closed, the magnetic field dependence of the radical pair lifetime becomes most pronounced (vide infra). The MFD of the triplet yields (MARY spectra) in the numerous investigations by the groups of Weller and Michel-Beyerle is generally of case 1 type. The effects are governed by the magnetic field modulation of the hyperfine coupling mechanism, since the range of magnetic fields was generally not extended above $0.1 \mathrm{~T}$ and the $\Delta g$ mechanism is of no importance here. It has been found that the typical value of $B_{1 / 2}$ (cf. Figure 15) depends in a characteristic way on the hyperfine coupling constants of the magnetic nuclei and on the lifetime of the radical pairs. It is, however, not always the chemical lifetime but rather the effective electron spin correlation time that is of importance. The latter may be shorter than the chemical lifetime in the case of electron hopping between radicals and diamagnetic molecules or in the case of early-time probing of the MFE, i.e., by the time delay in timeresolved measurements. Only if all of these lifetimedetermining processes (chemical, electron hopping, and probing) are sufficiently slow (usually longer than 20 $\mathrm{ns}$ ) is the $B_{1 / 2}$ value indicative of the effective hyperfine coupling in the radical pair. This was demonstrated by the occurrence of a $\mathrm{H} / \mathrm{D}$ magnetic isotope effect on the $B_{1 / 2}$ value. ${ }^{112,303,306,314}$ A quantitative correlation was established by Weller et al. ${ }^{306}$ using a series of 16 different combinations of pyrene or pyrene- $d_{10}$ with var-

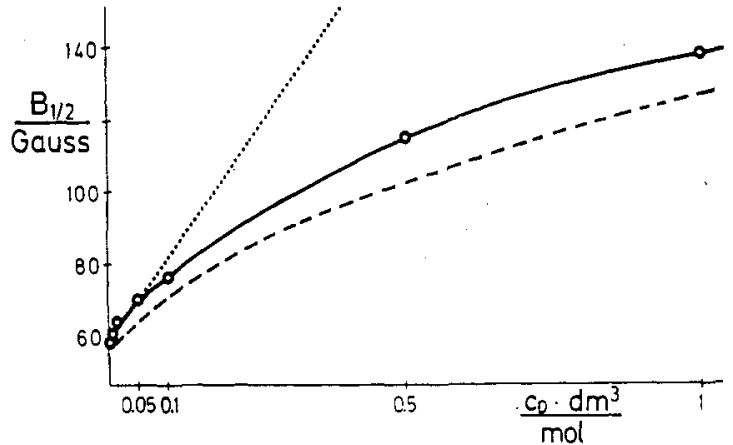

Figure 16. $B_{1 / 2}$ values of MFE on pyrene triplet formation in fluorescence quenching by $N, N$-dimethylaniline in methanol. Dependence on donor concentration $C_{D}(--)$ Theoretical results according to ref 651 ; (...) Heisenberg energy broadening effect corresponding to electron hopping between donor molecules. Reprinted from ref 307 with kind permission of A. Weller; copyright 1983 Elsevier Science Publishers B.V.

ious aromatic amines, with some of their D- or F-substituted derivatives, or with dicyanobenzene:

$$
B_{1 / 2}=2\left(B_{1}^{2}+B_{2}^{2}\right) /\left(B_{1}+B_{2}\right)
$$

The individual $B_{i}$ values characterizing the radicals ( $i$ $=1,2)$ are given by

$$
B_{i}=\left(\sum_{j} I_{i j}\left(I_{i j}+1\right) a_{i j}^{2}\right)^{1 / 2}
$$

Equation 43 gives numerical results similar to those from an expression derived by Schulten ${ }^{330}$

$$
\left[1-1 / 3\left(B_{1} / B_{1 / 2}\right)^{2}\right]\left[1-1 / 3\left(B_{2} / B_{1 / 2}\right)^{2}\right]=0.9
$$

from a semiclassical consideration of electron spin motion. From the agreement of experimental $B_{1 / 2}$ values with eq 43 or 44 one may conclude that the lifetimes of the radical pairs investigated are longer than $\tau=\hbar / B_{1 / 2}$.

Experimentally, it was first shown by Michel-Beyerle et al. ${ }^{312}$ with the anthracene $\left(\mathrm{S}_{1}\right)$ /DMA system in acetonitrile that $B_{1 / 2}$ increases as the delay time of the probing laser pulse is decreased. Thus for delay times of 15,7 , and $5 \mathrm{~ns}, B_{1 / 2}$ values of $5.1,5.8$, and $6.9 \mathrm{mT}$ were observed, respectively.

This effect has been reproduced theoretically. ${ }^{312}$ Its obvious interpretation follows from inspecting a diagram showing the spin evolution of a radical pair in various magnetic fields (cf. Figure 46). MFEs are first seen at longer times, and the crossover from the zerofield curve to the limiting high-field curve occurs at successively shorter times as the magnetic field is increased. The analytical formula provided by Haberkorn $^{331}$ for the short-time behavior (cf. section V.C.3) describes this feature quite nicely. The phenomenon may be also conceived as a lifetime-broadening effect. Due to the short delay time of observation, the energy levels are broadened and higher magnetic fields are necessary to provide a Zeeman splitting sufficient for separating the individual levels. Such lifetime-broadening effects have been also observed by Weller's group. 307

Similar effects on $B_{1 / 2}$ are observed when the donor concentration is increased, so that electron hopping between diamagnetic donor molecules and donor radicals may occur during the lifetime of a geminate pair. ${ }^{305,307,313,314}$

In Figure 16 is shown the increase of the $B_{1 / 2}$ value 
as the DMA concentration for pyrene singlet quenching in methanol is increased. The straight line, describing the initial slope of the $B_{1 / 2}$ versus concentration of DMA curve, corresponds to the lifetime-broadening effect; the deviation from this slope at higher DMA concentrations has to be explained by an exchange narrowing, which comes into play if many hopping processes during the radical pair lifetime wipe out the effect of the donor hyperfine coupling. In the limiting case, which has not yet been approached experimentally, one should expect that the $B_{1 / 2}$ value is even lower than at low donor concentrations because then it is only determined by the hyperfine coupling in the acceptor radical. It has also been noted that the effect of probing pulse delay time on $B_{1 / 2}$ can be suppressed if the lifetime broadening by the hopping process is strong enough. ${ }^{307}$

It is of interest to note that electron-hopping effects between donor radicals are also demonstrated by unusual CIDNP patterns obtained in the pyrene singlet/ diethylaniline system with high donor concentrations. ${ }^{332}$

A mechanism similar to that in the singlet radical ion pairs mentioned above, with a more efficient recombination into the triplet channel, has been invoked by Tanimoto et al. ${ }^{172}$ to account for the MFE shown by the photoconductivity of tetramethyl-p-phenylenediamine (TMPDA) photoionized by the action of $308-\mathrm{nm}$ laser pulses. It was assumed that the $e^{-} /$TMPDA $^{*+}$ pair produced on photoionization may dissociate to yield free charges, detectable by photoconductivity, a process that has to compete with singlet/triplet spin evolution and successive pair recombination to yield the TMPDA triplet state. Since triplet pairs are formed less efficiently in magnetic fields, this is favorable for the yield of dissociated charge carriers. The fairly high $B_{1 / 2}$ value of $0.13 \mathrm{~T}$ indicates that electron spin relaxation may significantly contribute to the radical pair multiplicity change.

The singlet-triplet multiplicity change in radical ion pairs produced by photoelectron transfer between stilbenes and singlet excited pyrene has been used by Leshina et al ${ }^{310}$ for indirect sensitization of stilbene trans-cis isomerization via the triplet channel. Rather strong MFEs of about $-30 \%$ have been observed, but the MFD shows significant differences when stilbene and its perfluorinated analogue are compared. Whereas the former saturates at about $20 \mathrm{mT}$, the corresponding behavior of the perfluorinated compound extends to about $0.1 \mathrm{~T}$ and turns to a case 2 behavior ( $\Delta g$ mechanism) at higher fields. Theoretically, the MFD curves are well reproduced on the basis of the semiclassical model of spin motion. Furthermore, CIDNP effects have corroborated the mechanism. ${ }^{311}$

In connection with sensitized cis-trans isomerization of olefins, it should be mentioned that an early report by Gupta and Hammond ${ }^{326}$ on MFEs in triplet-benzophenone-sensitized stilbene isomerization has not been confirmed by other groups. ${ }^{310,325}$

(b) Electron-Transfer Reactions with Excited Triplet States. When radical pairs are produced by electron-transfer reactions with excited triplet states, their energy is usually too low to repopulate the locally excited triplet state. Thus the only possibility for recombination is to the singlet ground state. Since this process is spin forbidden for a triplet radical pair, the escape reaction usually dominates, so that free radical yields are generally high in triplet electron-transfer reactions..$^{333,334}$ Geminate recombination requires triplet-singlet transitions in the radical pairs, which are sensitive to magnetic fields. Therefore the free radical yield, too, should exhibit MFEs. It should be noted, however, that if radical escape is the predominant channel, its magnetic field modulation by the magnetic-field-dependent recombination process is generally small. On the other hand, probing the yield of geminate recombination to the ground state is a more difficult experimental task, since usually a large background of ground-state molecules not taking part in the photoprocess is present. Thus, in order to create favorable conditions for observing MFEs on the free radical yield, the efficiency of the escape channel has to be decreased, which is usually done by lowering the temperature or by using highly viscous solvents.

The first effects of this type were observed by Periasamy and Linschitz ${ }^{315}$ for the electron-transfer reaction between $\mathrm{DABCO}$ and fluorenone triplet in propylene carbonate. Their results, which were obtained over a wide temperature range, illustrate the arguments given above: whereas at $343 \mathrm{~K}$ and $1 \mathrm{cP}$ with a zerofield radical yield of 0.89 the maximum MFE was only $1.6 \%$, at $223 \mathrm{~K}$ and $45.3 \mathrm{cP}$ with an absolute radical yield of only 0.13 the MFE amounted to $24 \%$. The MFE was saturated at fields above $27 \mathrm{mT}$ and is of the case 1 type, indicating the influence of the hyperfine coupling mechanism.

High-solvent-viscosity and low-temperature effects have also been successfully applied by Kuzmin and co-workers ${ }^{139,156}$ to observe MFEs on the radical yield from triplet electron-transfer reactions. Radicals produced in a reaction between benzoquinone triplet and 2-propanol were spin-trapped and the adducts quantitatively assessed by ESR spectroscopy. Radicals produced in the reaction between duroquinone triplet and $p$-(dimethylamino)pyridine in glycerol/ethanol mixtures have been detected by conventional flash photolysis. The latter system yields rather high MFEs of up to $80 \%$ that are of the case 2 MFD type. Hyperfine coupling and relaxation mechanism contributions are invoked to account for this.

MFEs on the free radical yield from triplet electron-transfer reactions in low-viscosity methanolic solutions at room temperature have been found by Steiner and co-workers ${ }^{152,160,316}$ for the reaction between thionine triplet (6S) and various monohalogen anilines. Laser

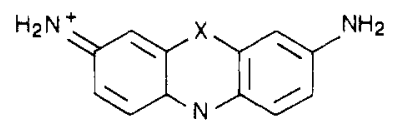

$6 x(x=0.5)$

flash photolysis and steady-state photokinetics yielded quantitatively identical MFD curves for the production of semireduced thionine radicals and the permanent photobleaching quantum yield of the dye. As shown in Figure 17 the radical yield is decreased by a magnetic field, the MFE being enhanced by heavy-atom substitution of the electron donor. The MFD curves show saturation above $1 \mathrm{~T}$, with $B_{1 / 2}$ values typically on the order of $0.2 \mathrm{~T}$.

A first explanation of these effects was suggested in terms of the radical pair $\Delta g$ mechanism. ${ }^{316}$ However, an application of more quantitative criteria, as follow from a theoretical calculation by Schulten and Epstein ${ }^{68}$ on the $\Delta g$ mechanism in triplet radical pairs without 


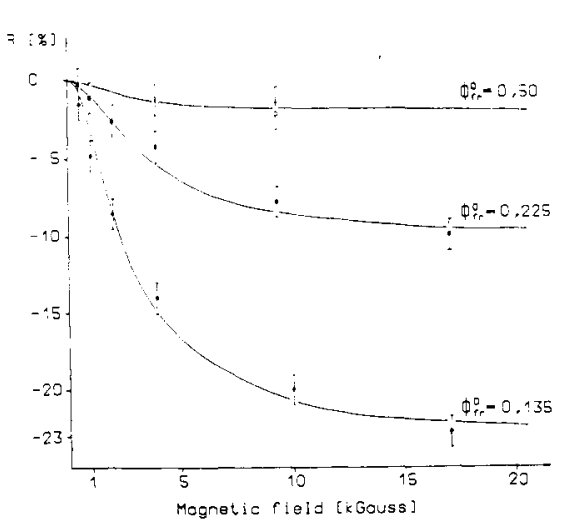

Figure 17. Relative MFE $(R)$ on radical yield from triplet quenching of thionine by halogen anilines in methanol: ( $p$-iodoaniline; (a) $o$-iodoaniline; $(\diamond) m$-iodoaniline; (a) $p$ bromoaniline. $\Phi_{\mathrm{fr}}{ }^{0}$ values given are absolute quantum yields of radical formation in zero field. The solid lines are theoretical fits according to the triplet mechanism (adapted from ref 160 ).

Coulombic attraction, the effects shown in Figure 17 are much larger and saturate at much lower fields than are expected for the $\Delta g$ mechanism. A quantitative interpretation was then developed in terms of the triplet mechanism, ${ }^{80,335}$ which comes into play through the intermediacy of a triplet exciplex formed as a primary product in the electron-transfer reaction. ${ }^{336}$

It was shown that in a sandwich-type exciplex between electron donor and electron acceptor spin-orbit coupling at the donor-localized heavy-atom substituent will be much less efficient in that particular triplet substate where the electron spin is oriented parallel to the aromatic plane of the donor. ${ }^{80}$ Thus the requirements for the triplet mechanism, as described in section II, are met. From a quantitative analysis of the MFD curves decay rate constants of several triplet exciplexes could be determined. Thus, e.g., for the thionine $/ p$ iodoaniline triplet exciplex rate constants of $6.8 \times 10^{10}$ and $5 \times 10^{9} \mathrm{~s}^{-1}$ were obtained for ISC $\left(\mathrm{T} \rightarrow \mathrm{S}_{0}\right.$ ) and radical formation, respectively.

(c) Photochemical Hydrogen-Atom-Transfer Reactions. Hydrogen-atom abstractions are characteristic of the photochemistry of $n \pi^{*}$ triplet states as may be typically found in carbonyl compounds or azaheteroaromatics. The primary product of such reactions is a neutral triplet radical pair. In this case diffusive separation of the radical pairs is much more efficient than with radical ion pairs, and it is generally hard to detect MFEs from escape product yields unless the solvent cage effect is increased by some auxiliary means such as micellar solubilization (vide infra) or binding of the radicals to other aggregates as was the case with the thermal reactions of lithium alkyls.

The first MFE on free radical yields from photochemical hydrogen atom transfer was reported by Staerk and Razi Naqvi ${ }^{170}$ for the reaction of benzophenone triplet with the solvent $n$-hexane. A rather weak decrease of the free radical yield with a case 2 magnetic field dependence was reported. It was suggested that the MFE was due to the $\Delta g$ mechanism. Theoretical analysis of the system, however, by Schulten and Epstein ${ }^{68}$ revealed that, with reasonable assumptions on the $\Delta g$ value and on the diffusional motion, the MFD predicted theoretically was greatly at variance with the experimental observation. It was $\operatorname{argued}^{68}$ that other magnetic-field-dependent processes (e.g., $\mathrm{T}+\mathrm{D}$ energy transfer) might be responsible for the effects observed.

The MFD of various photochemical hydrogen-atom abstractions was studied by Margulis et al. ${ }^{156}$ These authors used solvent mixtures of water or ethanol with glycerol to obtain very highly viscous solvents. Varying the temperature and the solvent composition, they showed that the MFEs are determined by the value of $T / \eta$. MFEs on the free radical yield (determined by flash spectroscopy) or on the permanent bleaching reaction (determined by continuous photolysis) became detectable only at $T / \eta$ values smaller than $10 \mathrm{~K} / \mathrm{cP}$. At room temperature this corresponds to viscosities larger than $20 \mathrm{cP}$. In the systems investigated by Margulis et al. ${ }^{156}$ (cf. Table 8). The magnetic field causes an increase of free radical yield, which results from a suppression of triplet-singlet transitions by a magnetic field. The MFD curves are of the case 2 type, which is taken as evidence for a contribution of the relaxation mechanism. Remarkably, Margulis et al. also found MFEs on the second-order recombination rate constant in the case of riboflavin semiquinone radicals and benzophenone ketyl radicals. The second-order bulk recombination rate is slowed down by the magnetic field, demonstrating that $\mathrm{F}$ pairs behave rather like geminate triplet pairs, which has also been confirmed in many CIDNP investigations.

MFEs in the photochemistry of quinoline and isoquinoline derivatives have been reported by Hata et al. ${ }^{318-320}$ Photochemical hydrogen abstractions by the ring nitrogen from the solvent ethanol are believed to be the primary reactions in the photoreactions of 1 isoquinolinecarbonitrile (7) and 4-methylquinoline-2carbonitrile (9). For the reaction of 7 (eq 46) the
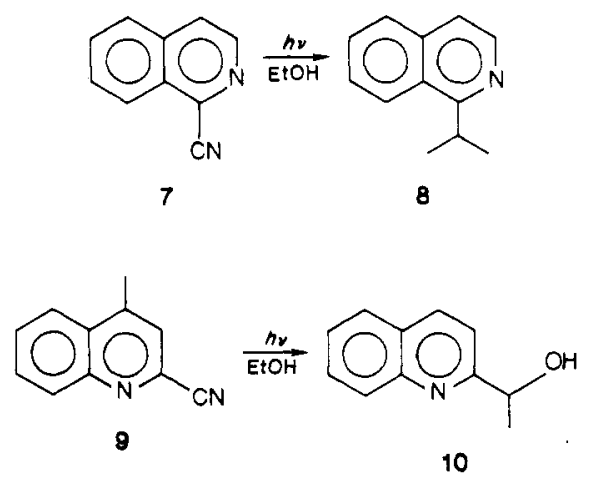

product yield MFD is of the case 2 type. It is assumed that a triplet radical pair is the primary product originating from an excited triplet state, and the $\Delta g$ mechanism is invoked to explain the MFD curves.

For compound 9 the MFD of the yield of product 10 is much more complicated (case 2,3) ${ }^{319}$ It is explained as a superposition of the contributions of triplet radical pairs, their recombination being dominated by the $\Delta g$ mechanism, and of singlet radical pairs of somewhat different structure with a larger exchange interaction, so that $\mathrm{S}-\mathrm{T}_{+}$level crossing occurs at higher fields. 'The MFD characteristics are quite sensitive to the solvent. ${ }^{320}$ Although we believe that the interpretation of these effects requires a more rigorous theoretical and mechanistic analysis, the results suggest that MFEs might be more generally exploited by photochemists for obtaining additional mechanistic information. 


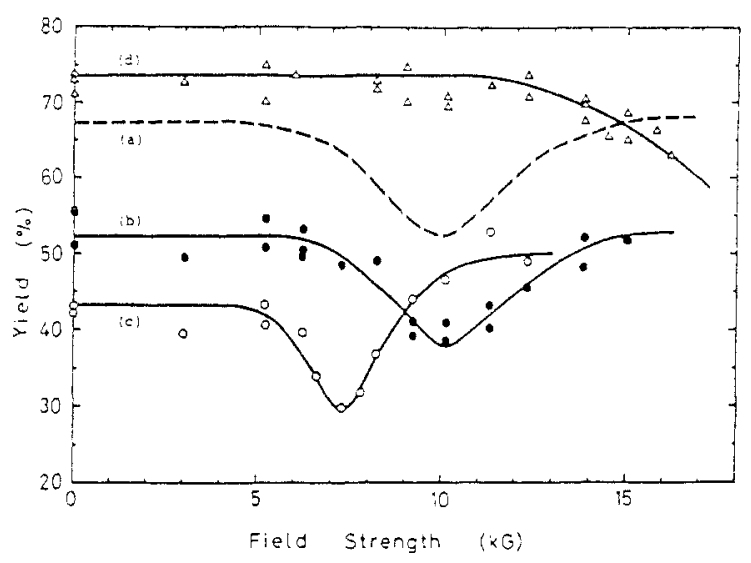

Figure 18. Chemical yield of lactam 12 in reaction 48 as a function of magnetic field strength. Solvents: (a) ethanol; (b) 2-propanol; (c) tert-butyl alcohol; (d) methanol. Reprinted from ref 135 with kind permission of N. Hata; copyright 1979 Japan Publications Trading Co. (ref no. CY-RT 88012).

Another interesting example is the photoisomerization of isoquinoline $N$-oxide (11). ${ }^{133-135,327}$ Only the

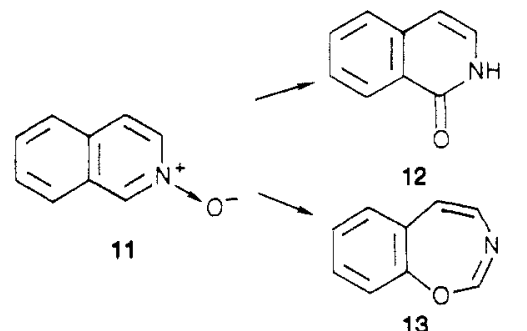

yield of the lactam 12 is magnetic field dependent. The effect is of case 3 type and appears only in hydroxylic solvents. The $B_{\max }$ value depends on the type of alcohol used. It moves to higher fields as the $\mathrm{p} K_{\mathrm{a}}$ of the alcohol decreases (cf. Figure 18). The effects are suggested to be due to the level-crossing mechanism in a singlet radical pair of structure 14 , which is assumed to mediate

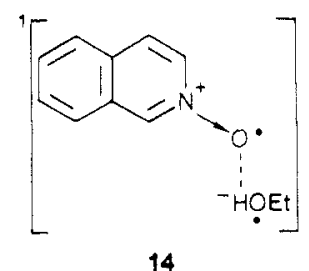

an excited-state proton transfer, furthering formation of the lactam isomerization product. A singlet/triplet transition in $\mathbf{1 4}$ would favor radical pair dissociation, which is thought to lead finally to preferential regeneration of the starting material. The mechanism must certainly be regarded as rather speculative. The findings borne out in Figure 18 are, however, unique in the field of photochemical MFEs in that they represent the only examples of apparently pure case 3 MFD curves, corresponding to fairly sharp $\mathrm{T} / \mathrm{S}$ level crossing resonances. In contrast, the biradical results reviewed below (section e) are characteristic examples of cases with a dynamical distribution of S/T energy gaps.

(d) Homolytic Bond Cleavage and Other Types of Reactions. Photochemical decomposition of dibenzoyl peroxide (15) is one of the early examples of a photochemical MFE in liquid solutions. It was re-

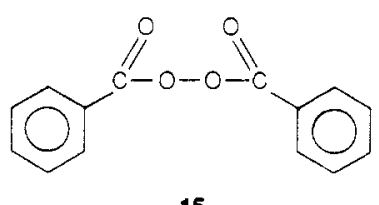

15

ported in 1976 by Hayashi and co-workers ${ }^{130}$ for the singlet-sensitized photolysis of dibenzoyl peroxide in toluene. A large variety of products, mainly escape products, are formed in this reaction. ${ }^{67,322}$ The effects develop clearly only at rather high fields between 1 and $4 \mathrm{~T}$. (A previous attempt ${ }^{337}$ to detect magnetic field effects with this reaction below $1.3 \mathrm{~T}$ had been unsuccessful.) For the cage product phenyl benzoate a case 2 MFD with negative $R$ is observed. It is explained by the $\Delta g$ mechanism, which is corroborated by the square root dependence on the magnetic field, as expected theoretically (cf. section V).

Rather strong effects of the case 1,2 MFD have been found for some escape products, e.g., for 3,3'-dimethylbiphenyl. It has been shown that the dimethylbiphenyls are formed from the solvent toluene via an assisted reaction involving benzoyloxyl radicals. ${ }^{332}$

Norrish type I cleavage of ketones is a common reaction pathway in photochemistry to produce radical pairs. Whereas, however, in micellar solutions such reactions have often been used to study magnetic field effects, in homogeneous solutions there seems to be only one example. Fischer ${ }^{323}$ reported a marked MFE on the yield of pivaldehyde (17), which is a cage product in the

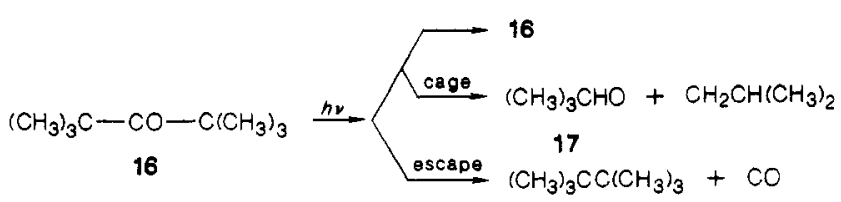

photolysis of di-tert-butyl ketone (16). The special attraction of this reaction as an example of a magnetic-field-dependent reaction lies in the fact that in the tert-butyl radical there are nine magnetically equivalent protons so that an exact expression for the spin motion in general fields may be easily obtained (cf. section V).

The MFE is slightly positive at low fields, which is characteristic of the one-coupling-constant spin system, and is negative at higher fields with a saturation behavior characteristic of the hyperfine coupling mechanism. The theoretical results based on this mechanism reproduce the experimental data quite well, although it is essential to use a correct relaxation rate constant for the pivaloyl radical. This parameter had been determined in previous ESR experiments.

Whereas with one exception related to the triplet mechanism, all MFEs in liquid solution reviewed in this section are manifestations of the radical pair mechanism, recent studies by Ferraudi and co-workers ${ }^{116,772,773}$ have contributed novel mechanistic aspects to photochemically relevant processes in liquid solutions. Applying magnetic field pulses up to $2.4 \mathrm{~T}$, they found marked effects on the quantum yields of the photoaquation of $\left[\mathrm{Rh}\left(\mathrm{NH}_{3}\right)_{5} \mathrm{X}^{-}\right](\mathrm{X}=\mathrm{Cl}, \mathrm{Br})$ and of $\mathrm{K}_{2}[\mathrm{Co}-$ $(\mathrm{CN})_{6}$ ] (cf. Table 8). In the latter case the ratio $k_{\mathrm{R}} / k_{\mathrm{r}}$ of the rate constants of photoreaction $\left(k_{R}\right)$ and radiationless decay $\left(k_{\mathrm{r}}\right)$ increased by a factor of 3 when a magnetic field of $2.4 \mathrm{~T}$ was applied. The explanation 


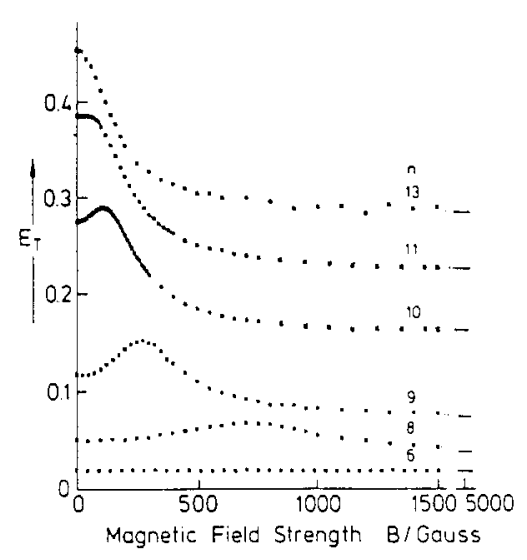

Figure 19. Triplet absorbance observed as a function of magnetic field strength in linked EDA systems pyrene- $\left(\mathrm{CH}_{2}\right)$-DMA (18n) in acetonitrile. Reprinted from ref 309 with kind permission of A. Weller; copyright 1986 Springer-Verlag.

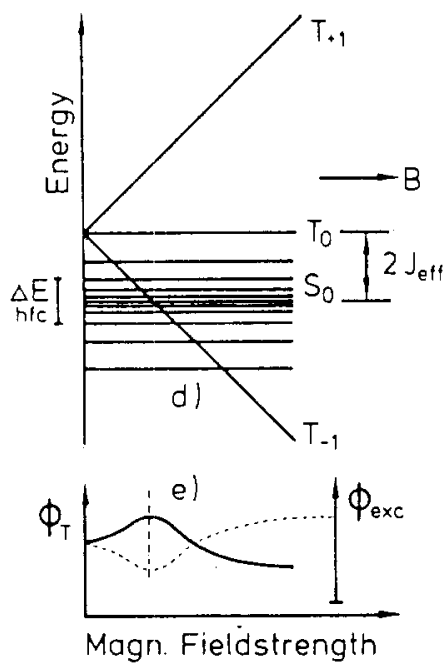

Figure 20. Top (d): General two-electron energy level diagram for the geminate doublet pair. The splitting of the $T_{+1}, T_{0}$, and $T_{-1}$ energy levels of the triplet pair state is due to the Zeeman interaction. Also indicated are singlet-triplet splitting due to exchange interaction $(2 J)$ and the order of magnitude of nuclear hyperfine coupling $\left(\Delta E_{\mathrm{hfc}}\right)$. $J_{\text {eff }}$ is the effective exchange interaction. Bottom: Relative pyrene triplet yield $\Phi_{\mathrm{T}}(-)$ and exciplex yield $\Phi_{e x c}(\cdots)$ as a function of the magnetic field strength obtained for a linked system $\mathrm{A}-\left(\mathrm{CH}_{2}\right)-\mathrm{D}$. Reprinted from ref 309 with kind permission of A. Weller; copyright 1986 Springer-Verlag.

of this effect was sought in terms of the coupling of spin and angular momentum in orbitally degenerate states to the magnetic field.

Authors of this group also studied the influence of intense magnetic fields (pulses of $7 \mathrm{~T}$ ) on the decay kinetics of various phthalocyanine triplets. The kinetics can be described as a superposition of a first-order $T_{1}$ $\rightarrow \mathrm{S}_{0}$ process $\left(k_{1}\right)$ and a second-order $\left(\mathrm{T}_{1}+\mathrm{T}_{1}\right)$ process $\left(k_{2}\right)$ via which radical ion pair formation or excited singlet state formation results. The rate constant $k_{1}$ is increased by a magnetic field, which is explained as the result of a direct magnetic mixing of the $T_{1}\left(E_{u}\right)$ and $\mathrm{S}_{1}\left(\mathrm{E}_{\mathrm{u}}\right)$ state. The rate constant $k_{2}$ is decreased by the magnetic field. This effect is, however, too strong to be explicable in terms of the usual $(T+T)$-pair mechanism in solution, based on the individual triplet spin relaxation processes due to rotational diffusion (Atkins and Evans; ${ }^{338}$ cf. section V). Frink et al. suggested that the $\mathrm{T}-\mathrm{T}$ process should lead to formation of a singlet excimer. There are four nearly degenerate
TABLE 9. Chain Length Dependence of Molecular Triplet Formation in Biradicals (after Weller et al. ${ }^{308}$ )

\begin{tabular}{lcc}
\hline \multicolumn{1}{c}{$\begin{array}{c}\text { chain } \\
\text { length }\end{array}$} & $\begin{array}{c}\text { energy } \\
\text { relations }\end{array}$ & $\begin{array}{c}\text { molecular triplet } \\
\text { formation }\end{array}$ \\
\hline$n \leq 6$ & $2 J_{\text {eff }}>2 J_{\min }>\Delta E_{\mathrm{hf}}$ & none from radical pair \\
$6<n<12$ & $2 J_{\text {eff }}>\Delta E_{\mathrm{hn}}>2 J_{\min }$ & $\Phi_{\mathrm{T}}\left(B_{\mathrm{M}}\right)>\Phi_{\mathrm{T}}(B=0)>$ \\
& $\Phi_{\mathrm{T}}(B \rightarrow \infty)$ \\
$n \geq 12$ & $\Delta E_{\mathrm{hfi}}>2 J_{\text {eff }}>2 J_{\min }$ & as in unlinked systems
\end{tabular}

orbital states in $a^{1}\left({ }^{3} E_{u}+{ }^{3} E_{u}\right)$ encounter pair, which are assumed to exhibit largely different activation energies for excimer formation. The MFE is assumed to come in through a magnetic splitting of the four ${ }^{1}\left({ }^{3} \mathrm{E}_{\mathrm{u}}+{ }^{3} \mathrm{E}_{\mathrm{u}}\right)$ states. These would correspond to the $\{i\}$ manifold in terms of the general mechanistic view developed in section II.

(e) Biradicals. In some of the systems listed in Table 8, the radical pairs originating from the respective photochemical primary reactions are linked by a polymethylene chain. There has been considerable interest in the dynamical and spin properties of such biradical species, ${ }^{42,339,340}$ and the study of MFEs on the decay of such species is expected to be particularly instructive as far as spin exchange interaction, intersystem crossing mechanisms, and the dynamics of the chain end-to-end distances are concerned.

Weller and co-workers ${ }^{98,308,309,766}$ investigated the pyrene/dimethylaniline pair linked by a polymethylene chain of $n=6-12(18 n)$. As for the separate donor/

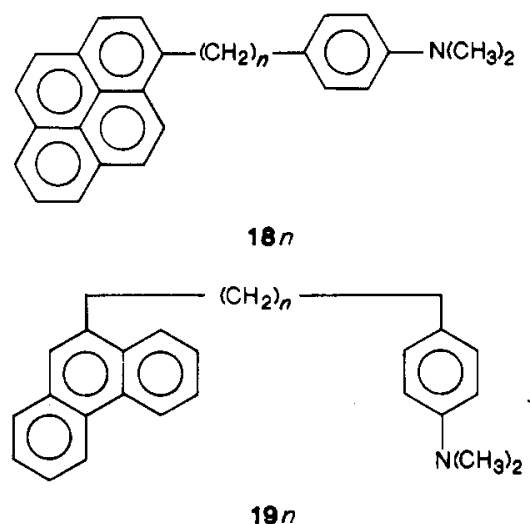

acceptor system, singlet radical pairs are formed initially, which after crossing to the radical pair triplet states may recombine to yield a certain amount of pyrene triplets. The MFD found for the triplet yield is of case 1,3 type (cf. Figure 19). It is typical of the level crossing between a triplet Zeeman component and the radical pair singlet, which, in zero field, are split by the exchange energy $2 J$. Depending on the length of the polymethylene chain, three cases may be distinguished (cf. Table 9).

To evaluate the effects it was assumed that $B_{\mathfrak{m}}$, the maximum position of the MFD, indicates an effective value $2 J_{\text {eff }}$ characteristic of the most frequently occurring end-to-end distance $r_{\text {eff }}$. The latter was calculated from an exponential distance law:

$$
2 J(r)=2 J_{0} \exp (-\alpha r)
$$

with the result that the $r_{\text {eff }}$ values are proportional to the square root of the number of methylene links. This result appears quite reasonable. However, as was pointed out by Bittl et al., ${ }^{347}$ the field $B_{\mathrm{m}}$ of the MFD maximum for these biradicals cannot be adequately 


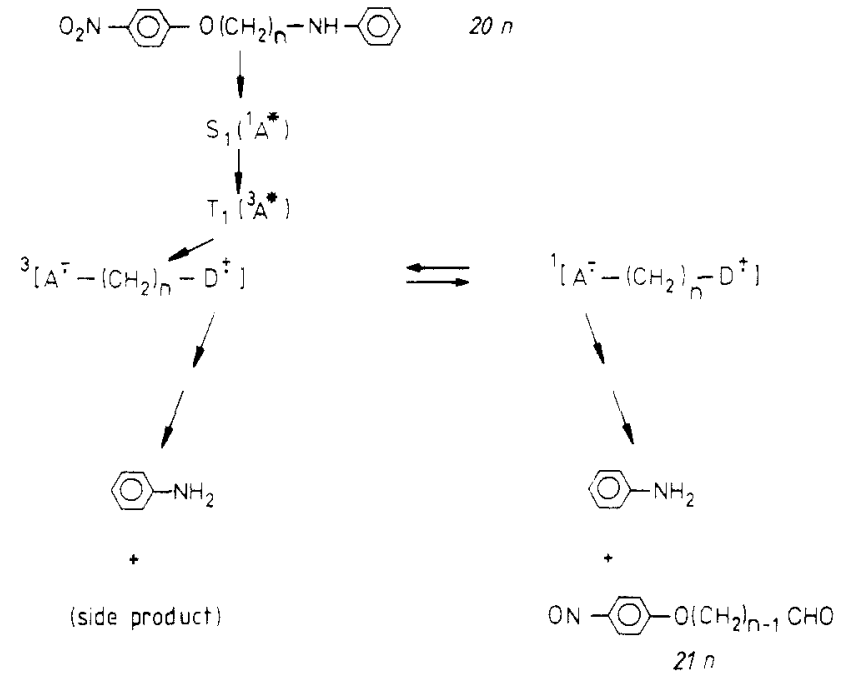

Figure 21. Reaction scheme for magnetic-field-dependent photolysis of linked electron donor-acceptor systems 20n (after Nakagaki et al. ${ }^{317}$ ).

interpreted without accounting for the dynamics of the end-to-end distance (cf, also below and section V).

Another singlet biradical system originating from photoelectron transfer in the linked compound $19 n$ was investigated by Tanimoto et al., ${ }^{351}$ observing the emitted exciplex fluorescence intensity. For comments on this system, cf. section 3 .

A biradical system generated in the triplet spin state by intramolecular electron transfer was investigated by Nakagaki et al. ${ }^{317}$ (cf. Figure 21). The reaction was followed by stationary photokinetics. The quantum yield of disapeparance of the starting material decreases in a magnetic field, which indicates an initial triplet multiplicity of the radical pairs. MFEs are observed only for $n \geq 8$. Again, this points to the distance dependence of the exchange interaction, which is prohibitive for magnetic-field-sensitive intersystem crossing processes at short end-to-end distances.

Examples of photochemical MFEs due to triplet biradical intermediates that are produced by intramolecular H-atom transfer have been reported by Tanimoto et al. ${ }^{327,352}$

The anthraquinone derivative 22 undergoes photoreductive bleaching, ${ }^{327}$ whereby, among other products involving reaction with molecular oxygen present in the solution, a cyclic ether $\mathbf{2 3}$ is formed as an intramolecular coupling product from the biradical. The $\mathrm{H}$ abstraction

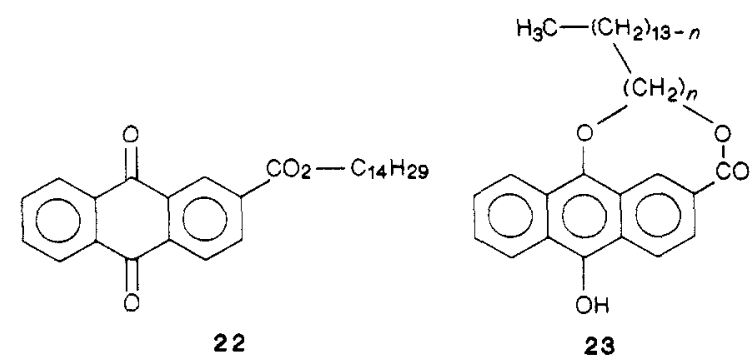

is probably not very selective for the $H$ position in the terminal part of the $\mathrm{C}_{14}$ chain. However, occurrence of a MFE $R \leq-12 \%$ with $B_{1 / 2} \approx 15 \mathrm{mT}$ for the bleaching yield indicates that there should be major contributions from $\mathrm{C}-\mathrm{H}$ positions that may separate far enough from the semiquinone radical spin in the extended-chain conformation.
Another type of H-abstraction-derived biradical 25n, recently reported by Tanimoto et al., ${ }^{352}$ is produced by photoexciting linked systems of type $24 n$. Here the

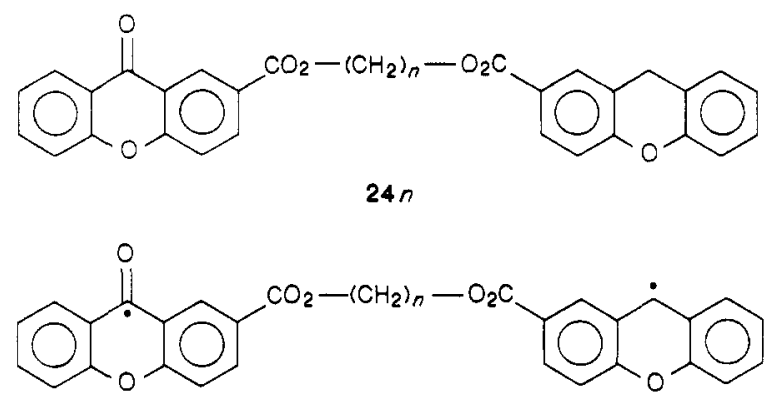

$25 n$

radical positions are well defined. For $n=2-12$ the biradical decay has been directly observed by laser flash spectroscopy. In zero field the decay times $(0.1-0.22$ $\mu \mathrm{s}$ for $n>2,0.98 \mu \mathrm{s}$ for $n=2$ ) are longer than expected for the ISC process on the basis of the hfc mechanism. However, very large MFEs on the decay times ensue, even for $n=2$ (cf. Table 8). These effects increase with $n$, leveling off at $n=12$, where the lifetime is increased by a factor of 20 in a field of $0.8 \mathrm{~T}$ as compared to zero field. The MFD of $\tau$ increases monotonously and is not saturated at $0.8 \mathrm{~T}$. Spin-orbit coupling, as invoked to explain the decay rates of some acyl-alkyl biradicals (vide infra), does not seem to be of importance here. The effects are attributed to a combination of the hfc and the relaxation mechanism.

Biradicals with varying end-to-end distances may be conveniently produced by using Norrish type I cleavage reactions of alicyclic ketones. Magnetic interaction in biradicals from such reactions was first studied by Closs and Doubleday ${ }^{341}$ in 1973 , using the CIDNP method. A number of further investigations of this type have been reported since. ${ }^{342-345}$ It was found that the CIDNP intensity passes through a maximum at a field strength $B_{\mathrm{m}}$, increasing with decreasing chain length.

While this behavior reflects the general distance dependence of exchange interaction between biradical termini, it was learned from a detailed theoretical analysis of the MFD of CIDNP intensity by de Kanter et $\mathrm{al}^{346}$ (cf. also section V) that the positions and widths of the CIDNP maxima do not simply reflect the quasi-stationary distribution of exchange interaction, as would be expected from the end-to-end distance distribution, but depend critically also on the chain dynamics. This point has also been confirmed in a recent theoretical study by Schulten and co-workers. ${ }^{347}$

It has also been concluded from CIDNP experiments that triplet-singlet transitions in biradicals of medium and short chain length are largely due to the spin-orbit coupling mechanism, which is not sensitive to a magnetic field, and most efficient in diradical conformations with close end-to-end approach. Therefore CIDNP intensities strongly decrease with decreasing chain length.

Recently, Closs et al. ${ }^{348}$ applied time-resolved CIDNP to probe biradical decay. The grow-in times of nuclear polarization for cyclic coupling and acyclic disproportionation products from 1.6 and 1.8 biradicals were found to be of the order of $100 \mathrm{~ns}$.

The first direct time-resolved detection of alkyl-acyl biradicals was reported by Turro and co-workers ${ }^{324,349,350}$ 


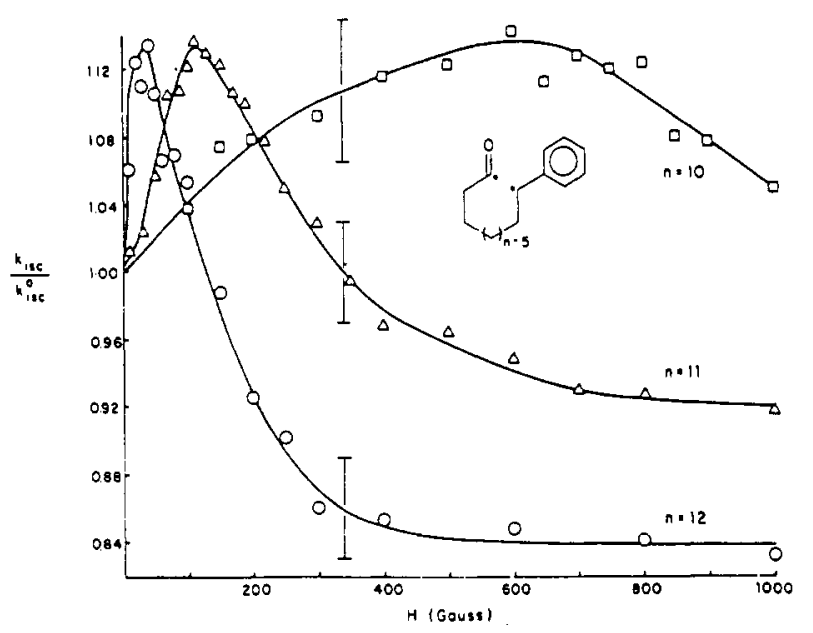

Figure 22. MFD of the ISC rate constant $K_{\text {ISC }}$ for biradicals 27n $(n=10-12)$ relative to the rate constant in the earth's field, $k_{\mathrm{ISC}}{ }^{0}$. Reprinted from ref 349 with kind permission of N. J. Turro, Columbia University, New York; copyright 1985 American Chemical Society.

using laser flash photolysis to investigate the reactions of cycloalkanones (26n). The biradical decay constants

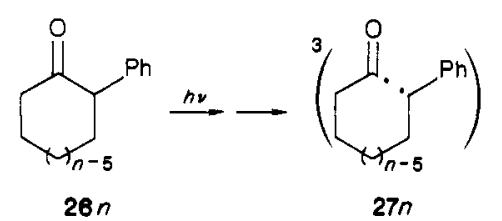

were found around $10^{7} \mathrm{~s}^{-1}$, in agreement with the CIDNP results of Closs et al. ${ }^{348}$ Furthermore, the MFD of biradical decay could be observed directly for chain lengths of $n=10-12$ (cf. Figure 22). It has been stated that the observed magnetic-field-dependent decay constants at room temperature are in fact determined by the triplet-singlet transition process. Conformational changes take over the role of the rate-determining step only at lower temperatures. ${ }^{350}$

The MFD of $k_{\text {ISC }}$ as shown in Figure 22 passes through a maximum of $R=+13 \%$ independent of $n$; however, the position $B_{\mathrm{m}}$ of the maximum sensitively increases with decreasing $n$. At high fields the magnetic field effect saturates at $R=-9 \%(n=11)$ or $-16 \%(n$ $=12$ ). The falling off of the decay rate to values smaller than at zero field reflects the decoupling of $T_{ \pm}$from the $\mathrm{T} \rightarrow \mathrm{S}$ transition. One should expect, however, that in the high-field limit $k_{\mathrm{ISC}}$ should drop to $1 / 3$ of its zerofield value (actually the decay should become biexponential, displaying the markedly distinct decay kinetics of $T_{0}$ as opposed to $T_{ \pm}$). According to the arguments of Turro and co-workers, ${ }^{324}$ the value of $k_{\mathrm{ISC}}$ contains a magnetic-field-independent spin-orbit coupling contribution to $k_{\mathrm{ISC}}$, which has been evaluated on the assumption that in high magnetic fields the hfc contribution drops to $1 / 3$ of its value at zero field. Thus ratios of $76 / 24(n=12)$ and $86 / 14(n=11)$ of soc/hfc contributions to $k_{\mathrm{ISC}}$ have been obtained at zero field.

Furthermore, it has been pointed out that hfc-induced ISC will predominate in the extended-chain conformation, where $2 J$ is small, whereas the soc-induced ISC occurs preferentially at close end-to-end conformations. This should be kept in mind when attempting a quantitative analysis of the $B_{\mathrm{m}}$ values.

\section{Photoluminescence and Chemiluminescence}

Luminescence in liquid solutions, although not necessarily indicative of irreversible chemical change, may be conveniently used to probe the MFD of processes that are also of general importance in photochemical reaction mechanisms. Magnetic field effects on luminescence in liquid solutions are usually due to pair mechanisms involving two doublets (radical pair), two triplets, or a mixed triplet-doublet pair. Intramolecular mechanisms such as the triplet mechanism, which is a common one in low-temperature solid-state luminescence, have so far not been found effective in liquid solution luminescence.

(a) Radical Pairs. The recombination of two radicals, preferably radical ions, may produce electronically excited species, which can give rise to luminescence. In some cases the recombining radical ion pairs, produced, e.g., by electrochemical methods or by radiolysis (cf. section IV.C.4), have sufficient energy to directly populate an excited singlet state. In most cases, however, the energy is just sufficient to populate the lowest excited triplet of one of the recombination products. Luminescence may then arise from triplet-triplet annihilation. MFEs observed under such conditions are usually characteristic of pair processes involving triplet-triplet or triplet-doublet interaction and have been used to discriminate between the singlet and triplet routes of electrogenerated chemiluminescence $(E C L)^{353-355}$ (vide infra).

Whereas in random radical ion recombinations magnetic effects according to the radical pair mechanism are small, they are more pronounced if the recombining radicals are of geminate origin. The work of Weller and co-workers ${ }^{302-306}$ on the triplet recombination products of singlet radical pairs produced by quenching of aromatic singlets by electron donors or acceptors provides a number of examples. Here the triplet yield has been monitored via delayed fluorescence arising from triplet-triplet annihilation. It has been shown that the magnetic field modulation of this luminescence intensity is due to the magnetic field effect on the recombination of the geminate radical ion pairs (cf. last section), whereby the excited triplets are generated. An increase in triplet formation efficiency will cause higher stationary triplet concentrations. The observed intensity of the delayed fluorescence is proportional to the square of the triplet concentration. The MFE on the radical pair recombination saturates at fields typically below $0.1 \mathrm{~T}$, where magnetic field effects due to the $\mathrm{T}+\mathrm{T}$ and the $\mathrm{T}+\mathrm{D}$ mechanisms are generally still negligible.

In the pyrene anion/aromatic amine cation radical ion pairs the fluorescing singlet exciplex state is energetically accessible. In fact, a quasi-equilibrium between singlet geminate radical ion pair and exciplex should be rapidly established so that the population of singlet radical pairs may be probed by the exciplex fluorescence intensity. A magnetic field that impedes singlet-triplet transitions in the radical pairs should increase the stationary concentration of the singlet pairs so that this effect becomes detectable through the exciplex fluorescence intensity.

MFEs on exciplex fluorescence intensity have been indeed observed by Frankevich and co-workers ${ }^{356,357}$ and by Nath and Chowdhury. ${ }^{358}$ The effects are, however, 


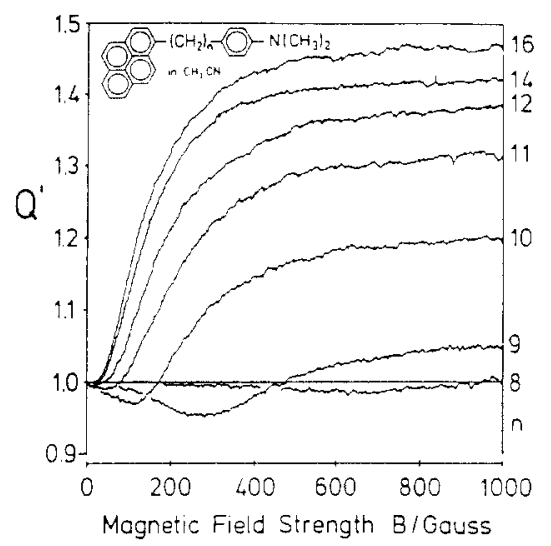

Figure 23. MFD of the relative exciplex fluorescence intensity $Q^{\prime}=I^{\prime}(B) / I^{\prime}(0)$ of compounds $18 n(n=8-16)$ in acetonitrile. Reprinted from ref 98 with kind permission of A. Weller; copyright 1985 Elsevier Science Publishers B.V.

very small (about $1 \%$ ) because the lifetime of the geminate pairs is determined by fast diffusive separation rather than by the singlet/triplet transition process. The effect depends on solvent polarity. Petrov et al..$^{357}$ studied this magnetic field effect in various alcohols and alcohol mixtures. It passes through a maximum for a dielectric constant of $26(R(8 \mathrm{mT})=+3 \%)$. Their results allowed them to evaluate an activation energy of about $0.2 \mathrm{eV}$ for exciplex formation from the singlet radical pair.

Much stronger effects on exciplex fluorescence ensue if donor and acceptor radicals are linked together by a polymethylene chain. For such systems enhancements of exciplex fluorescence intensity up to $50 \%$ in a magnetic field of less than $0.1 \mathrm{~T}$ have been reported by Staerk et al.$^{98}$ and Tanimoto et al. ${ }^{351}$ In Figure 23 is shown the MFD of the exciplex fluorescence intensity in the covalently linked electron donor/acceptor system $18 \mathrm{n}$ studied in Weller's group. ${ }^{98}$ Maximum increases of the exciplex fluorescence are observed with the longest linkage of 16 methylene groups. As the chain gets shorter the maximum of the effect decreases and the half-field value increases. Even sign inversion at low field strength occurs. These effects are quite parallel to the corresponding effects on the triplet yield from the radical pair geminate recombination (cf. last section) and are due to the interplay of diffusion and exchange interaction.

In the linked electron donor-electron acceptor systems $19 n$ investigated by Tanimoto et al ${ }^{351}$ the series with $n=3,6,8$, and 10 was investigated. The CT-type emission band of these compounds consisted of two slightly shifted spectral components with different lifetimes, which could be also distinguished by differences in their excitation spectra. They were attributed to excitation of an EDA complex conformer, already present in the ground state, and to the exciplex dynamically generated after excitation of an extended conformation of the linked system. Only the latter component, showing the longest fluorescence lifetime, exhibited a MFE, which for $n=10$ and $n=8$ was similar to the cases $n \geq 14$ and $n=9$ in the pyrenyl series, respectively. For $n=3$ and $n=6$ no magnetic field effect was found, which is in line with CIDNP and MFE studies in other biradical systems.

MFEs in chemiluminescent reactions with luminol $\left(\mathrm{LH}_{2}(\mathbf{2 8})\right)$ have been studied by Russian workers. ${ }^{113,376}$
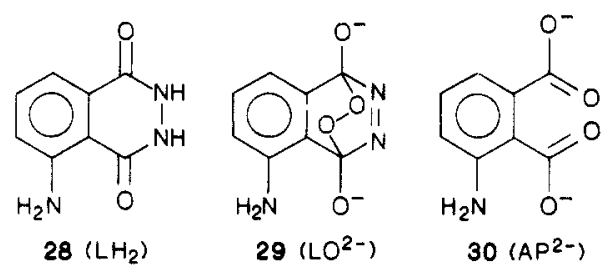

The chemiluminescence induced by oxidation of luminol with potassium ferricyanide showed a very weak $\operatorname{MFE}\left(R_{\mathrm{S}} \approx+0.16 \%, B_{1 / 2} \approx 3 \mathrm{mT}, B_{\mathrm{S}} \approx 6 \mathrm{mT}\right)$, that required very sophisticated experimental equipment (cf. section III) in order to be reliably detected. ${ }^{113}$ The chemiluminescence is thought to be due to the following reaction steps:

$$
\begin{gathered}
\mathrm{LH}_{2}+2 \mathrm{OH}^{-} \rightarrow \mathrm{L}^{2-}+2 \mathrm{H}_{2} \mathrm{O} \\
\mathrm{L}^{2-}+\mathrm{Fe}(\mathrm{CN})_{6}^{3-} \rightarrow \mathrm{L}^{--}+\mathrm{Fe}(\mathrm{CN})_{6}^{4-} \\
2 \mathrm{~L}{ }^{--}+\mathrm{O}_{2} \rightarrow \mathrm{LO}_{2}{ }^{2-}+\mathrm{L} \\
\mathrm{LO}_{2}{ }^{2-} \rightarrow\left[\mathrm{AP}^{2-}\right]^{*}+\mathrm{N}_{2}
\end{gathered}
$$

The MFE is tentatively explained by the radical pair mechanism suggested to operate in the homogeneous disproportionation reaction of $\mathrm{L}^{-}$radicals. Since a magnetic field effect reduces the recombination efficiency of a random radical pair, the stationary concentration of the luminol radicals will increase and so will the luminescence intensity.

Similarly, small MFEs $(+0.25 \%$ to $+0.5 \%)$ were found in the chemiluminescence from luminol and 4(dimethylamino)phthalhydrazide when their oxidation was photosensitized with methylene blue triplets. ${ }^{376}$ Here the effects are explained in terms of efficiency of dissociation of geminate triplet radical pairs from methylene blue semiquinone and substrate radical.

(b) Triplet-Triplet Pairs. MFEs on the efficiency of triplet-triplet annihilation in liquid solution were first reported by Faulkner and Bard. ${ }^{359}$ The delayed fluorescence from photoexcited anthracene showed a monotonic decrease with magnetic field strength $\left(R_{\mathrm{DF}}(0.8 \mathrm{~T}) \approx-5 \%\right)$. This feature was also borne out in experiments of Avakian et al., ${ }^{360}$ who sensitized anthracene fluorescence by triplet-triplet energy transfer from eosin triplets. The positive MFE at low fields as observed in molecular crystals was missing in liquid solutions. This has been attributed to the random orientations at which triplet pairs encounter in liquid solution. ${ }^{360}$ For more theoretical details of the interpretation, cf. section V.

For pyrene and some other aromatic compounds monomer and excimer bands may be simultaneously detected in the delayed fluorescence. Here it is an interesting problem whether the excimers are directly generated in the triplet-triplet annihilation process or subsequently in $S_{1}+S_{0}$ encounters. In the former case some difference in the MFD of delayed fluorescence can be expected, since direct excimer formation in triplettriplet annihilation might require more specific pair geometries than the $\mathrm{T}+\mathrm{T} \rightarrow \mathrm{S}_{1}+\mathrm{S}_{0}$ energy-transfer process.

Investigating the delayed fluorescence of pyrene and 1,2-benzanthracene at room temperature, Tachikawa and Bard ${ }^{361}$ found identical MFDs for monomer and excimer emission. However, Wyrsch and Labhart ${ }^{362}$ 
reported that the MFD of the monomer and excimer delayed fluorescence was different for 1,2-benzanthracene at temperatures between -70 and $-170{ }^{\circ} \mathrm{C}$. In this case the delayed fluorescence increased by $4 \%$ in a magnetic field of $40 \mathrm{mT}$. At higher fields the effect remained constant for the excimer and decreased for the monomer. A few years later the same group published a very detailed investigation on the delayed fluorescence of pyrene, 1,2-benzanthracene, 3,4-benzopyrene, and phenanthrene in various solvents between -140 and $0^{\circ} \mathrm{C}$ covering a range of viscosities from 2 to $50 \mathrm{cP}$. ${ }^{69}$ The experiments afforded the separate MFD of the rate constants $k_{\mathrm{e}}, k_{\mathrm{m}}$, and $k_{2}=k_{\mathrm{r}}+k_{\mathrm{e}}+k_{\mathrm{m}}$ defined in scheme 56. At higher temperatures the

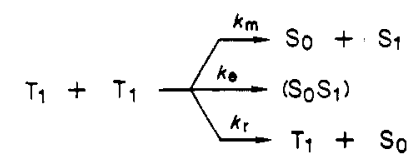

MFEs on monomer and excimer formation are identical within the limits of experimental error for any solvent or excimer-forming solute investigated. At lower temperatures the effects on monomer and excimer fluorescence start to diverge, which the authors attribute to specific restrictions in triplet-triplet pair conformations leading preferentially to excimer formation.

Furthermore, an initially positive magnetic field effect at low fields has been shown to be quite general at high solvent viscosities. Such a MFD has also been reported by van Willigen ${ }^{364}$ for pyrene. According to a detailed theoretical analysis by Lendi et al., ${ }^{69}$ it should be explained as a consequence of the triplet pairs' singletquintet energy splitting. It is a level-crossing effect, which is, however, borne out only under conditions where the triplet pair lifetime is fairly long (for details, cf. section V).

Another case of triplet-triplet interaction, particularly important in photochemistry, is encountered in the quenching of excited triplets by molecular oxygen. Experiments by Tachikawa and Bard ${ }^{365}$ on the delayed fluorescence of anthracene and pyrene exhibited a sign inversion of the MFE from negative at low oxygen concentrations to positive at higher oxygen concentrations. This could be an indication of the impeding effect of a magnetic field on the reaction

$$
{ }^{3} \mathrm{~T}+{ }^{3} \mathrm{O}_{2} \rightleftarrows{ }^{1,3,5}\left(\mathrm{~T} \mathrm{O}_{2}\right) \rightarrow \mathrm{S}_{0}+{ }^{1} \mathrm{O}_{2}
$$

which would account for the observation, if quenching by oxygen determines the triplet lifetime. The effect is only seen in DMF but not in acetonitrile.

The problem of how the rate of triplet quenching by oxygen should depend on a magnetic field has been theoretically investigated by Stone and Swenberg ${ }^{366}$ and by Geacintov and Swenberg, ${ }^{367}$ who pointed out that CT interactions in the $\left(\mathrm{T}^{3} \mathrm{O}_{2}\right)$ pair might cause rahter large singlet-quintet level splittings, which might prohibit any transition between them so that no magnetic field effects are to be expected. In fact, Swenberg and Geacintov ${ }^{367}$ failed to see magnetic field effects on oxygen quenching when investigating the delayed fluorescence of polynuclear aromatics adsorbed on polystyrene.

(c) Triplet-Doublet Pairs. The rate constants of $(T+D)$-pair reactions (usually triplet quenching by radicals) leading to a $(S+D)$ pair of products (eq 58 ) depend on the distribution of doublet character over

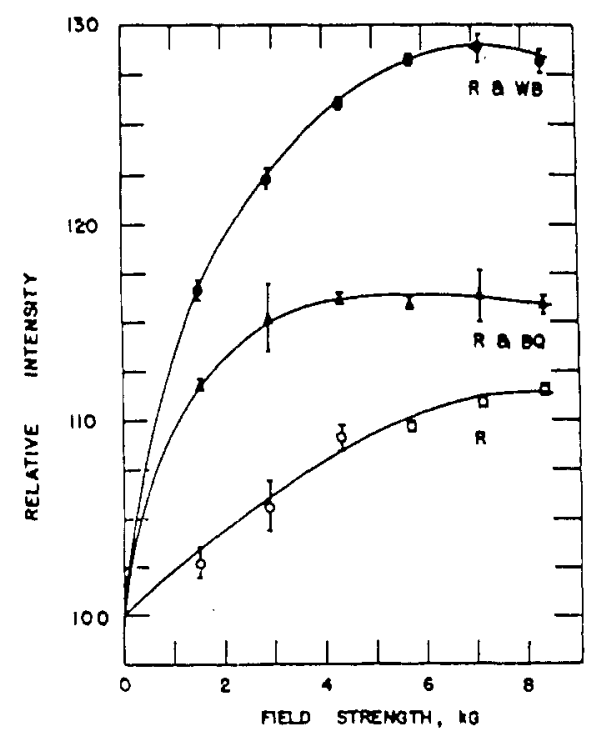

Figure 24. MFEs on electrogenerated luminescence from systems containing rubrene as an emitter. Reactants: (e) rubrene anion and Wurster's blue cation; ( $\Delta$ ) rubrene cation and $p$-benzoquinone anion; $(0)$ rubrene anion and cation radicals. Reprinted with permission from ref 354; copyright 1972 American Chemical Society.

the pair states during $(T+D)$ encounters. In the

$$
\mathrm{T}_{1}+\mathrm{D} \rightleftharpoons{ }^{2,4}\left(\mathrm{~T}_{1} \mathrm{D}\right) \rightarrow \mathrm{S}_{0}+\mathrm{D} \text { (or } \mathrm{D}^{*} \text { ) }
$$

presence of a magnetic field pair states with total doublet spin are concentrated on fewer pair eigenstates than in zero field so that, according to the Merrifield model, a magnetic field reduces the average rate constant of such processes.

Effects of this kind have been reported by Faulkner and Bard $^{368}$ for the delayed fluorescence of anthracene in the presence of Wurster's blue cations (TMPDA ${ }^{*+}$ ). At a radical concentration where the quenching process (58) determines the lifetime of the triplets, a monotonic increase of delayed fluorescence is observed, which is explained by the impeding effect of the magnetic field on the triplet + doublet quenching process, leading to a higher stationary triplet concentration and hence to a higher delayed fluorescence intensity $(R(0.8 \mathrm{~T}) \approx$ $+2 \%)$. The change from a $(\mathrm{T}+\mathrm{T})$-type MFD of delayed fluorescence intensity to a $(T+D)$-type behavior has been demonstrated with variable concentration of radical quenchers (TMPDA ${ }^{*+}$, benzoquinone anion radical) by Tachikawa and Bard. ${ }^{369}$

The simultaneous presence of excited triplets and high concentrations of radical ions is a typical situation in electrogenerated luminescence, where radical ions produced in electrode processes recombine to produce excited states. A classification of ECL systems may be based on the criterion whether the ion recombination energy is sufficient to produce excited singlet states (S route) or only triplet states ( $\mathrm{T}$ route). As has been shown by Bard and co-workers, ${ }^{353-355}$ MFEs may be used to distinguish between these routes (for reviews of this work, cf. Atkins and Lambert, ${ }^{17}$ Faulkner, ${ }^{21}$ Sagdeev et al.,22 and Salikhov et al. ${ }^{52}$ ). Only energydeficient systems, i.e., those following the $T$ route, showed MFD of ECL. In these cases positive MFEs up to $+30 \%$ (rubrene ECL from rubrene anion radical and TMPDA $\left.^{\circ+}\right)^{354}$ have been observed (cf. Figure 24), which are attributed to the $(T+D)$ mechanism. For S-route systems MFEs according to the radical pair mechanism 
might be expected. Such effects have, however, not been reported under ECL conditions.

In ECL systems that are not too strongly energy deficient (e.g., rubrene cation + anion ${ }^{361}$ changes of solvent ${ }^{261}$ or supporting electrolyte concentration ${ }^{370}$ may cause a shift from the $T$ route to the $S$ route, which is borne out in a decrease of the MFE.

Magnetic field effects in ECL have also been reported by Santanham and co-workers. ${ }^{371-373}$ In ECL systems involving riboflavin anion with rubrene cation, the recombination fluorescence from rubrene exhibited a marked MFE $\left(R_{\mathrm{DF}}(0.4 \mathrm{~T})=+28.6 \%\right)$, taken as evidence that the luminescence is generated via the $T$ route. ${ }^{373}$

There are also exceptions to the "energy deficiency rule" for magnetic field effects in ECL. Periasamy et al. $^{375}$ observed a large MFE $(R(1 \mathrm{~T})=+300 \%)$ in the ECL generated via phenanthrene cation/anion radical recombination, although in this system the ion recombination energy exceeds the phenanthrene $S_{1}$ energy by $0.6 \mathrm{eV}$. As an explanation it was pointed out that even if the recombination energy is sufficient to populate the $\mathrm{S}_{1}$ state, formation of triplets is not excluded. Furthermore, phenanthrene shows very efficient intramolecular triplet formation $\left(\Phi_{I S C}=0.8\right)$, allowing for a considerable contribution of emission in a subsequent triplet-triplet annihilation process.

A very particular situation has been investigated by Razi Naqvi et al., ${ }^{374}$ who studied triplet-doublet energy transfer from triplet benzophenone to benzophenone ketyl radicals. Since fluorescence intensity from the benzophenone ketyl radicals could be observed, this provided a direct measure of the efficiency of the $\mathrm{T}+$ $\mathrm{D}$ process. The emission intensity is increased by $1 \%$ in a field of about $100 \mathrm{mT}$ and is decreased to values lower than at zero field at about $300 \mathrm{mT}$. Whereas the decreasing part is in accord with what is expected from simple theories of triplet-doublet interaction, no definite mechanistic interpretation was offered for the initial rise of the MFD.

\section{Radioluminescence}

When high-energy radiation (X-and $\gamma$-rays, fast $\alpha$ and $\beta$-particles) is absorbed in condensed matter, a number of ionization events occur along the track of the incident particles whereby cation and anion radicals of suitable solutes may be finally formed, which on recombination give rise to scintillation pulses or steadystate radioluminescence ${ }^{33,46,56,389,390}$ At high solute concentrations the main reactions following the primary reaction (59) $\operatorname{are}^{391}(\mathrm{~S}=$ solvent, $\mathrm{M}=$ solute)

$$
\begin{gathered}
\mathrm{S} \rightarrow \mathrm{S}^{+}+\mathrm{e}^{-} \\
\mathrm{S}^{+}+\mathrm{M} \rightarrow \mathrm{S}+\mathrm{M}^{+} \\
\mathrm{e}^{-}+\mathrm{M} \rightarrow \mathrm{M}^{-} \\
\mathrm{M}^{+}+\mathrm{M}^{-} \rightarrow \mathrm{M}^{*}+\mathrm{M}
\end{gathered}
$$

If the energy deposited in reaction 59 is high, the secondary electron may cause further ionizations so that the spur comprises more than one ion pair. In general, the spur size depends on the energy and charge of the primary particles and the type of solvent. ${ }^{390}$ In alkanes the secondary electron from reaction 59 will travel a typical distance of about $7 \mathrm{~nm}$ before it is scavenged.
Yet the radical pair thus produced is within the Onsager escape distance of about $30 \mathrm{~nm}$, and charge recombination will be mostly geminate. Since in nonpolar solvents the recombination energy of ion pairs is very high, reaction 62 is diffusion controlled. Furthermore, the multiplicity of the excited state $M^{*}$ is not subject to energy restrictions but depends only on the spin correlation of the radical pair prior to recombination. The total electron spin of a spur is singlet. ${ }^{389}$ If, however, more than one pair is in a spur, various combinations of individual radical pairs from it may have also initial triplet spin correlation. The problem of calculating the $\mathrm{S} / \mathrm{T}$ ratio of possible pairs within such spurs has been a matter of some debate, ${ }^{392-395}$ which does not seem to be completely settled. Nevertheless, it is clear that, as the spur size increases, the $\mathrm{T} / \mathrm{S}$ ratio will approach the statistical limit of 3 . For actual situations, however, the T/S ratio is smaller than 3 and the recombining pair will show spin correlation effects typical of initial singlet radical pairs. ${ }^{396}$

The recombination rate of singlet radical pairs is directly reflected in the intensity of radioluminescence emitted. The suggestion that an effect of initial spin correlation and successive spin evolution, which might be modified by external magnetic fields, should be detectable in the radioluminescence was first advanced by Brocklehurst. ${ }^{397}$ It is of interest to note that this suggestion was made at the same time, but independently of the suggestions of the radical pair mechanism invoked to explain the CIDNP effects. Whereas Brocklehurst first thought that spin evolution and its magnetic field dependence should be described in terms of spin relaxation, some years later he established the relation with the CIDNP-type spin motion, showing that a coherent process driven by isotropic hyperfine coupling was essentially responsible for the observed effects. ${ }^{391}$

Spin correlation and magnetic field effects have been dealt with in several review papers. ${ }^{33,46,52,56,761}$ Nevertheless, we think it useful to document the state of investigations in this field by a comprehensive list of systems studied (cf. Table 10) together with some brief comments on various aspects of the work. We have not included here investigations applying magnetic resonance methods (RYDMR) since these will be covered in section IV.G.

To investigate MFEs radioluminescence has been observed by stationary methods (when excited by $\gamma$ radiation or a $\beta$-source) or by time-resolved fluorescence measurements when excited with $e^{-}$pulses from electron accelerators. Time profiles of scintillations have been measured by the single-photon-counting technique.

The first MFE in radioluminescence was reported with pulse radiolysis for fluorene as emitting species in squalane, a highly viscous aliphatic solvent. ${ }^{37,378}$ In time-resolved measurements it was shown that the fluorescence intensity increased by about $40 \%$ in a magnetic field of $0.3 \mathrm{~T}$ and the triplet recombination yield, detected by transient absorption, increased by $10 \%$.

Fluorene was also investigated in cyclohexane and in benzene, observing steady-state fluorescence under $\gamma$-irradiation. ${ }^{379}$ The MFE is largest with squalane and zero with benzene. In the latter solvent no radical ions of the solute are produced. The energy is directly 
TABLE 10. Magnetic Field Effects on Radioluminescence in Solution

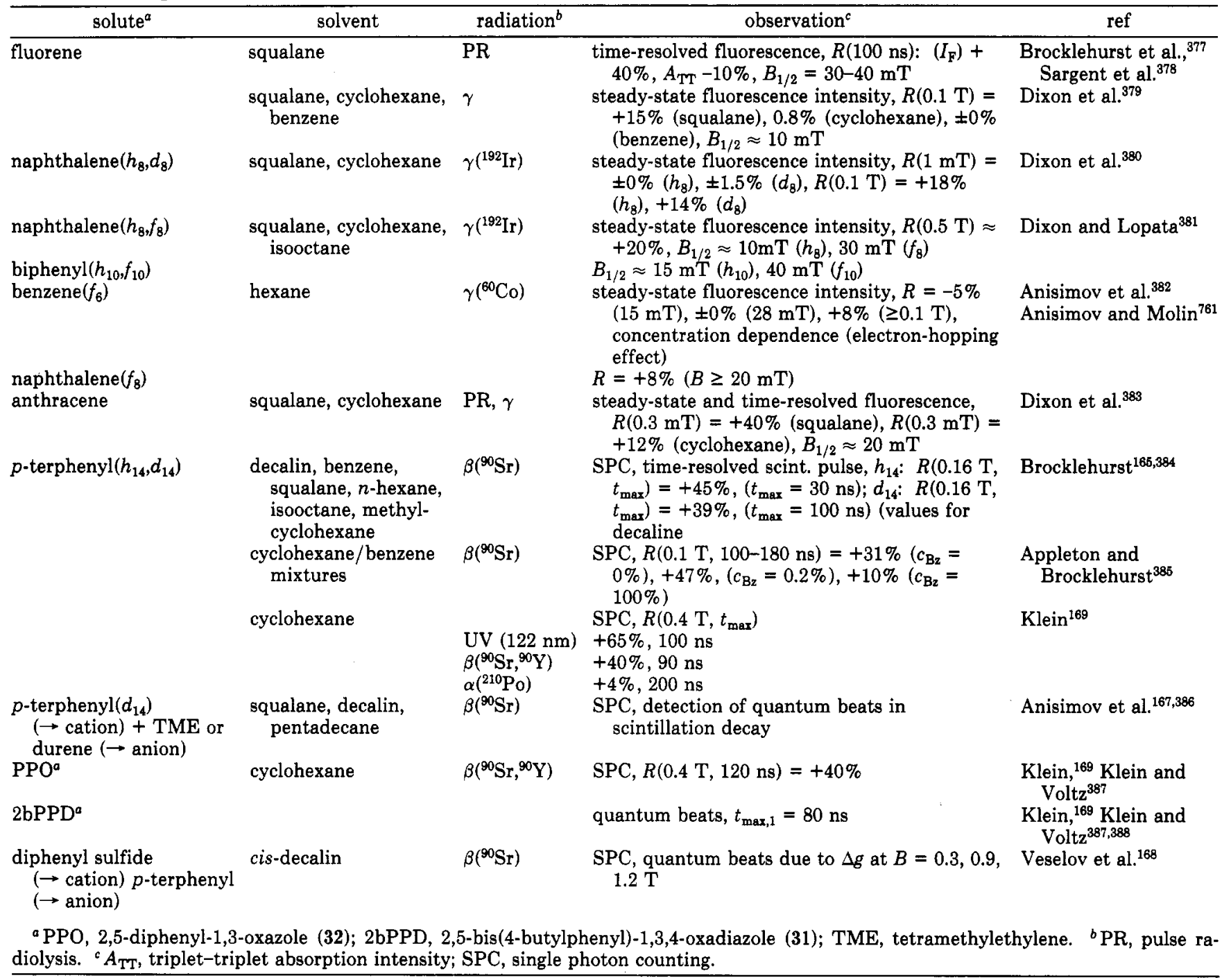

transferred to fluorene from excited states of the solvent.

Other steady-state fluorescence investigations were performed with naphthalene and its deuteriated or perfluorinated derivatives ${ }^{380-382}$ and also with biphenyl and its perfluorinated derivative. ${ }^{381}$ These investigations have shown that the larger the hyperfine coupling ( $\mathrm{F}>\mathrm{H}>\mathrm{D}$ ), the larger is the $B_{1 / 2}$ value.

Radioluminescence of perfluorinated benzene in hexane, which has been investigated by Molin's group,,$^{382}$ is of special interest in that only nuclei with equal and rather strong hfc constant govern the spin evolution. For this case theory predicts a MFD that passes through a minimum at low fields before it changes spin and saturates with a positive MFE at high fields. ${ }^{52}$ These features are clearly borne out by the experimental results.

Time-resolved measurements with the single-photon-counting technique have revealed that the time necessary to develop a magnetic field effect on radioluminescence is typically some $10-100 \mathrm{~ns}$, whereby the growing-in time of the MFE depends on the strength of the hyperfine coupling as first demonstrated by Brocklehurst ${ }^{165,384}$ for $p$-terphenyl and its perdeuteriated derivative.

A comparative study of MFEs with different kinds of high-energy radiation was reported by Klein. ${ }^{169}$ The
MFE is largest with vacuum-UV radiation and smallest with $\alpha$-particles. These results indicate that nongeminate ion recombination contributes more to the total radioluminescence if high ion concentrations are produced with multi-pair spurs and possible overlap of successive spurs in a track as to be expected for $\alpha$-irradiation.

Since spin evolution due to isotropic hyperfine coupling is a coherent process, oscillations should appear in the singlet probability of a geminate radical pair, and hence in the radioluminescence intensity, if there are only a few different coupling constants or if they occur in simple multiple ratios. Such a phenomenon is believed to show up in the radioluminescence of $2 \mathrm{bPPD}$ (31) solutions (cf. Figure 25) investigated by Klein and Voltz. $169,387,388$

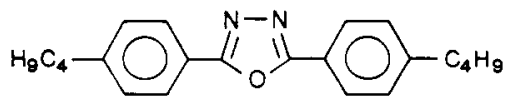

31 (2bPPD)

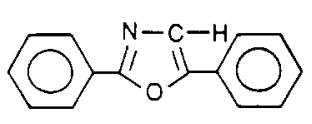

32 (PPO)
Quantum beats, which have been directly related to the hfc constants of the respective radical pairs, have been observed by Anisimov et al. ${ }^{167,386}$ using two-solute systems with perdeuteriated $p$-terphenyl as the cationic species and tetramethylethylene or durene as the anionic species. The quantum beats are clearly resolved, 


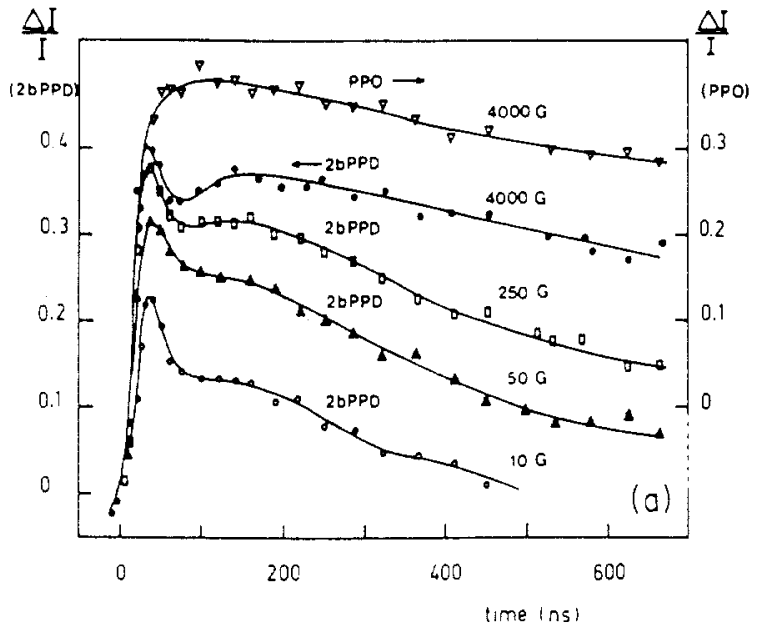

Figure 25. Time dependence of relative $\beta$-ray-excited radioluminescence intensity $\Delta I / I$ of $1.5 \times 10^{-3} \mathrm{M}$ solutions of $2 \mathrm{bPPD}$ (31) and PPO (32) in cyclohexane at room temperature with various magnetic fields. Reprinted from ref 387 with kind permission of R. Voltz; copyright 1977 National Research Council of Canada.

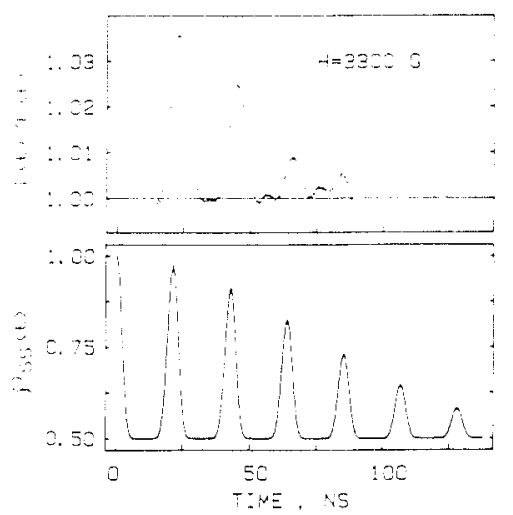

Figure 26. Top: quantum beats evaluated from the time profile of $\beta$-ray-excited scintillation decay from (tetramethylethylene $)^{\circ+} /\left(p \text {-terphenyl- } d_{14}\right)^{\circ-}$ ion pair recombination in transdecalin. Bottom: Theoretical time dependence of the singlet-state population $\left(\rho_{\mathrm{SS}}(t)\right)$. Reprinted from ref 167 with kind permission of Yu. N. Molin; copyright 1983 Elsevier Science Publishers B.V.

and the beat period is in excellent agreement with the theoretical value (cf. Figure 26). The advantage of the systems chosen lies in the fact that in both examples the protons in the anionic species constitute one set of equivalent magnetic nuclei, whereas hyperfine coupling in the perdeuteriated cation is weak. The Fourier transform of the experimental beat curves fits well with the ESR spectra of the radical ions studied.

Another interesting case of quantum beats in radioluminescence has been recently reported by Veselov et al. ${ }^{168}$ Here the radical cation is an S-centered radical cation from diphenyl sulfide, and the radical anion is again perdeuteriated $p$-terphenyl. Singlet-triplet transitions in this radical ion pair are mainly due to the $\Delta g$ mechanism. Thus the beat frequency is proportional to the magnetic field strength. The observation of this effect is another example for spin-memory effects with S-centered radicals, as otherwise found only with photochemically produced sulfonyl radicals in micellar solution by Hayashi et al. 398

In concluding this section, we note that, although the MFEs observed in radioluminescence offer valuable insight into the details of primary radiochemical events, so far no MFE has been reported for irreversible ra-

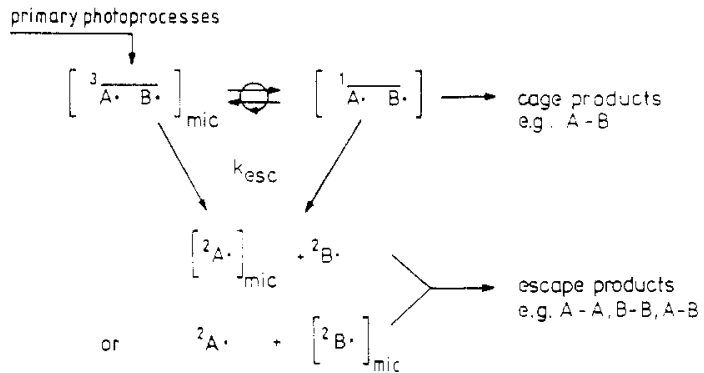

Figure 27. General reaction scheme for radical pairs in micellar cages. [ $]_{\text {mic }}$ denotes micellar solubilization.

diochemical change. Brocklehurst ${ }^{46,399}$ theoretically investigated the situation in aqueous solutions where the most interesting radiochemistry is going on. Here the behavior of neutral radicals as $\mathrm{H}$ atoms and $\mathrm{OH}$ radicals should be of importance, which are, however, less favorable for magnetic field effects, since they show rapid escape due to unhindered diffusion and, as far as the linear $\mathrm{OH}$ radical is concerned, are probably subject to very fast spin relaxation. ${ }^{399,400}$

\section{In Micellar Solution}

\section{General Situation and Methods}

The basic requirement of the radical pair mechanism, easy separability of geminate radical pairs, however with an extended period of diffusive reencounters, seems to be ideally fulfilled in micellar aggregates. Huge enhancements of cage recombination yields as compared to homogeneous solution were first detected by Turro, ${ }^{401}$ who coined the term "micellar supercage effect" ${ }^{47}$ Due to this effect it is no longer necessary that the radicals of the pair are held together by Coulombic forces in order to increase their geminate reencounter probability. In fact, most investigations of magnetic field effects in micellar solution have involved neutral radical pairs produced in reactions of excited carbonyl triplets (cf. Tables 11 and 12). Here one deals with the situation that triplet radical pairs are produced in micellar cages either by hydrogen atom transfer from the detergent or some additional hydrogen donor or by homolytic $\alpha \mathrm{C}-\mathrm{C}$ bond cleavage in aliphatic ketones. The overwhelming part of these investigations has been contributed by the groups of Turro, Hayashi, Tanimoto, and Scaiano (cf. Tables 11-13). Several investigations involving electron-transfer reactions in reverse micellar systems (microemulsions) have been reported by our group (cf. Table 13).

A general mechanistic framework for MFEs in micellar systems is provided in Figure 27. The symbol [ ] $]_{\text {mic }}$ denotes containment of the respective species within a micelle, the bar over the radical pair indicating spin correlation as specified by the superscript. For the systems reviewed in this section radical pair recombination is only possible with formation of diamagnetic product states. Therefore, if the pairs are generated with triplet spin correlation, a multiplicity change has to occur before recombination is possible.

In Figure 27 this ISC process in the radical pair is indicated by equilibrium arrows and a circular arrow distinguishing the contribution of incoherent and coherent processes, respectively (vide infra). Cage product 
TABLE 11. Magnetic Field Effects on Radical Pairs from $\alpha$-Cleavage of Ketones in Micellar Solution

\begin{tabular}{|c|c|c|c|}
\hline ketone $^{a}$ & surfactant ${ }^{b}$ & type of expt $t^{c}$ & ref \\
\hline \multirow[t]{7}{*}{ DBK (33) } & HDTC & ${ }^{13} \mathrm{C}$ enrichment & Turro et al. ${ }^{143,402,404-408,413}$ \\
\hline & & $\mathrm{CP}$, cage effect & Turro et al. ${ }^{119}$ \\
\hline & SDS & LFP, kinetics & $\begin{array}{l}\text { Hayashi et al., } \\
\text { Sakaguchi et al }{ }^{162,424}\end{array}$ \\
\hline & HDTC & LFP, kinetics & $\begin{array}{l}\text { Sakaguchi et al. }{ }^{424} \\
\text { Turro et al. } ._{11,412}\end{array}$ \\
\hline & SDS, HDTC & $\mathrm{CP},{ }^{17} \mathrm{O}$ enrichment & Turro et al. ${ }^{148}$ \\
\hline & SDS & LFP, time-resolved CIDNP & Turro et al. ${ }^{415}$ \\
\hline & SOS, SDeS, SDE & $\begin{array}{l}\text { emulsion polymerization } \\
\mathrm{CP}, B_{0} \text { dependent }{ }^{13} \mathrm{C} \text { CIDNP }\end{array}$ & Turro et al. ${ }^{142,414,418}$ \\
\hline \multirow{3}{*}{$33 \mathbf{a}$} & $\begin{array}{l}\text { HDTC } \\
\text { HDS, SDES SDL }\end{array}$ & CP, cage effect & $\begin{array}{l}\text { Zimmt et al. } \\
\text { Turro et al }{ }^{119,401}\end{array}$ \\
\hline & & $\mathrm{CP},{ }^{1} \mathrm{H}$ CIDNP & Hutton et al. 403 \\
\hline & & $\mathrm{CP}, \mathrm{MFE}$ quenching by $\mathrm{Ln}^{3+}$ ions & Turro et al. ${ }^{420}$ \\
\hline $33 \mathbf{a}-\mathbf{c}$ & HDTC & LFP, kinetics & Turro $^{412}$ \\
\hline $33 \mathrm{c}$ & SDS & emulsion polymerization & Turro et al. ${ }^{414}$ \\
\hline $33 d-f$ & HDTC & $\mathrm{CP}$, cage effect in ultrahigh fields & Turro et al. ${ }^{119}$ \\
\hline $34 \mathrm{c}$ & SOS, SDeS, SDS & $\mathrm{CP}, \mathrm{B}_{0}$-dependent ${ }^{13} \mathrm{C}$-CIDNP & Zimmt et al. ${ }^{417}$ \\
\hline $34 a, b$ & SDS, HDTC & CP, cage effect & Baretz and Turro ${ }^{131}$ \\
\hline $34 \mathbf{b}, \mathbf{e}$ & SDS, HDTC & LFP, kinetics & Gould et al. ${ }^{419}$ \\
\hline $34 \mathbf{b}, \mathbf{d}, \mathbf{e}$ & HDTC & $\mathrm{CP}$, cage effect & \\
\hline $34 \mathbf{b}\left(d_{6}, d_{8}\right), 34 \mathbf{e}\left(d_{2}\right)$ & HDTC & LFP, kinetics & Turro et al. \\
\hline $\begin{array}{l}35 \mathbf{a}\left({ }^{17} \mathrm{O}\right) \\
36 \mathbf{a}\left({ }^{17} \mathrm{O}\right)\end{array}$ & SDS, HDTC & $\mathrm{CP},{ }^{17} \mathrm{O}$ enrichment & Turro et al. ${ }^{148}$ \\
\hline $\begin{array}{l}\mathbf{3 6 \mathbf { a }}\left(d_{0}, d_{3}, d_{6}\right) \\
\mathbf{3 6 \mathbf { b }}, \mathbf{3 6 \mathbf { a }}\left({ }^{13} \mathrm{CO}\right)\end{array}$ & HDTC & $\mathrm{CP}$, quantum yields & Turro and Mattay ${ }^{410}$ \\
\hline $36 \mathrm{a}$ & SDS & CP, cage effect & Gould et al. ${ }^{419}$ \\
\hline $36 \mathbf{a}\left(d_{3}, d_{6}\right)$ & $\begin{array}{l}\text { HDTC, HDTB, SDS, Brij } 35 \\
\text { HDTC }\end{array}$ & $\begin{array}{l}\mathrm{CP} \text {, quantum yields } \\
\mathrm{CP} \text {, ultrahigh fields }\end{array}$ & $\begin{array}{l}\text { Turro and Mattay } \\
\text { Turro et al. }{ }^{119}\end{array}$ \\
\hline $36 c$ & SDS, HDTC & $\mathrm{CP}$, cage effect & Gould et al. ${ }^{419}$ \\
\hline $\mathbf{3 7}, \mathbf{3 7 a}, \mathbf{b}$ & SDS & LFP, time-resolved CIDNP & Turro et al. ${ }^{415}$ \\
\hline 38, 38a,b & SDS & $\mathrm{CP}$, cage effect & Gould et al. ${ }^{416}$ \\
\hline $39,39 a, b(\alpha$ and $\beta)$ & $\begin{array}{l}\text { SDS } \\
\text { SDS }\end{array}$ & triplet-sensitized CP, LFP (zero field) & Gould et al. ${ }^{416}$ \\
\hline 40 & SDS & LFP, yield of radical escape & Hayashi et al. ${ }^{398}$ \\
\hline
\end{tabular}

${ }^{\circ}$ Cf. Chart 1. ' Surfactants: HDTC, hexadecylammonium chloride; SDS, sodium dodecyl sulfate; SOS, sodium octyl sulfate; SDeS, sodium decyl sulfate. ${ }^{c} \mathrm{CP}$, continuous photolysis; LFP, laser flash photolysis.

formation does not necessarily mean regeneration of the starting materials, since fast chemical transformation (i.e., decarbonylation ${ }^{421}$ ) may eventually transform the primary radical pair into a more stable form. Escape of one of the radicals from the micelle terminates the lifetime of the correlated pair and finally leads to stable products, too (escape products), which may, or may not, be different from the cage products.

MFEs have been detected, observing various parameters related to the scheme in Figure 27. In continuous-photolysis (CP) methods either relative yields of cage and escape products ("cage effect" in Tables 11 and 12) or the absolute quantum yields may be used to study MFEs. Of particular interest are magnetic isotope enrichments, which will be reviewed in some detail in section IV.H. Time-resolved methods using laser flash photolysis (LFP) have allowed a direct observation of MFEs on the yield of radical escape and on the kinetics of recombination and escape.

The first time-resolved observations of intramicellar radical pair decay under a magnetic field have been reported by Hayashi et al. ${ }^{161}$ for radical pairs from dibenzyl ketone $\alpha$-cleavage and for radical pairs produced from hydrogen abstraction by benzophenone triplet from SDS molecules in the respective micelles. ${ }^{422}$ In the meantime many observations of this type have followed (cf. Tables 11 and 12). A typical example is shown in Figure 28, adopted from Sakaguchi and Hayashi. ${ }^{425}$ The decay curves may be generally described by a double exponential:

$$
I(t)=I_{\mathrm{f}} \exp \left(-k_{\mathrm{f}} t\right)+I_{\mathrm{s}} \exp \left(-k_{\mathrm{s}} t\right)
$$

where the fast component is typically on the order of

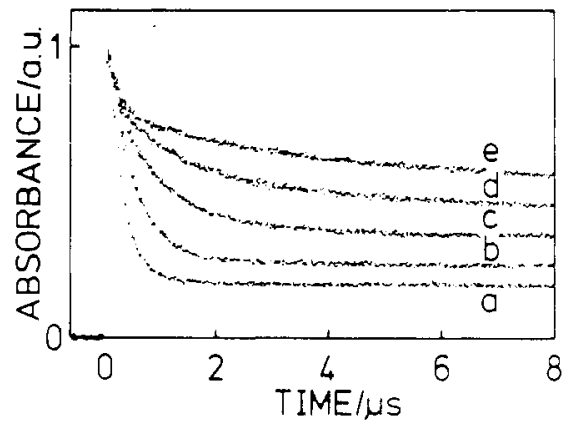

Figure 28. Transient absorbance decay observed at $380 \mathrm{~nm}$ for a micellar SDS solution of methylnaphthoquinone (42a) in various magnetic fields: (a) 0 , (b) 0.03 , (c) 0.1 , (d) 0.3 , and (e) $1.34 \mathrm{~T}$. Reprinted from ref 425 with kind permission of $\mathrm{H}$. Hayashi; copyright 1984 American Chemical Society.

several $10^{6} \mathrm{~s}^{-1}$ and the slow one on the order of $10^{4} \mathrm{~s}^{-1}$ or slower. In some cases two fast-decay components may be distinguished as the magnetic field is increased. ${ }^{419,425,450,767}$ Generally, a MFE is seen during the fast part of the decay. For triplet radical pairs it is slowed down by a factor of up to 10 , as has been reported for the radical pair produced from 4,4'-difluorobenzophenone photolysis in SDS. ${ }^{162}$ Concomitant with the decrease of $k_{\mathrm{f}}$ is a drastic increase in the amplitude of the slow component $I_{\mathrm{s}}$.

The interpretation of these effects is given in terms of the kinetic scheme in Figure 29, where the example of radical production by hydrogen atom transfer has been used. The yield of radical escape, which is obtained from the intensity $I_{\mathrm{S}}$ of the slow kinetic component, is kinetically equivalent to the ratio $k_{\text {esc }} /\left(k_{\text {rec }}\right.$ 
TABLE 12. Magnetic Field Effects on Radical Pairs from H-Atom Abstractions in Micellar Solutions

\begin{tabular}{|c|c|c|c|c|}
\hline $\mathrm{H}$ acceptor ${ }^{a}$ & $\mathrm{H}$ donor ${ }^{b}$ & surfactant ${ }^{c}$ & type of expt & ref \\
\hline benzophenone (41) & $\mathrm{S}$ & SDS, HDTC & $\begin{array}{l}\text { LFP, kinetics } \\
\text { CP, quantum yield } \\
\text { CP, quantum yield of DPBF photooxidation } \\
\text { LFP, kinetics } \\
\text { CIDEP phase anomaly } \\
\text { explanation by }{ }^{3} \text { RP ESR }\end{array}$ & $\begin{array}{l}\text { Sakaguchi et al. }{ }^{422,423} \\
\text { Scaiano et al. } \\
\text { Tanimoto et al. } \\
\text { Sakaguchi et al., } \\
\text { Scaiano et al.443 } \\
\text { Murai et al., } \\
\text { Saki } \\
\text { Closs et al., } \\
\text { Buckley et al. }\end{array}$ \\
\hline & $\begin{array}{l}\mathbf{5 2} \\
\mathrm{S}, \mathbf{5 2} \\
\mathbf{5 2}\end{array}$ & $\begin{array}{l}\text { SDS } \\
\text { CTAC } \\
\text { DODAC vesicles }\end{array}$ & $\begin{array}{l}\text { electron spin echo } \\
\mathrm{LFP} \text {, yield of radical escape } \\
\mathrm{CP} \text { (MF from } \mathrm{Fe}_{3} \mathrm{O}_{4} \text { particles), LFP } \\
\text { (yield of radical escape) }\end{array}$ & $\begin{array}{l}\text { Thurnauer and Meisel }{ }^{447} \\
\text { Scaiano et al. }{ }^{442,443} \\
\text { Fendler, }{ }^{448} \text { Herve et al. }{ }^{114}\end{array}$ \\
\hline $41\left(d_{10}\right), 41\left({ }^{13} \mathrm{C}\right)$ & $\mathbf{S}$ & SDS, CTAC & LFP, kinetics & Sakaguchi et al. ${ }^{423,424}$ \\
\hline $\begin{array}{l}41 \mathrm{c}-\mathrm{e} \\
\text { naphthoquinone }(42)\end{array}$ & $\begin{array}{l}\mathrm{S}, \mathbf{5 2} \\
\mathrm{S}\end{array}$ & $\begin{array}{l}\text { SDS, DTAC } \\
\text { SDS }\end{array}$ & $\begin{array}{l}\text { LFP, yield of radical escape } \\
\text { time-resolved CIDEP } \\
\text { LFP, kinetics } \\
\text { LFP, MFE quenching by } \mathrm{Ln}^{3+} \text { ions }\end{array}$ & $\begin{array}{l}\text { Scaiano and Lougnot } \\
\text { Sakaguchi et al. } \\
\text { Sakaguchi and Hayashi } \\
\text { Sakaguchi and Hayashi } \\
\text { Sa2 }\end{array}$ \\
\hline $\begin{array}{l}42 a \\
\text { acetophenone }(43 a)\end{array}$ & $\begin{array}{l}\mathrm{S} \\
\mathrm{S}, \mathbf{5 3}\end{array}$ & $\begin{array}{l}\text { SDS } \\
\text { SDS } \\
\text { CTAB }\end{array}$ & $\begin{array}{l}\text { LFP, kinetics } \\
\text { LFP, yield of radical escape } \\
\text { LFP, }{ }^{e} \text { kinetics }\end{array}$ & $\begin{array}{l}\text { Sakaguchi and Hayashi }{ }^{42} \\
\text { Hayashi et al. }{ }^{426} \\
\text { Grant et al. }{ }^{121}\end{array}$ \\
\hline $\begin{array}{l}\text { butyrophenones } \\
\mathbf{4 3 b}\end{array}$ & $56 a-f$ & $\begin{array}{l}\text { SDS, CTAC, } \\
\text { CTAB, Brij } 35\end{array}$ & LFP, kinetics & Evans et al. ${ }^{768}$ \\
\hline $43 c$ & $56 c$ & SDS & & \\
\hline xanthone (44) & $\begin{array}{l}\mathbf{5 3} \\
\mathrm{S} \\
\mathbf{5 4} \\
\mathbf{5 5} \\
\mathbf{5 3}, \mathbf{5 3}(d)\end{array}$ & $\begin{array}{l}\text { SDS } \\
\text { SDS } \\
\text { SDS } \\
\text { SDS } \\
\text { SDS }\end{array}$ & $\begin{array}{l}\text { LFP, yield of radical escape } \\
\text { CP, quantum yield } \\
\text { LFP, kinetics } \\
\text { CP, quantum yield; LFP, kinetics } \\
\text { CIDEP, phase anomaly }\end{array}$ & $\begin{array}{l}\text { Hayashi et al. }{ }^{426} \\
\text { Tanimoto et al. }{ }^{435,436} \\
\text { Tanimoto et al. }{ }^{136} \\
\text { Sakaguchi et al. }{ }^{430}\end{array}$ \\
\hline carbenes 45,46 & 52 & $\begin{array}{l}\mathrm{SDS}-\mathrm{O} / \mathrm{W} \\
\text { microemulsion }\end{array}$ & LFP, yield of radical escape & Scaiano and Lougnot ${ }^{164}$ \\
\hline $\begin{array}{l}\text { anthraquinone }(47) \\
\mathbf{4 7}, \mathbf{4 7 a}, \mathrm{b}\end{array}$ & $\mathrm{S}$ & SDS & $\begin{array}{l}\text { TSLE, rise of escape product } \\
\text { LFP, kinetics } \\
\text { CP, quantum yield of bleaching }\end{array}$ & $\begin{array}{l}\text { Tanimoto et al. }{ }^{171} \\
\text { Tanimoto et al. }{ }^{433} \\
\text { Tanimoto et al. }{ }^{151}\end{array}$ \\
\hline
\end{tabular}

${ }^{\circ} \mathrm{Cf}$. Chart 2. ${ }^{\circ} \mathrm{Cf}$. Chart 3; S, surfactant as H-donor. ${ }^{\circ} \mathrm{CTAC}(=\mathrm{HDTC})$, cetyltrimethylammonium chloride; DODAC, dioctadecyldimethylammonium chloride; DTAC, dodecyltrimethylammonium chloride; HDTC (=CTAC), hexadecyltrimethylammonium chloride; SDS, sodium dodecyl sulfate. ${ }^{d} \mathrm{CP}$, continuous photolysis; DBPF, 1,3-diphenylisobenzofuran; LFP, laser flash photolysis; TSLE, two-step laser excitation. ${ }^{e}$ Magnetic difference signal detection; cf. section III. ' $P$ Pobably electron transfer followed by protonation.

TABLE 13. Magnetic Field Effects on Radical Pairs from Photoelectron Transfer in Micellar Systems

\begin{tabular}{|c|c|c|c|c|}
\hline acceptor & donor $^{a}$ & surfactant ${ }^{b}$ & type of expt ${ }^{c}$ & ref \\
\hline duroquinone $\left(T_{1}\right)(48 c)$ & diphenylamine & SDS & LFP, yield of radical escape & Tanimoto et al. ${ }^{434}$ \\
\hline acetophenone $\left(\mathrm{T}_{1}\right)(43)$ & diphenylamine & SDS & LFP, kinetics & Tanimoto et al. ${ }^{438}$ \\
\hline $\begin{array}{l}\text { 2,5-diphenylbenzoquinone } \\
\left(\mathrm{T}_{1}\right)(\mathbf{4 8 h})\end{array}$ & 4-phenylaniline & SDS & LFP, kinetics & $\begin{array}{l}\text { Levin and Kuz'min, }{ }^{449} \\
\text { Levin et al. }{ }^{450}\end{array}$ \\
\hline thionine $\left(\mathrm{T}_{1}\right)(6 \mathrm{~S})$ & aniline & CDBA/benzene $(\mu \mathrm{E})^{b}$ & $\begin{array}{l}\text { LFP, yield of radical escape } \\
\text { CP, bleaching quantum yield }\end{array}$ & $\begin{array}{l}\text { Schlenker et al. }{ }^{439} \\
\text { Schlenker and Steiner }{ }^{152}\end{array}$ \\
\hline & halogenanilines, DMA & $\begin{array}{l}\text { CDBA w/o, var } c_{\mathrm{H}_{2} \mathrm{O}} \\
\text { CDBA/benzene }(\mu \mathrm{E})^{b}\end{array}$ & $\begin{array}{l}\text { LFP, kinetics } \\
\text { LFP, kinetics }\end{array}$ & $\begin{array}{l}\text { Ulrich and Steiner }{ }^{163} \\
\text { Ulrich et al. } 440\end{array}$ \\
\hline oxonine $\left(S_{1}\right)(60)$ & TMPDA & $\mathrm{AOT} /$ isooctane $(\mu \mathrm{E})^{b}$ & LFP, kinetics & Baumann et al. ${ }^{441}$ \\
\hline pyrene $\left(S_{1}\right)$ & DMA & SDS & LFP $^{d}$ (RYDMR), kinetics & Grant et al. ${ }^{121}$ \\
\hline
\end{tabular}

${ }^{a}$ DMA, $N, N$-dimethylaniline; TMPDA, $N, N^{\prime}$-tetramethyl-p-phenylenediamine. ${ }^{b} \mathrm{AOT}$, sodium bis(2-ethylhexyl)sulfosuccinate; CDBA, cetyldimethylbenzylammonium chloride; SDS, sodium dodecyl sulfate; $\mu \mathrm{E}$, water-in-oil microemulsion. ${ }^{c} \mathrm{LFP}$, laser flash photolysis; CP, continuous photolysis. ${ }^{d}$ Magnetic difference signal detection; cf. section III.

$+k_{\text {esc }}$. An explanation of its magnetic field dependence is provided by the magnetic field dependence of $k_{\text {rec }}$, which should be conceived as resulting from a spinsubstate-resolved kinetic scheme $e^{427,440}$ (cf. Figure 30). Here the actual recombination step is preceded by intersystem crossing from the triplet to the singlet pair spin state. The Zeeman splitting of the triplet levels more and more suppresses spin transitions from the outer triplet levels, thus reducing the ISC efficiency and with it the overall rate constant of recombination.
However, whereas in most work published before 1984 it was generally assumed that the multiplicity change is rate determining (i.e., $k_{\mathrm{ISC}}=k_{\text {rec }}$ ) even in zero field, Hayashi and co-workers ${ }^{162,425,427}$ and Steiner and coworkers ${ }^{163,440}$ have pointed out that in most cases this would not be in accord with the absolute value of the hyperfine coupling (typically below $5 \mathrm{mT}$ ) and the $B_{1 / 2}$ values of the MFD observed (typically on the order of 10-30 mT). Actually, in zero field the radical spin state may be close to spin equilibrium, and factors other than 


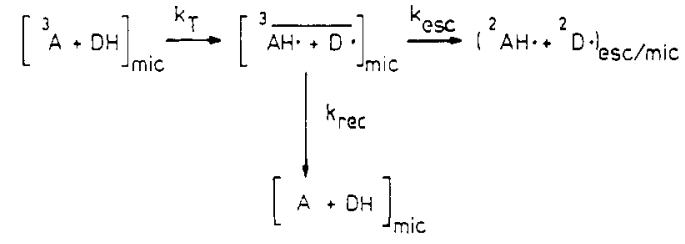

Figure 29. Kinetic scheme for analyzing experimental decay curves of triplet radical pairs in micellar solutions.

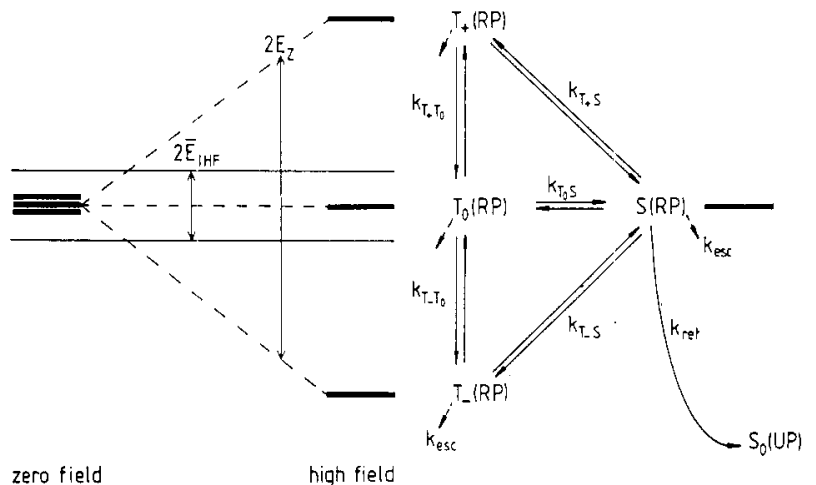

Figure 30. Reaction scheme describing the kinetic relations between spin substates of the radical pair (RP) and the unreacted pair (UP) formed upon recombination. The short dashed arrows indicate the reaction channel of micellar escape, which is equally available for all spin substates. On the left-hand side is shown the energy level scheme for the triplet substates in zero magnetic field and in high field. $E_{\mathrm{IHF}}$ denotes an average hyperfine coupling energy; $E_{Z}$ is the Zeeman energy (from Ulrich, Schlenker, and Steiner ${ }^{440}$ ).

spin ones, e.g., diffusion, may limit the rate constant of recombination.

It should be briefly mentioned that magnetic resonance techniques have also revealed some interesting features specific to radical pairs caged in micelles. Investigating the MFD of ${ }^{13} \mathrm{C}$-CIDNP intensities during photolysis of DBK derivatives in micelles, Zimmt et al. ${ }^{417}$ found that it passes through a maximum at fields between $27.5 \mathrm{mT}$ (in SDS micelles) and $36.5 \mathrm{mT}$ (in SOS micelles). This behavior is similar to that of covalently linked biradicals and demonstrates the influence of the exchange interaction, which decreases as the size of the micelles is increased.

Trifunac and Nelson ${ }^{451}$ observed CIDEP spectra during pulse radiolysis of solutions containing increasing concentrations of surfactant. After reaching the critical micellar concentration, the signals showed polarization patterns attributed to the $\mathrm{S}-\mathrm{T}_{-}$crossing mechanism typical for radical pairs that are forced to remain together wihin a limited separation distance. These results might be reconsidered in the light of intriguing recent findings initiated by a phenomenon first observed by Hayashi and co-workers. ${ }^{429-431}$ They found that the hyperfine lines show an anomalous $\mathrm{E} / \mathrm{A}$ phase pattern, not explicable in terms of current CIDEP mechanisms. The original explanation ${ }^{431}$ attempting to relate this effect to rapid migration of nuclear-spinpolarized $\mathrm{H}$ atoms was made obsolete by a more convincing mechanism suggested by Closs et al..$^{445}$ and Buckley et al. ${ }^{446}$ According to these authors, the anomaly, consisting of an E/A splitting of individual radical hyperfine lines, is readily explained as a consequence of exchange interaction in the caged radical pairs. The "anomalous" CIDEP spectrum observed during the first microseconds is in fact a radical pair
CHART 1. Ketones Investigated That Undergo $\alpha$-Cleavage
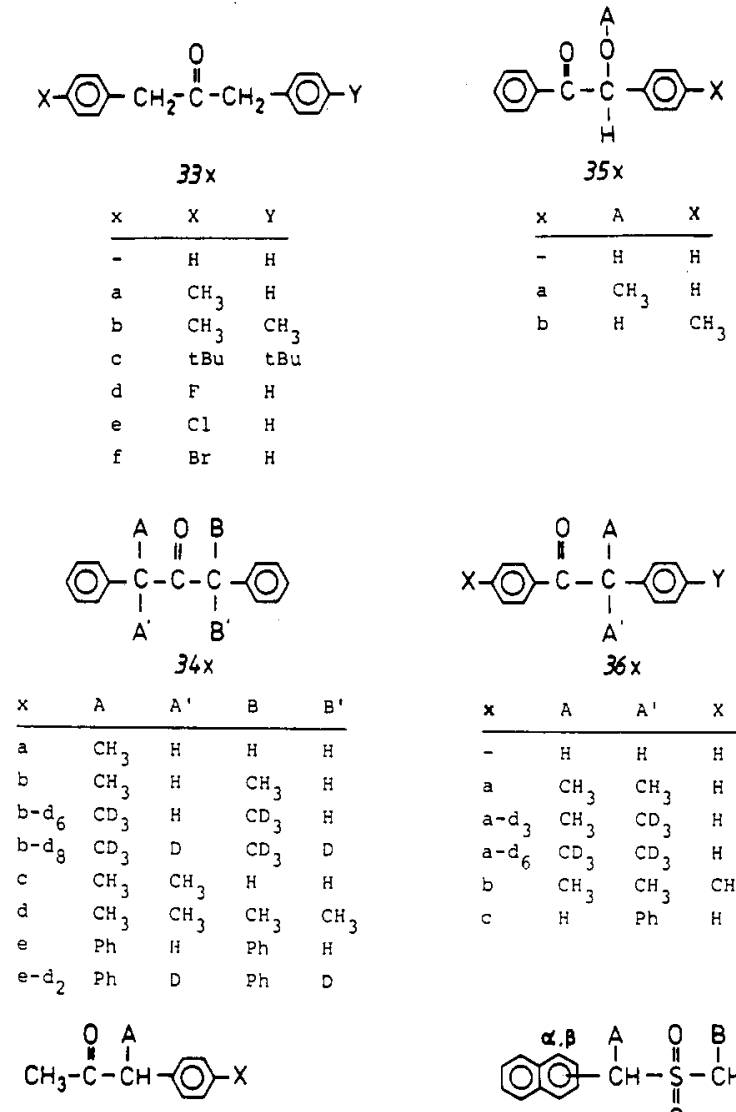

$36 x$

\begin{tabular}{lllll}
$x$ & $\mathrm{~A}$ & $\mathrm{~A}^{\prime}$ & $\mathrm{X}$ & $\mathrm{Y}$ \\
\hline- & $\mathrm{H}$ & $\mathrm{H}$ & $\mathrm{H}$ & $\mathrm{H}$ \\
$\mathrm{a}$ & $\mathrm{CH}_{3}$ & $\mathrm{CH}_{3}$ & $\mathrm{H}$ & $\mathrm{H}$ \\
$\mathrm{a}-\mathrm{d}_{3}$ & $\mathrm{CH}_{3}$ & $\mathrm{CD}_{3}$ & $\mathrm{H}$ & $\mathrm{H}$ \\
$\mathrm{a}-\mathrm{d}_{6}$ & $\mathrm{CD}_{3}$ & $\mathrm{CD}_{3}$ & $\mathrm{H}$ & $\mathrm{H}$ \\
$\mathrm{b}$ & $\mathrm{CH}_{3}$ & $\mathrm{CH}_{3}$ & $\mathrm{CH}_{3}$ & $\mathrm{tBu}$ \\
$\mathrm{C}$ & $\mathrm{H}$ & $\mathrm{Ph}$ & $\mathrm{H}$ & $\mathrm{H}$
\end{tabular}
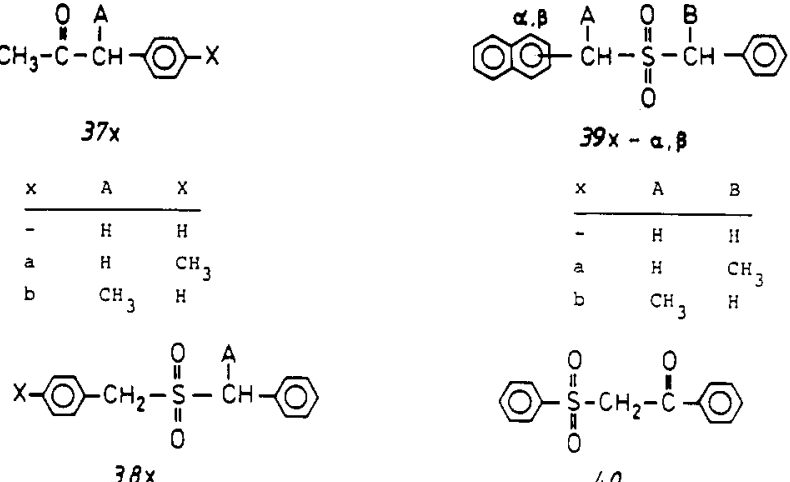

$39 x-\alpha, \beta$

\begin{tabular}{lll}
$\mathrm{x}$ & $\mathrm{A}$ & $\mathrm{B}$ \\
\hline- & $\mathrm{H}$ & $\mathrm{H}$ \\
$\mathrm{a}$ & $\mathrm{H}$ & $\mathrm{CH}_{3}$ \\
$\mathrm{~b}$ & $\mathrm{CH}_{3}$ & $\mathrm{H}$
\end{tabular}
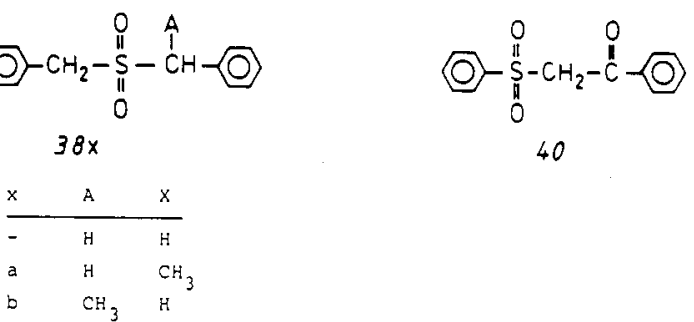

spectrum and converts to a normal CIDEP spectrum of individual radicals as the pair decays due to radical escape from the micelle. As demonstrated by Buckley et al., ${ }^{446}$ this effect can be also observed in highly viscous solvents. One should note, however, that the range of exchange interaction probed by CIDNP and by "anomalous RP-CIDEP" is of largely different order of magnitude (in the latter case only several tenths of a millitesla).

\section{Radical Pairs from $\alpha$-Cleavage of Ketones}

Many examples of MFEs on reactions involving $\alpha$ cleavage of ketones have been reported. A survey of these is presented in Table 11 and Chart 1. Dibenzyl ketone (DBK (33)) and its derivatives are the most examined in the class of compounds susceptible to this type of reaction. The relevant reactions in the photolysis of DBK in micellar solution are described in Figure 31, adopted from Turro's work. ${ }^{404}$ The primary radical 


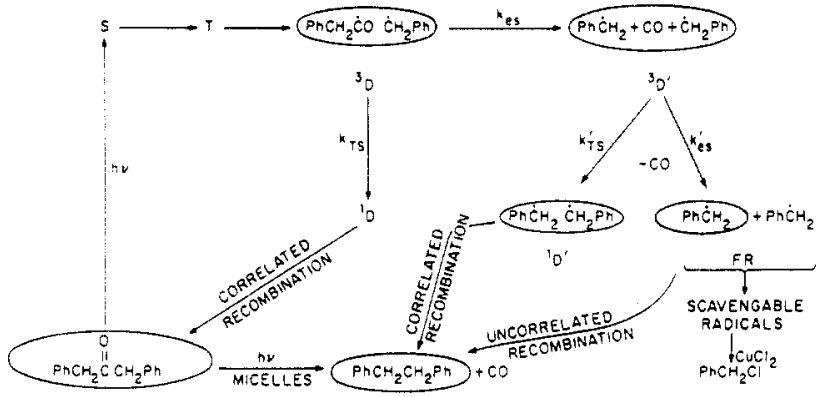

Figure 31. Reaction pathways for photolysis of dibenzyl ketone in micellar solutions. Reprinted from ref 404 with kind permission of N. J. Turro, Columbia University, New York; copyright 1979 American Chemical Society.

pair formed with triplet spin correlation by the photoreaction of the carbonyl triplet comprises a benzyl and a phenylacetyl radical. After a fast change of multiplicity, these may recombine to the starting material or to the isomeric compound 1-phenyl- $p$-methylacetophenone (PMAP). However, by decarbonylation of the phenylacetyl radical, the primary radical pair may also undergo irreversible transformation to a secondary pair. Depending on the stability of the secondary radical thus produced, decarbonylation takes place with rate constants between $6.4 \times 10^{6} \mathrm{~s}^{-1}\left(\mathrm{PhCH}_{2} \mathrm{CO}\right)$ and $1.3 \times 10^{8}$ $\mathrm{s}^{-1}\left((\mathrm{Ph})_{2} \mathrm{CHCO}\right){ }^{421}$ Elimination of $\mathrm{CO}$ does not destroy the spin correlation. The correlated pair of two benzyl radicals present after fast decarbonylation may undergo intramicellar recombination, too, or else may separate by radical escape from the micelle. The extramicellar radicals may be scavenged in the water phase (e.g., by $\mathrm{Cu}^{2+}$ ions ${ }^{401,407}$ or by Fremy's salt ${ }^{415}$ ) or may react to recombination products. In the case of unsymmetrical radical pairs symmetric coupling products will be formed besides the unsymmetric ones. Thus for unsymmetric dibenzyl ketones the product distribution allows the fraction of cage recombination (cage effect) ${ }^{401}$ to be evaluated.

Most of the work described by Turro with the ketones listed in Table 11 employed continuous photolysis combined with chromatographic product distribution analysis. It was found that the fraction of correlated intramicellar recombination not only was magnetic field dependent but also was sensitive to ${ }^{13} \mathrm{C}$ or ${ }^{2} \mathrm{H}$ magnetic isotope effects (cf. Table 11). These will be separately reviewed in section IV.H.

In the case of symmetrical dibenzyl ketones the identity of cage and escape recombination product demands different methods to assess the micellar cage effect. Useful indicators are provided, e.g., by the quantum yield of DBK decomposition or by the effect of scavengers. A more direct method, however, is time-resolved observation of the intramicellar radical kinetics by laser flash photolysis. The method has been applied to $\alpha$-cleavage reactions by Hayashi and coworkers ${ }^{161,162,424}$ and by Turro and co-workers. ${ }^{411,412} \mathrm{~A}$ very detailed investigation involving several ketones of type 34 and 36 has been reported recently by Gould et al. ${ }^{419}$ In Figure 32 is shown the MFD of the fast rate constant of radical decay for two of these compounds. A saturation is not reached below $80 \mathrm{mT} . B_{1 / 2}$ values are approximately $10 \mathrm{mT}$, whereas the characteristic hyperfine fields (from eq 43 ) are 1.5 and $3.1 \mathrm{mT}$ for $34 \mathrm{e}$ and $34 \mathrm{~b}$, respectively.
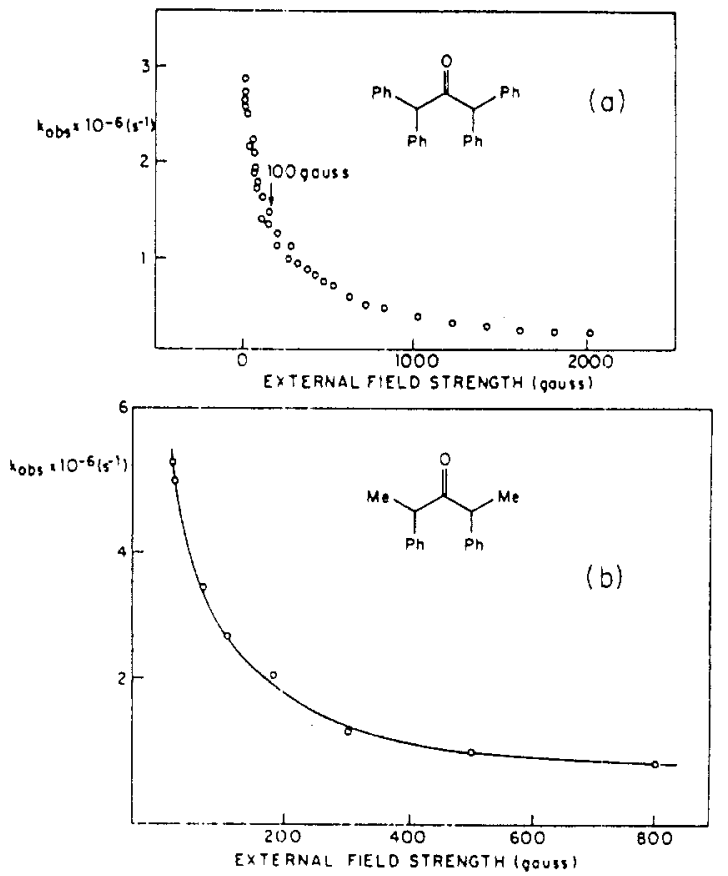

Figure 32. Plot of MFD of rate constant $\left(k_{\text {obsd }}\right)$ of radical pair decay in HDTC micellar solution: (a) ketone 34e; (b) ketone 34b. Reprinted from ref 419 with kind permission of N. J. Turro, Columbia University, New York; copyright 1985 American Chemical Society.

Recently, the magnetokinetic effect of $\mathrm{H} / \mathrm{D}$ magnetic isotope substitution in ketone $\mathbf{3 4}$ (cf. Table 11) was studied by Turro et al. ${ }^{767}$ There is a small magnetic isotope effect at zero field, demonstrating that in HDTC micelles the hyperfine-induced $\mathrm{T} \rightarrow \mathrm{S}$ process is at least of the same order of magnitude as the diffusion-controlled intramicellar reencounter process. The magnetic isotope effect increases with the magnetic field, however, reaching a saturation limit at about $10 \mathrm{mT}$, although in this field region the absolute rate constants of recombination are still strongly magnetic field dependent. This behavior is explicable in terms of the relaxation mechanism as far as it is due to the stochastic modulation of anistropic hyperfine coupling by molecular motion.

An interesting practical application of the micellar cage effect and MFE has been reported by Turro et al. ${ }^{142,414,418}$ Emulsion polymerization of styrene and methyl methacrylate photosensitized by dibenzyl ketones was markedly influenced by an external magnetic field. Since the initial state of such polymerizations starts with monomers solubilized in micelles, it is favorable that initiator radical pairs produced there undergo efficient escape in order to avoid fast intramicellar recombination with termination of the chain reaction. Since the amount of escape for triplet radical pairs increases in a magnetic field, higher yield and higher molecular weights can be obtained under such conditions. ${ }^{13} \mathrm{C}$ magnetic isotope effects, which have also been observed can be understood by the same reasoning.

The importance of spin correlation effects for these phenomena has been demonstrated by using diphenylazoethane $\left(\mathrm{PhCH}_{3} \mathrm{CH}-\mathrm{N}=\mathrm{N}-\mathrm{CHCH}_{3} \mathrm{Ph}\right)$ as an initiator of polymerization. Three modes of radical generation have been compared, viz., thermal, direct photochemical, and triplet-sensitized photochemical. ${ }^{414}$ 


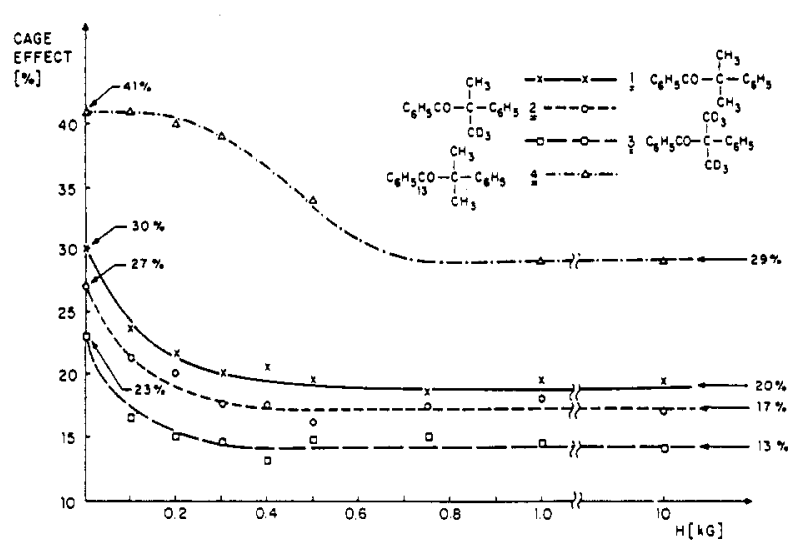

Figure 33. MFD of the cage effect in continuous photolysis experiments with ketones 36 in HDTC micellar solution. Reprinted from ref 410 with kind permission of $\mathrm{N}$. J. Turro, Columbia University, New York; copyright 1981 American Chemical Society.

Only in the last case was a MFE observed, indicating that only the kinetics of initially triplet spin correlated pairs is significantly influenced by a magnetic field.

A MFE on methyl methacrylate polymerization in a three-phase system has also been reported by Imoto and Nomoto. ${ }^{453}$ The mechanism in this case is, however, less obvious than in Turro's system. Other compounds showing a photochemical behavior similar to that described in Figure 31 are the deoxybenzoins 36, the methyl benzyl ketones 37 , and the sulfones 38 and 39 .

For the photodecomposition of deoxybenzoins the MFD of micellar cage effects has been investigated in some detail by Turro and Mattay. ${ }^{410}$ In this case $\alpha$ cleavage produces a triplet-spin-correlated pair of a benzoyl and a substituted benzyl radical. Cage products are the starting materials and the disproportionation products 58 and 59 or their respective deuteriated

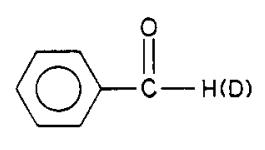

58

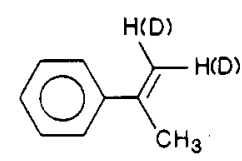

69 analogues. The observed MFD of the cage effect is depicted in Figure 33. The decrease in cage recombination yield must be attributed to the suppression of triplet-singlet transition in the radical pair due to the magnetic field. The half-field value is about $10 \mathrm{mT}$ for compound 36a and its deuteriated derivations $36 \mathbf{a}-d_{3}$ and $36 \mathrm{a}-d_{6}$. ${ }^{13} \mathrm{C}$-Substitution of the carbonyl $\mathrm{C}$ atom leads to a marked high-field shift of the half-field value to about $50 \mathrm{mT}$. While this effect seems to reflect the larger hyperfine constant $(12.8 \mathrm{mT})$ of ${ }^{13} \mathrm{CO}$ in the benzoyl radical, the relation between hyperfine coupling constant and half-field values does not follow the relation established for homogeneous solution, ${ }^{306}$ however. The deoxybenzoins 36 and several halogen-substituted dibenzyl ketones 33d-f have been investigated under conditions of very high magnetic fields up to $14.5 \mathrm{~T} .{ }^{119}$ For the chlorine compound 33e the cage effect, after first decreasing at low fields like in the other cases, increases at high field, which may be attributed to the $\Delta g$ mechanism (the chlorine substituent causes a $\Delta g$ of about $5.5 \times 10^{-4}$ in the radical pair). For the bromine derivative the MFE is completely quenched, which indicates the introduction of efficient, magnetic-field-in- dependent spin relaxation pathways. This aspect has been investigated in more detail by Ulrich et al. ${ }^{440}$ with radical pairs from triplet electron-transfer reactions in microemulsions (vide infra).

In the sulfones 38 and 39 the $\mathrm{SO}_{2}$ group adopts the role of $-\mathrm{CO}$ - in the ketones. ${ }^{416}$ The photochemical products are derived from radical recombination (cage and escape) after $\alpha$-cleavage and fast $\mathrm{SO}_{2}$ elimination $\left(k>10^{8} \mathrm{~s}^{-1}\right)$. The sulfones 38 and $39 \alpha$ apparently react via their triplet state. Both direct photolysis and triplet-sensitized photolysis lead to $80 \%$ yield of cage recombination, which is decreased by a factor of 0.5 in a magnetic field of $0.3 \mathrm{~T}$. With the $\beta$-naphthyl-substituted sulfones 39 there is $100 \%$ cage recombination with no MFE in direct photolysis but $90 \%$ cage product formation with a magnetic field effect of $-40 \%$ in triplet-sensitized photolysis. The latter result indicates that for $39 \beta$ direct photolysis leads to singlet-spincorrelated radical pairs that recombine very efficiently and, obviously, have no chance for a crossover to the triplet state. In contrast to the sulfones 38 and 39 undergoing rapid $\mathrm{SO}_{2}$ elimination after $\alpha$-cleavage, the sulfone 40 investigated by Hayashi et al. ${ }^{398}$ must be assumed to decompose into a radical pair $\left(\mathrm{PhCOCH}_{2}{ }^{\circ}\right.$ $+\mathrm{PhSO}_{2}{ }^{\circ}$ ) where the phenylsulfonyl radical does not eliminate $\mathrm{SO}_{2}{ }^{452}$ so that a MFE with an S-centered radical may be studied. Using SDS micellar solution it was found ${ }^{398}$ that the yield of radical escape detected by laser flash photolysis increased by $53 \%$ in a magnetic field of $1.2 \mathrm{~T}$. This MFE indicates that the radical pairs were formed from a triplet precursor and, what may be of importance for magnetic isotope separation of ${ }^{33} \mathrm{~S}$, that spin relaxation in S-centered radicals does not too rapidly destroy a spin memory in the radical pair (cf. also section C.4 and ref 168 ).

\section{Radical Pairs from H-Atom-Abstraction Reactions}

A survey of MFE investigations with this type of reaction is provided in Table 12 .

Using laser flash spectroscopy, Hayashi and his collaborators have intensively investigated reactions with benzophenone triplet in anionic (SDS) and cationic (CTAC, DTAC) micelles. ${ }^{162,422-424,429}$ The kinetic effects observed are similar to those observed with radical pairs from $\alpha$-cleavage. Whereas in Hayashi's work the radical pairs were produced by $\mathrm{H}$-atom abstraction from surfactant molecules, Scaiano and co-workers ${ }^{442,443}$ investigated the reaction with 1,4-cyclohexadiene (52) as a hydrogen donor as well. From the magnetic-field-dependent kinetics a rate constant of $5.8 \times 10^{6} \mathrm{~s}^{-1}$ was evaluated for the escape of the cyclohexadiene radical from SDS micelles. ${ }^{442}$ A similar value was obtained with a novel spin-echo technique by Thurnauer and Meisel. 447

A very interesting variation of a MFE with the benzophenone/cyclohexadiene system has been reported by Fendler and co-workers. ${ }^{114,448}$ The reaction was carried out in DODAC vesicles including $\mathrm{Fe}_{3} \mathrm{O}_{4}$ particles. Variation of the concentration of these particles was shown to be equivalent to the variation of an external magnetic field. The fraction of escape radicals could be increased from $55 \%$ in the absence of $\mathrm{Fe}_{3} \mathrm{O}_{4}$ to a maximum of $86 \%$ with $\mathrm{Fe}_{3} \mathrm{O}_{4}$ present. This value corresponds to a complete blocking of $T_{+}$and $T_{-}$radical pair states from intersystem crossing. 
CHART 2. Carbonyl Compounds Investigated as Hydrogen Acceptors
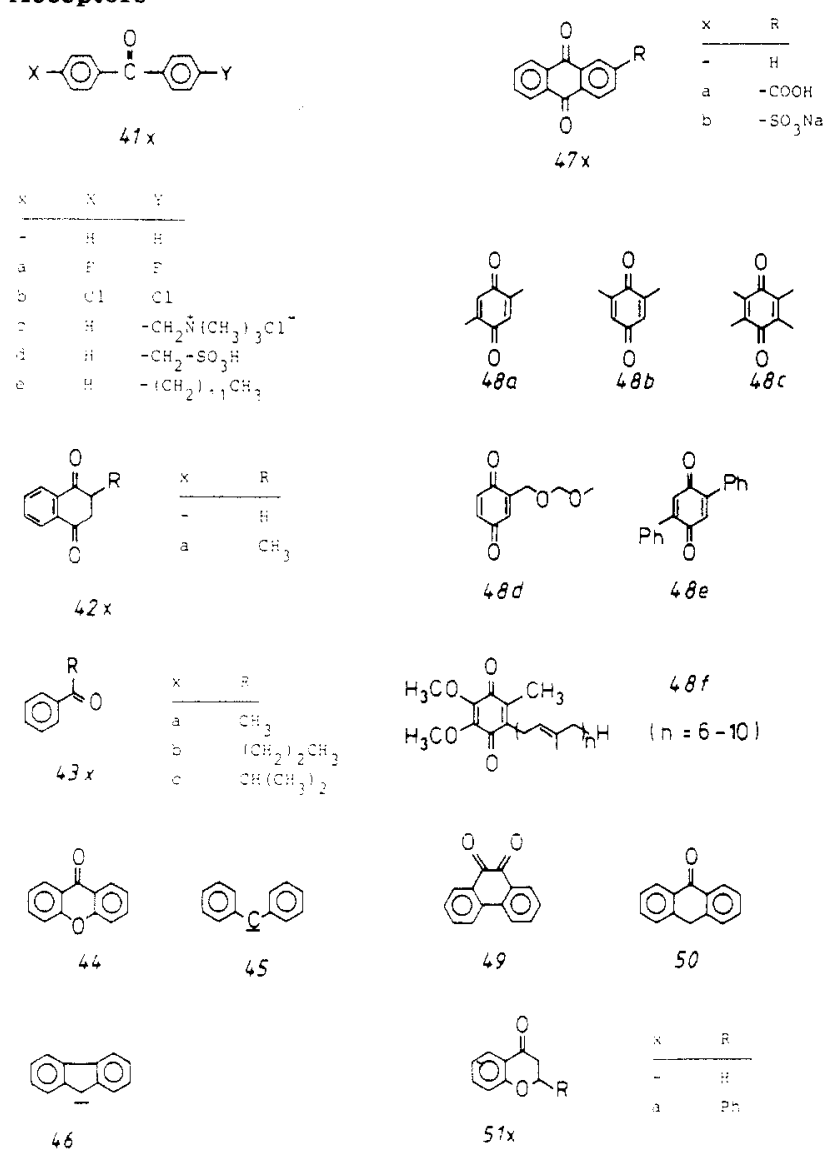

480
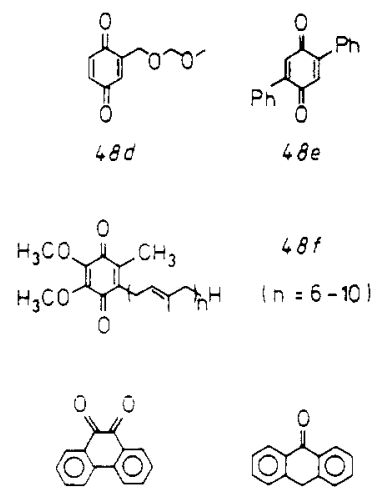

49

50

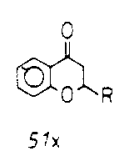

Triethylgermanium hydride (53) was used as a hydrogen donor by Sakaguchi and Hayashi ${ }^{425}$ to produce radical pairs in the reaction with xanthone (44). A marked MFE of $+24 \%$ at $1 \mathrm{~T}$ was observed on the yield of escape radicals, demonstrating that spin relaxation in the germanium-centered radical is not strong enough to suppress the magnetic field effect on the radical pair intersystem crossing. Thus experiments with magnetic isotopes of germanium can be expected to exhibit a magnetic isotope effect, possibly allowing ${ }^{73} \mathrm{Ge}$ isotope enrichment.

Tanimoto et al. ${ }^{136,435,436}$ used xanthene (54) and 9,10-dihydroanthracene (55) as hydrogen donors for the reaction with xanthone triplet. For these systems acceptor and donor radicals could be observed by laser flash photolysis. The MFE is similar to that of other micellar systems, the yield of escape radicals being increased, however, by a factor of 4 in a magnetic field of $0.3 \mathrm{~T}$ with a $B_{1 / 2}$ value of about $20 \mathrm{mT}$.

Intramicellar reactions with quinones have also been investigated by Tanimoto and collaborators (cf. Table 12). A variety of experimental methods have been used, viz., stationary photolysis, combined with direct UV analysis or chemical analysis, and time-resolved measurements by laser flash photolysis and two-step laser excitation (cf. also section III). The MFEs found are in good agreement with the general behavior described for micellar systems. An interesting photochemical application has been described ${ }^{150}$ where the radical pairs produced by the reaction of anthraquinone triplet or benzophenone triplet with SDS are used to sensitize the oxygenation of diphenylbenzofuran (DPBF). It could be shown that the quantum yield of DPBF disappear-
CHART 3. Hydrogen Donors

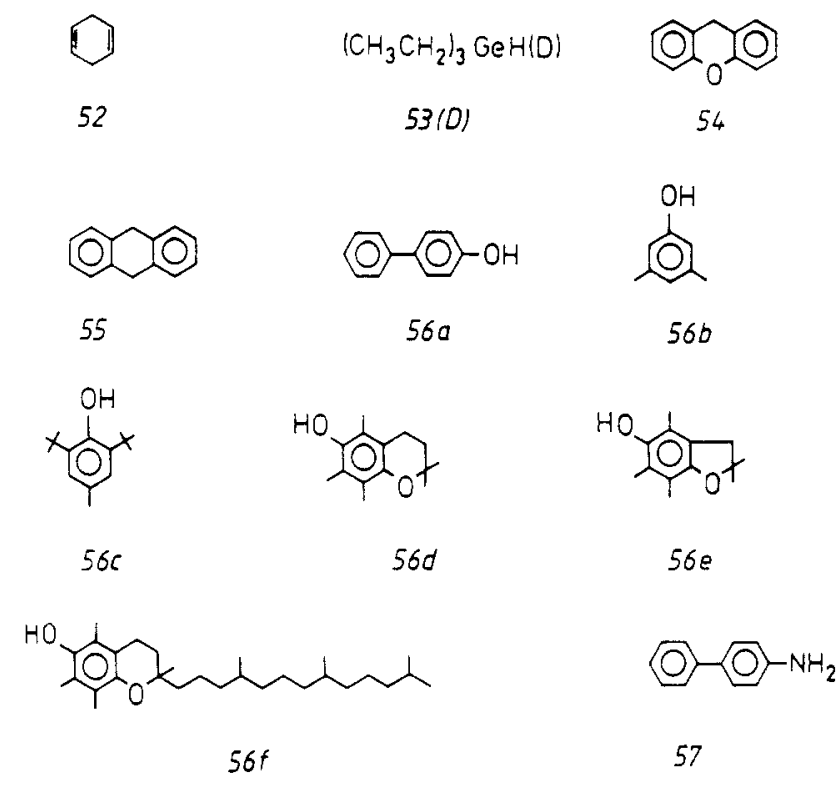

ance is increased by $180 \%$ in a magnetic field of 0.15 $\mathrm{T}, B_{1 / 2}$ ranging between 30 and $40 \mathrm{mT}$.

A time-resolved study of magnetic-field-dependent intramicellar radical pair recombination kinetics involving radicals from $\mathrm{H}$-atom transfer between $p$ phenylphenol (56) and 2,5-diphenylbenzoquinone (52) triplet has been reported by Levin et al. ${ }^{449,450}$ These authors measured the MFE on the recombination rate constant at various temperatures, from which the activation energy was obtained as $25 \mathrm{~kJ} / \mathrm{mol}$ in zero field and $12.5 \mathrm{~kJ} / \mathrm{mol}$ at $0.35 \mathrm{~T}$. These values have been associated with activation of translational and rotational diffusion, respectively. The authors suggested that the decrease with the magnetic field might be attributed to the circumstance that the relaxational transitions responsible for the radical pair recombination kinetics in strong fields are a function of the radicals' rotational mobility, the activation energy of which is lower than for translational diffusion, which is thought to be rate determining in zero field.

Examples of MFEs on intramicellar recombination kinetics with ketyl-aryloxy radical pairs from the photoreaction between butyrophenones $43 \mathrm{~b}, \mathrm{c}$ and the phenols 56b-f in various micellar systems have been recently reported by Evans et al. ${ }^{768}$ The radical pairs involving phenoxyl radicals were found to behave very similarly to the analogous carbon-centered radicals.

MFEs have also been found for carbene reactions in water-in-oil (w/o) microemulsions. Scaiano and Lougnot $^{164}$ generated carbenes 45 and 46 by photolysis of diphenyldiazomethane or diazofluorene. Radical pairs, originating by hydrogen abstraction of these carbenes from 1,4-cyclohexadiene, showed the typical MFE for triplet radical pairs, demonstrating that triplet carbenes were the precursors of the radical pairs.

An interesting study of the influence of $\mathrm{Ln}^{3+}$ ions on the intramicellar radical decay kinetics of radical pairs involving naphthosemiquinone has been reported by Sakaguchi and Hayashi. ${ }^{428}$ These ions do not influence the zero-field kinetics but quench the magnetic field sensitivity as their concentration is increased. This effect may be understood as an increase of the $T_{ \pm} \rightarrow$ $\mathrm{T}_{0}, \mathrm{~S}$ relaxation rate by magnetic perturbations due to 
the $\mathrm{Ln}^{3+}$ ions. However, the capability of quenching seems to correlate with the electron spin quantum number of the ions, rather than with the effective magnetic moment, which also receives considerable contributions from orbital angular momentum. The quenching efficiency is at maximum for $\mathrm{Gd}^{3+}(S=7 / 2$, $L=0, \mu_{\text {eff }}=7.94 \mu_{\mathrm{B}}$ ). The ions $\mathrm{Dy}^{3+}$ and $\mathrm{Ho}^{3+}$, which have higher total magnetic moments but smaller spin quantum numbers, are less efficient. Turro et al. ${ }^{420}$ performed a similar investigation, studying the sensitivity of the magnetic-field-dependent cage effect in $p$-Me-DBK (33a) photolysis to $\mathrm{Ln}^{3+}$ ions. Applying the complete series of $\mathrm{Ln}^{3+}$ ions (of course with the exception of $\mathrm{Pm}^{3+}$ ), their results are generally in line with those of Sakaguchi and Hayashi. However, as a very important additional detail they reported a nil effect for $\mathrm{Eu}^{3+}$ (which had not been included by the Japanese authors). Since $\mathrm{Eu}^{3+}$ has a high spin quantum number $(S=3)$, this ion is an exception to the apparent rule that angular momentum contributions are not involved in the quenching of the MFE.

\section{Radical Pairs from Photo-Electron-Transfer Reactions}

Examples of MFE studies with radical pairs from photoelectron transfer in micelles or related systems are less numerous than with radical pairs from the typical carbonyl triplet reactions discussed above. They are listed in Table 13.

Carbonyl compounds have been used as electron acceptors in normal micelles by Tanimoto et al. ${ }^{434,438}$ and by Levin et al. ${ }^{449,450}$ Whereas a MFE on the radicals produced by electron transfer from diphenylamine to duroquinone (48c) in SDS micelles was hardly detectable, ${ }^{434}$ a better resolution of a MFE could be obtained when the radicals were produced with 1-acetonaphthone triplet as an electron acceptor. ${ }^{438}$ The yield of long-lived radicals was increased by a magnetic field, and the intramicellar radical pair recombination, occurring with a rate constant of $7 \times 10^{6} \mathrm{~s}^{-1}$ in zero field, was slowed down to $7 \times 10^{5} \mathrm{~s}^{-1}$ in a field of $0.5 \mathrm{~T}$. The radicals resulting from the photoreaction of 2,5-diphenylbenzoquinone (48e) with $p$-phenylaniline, the magnetic-field-dependent kinetics of which has been observed by Levin and Kuz'min ${ }^{449}$ (cf. last section), are probably also due to an electron-transfer reaction. A MFE is observed, however, only at a $\mathrm{pH}$ where the semiquinone radical anion becomes protonated. In alkaline solutions the electron-transfer products are assumed to remain associated and to decay as a triplet exciplex $\left(k \approx 10^{7}\right.$ $\mathrm{s}^{-1}$ ), with no MFE on the decay kinetics. In fact, the decay is too slow to expect MFEs according to the triplet mechanism as observed for heavy-atom-substituted exciplexes by Steiner et al. ${ }^{80,160}$

Water-in-oil microemulsions, where aqueous nanodroplets $^{454}$ are surrounded by a closed surfactant monolayer, have been used by Steiner and co-workers ${ }^{152,163,439,440,441}$ to study electron-transfer-generated radical pairs from thionine triplet $(6 \mathrm{~S})$ and aniline derivatives. MFEs have been studied with variation of the radius of the nanodroplets $(1.3-5.2 \mathrm{~nm}) .{ }^{163}$ Whereas the rate constant of escape was proportional to $r^{-1}$, the rate constant of rerombination varied as $r^{-3}$. Only the latter was found to be magnetic field dependent. It is decreased by a factor of $2-3$ when the field is increased

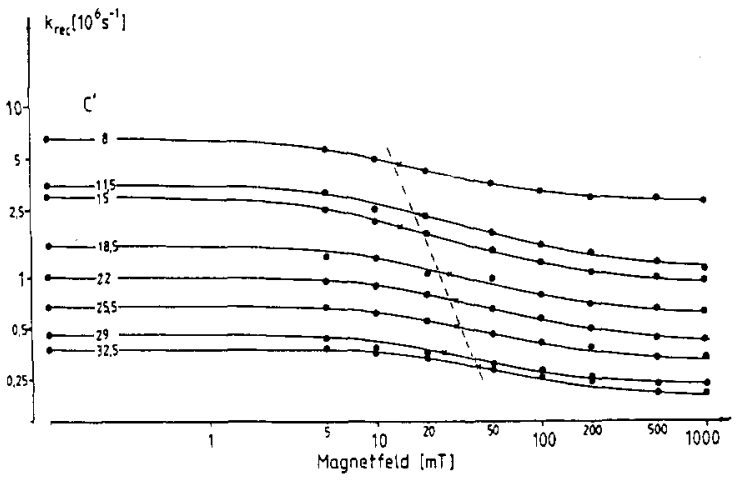

Figure 34. MFD of the geminate recombination rate constant of (thionine $)^{*} /(\text { aniline })^{++}$radical pairs produced by thionine triplet quenching in CDBA/benzene $/ \mathrm{H}_{2} \mathrm{O}$ microemulsions of variable water content $\left(c^{\prime}=\left[\mathrm{H}_{2} \mathrm{O}\right] /[\mathrm{CDBA}]\right)$ : (๑) experimental points; $(X)$ interpolated $B_{1 / 2}$ positions (from Ulrich and Steiner ${ }^{163}$ ).

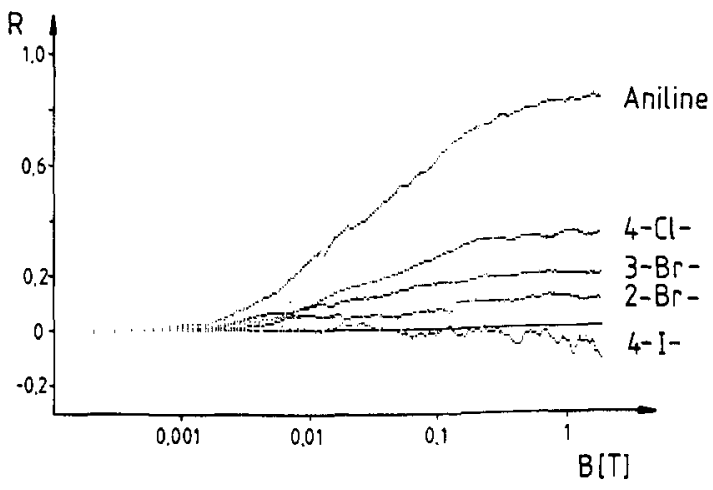

Figure 35. Relative MFE $(R)$ on the free radical yield produced in thionine triplet quenching with various anilines and monitored $20 \mu$ s after the laser pulse. The magnetic field was scanned continuously over 480 pulses. Each data point represents a floating average of 30 pulses.

from zero field to $1 \mathrm{~T}$. The $B_{1 / 2}$ values showed a significant increase from 14 to $40 \mathrm{mT}$, with the nanodroplet radius increasing from 1.3 to $5.2 \mathrm{~nm}$ (cf. Figure 34). These results have provided evidence that radical pair reencounters are rate determining for recombination at zero field (decreasing as $r^{-3}$ as the radius of the nanodroplets increases) whereas higher and higher magnetic fields are necessary to make spin relaxation $\mathrm{T}_{+1-} \rightarrow \mathrm{T}_{0}, \mathrm{~S}$ slow enough as to show up in the overall geminate recombination kinetics.

This interpretation is also corroborated by heavyatom effects investigated with halogen-substituted anilines in the same system. ${ }^{440}$ Figure 35 shows the MFD of the yield of radical escape with various electron donors. The MFE is systematically reduced as the spinorbit coupling strength increases. With 4-iodoaniline there is even a sign inversion of the effect, which has been attributed to the triplet mechanism. Time-resolved measurements ${ }^{440}$ have shown that the heavyatom effects do not significantly influence the zero-field radical pair recombination kinetics but reduce their magnetic field sensitivity. This is attributed to an increasing rate of spin relaxation processes (similar to the $\mathrm{Ln}^{3+}$ effects reviewed above). The spin-rotational relaxation mechanism (going parallel to the $g$-factor anisotropy ${ }^{455}$ and showing heavy-atom enhancement) provides a good order-of-magnitude explanation for the effects observed.

Although several time-resolved MFEs with geminate radical pairs from singlet precursors have been observed 


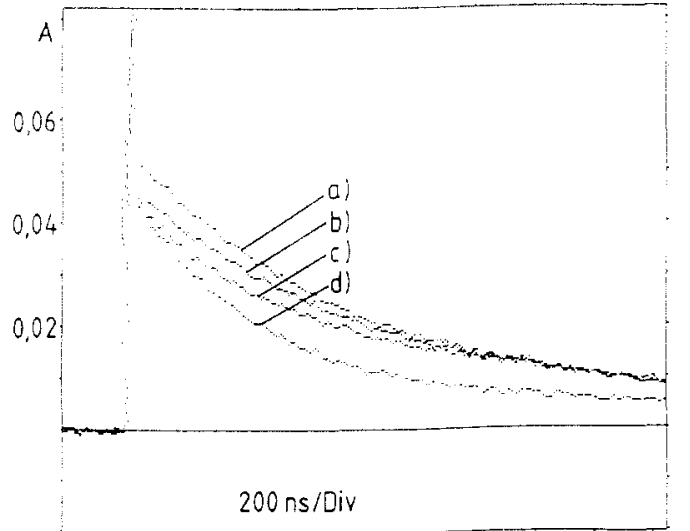

Figure 36. Kinetics of geminate radical pair decay from oxonine (6O) singlet quenching by $N, N$-tetramethyl- $p$-phenylenediamine in AOT/isooctane $/ \mathrm{H}_{2} \mathrm{O}$ microemulsion. Magnetic field strength (a) $0.0 \mathrm{mT}$, (b) $7.5 \mathrm{mT}$, (c) $50 \mathrm{mT}$, and (d) $1.0 \mathrm{~T}$ (from Baumann, Ulrich, and Steiner ${ }^{441}$ ).

in homogeneous solution (cf. Table 8) and in photosynthetic reaction centers (cf. section IV.F), there is so far only one case where MFEs have been observed with singlet radical pairs in micellar solution. Recently, a MFE on singlet-derived radical pairs has been found in AOT w/o microemulsions by Baumann et al. ${ }^{441}$ The radical pairs were produced in the electron-transfer reaction between TMPDA and oxonine singlet $(6 \mathrm{O})$. In a magnetic field these radical pairs recombine more efficiently than in zero field since suppression of singlet-triplet transitions will now tend to prolong the singlet-spin memory of the pair and keep its recombination rate higher than it would be for spin-equilibrized radical pairs since no recombination of triplet radical pairs is possible in this system. In Figure 36 typical radical pair decay curves are shown. They are characterized by a very fast initial recombination, within the duration of the laser pulse, and a second phase of recombination on the order of a few hundred nanoseconds. Both stages reflect geminate radical pair recombination. The initial, fast one, where the radical pair must be assumed to undergo frequent reencounters, shows a MFE governed by the hfc mechanism, which is indicated by a low $B_{1 / 2}$ value of about 6-8 $\mathrm{mT}$. The slower part of the recombination is interpreted by a trapping of both radicals in the surfactant/water interface, so that reencounters become much less frequent. Here the MFE is attributed to the relaxation mechanism because rather high magnetic fields are necessary to bring the relaxation rate below a threshold where it is slower than the rate of intramicellar reencounters of the radical pair.

In concluding this section, we emphasize once more that micelles or the nanodroplets of microemulsions are ideal systems to study spin correlation effects on the recombination of radical pairs over a fairly long time scale. Due to the rather slow escape of radicals from the microreactors, which is typically on the order of 1 $\mu \mathrm{s}$, the time scale for the observation of geminate recombination is extended by 2 or 3 orders of magnitude with respect to low-viscosity homogeneous solutions. It is due to this fact that very marked MFEs occur and that rather slow spin-flip processes, known from ESR to be responsible for electron spin relaxation in radicals, can greatly contribute to the multiplicity change of the radical pair during the intramicellar recombination period. Thus the MFD of spin relaxation processes is reflected in the recombination kinetics, which opens up a new access to the investigation of the former.

\section{E. Interface Phenomena}

Due to enhancement of cage effects, interfaces and surfaces should provide more favorable conditions for magnetic field effects than three-dimensional bulk phases. Theoretical treatments of pair diffusion in various dimensions ${ }^{456,457}$ have shown that geminate reencounters occurring with a probability of less than 1 in three dimensions have a probability of 1 in systems with two translational degrees of freedom. In spite of such favorable conditions, examples of magnetic field effects in two-dimensional systems are much less numerous than for three-dimensional ones. This may be due to experimental difficulties, since surfaces often do not have well-reproducible properties and there is also a lack of convenient instrumental methods for in situ chemical analysis.

From the latter point of view chemical processes at electrodes would be ideal to study. However, apart from certain photoprocesses at semiconductor surfaces, which will be mentioned below, no electrochemical magnetic field effects have been reported that are true examples of magnetic-field-dependent reaction kinetics. A number of reports have been published on MFEs in electrolysis and electrode kinetics (cf. Watanabe et al. ${ }^{458}$ and references given therein), but usually these effects can be explained in terms of the magnetohydrodynamic force inducing a flow of the solution enhancing or impeding the rate of diffusional mass transfer.

\section{Chemical Product Yields}

Turro and co-workers ${ }^{131,459}$ have studied cage effects and MFEs on the photodecomposition of several dibenzyl ketone derivatives adsorbed on porous glass, silica gel, and reversed-phase silica gel. The cage effects observed are similar to the micellar supercage effects, and significant MFEs have been observed, too. Thus the yield of 1,2-diphenylpropane, the cage product in the photolysis of $\alpha$-methyldibenzyl ketone (34a) decreases by $61 \%$ in a magnetic field of $0.3 \mathrm{~T}$ when the material is adsorbed on porous glass. ${ }^{131}$ The corresponding photoreaction of $p$-methyldibenzyl ketone (33a) was studied on silica of variable pore size. ${ }^{459} \mathrm{~A}$ correlation of cage effects and MFEs with pore size was found. MFEs ensued, however, only with a low degree of surface coverage. In order to explain the different degrees of cage effects and MFEs at low and high surface coverage, a two-site model of adsorption of the ketone on silica has been suggested. As should be expected, surface reactions of this type are also very favorable for magnetic isotope separation ${ }^{148,460}$ (cf. section IV.H).

There has been one report of a MFE on photosensitized $\mathrm{H}_{2}$ evolution at a semiconductor surface. Kiwi ${ }^{153}$ investigated the efficiency of $\mathrm{H}_{2}$ production from water, photosensitized by Ru(bpy) ${ }_{3}{ }^{2+}$ with EDTA as a sacrificial electron donor. The heterogeneous catalyst for $\mathrm{H}_{2}$ evolution was $\mathrm{RuO}_{2}$-doped $\mathrm{TiO}_{2}$, partially covered with Pt. The rate of $\mathrm{H}_{2}$ evolution in this system is decreased by $55 \%$ in a magnetic field of $0.4 \mathrm{~T}$. A MFE 
of the same size appears when methylviologen $\left(\mathrm{MV}^{2+}\right.$ (60)) is added to the system as an electron relay, al-

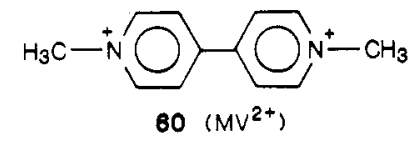

though under these conditions $\mathrm{H}_{2}$ evolution is about 40 times more efficient. A MFE is not observed with direct UV irradiation of the semiconductor suspension in the absence of the sensitizer system or on the yield of $\mathrm{MV}^{\circ+}$ radicals in the homogeneous $\mathrm{Ru}(\mathrm{bpy})_{3}{ }_{3}{ }^{2+} / \mathrm{MV}^{2+} / \mathrm{EDTA}$ system. A reinvestigation of the homogeneous system in our laboratory, however, has furnished a MFE of $-10 \%$ at $1 \mathrm{~T}$ on the yield of $\mathrm{MV}^{\circ+}{ }^{481}$ Recently, the effect was also investigated by Ferraudi and Argüello ${ }^{774}$ with high magnetic fields up to $5 \mathrm{~T}$, at which the yield of $\mathrm{MV}^{\circ+}$ was reported to be decreased by about $27 \%$.

\section{Photoluminescence and Photoconductivity}

MFEs on photocurrents in tetracene films deposited on anthracene crystals or silica plates were reported very early by Frankevich and co-workers. ${ }^{229}$ These phenomena, which have been reviewed by Sokolik and Frankevich, ${ }^{12}$ are characterized by an increase of the photoconductivity in a magnetic field. The explanation provided by Frankevich ${ }^{284}$ is given in terms of a kind of $\Delta g$ mechanism operating on a loosely bound charge pair (Wannier exciton) where singlet-triplet transitions are assumed to be induced by different $g$ factors of hole and electron. In fact, it seems to have been the first case where the $\Delta g$ mechanism was invoked. The same kind of mechanism is also thought to apply to the MFE on exciplex fluorescence observed from thin tetracene films on anthracene. ${ }^{461}$ The fluorescence intensity is reduced by a few percent, which is the saturating MFE reached at rather low fields of a few millitesla. In view of the small $B_{1 / 2}$ value $(1.6 \mathrm{mT})$ it appears very questionable, however, if a $\Delta g$ mechanism can be a reasonable explanation.

Also in Frankevich's group, the rate of formation of photooxidation products, when tetracene ${ }^{462}$ or rubrene $e^{463}$ films were illuminated in an oxygen-containing atmosphere, has been found to be increased by a magnetic field. The photooxidation rate was determined indirectly from the intensity of a photocurrent, which is enhanced by the oxidation products (presumably aromatic endoperoxides). The MFE has been tentatively explained in terms of the Merrifield (TT) pair mechanism for the singlet channel (formation of ${ }^{1} \mathrm{O}_{2}$ and ${ }^{1} \mathrm{MO}_{2}$ ) in the interaction of a triplet exciton with ${ }^{3} \mathrm{O}_{2}$. Since the ZFS of oxygen is rather large, at $B<0.2 \mathrm{~T}$ the Merrifield mechanism would predict an increase of the rate of singlet product formation with increasing magnetic field. Geacintov and Swenberg ${ }^{367}$ have analyzed the theoretical problem of MFEs on triplet-aromatic/triplet-oxygen pairs, concentrating especially on the energy splittings between quintet-, triplet-, and singlet-pair states, which depend on the extent of charge-transfer interaction between the aromatic triplet and oxygen molecule. Since they failed to see a MFE on phosphorescence quenching of coronene, chrysene, and dibenzoanthrecene adsorbed on polystyrene fluff, they concluded that the triplet-singlet splitting in such encounter pairs should exceed $30 \mathrm{~cm}^{-1}$.
When certain dyes are adsorbed on organic crystals a photosensitized hole injection into the molecular crystal may occur, causing a sensitized hole current or sensitized delayed fluorescence. ${ }^{464-466}$ The latter process involves electron-hole recombination with formation of triplet states, which on triplet-triplet annihilation lead to delayed fluorescence. After the primary electron transfer from the organic crystal to the adsorbed dye, the charge pair originating with singlet spin correlation (since $S_{1}$ is the photoactive excited state of the dye) may separate, whereby the mobile species is the positive hole, migrating by a hopping mechanism over the molecules of the crystal. Since it is attracted by its image charge in the solution, the motion of the hole remains restricted to the crystal surface for a fairly long time. ${ }^{176}$ During this stage, there will be spin evolution due to hyperfine interactions, predominantly influencing the fixed electron on the adsorbed dye molecule. On reencounter of the charge pair the spins may be triplet aligned, suitable for recombination to produce a triplet exciton. This situation is quite analogous to what has been described above for homogeneous solutions where the triplet recombination efficiency of singlet radical pairs can be influenced by magnetic fields. ${ }^{157,302}$

Merrifield and co-workers ${ }^{465,466}$ found that the delayed fluorescence of anthracene, sensitized by rhodamine, xanthene, and cyanine dyes is decreased by about $50 \%$ at fields higher than $20 \mathrm{mT}$. The half-field value of this effect was very small $(1-2.5 \mathrm{mT})$ and was related to the hyperfine coupling mechanism. In passing, it is of interest to note that, historically, this MFE on delayed fluorescence, sensitized by hole injection, was observed prior to the corresponding case in homogeneous solutions. Also, Merrifield and co-workers, when suggesting the hyperfine mechanism for the charge carrier pair in their first paper, ${ }^{465}$ were not aware of the obvious parallels to the radical pair mechanism that had been established some years before in connection with the CIDNP phenomenon. Actually, in this paper, they even argued against a possible importance of such a mechanism in solutions.

Hole injection into anthracene crystals by photoexcited tetramethylrhodamine has been studied by Michel-Beyerle and co-workers. ${ }^{175}$ They demonstrated that the MFE on sensitized hole current was complementary to that of delayed fluorescence. They also studied magnetic isotope effects in the anthracene crystal ( $\mathrm{H} / \mathrm{D}$ exchange) and in the dye $\left({ }^{14} \mathrm{~N} /{ }^{15} \mathrm{~N}\right.$ exchange). Only ${ }^{15} \mathrm{~N}$-substitution in the dye modified the MFE, yielding a lower $B_{1 / 2}$ value. $H / D$ exchange in anthracene was inefficient. This is explained by the fact that due to its hopping motion between anthracene molecules the electron hole only experiences randomized hyperfine coupling, which is much less efficient than a constant hyperfine coupling (cf. also section V).

This kind of investigation was further extended by Willig and co-workers, ${ }^{176}$ who also studied the dependence of the effects on applied voltage (cf. Figure 37). The higher the electric field, the faster is the separation of the hole from the electron, residing on the dye radical, and the MFE on the hole current decreases. Furthermore, using various methods of preparation of the anthracene crystals, shallow traps for the injected holes have been generated at the surface. These traps cause a characteristic change in the electric field de- 


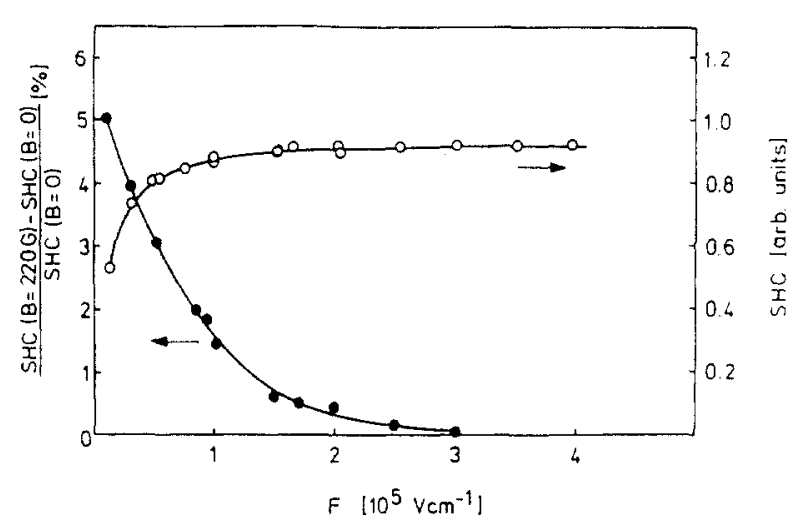

Figure 37. Rhodamine B sensitized hole current (SHC) and relative MFE at $220 \mathrm{G}$ in an anthracene single crystal as a function of electric field $\mathbf{F}$. Reprinted from ref 769 with kind permission of F. Willig; copyright 1988 Butterworths.

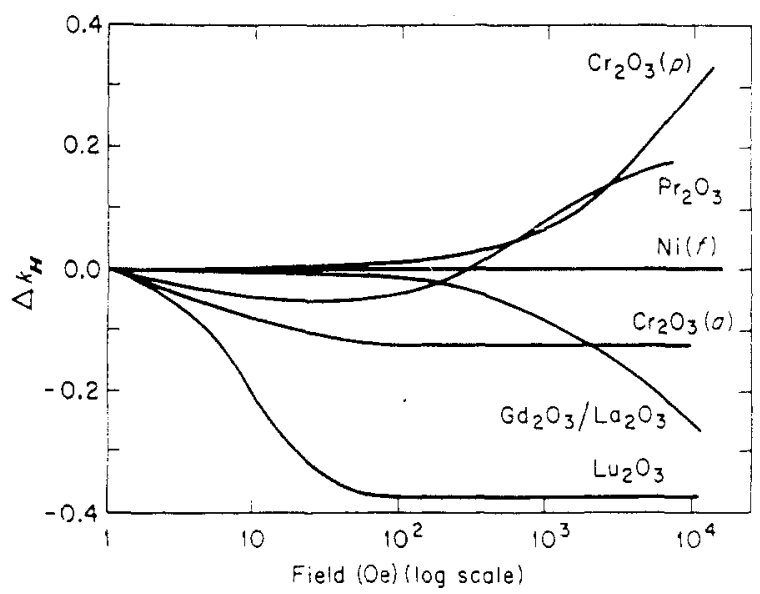

Figure 38. Relative change of catalytic $o / p-\mathrm{H}_{2}$ conversion rate $\left(\Delta k_{\mathrm{H}}\right)$ as a function of magnetic field strength for various magnetic materials: (a) antiferromagnetic, (f) ferromagnetic, (p) paramagnetic, and intrinsically diamagnetic substances. Reprinted with permission from ref 26; copyright 1985 Academic Press.

pendence of the MFE on the hole current. ${ }^{467}$ An appropriate theoretical model has been developed accounting for the observed effects (cf. section V).

\section{Magnetocatalytic Para to Ortho Hydrogen Conversion}

Nondissociative interconversion of para and ortho hydrogen has been known for a long time to be catalyzed by paramagnetic collisions (e.g., with $\mathrm{O}_{2}, \mathrm{NO}$, and $\mathrm{NO}_{2}$ in the gas phase 468 and in the liquid phase with transition-metal ions, ${ }^{469-473}$ trivalent lanthanon ions, ${ }^{469}$ and organometallic compounds ${ }^{472}$ ).

As has been shown by Wigner, ${ }^{82}$ this process can be explained by the inhomogeneous magnetic field of the paramagnetic center, which exerts different influences on the two proton spins of the $\mathrm{H}_{2}$ molecule during a collision. So far, however, no external MFEs have been reported to modify the reaction rate of this type of catalytic process in homogeneous systems. On the other hand, MFEs on the catalytic activity (magnetocatalytic effect) ${ }^{480}$ have been found for several paramagnetic and also some diamagnetic solids. A review of this work has been given by Selwood, ${ }^{26}$ and here we will only briefly consider some characteristic aspects.

The catalytic activity exhibited by magnetic oxides such as lanthana, lutetia, and yttria may be influenced by rather small magnetic fields ${ }^{26}$ (cf. Figure 38 ). Although these materials are inherently diamagnetic, it is thought that their catalytic activity results from paramagnetic centers on the surface which might be produced in the activation reaction wherein the materials are heated in a hydrogen atmosphere. The paramagnetic rare-earth sesquioxides have been extensively studied, ${ }^{26,153,155,474-476}$ either self-supported or lanthana-supported. In many of these systems the negative magnetocatalytic effect as described for lanthana has been observed in low fields up to $4 \mathrm{mT}$. In high fields, however, this effect is reversed and may reach positive MFEs up to $200 \%$ in fields of $1.8 \mathrm{~T}$ (cf. the example of $\mathrm{Pr}_{2} \mathrm{O}_{3}$ in Figure 38). Whereas with these paramagnetic oxides the absolute rate constants of $\mathrm{p} / \mathrm{o}$ hydrogen conversion follow a proportionality to the square of the effective magnetic moment, 26,477 as predicted by Wig. ner's theory, ${ }^{82}$ a consistent and quantitative theoretical explanation of the magnetocatalytic effect is not so far available (cf. section V).

The antiferromagnetic oxides $\alpha-\mathrm{Cr}_{2} \mathrm{O}_{3}, \mathrm{CoO}$, and $\mathrm{MnO}$ have been investigated with respect to their magnetocatalytic properties below and above their Neel temperatures. ${ }^{26,478}$ The catalytic activities showed maxima at the Neel temperatures $\left(T_{\mathrm{N}}\right)$, and the magnetocatalytic effect was negative in the paramagnetic region $\left(T>T_{\mathrm{N}}\right)$ and positive in the antiferromagnetic region $\left(T<T_{\mathrm{N}}\right.$ ) (cf. Figure 38 for the behavior of $\alpha$ $\mathrm{Cr}_{2} \mathrm{O}_{3}$, which shows the most abrupt changes at the Neel temperature).

Only few of the ferromagnetic solids are suitable for studies of the nondissociative $\mathrm{p} / \mathrm{o}$ hydrogen conversion, because the dissociative route often dominates. ${ }^{26}$ For $\mathrm{CrO}_{2}, \mathrm{EuO}$, and $\mathrm{Ni}$ nondissociative $\mathrm{p} / \mathrm{o}$ hydrogen conversion activity can be studied in certain temperature ranges. ${ }^{26,479}$ It has been found that below the Curie temperature there is no magnetocatalytic effect, whereas in the paramagnetic region these materials show a strong, positive external MFE. Typical MFD curves for the various magnetic types of the materials used are shown in Figure 38. Current theories to explain the magnetocatalytic effects on nondissociative $\mathrm{p} / \mathrm{o}$ hydrogen conversion will be reviewed in section $\mathrm{V}$.

\section{F. Blological Systems}

\section{General Situation}

In the past many magnetobiological or magnetophy. siological effects have been found and are described in a number of books. ${ }^{58,516-521}$ The origin of most of these effects is, however, not of molecular nature or not yet precisely known. Thus, e.g., the well-documented phenomenon of a magnetic sense of certain higher animals, particularly of birds, still awaits conclusive mechanistic explanation, though interesting hypothetical models have been suggested on the basis of the radical pair mechanism. ${ }^{514,522,523}$

There are, however, specific molecular biological systems for which MFEs have been observed in isolated, well-defined preparations, among which photosynthetic reaction centers are the most prominent. The MFE studies of these have by now reached a high standard and will be reviewed in section 2. Other magneticfield-dependent reaction systems of biological interest involve cytochrome-catalyzed oxidations and binding 
of $\mathrm{CO}$ to hemoglobin.

Molin and co-workers ${ }^{297}$ have reported that the rate of $\mathrm{O}_{2}$ evolution in $\mathrm{H}_{2} \mathrm{O}_{2}$ decomposition, catalyzed by the heme enzyme catalase, is increased by about $20 \%$ in a magnetic field of $0.8 \mathrm{~T}$. Marked MFEs have been also observed in the hydroxylation of organic substrates by cytochrome P-450, another heme enzyme. The effects are thought to be due to the $\Delta g$ mechanism increasing intersystem crossing in intermediate $\mathrm{Fe}^{3+} \mathrm{O}_{2}{ }^{*-}$ radical pairs. Here $\Delta g$ has the extremely high value of 2 , which is $100-1000$ times larger than in pairs of typical organic free radicals.

A different mechanism is responsible for the MFD found for the rate of $\mathrm{CO}$ rebinding to human $\beta$-hemoglobin after photolysis of the adduct below $20 \mathrm{~K}$. Rather strong fields (up to $10 \mathrm{~T}$ ) have been used to induce a weak optical linear dichroism, which is due to anisotropically enhanced recombination of the molecular complex in a magnetic field. ${ }^{524}$ The MFE is due to the fact that $\mathrm{CO}$ binding goes along with a high-spin $(S=2)$ to low-spin $(S=0)$ conversion of the complexed $\mathrm{Fe}^{\mathrm{II}}$ ion. The magnetic field causes changes in the effective SOC by recoupling the quintet zero-field substates $^{521,526}$ (cf. section V.B).

\section{Magnetic Field Effects with Photosynthetic Reaction Centers}

Photosynthetic primary processes occur in special membrane-fixed reaction centers in the chloroplasts of green plants, in algae, or in several kinds of bacteria. Typically, the primary process is denoted as

$$
\mathrm{P} * \mathrm{IX} \stackrel{2.8 \mathrm{ps}}{\longrightarrow} \mathrm{P}^{\cdot+} \mathrm{I}^{*-} \mathrm{X} \stackrel{\leq 200 \mathrm{ps}}{\longrightarrow} \mathrm{P} \cdot+\mathrm{IX} \cdot-
$$

Here $\mathrm{P}$ denotes the chlorophyll special pair, which in its first excited singlet states donates an electron to the "primary" acceptor X (a menaquinone-iron complex) with one detectable intermediate electron acceptor I (a bacteriopheophytin in the case of bacteria) only. ${ }^{527}$ Most investigations concentrate on the purple bacteria Rhodopseudomonas spheroides, mainly from the carotenoidless R-26 strain, and Rhodospirillum rubrum. Recently, it has been possible to crystallize the protein complex of reaction centers of Rhodopseudomonas viridis, a species closely related to Rhodopseudomonas spheroides. It has been possible to determine the crystal structure and obtain detailed information on the spatial configuration of the various pigments involved in the primary electron-transfer chain. ${ }^{528,529}$ In native photosystems electron transfer according to eq 64 is essentially irreversible. If, however, the electron acceptor $\mathrm{X}$ is either reduced or removed, the primary radical pair $\mathrm{P}^{++} \mathrm{I}^{*-}$ may undergo electron back-transfer in various ways represented in Figure 39 . Whereas the singlet ground state $\mathrm{P}$ can be regenerated directly, a multiplicity change in the radical pair $\mathrm{P}^{\mathrm{F}}$ is necessary to allow for (reversible) formation of the triplet state $\mathrm{P}^{\mathrm{R}}$ of the special pair, which is energetically somewhat below the radical pair state. From the singlet pair, electron back-transfer to regenerate the excited singlet state has to be considered, too.

In view of the general interest in the molecular mechanism of photosynthesis, great effort has been applied to utilize MFEs on triplet formation, groundstate repopulation, and delayed fluorescence in photo-

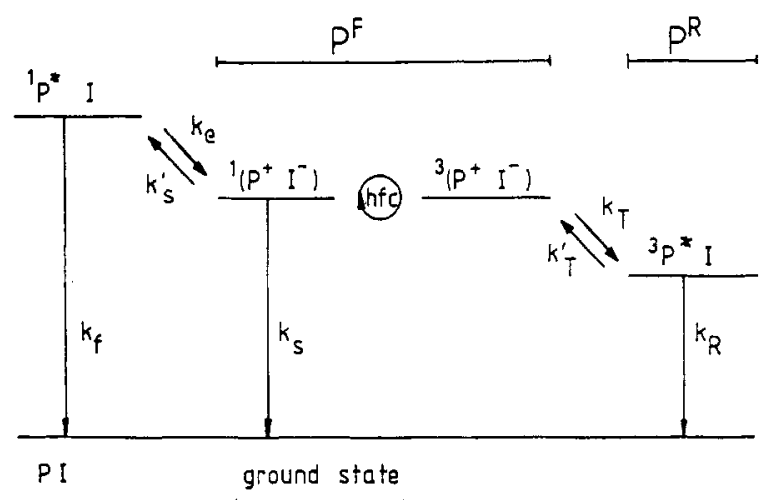

Figure 39. Reaction scheme of primary processes on prereduced or quinone-depleted bacterial photosynthetic reaction centers. $P$, special pair of bacteriochlorophylls; I, "intermediate" electron-accepting bacteriopheophytin (adapted from Werner, Schulten, and Weller ${ }^{532}$ ).

synthetic reaction centers. Furthermore, problems arising in connection with the interpretation of these results have instigated fundamental theoretical work which has largely contributed to the general understanding of magnetic field effects in chemical kinetics (cf. section V).

Reviews on MFEs in photosynthetic systems have been written by Hoff ${ }^{37,57}$ and by Boxer et al. ${ }^{45}$ Furthermore, these aspects have been treated in other reviews by $\mathrm{Hoff}^{44,51,530,531}$ on magnetic resonance investigations in photosynthesis, where also a useful introduction to the molecular photosynthetic machinery and the primary processes may be found, together with a number of experimental details and references on the preparation of suitable samples.

In this section we shall confine ourselves to tabulating the relevant experimental work (cf. Table 14) and giving a short outline of the main aspects under investigation. For more details of theoretical aspects, cf. section V.

Most investigations in the field deal with bacterial photosynthetic units, either in isolated reaction centers or in other preparations under reducing conditions or with the quinone complexes removed. MFEs were first detected by Blankenship et al. ${ }^{482}$ and by Hoff et al. ${ }^{483}$ on the yield of reaction center triplet. The triplet yield decreases in a magnetic field. The magnitude of the effects and the half-field values are critically dependent on the type of preparation of the system. Nevertheless the general results bear strong evidence that the effect is due to the hyperfine coupling mechanism in the radical pair. These results have prompted theoretical investigations ${ }^{532-534}$ studying the influence of the rate parameters $k_{\mathrm{S}}$ and $k_{\mathrm{T}}$ and the exchange interaction $J$ on the MFD.

In order to understand the relatively large $B_{1 / 2}$ value in some preparations, which considerably exceeds the value of the typical hyperfine coupling strength in the radical pair $\mathrm{P}^{\mathrm{F}}$, Werner et al. ${ }^{532}$ included the effect of the electron back-transfer (rate constant $k_{\mathrm{S}}^{\prime}$ ) leading to repopulation of the excited singlet state. In fact, such a reaction should give rise to a MFE on the delayed fluorescence, which actually has been observed. $485-487,493,496,498$ The effects on the total fluorescence yield are rather small since most of the fluorescence is prompt fluorescence from antenna bacteriochlorophylls. The effects on the delayed component ${ }^{500}$ are, however, of the same magnitude as on the yield of 
TABLE 14. Magnetic Field Effect with Photosynthetic Reaction Centers

\begin{tabular}{|c|c|c|c|}
\hline system $^{a}$ & $\operatorname{method}^{b}$ & observations ${ }^{c}$ & ref \\
\hline Rps. sph., reduced $R C s$ & LFP & $\begin{array}{l}R\left(\left[\mathrm{P}^{\mathrm{R}}\right]\right), R_{\mathrm{B}}=-50 \% \text { (at } R T, \text { smaller at lower temp), } B_{1 / 2} \leq 40 \mathrm{mT} \text {, } \\
\quad B_{\mathrm{s}} \approx 0.1 \mathrm{~T}\end{array}$ & $\begin{array}{l}1977 \\
\text { Blankenship et al. }{ }^{482}\end{array}$ \\
\hline$R p s . s p h .$, various preps & LFP & $\begin{array}{l}R\left(\left[\mathrm{P}^{\mathrm{R}}\right]\right),-40 \% \leq R_{\mathrm{m}} \leq-10 \%, 4 \leq B_{1 / 2} \leq 50 \mathrm{mT}, \text { depending on } \\
\quad \text { preparation }\end{array}$ & Hoff et al. ${ }^{483}$ \\
\hline Rps. sph., whole cells, reduced & $\mathrm{F}$ & $T=1.5 \mathrm{~K}: R_{\mathrm{S}}\left(I_{\mathrm{F}}\right)=1.1 \%, B_{\mathrm{S}}=30 \mathrm{mT}$, triplet mechanism & $\begin{array}{l}1978 \\
\text { Gorter de Vries } \\
\quad \text { and Hoff } \\
\quad 484\end{array}$ \\
\hline Rps. sph. R-26, reduced RCs & $\mathrm{F}$ & $R_{\mathrm{S}}\left(I_{\mathrm{F}}\right)=+1.2 \%, B_{1 / 2}=12 \mathrm{mT}$ & $\begin{array}{l}\text { Voznyak et al. }{ }^{487} \\
1979\end{array}$ \\
\hline $\begin{array}{l}\text { Rps. sph., Rsp. r., whole cells } \\
\text { and chromophores, Chlorella }\end{array}$ & $\mathrm{F}$ & $\begin{array}{l}R\left(I_{\mathrm{F}}\right), R_{\mathrm{S}} \approx+2 \%(295 \mathrm{~K}),+0.3 \%(175 \mathrm{~K}), \text { similar effects with } \\
\quad \text { photosystem II }\end{array}$ & Rademaker et al. ${ }^{485}$ \\
\hline
\end{tabular}
vulgaris, spinach chloroplasts, all reduced

Rps. sph. R-26, reduced RCs

Chromatium minutissimum, whole cells, reduced

Rps. sph. R-26, reduced RCs

Rsp. rubr., whole cells, also deuteriated, var red. potential Chlorella vulgaris, reduced spinach chloroplasts, reduced

Rps. sph., reduced RCs

pea chloroplasts, P-700 enriched, reduced

Rps. sph. R-26, RCs, Q depleted or reduced

Rps. sph., Rsp. r., Chromatium vinosum (chromophores red.)

Rps. sph, whole cells, reduced green leaves, in vivo

spinach chloroplasts, PS-I particles, reduced

Rps. sph. R-26, Rsp. r. (also deuteriated), various red. potl, RCs, Q or Fe depleted

Rps.sph. R-26, RCs, Q depleted

Rps. sph. R-26, RCs, Q depleted

in media of various viscosities

Rps. sph., wild type

Rps. sph. R-26, RCs, Q depleted

TP-LFP $R\left(\left[\mathrm{P}^{\mathrm{R}}\right]\right)$, var delay time, $R_{\mathrm{S}} \approx-70 \%, B_{1 / 2}(10.5 \mathrm{~ns})=7.5 \mathrm{mT}$, $B_{1 / 2}(16.5 \mathrm{~ns})=5.5 \mathrm{mT}$

F $\quad R_{\mathrm{S}}\left(I_{\mathrm{F}}\right)$ depends on preirradiation time (photosystem II effect)

A $\quad 150 \mathrm{~K}: R\left(\left[\mathrm{P}^{\mathrm{R}}\right]\right)=-22 \%(0.15 \mathrm{~T}),-12 \%(1.4 \mathrm{~T}), \Delta g$ effect

TP.LFP $R\left(\left[\mathrm{P}^{R}\right]\right) \approx-R\left(\left[\mathrm{P}^{\mathrm{F}}\right]\right)$, MFE obsd at var wavelengths

LFP $\quad R$ ([carotenoid triplet], $0.6 \mathrm{~T})=-10 \%$ (BC excited), $-45 \%$ (car. excited), two mechanisms, no $D$ effect

F $\quad R\left(I_{\mathrm{F}}\right)$ temp dep, $R(40 \mathrm{mT}, 80 \mathrm{~K}) \approx+3 \%$, no saturation

$\mathrm{F}, \operatorname{trF} \quad R\left(I_{\mathrm{DF}}\right)=+10 \%\left(0.2 \mathrm{~T}, R T, \tau_{\mathrm{DF}}=0.7 \mu \mathrm{s}\right),+40-50 \%(0.2 \mathrm{~T}, 77 \mathrm{~K}$, $\left.\tau_{\mathrm{DF}}=0.15 \mu \mathrm{s}\right)$, photosystem II effects

F $\quad R_{\mathrm{S}}\left(I_{\mathrm{F}}\right)=+5.5 \%(60 \mathrm{mT}), B_{1 / 2}=7.5 \mathrm{mT}(R T), R_{\mathrm{S}}\left(I_{\mathrm{F}}, T\right) \max$ at $230 \mathrm{~K}:+8 \%$

F $\quad R_{\mathrm{S}}\left(I_{\mathrm{F}}\right) \leq 2.5 \%, B_{1 / 2} \approx 30 \mathrm{mT}$, photosystem I effect

A $\quad R(\Delta[\mathrm{P}])=-44 \%(0.12 \mathrm{~T}), B_{1 / 2}=8 \mathrm{mT}(\mathrm{Q}$ depleted $), B_{1 / 2}=26$ $\mathrm{mT}$ ( $\mathrm{Q}$ reduced)

LFP $\quad R\left(\left[\mathrm{P}^{R}\right]\right), \mathrm{RYDMR}$ spectra

$\operatorname{tr} F \quad R\left(\tau_{F}\right) \approx+3$ to $7 \%$, weak $T$ dependence

F $\quad$ at $5 \mathrm{~K}: R\left(I_{\mathrm{F}}\right)>0$, depends on intensity of excitation

$\mathrm{F}\left(B_{0} \sim\right) \quad R\left(I_{\mathrm{F}}\right)=+0.15 \%, B_{1 / 2}=1.6 \mathrm{mT}$, after preillumination $R\left(\mathrm{I}_{\mathrm{F}}\right)=$ $+0.016 \%, B_{1 / 2}=20 \mathrm{mT}$

$\mathrm{F}, \operatorname{trF} \quad R\left(I_{\mathrm{F}}\right)=+1.5 \%, R\left(I_{\mathrm{DF}}, \tau=100 \mathrm{~ns}\right)=+20 \%$ temp and wavelength dep

$\mathrm{F}, \operatorname{trF}, \quad$ temp dep of $R\left(\left[\mathrm{P}^{\mathrm{R}}\right]\right), R\left(\tau\left(\mathrm{P}^{\mathrm{R}}\right)\right), R\left(I_{\mathrm{F}}\right), R\left(\tau\left(\mathrm{P}^{\mathrm{F}}\right)\right)$, no $\mathrm{H} / \mathrm{D}$ effect LFP

TP-LFP $R\left(\left[\mathrm{P}^{\mathrm{R}}\right]\right), B_{1 / 2}=8,6,4.1$, and $3.3 \mathrm{mT}$ at delay time of $3,5,7$, and $15 \mathrm{~ns}$

LFP $\quad R\left(\left[\mathrm{P}^{\mathrm{R}}\right]\right), B \leq 0.1 \mathrm{~T}, R(0.1 \mathrm{~T})=-50 \%, B_{1 / 2} \approx 4.2 \mathrm{mT}$, $0 \leq B \leq 5 \mathrm{~T}, R(5 \mathrm{~T})=+10 \%$

$R\left(\left[\mathrm{P}^{\mathrm{R}}\right]\right), 0 \leq B \leq 5 \mathrm{~T}$, magnetic anisotropy

LFP $\quad R\left(\left[\mathrm{P}^{\mathrm{R}}\right]\right), \mathrm{RYDMR}$ spectra

LFP $\quad$ RYDMR spectra: $\Delta R\left(5 \mathrm{ns:}\left[\mathrm{P}^{\mathrm{F}}\right]\right)<0, \Delta R\left(200 \mathrm{~ns}:\left[\mathrm{P}^{\mathrm{R}}\right]\right)>0$ $R\left(\tau\left(\mathrm{P}^{\mathrm{F}}\right)\right) \approx+33 \%, B_{1 / 2} \approx 5 \mathrm{mT}, \mathrm{MW}$ resonance effect on $\left.\tau\left(\mathrm{P}^{\mathrm{F}}\right)\right)$ $(1 \mathrm{~kW}: \Delta \tau<0 ; 7 \mathrm{~kW}: \Delta \tau>0)$

$\tau\left(\mathrm{P}^{\mathrm{F}}\right)(B): 13 \mathrm{~ns}(B=0 \mathrm{~T}), 17 \mathrm{~ns}(B=0.1 \mathrm{~T}), 9 \mathrm{~ns}(B=5 \mathrm{~T})$

Rps.sph. R-26, RCs, Q depleted

LFP

$R\left(\left[\mathrm{P}^{\mathrm{R}}\right]\right)$ parallel to $R\left(\tau\left(\mathrm{P}^{\mathrm{R}}\right)\right), 0<B<5 \mathrm{~T}$

T-dep MFE on $\left.\tau\left(\mathrm{P}^{\mathrm{R}}\right)\right)$

$\mathrm{A}\left(B_{0} \sim\right) \quad$ MODS technique for $\mathrm{T}-\mathrm{S}_{0}$ difference spectra

A, MW T-dep low-field, low-power RYDMR

Rps. sph., wild type, and

Rps.sph. R-26, RCs, Q depleted

Rps. sph. R-26, RCs, reduced

$\mathrm{A}\left(B_{0} \sim\right) \quad$ MODS: $\triangle \mathrm{OD}(\mathrm{T}-\mathrm{S})$ spectra, $T: 24-290 \mathrm{~K}$

$R\left(\left[\mathrm{P}^{\mathrm{R}}\right]\right)$, RYDMR spectra

Rps. sph. R-26, RCs, Q depleted

TP-LFP $R\left(\left[\mathrm{P}^{\mathrm{R}}\right], \tau=40 \mathrm{~ns}\right):$ MFD at $90-295 \mathrm{~K}, B_{1 / 2}$ is not temp dep, rate of ${ }^{3} \mathrm{P}^{\mathrm{F}} \rightarrow{ }^{3} \mathrm{P}^{\mathrm{R}}$ activationless
Michel-Beyerle et al. ${ }^{158}$

Klevanik et al. ${ }^{486}$

1980

Chidsey et al. 488

Michel-Beyerle et al. ${ }^{489}$

Rademaker et al. 490

Rademaker et al.491

Sonneveld et al. ${ }^{492}$

Voznyak et al. ${ }^{493}$

Voznyak et al. ${ }^{494}$

1981

Norris et al. ${ }^{495}$

Bowman et al. ${ }^{122}$ van Bochove et al. ${ }^{496}$

Proskuryakov et al. ${ }^{497}$ Tribel et al. ${ }^{498}$

Sonneveld et al..$^{498}$

1982

Schenk et al. ${ }^{500}$

Ogrodnik et al. ${ }^{501}$

Roelofs et al. ${ }^{502}$

Boxer et al. ${ }^{503}$

Boxer et al. 504,505

Norris et al. ${ }^{506}$

1983

Wasielewski et al. ${ }^{507}$

Wasielewski et al. ${ }^{608}$

1984

Chidsey et al. ${ }^{509}$

1985

Boxer $^{510}$

Chidsey et al..$^{511}$

Hoff et al. ${ }^{97}$

Moehl et al. ${ }^{107}$

Norris et al. ${ }^{512}$

1986

Lous and Hoff, 513

Hoff and Lous ${ }^{51}$

1987

Ogrodnik et al. ${ }^{\text {115 }}$

a Photosynthetic bacteria: Rps. sph., Rhodopseudomonas spheroides; R-26, carotinoid less mutant of Rps. sph.; Rsp. r., Rhodospirillum rubrum; RC, isolated reaction center protein complex; $Q$, quinone constituent of RC. ${ }^{b} \mathrm{~A}$, absorption spectroscopy under steady-state illumination; $B_{0} \sim$, modulation of $B_{0}$ field; F, stationary fluorescence; trF, time-resolved fluorescence; LFP, laser flash photolysis; TP-LFP, two-pulse laser flash photolysis. ${ }^{c} I_{F}, I_{\mathrm{DF}}$, intensity of fluorescence, delayed fluorescence; MFD, magnetic field dependence, unless otherwise stated of case 1 type (specified by $R_{\mathrm{S}}, B_{\mathrm{S}}, B_{1 / 2} ;$ cf. Figure 6 ); MODS, magnetooptical difference spectroscopy; $\mathrm{P}^{\mathrm{R}}$, special pair triplet; $\mathrm{P}^{\mathrm{F}}$, radical pair $\mathrm{P}^{\bullet+} \mathrm{I}^{\bullet-} ; \Delta R$ change of $R$ due to resonant microwave (MW) irradiation; $R($ ) relative change of specified quantity; $\tau$, delay time of probing pulse; $\tau_{\mathrm{X}}, \tau(\mathrm{X})$ time constant of process $\mathrm{X}$, or lifetime of species X. 
$\mathrm{P}^{\mathrm{R}}$, i.e., up to several $10 \%$. Rademaker and Hoff ${ }^{535}$ have set up a general kinetic scheme relating the results of theoretical model calculations to the quantities actually observed under experimental conditions. This work demonstrates that, in principle, the hyperfine coupling mechanism can account for the effects observed.

Under conditions of continuous illumination a certain fraction of reaction centers will be in the $\mathrm{P}^{\mathrm{R}}$ state with a concomitant depletion of the ground state $P$. The magnetic-field-dependent rate of $\mathrm{P}^{\mathrm{R}}$ population will be reflected in a corresponding MFD of the $\mathrm{P}^{\mathrm{R}}$ steady-state concentration. Such effects have been utilized by Chidsey et al. ${ }^{488}$ and by Norris et al. ${ }^{495}$ to investigate the MFD of $\mathrm{P}^{\mathrm{R}}$ formation yield. Furthermore, the MFE on steady-state concentrations of $\mathrm{P}^{\mathrm{R}}$ and $\mathrm{P}$ can be exploited for sensitive monitoring of the $\triangle O D$ spectrum of these species. The method has been systematically developed as a novel spectroscopic technique (MODS (magnetooptical difference spectroscopy)) by Hoff and co-workers. ${ }^{97,513,514}$

Michel-Beyerle et al. ${ }^{158,501}$ have investigated the MFE on the yield of $\mathrm{P}^{\mathrm{R}}$ detected at variable delay time after the phorolyzing laser pulse. Their results exhibit a lifetime-broadening effect whereby the $B_{1 / 2}$ values increase as the delay time is decreased in the nanosecond region. The observed MFD of $\mathrm{P}^{\mathrm{R}}$ yield has been reproduced by a theoretical model employing two sites of the intermediate acceptor radical $\mathrm{I}^{\circ-}$. This model had been suggested by Haberkorn et al. ${ }^{534}$ in order to explain an obvious discrepancy between the electronic matrix element of the forward electron transfer (whereby $\mathrm{P}^{\mathrm{F}}$ is formed) and the upper limit of a few gauss for the exchange interaction in $\mathrm{P}^{\mathrm{F}}$ derived from the MFEs. It was suggested that the radical electron on $\mathrm{I}^{-}$is exchanged between two sites with different separation from the special pair radical $\mathrm{P}^{\bullet+}$. This view has been supported by recent X-ray diffraction results, showing a bacteriochlorophyll between the special pair and the bacteriopheophytin molecule which is adjacent to the menaquinone-Fe complex. However, recent time-resolved experiments with an 80-fs time resolution $^{527}$ afforded no evidence of the involvement of the accessory bacteriochlorophyll as a transient electron acceptor. Furthermore, in a recent paper by Ogrodnik et al. ${ }^{515}$ it was derived from the weak temperature dependence of $k_{\mathrm{T}}$ that the electronic matrix element responsible for forward electron transfer is about 25 times larger than for back-transfer (whereby $\mathrm{P}^{\mathrm{R}}$ is formed). Since Haberkorn et al., ${ }^{534}$ when suggesting their two-site radical pair model, had started out from the assumption of equal matrix elements for electron transfer in both directions, their interpretation is inconsistent with the recent findings. The asymmetry of the coupling elements of forward and backward electron transfer is attributed to a so-called superexchange mechanism, where the accessory bacteriochlorophyll functions as a virtual electron relay.

Since $\Delta g$ in $\mathrm{P}^{\mathrm{F}}$ is of the order of $0.001,{ }^{488}$ the MFE is of case 1 type up to fields of $0.1 \mathrm{~T}$. A dominating contribution of the $\Delta g$ mechanism was, however, established by Boxer et al., ${ }^{488,503}$ who applied magnetic fields up to $5 \mathrm{~T}$. Thus, whereas the triplet yield is significantly decreased in low fields, in the high-field region the yield of $\mathrm{P}^{\mathrm{R}}$ may be even increased above the zero-field value.
Boxer and co-workers ${ }^{504,505}$ have also found a significant magnetic anisotropy of the effect when using bacterial reaction centers suspended in highly viscous media. The magnetic anisotropy is borne out in the comparison of measurements with the magnetic field axes parallel or perpendicular to the electric field vector of the exciting light. The anisotropy of electron spinspin interaction in the radical pair or a $\Delta g$ anisotropy was considered as a possible reason for this effect. Recent theoretical results obtained by Ogrodnik et al. ${ }^{536}$ for the spin-dipolar interaction between the various radical species based on the crystal structure data of Deisenhofer et al. ${ }^{528}$ indicated that this type of interaction is probably too weak to be extracted from magnetic-field-dependent reaction fields (MARY) or RYDMR experiments.

Recombinations to the ground state $\mathrm{P}$ (rate constant $k_{\mathrm{S}}$ ) and to the triplet state $\mathrm{P}^{\mathrm{R}}$ (rate constant $k_{\mathrm{T}}$ ) represent the main decay channels of the radical pair $\mathrm{P}^{\mathrm{F}}$. From the sign of the MFE on the triplet yield it follows consistently that $k_{\mathrm{T}}$ must be larger than $k_{\mathrm{S}}$. This relation is also borne out in the results of $\mathrm{P}^{\mathrm{F}}$-lifetime measurements. ${ }^{508,511}$ Chidsey et al. ${ }^{511}$ found that the lifetime of $\mathrm{P}^{\mathrm{F}}$ increases at low magnetic fields, reaching a maximum at about $0.1 \mathrm{~T}$, and decreasing again to a value that at $B=5 \mathrm{~T}$ is even smaller than for zero field. Since, according to the radical pair mechanism, the singlet-triplet conversion rate is slowed down at low fields (hyperfine mechanism) and increased in high fields ( $\Delta g$ mechanism), it follows from the MFD of the $\mathrm{P}^{\mathrm{F}}$ lifetime that the triplet radical pair decay to $\mathrm{P}^{\mathrm{R}}$ must be faster than the singlet radical pair decay to $P$. A MFE that parallels the effect on the yield of $P^{R}$ has been also found on the lifetime of $\mathrm{P}^{\mathrm{R}}$. 510,511 The MFE on $\mathrm{P}^{\mathrm{R}}$ lifetime disappears at low temperature. These results indicate that at room temperature $\mathrm{P}^{\mathrm{R}}$ decays to a considerable amount through thermally activated formation of $\mathrm{PF}^{\mathrm{F}}$. From the temperature dependence of the effect a reaction energy of $0.12 \mathrm{eV}$ has been obtained.

There have been attempts to observe $H / D$ magnetic isotope effects on the yield of triplets from charge recombination in reaction center triplets. ${ }^{490,500}$ Surprisingly, no deuterium effect on the yield of $\mathrm{P}^{\mathrm{R}}$ occurred.

In comparison with bacterial photosynthetic reaction centers there are rather few investigations available dealing with MFE studies in photosystems of plants. Fluorescence investigations of preparations showing preferential delayed fluorescence of either photosystem $\mathrm{I}^{494,499}$ or photosystem $\mathrm{II}^{491,492}$ have revealed, however, that similar MFEs as observed in bacterial reaction centers occur in photosystems of plants, too.

In the reaction sequences of Figure 39 or eq 64 the radical pair state $\mathrm{PF}^{\mathrm{F}}$ is normally too short-lived to be directly detectable by ESR spectroscopy. However, in the case of prereduced primary acceptor $\mathrm{X}$ spin polarization developing during the lifetime of $\mathrm{P}^{\mathrm{F}}$ may be transferred to the paramagnetic species ${ }^{2} \mathrm{X}^{\bullet-}$ or ${ }^{3} \mathrm{P}\left(=\mathrm{P}^{\mathrm{R}}\right)$ and may be used to derive information on magnetic interactions during the lifetime of $\mathrm{P}^{\mathrm{F}}$. Furthermore, in the case of nonprereduced $\mathrm{X}$, the secondary pair $\mathrm{P}^{\cdot+}$. IX -- may be conveniently studied by ESR since its lifetime is of the order of milliseconds. ${ }^{537}$ For a survey of the literature on electron spin polarization $\mathrm{cf}$. the reviews by Hoff. ${ }^{34,44,51,57,530,531}$ Although the radical pair 
$\mathrm{P}^{\mathrm{F}}$ is too short-lived for direct ESR measurement, it is amenable to magnetic resonance detection if the RYDMR technique is used. Several studies of this type ${ }^{107,122,506-508}$ are included in Table 14. For further comments on these, cf. sections IV.G and V.E.

The effects and observations described so far in this section are all attributed to the radical pair mechanism in the primary radical pair $\mathrm{P}^{\mathrm{F}}$. It should be mentioned that the MFD of fluorescence from suspensions of reduced whole cells of Rhodopseudomonas spheroides at $1.4 \mathrm{~K} \mathrm{have}$ been related to the triplet mechanism. ${ }^{484}$ It may be assumed that the triplet sublevels of $\mathrm{P}^{\mathrm{R}}$ are kinetically isolated at $1.4 \mathrm{~K}$. Then mixing of the sublevels by an external magnetic field will increase the overall triplet decay rate with a concominant decreases of the stationary $\mathrm{P}^{\mathrm{R}}$ concentration.

Reaction centers in the $\mathrm{P}^{\mathrm{R}}$ state do not act as energy traps of electronic excitation in the antenna system. Thus a change of $\mathrm{P}^{\mathrm{R}}$ population is borne out by a change of antenna pigment fluorescence. The MFE on fluorescence observed at $5 \mathrm{~K}$ and very high intensity of exciting light, however, does not seem to be explicable in terms of this mechanism. ${ }^{497}$

Finally, it shall be mentioned that a MFE on carotinoid triplet formation in whole cells of Rhodopseudomonas rubrum has been attributed to the singlet fission mechanism. ${ }^{490}$

\section{G. Reaction-Yield-Detected Magnetlc Resonance}

The RYDMR method is a logical extension of the original ODMR technique. In fact, the terminological borderline between them is not always very sharp, since in RYDMR as in ODMR optical methods of detection are usually applied.

In optically detected magnetic resonance (ODMR) of electronically excited states usually photoluminescence intensities are probed under microwave irradiation while a static magnetic field is tuned through the resonance. This technique was introduced into atomic Zeeman spectroscopy in the vapor phase by Brossel and Bitter. ${ }^{105}$ Further applications of the method of atomic spectroscopy has been reviewed by Series. ${ }^{558}$ The ODMR method was soon extended to investigate magnetic electronically excited states in inorganic ${ }^{100}$ and organic solids. ${ }^{101-104}$ Due to the anisotropy of intrinsic magnetic interactions in ligand field states and molecular triplet states, ODMR became feasible even in zero field. For surveys of these subjects, cf. the reviews by Cavenett, ${ }^{117}$ El-Sayed, ${ }^{561}$ and Hausser and Wolf, ${ }^{562}$ and Kinoshita et al. ${ }^{563}$

Under conditions where repopulation of magnetic sublevels of an electronically excited state by microwave irradiation can be observed as ODMR, the resonant microwave effect (absorption or enhanced emission) will also change the lifetime of the magnetic substates, which may cause a change of chemical product yields if specific chemical reactions take place in the excited states. Thus the occurrence of triplet-state ODMR indicates that chemical reaction yields can be changed by microwave irradiation according to the triplet mechanism.

An example of such a behavior is the two-photon photodecomposition of pyrimidine observed by Leung and El-Sayed ${ }^{279}$ at $1.6 \mathrm{~K}$ (cf. also section IV.B), which

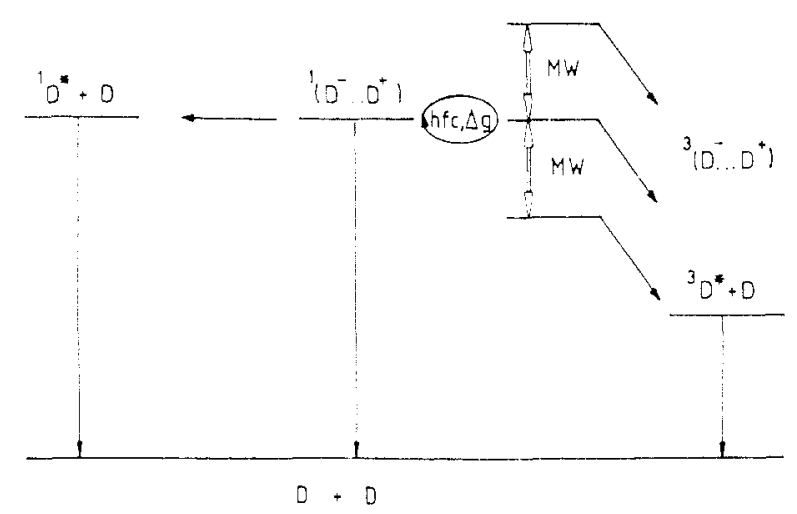

Figure 40. Basic kinetic scheme accounting for RYDMR effects in radical pair recombination reactions.

has been modified by microwave resonance in zero field.

The observability of ODMR effects requires that a detectable change of quasi-stationary sublevel populations can be achieved by the microwaves. This means that thermal equilibrium among the magnetic sublevels should not be established during the decay of the excited state. Such conditions are normally maintained at very low temperatures of several kelvins only, which is a typical condition for triplet-state ODMR, monitored through phosphorescence. Recently, Norris et al., ${ }^{512}$ however, reported a RYDMR signal detected at room temperature by a resonant change of transient absorption intensity in photosynthetic reaction centers (cf. Table 15), which they tentatively attributed to a triplet-state magnetic resonance signal.

A rather peculiar effect of an irregular series of sharp resonances on the growth of yeast cultures under the influence of microwaves near $42 \mathrm{GHz}$ in zero magnetic field has been reported by Grundler et al. ${ }^{564,565}$ The resonances correspond to approximately $\pm 10 \%$ modulation of the growth rate with bandwidths of only 8 $\mathrm{MHz}$. It has been suggested that they might correspond to zero-field triplet sublevel transitions in a triplet species supposed to be essential in yeast metabolism. ${ }^{566}$ However, its identity has not been determined, and it seems hard to understand why the triplet sublevels should not be in thermal equilibrium at room temperature.

In the literature the term RYDMR is normally used to characterize microwave effects upon processes (luminescence emission or other decay, including chemical reactions) involving one of the spin-selective pair mechanisms outlined in section II. The possibility that such mechanisms should also respond to resonant microwave transitions in a static magnetic field was first discussed by Kubarev and Pshenichov. ${ }^{567}$

The principle may be explained by using the radical pair spin-level reaction scheme in Figure 40 . Here specific exit channels exist for radical pairs with singlet and triplet spin alignment, respectively. The spin levels are coupled, though, by local magnetic interactions such as hyperfine coupling in radicals or dipolar electronic spin-spin coupling if triplets in T-T or T-D pairs are concerned. In the case of a radical pair two triplet levels are decoupled from the singlet level as an external magnetic field is applied. This decoupling causes a change in the yield of triplet and singlet recombination products. If resonant microwave transitions within the triplet manifold are induced, the decoupling effect due to the static magnetic field is partially removed and a 
TABLE 15. Experiments with Reaction-Yield-Detected Magnetic Resonance (RYDMR)

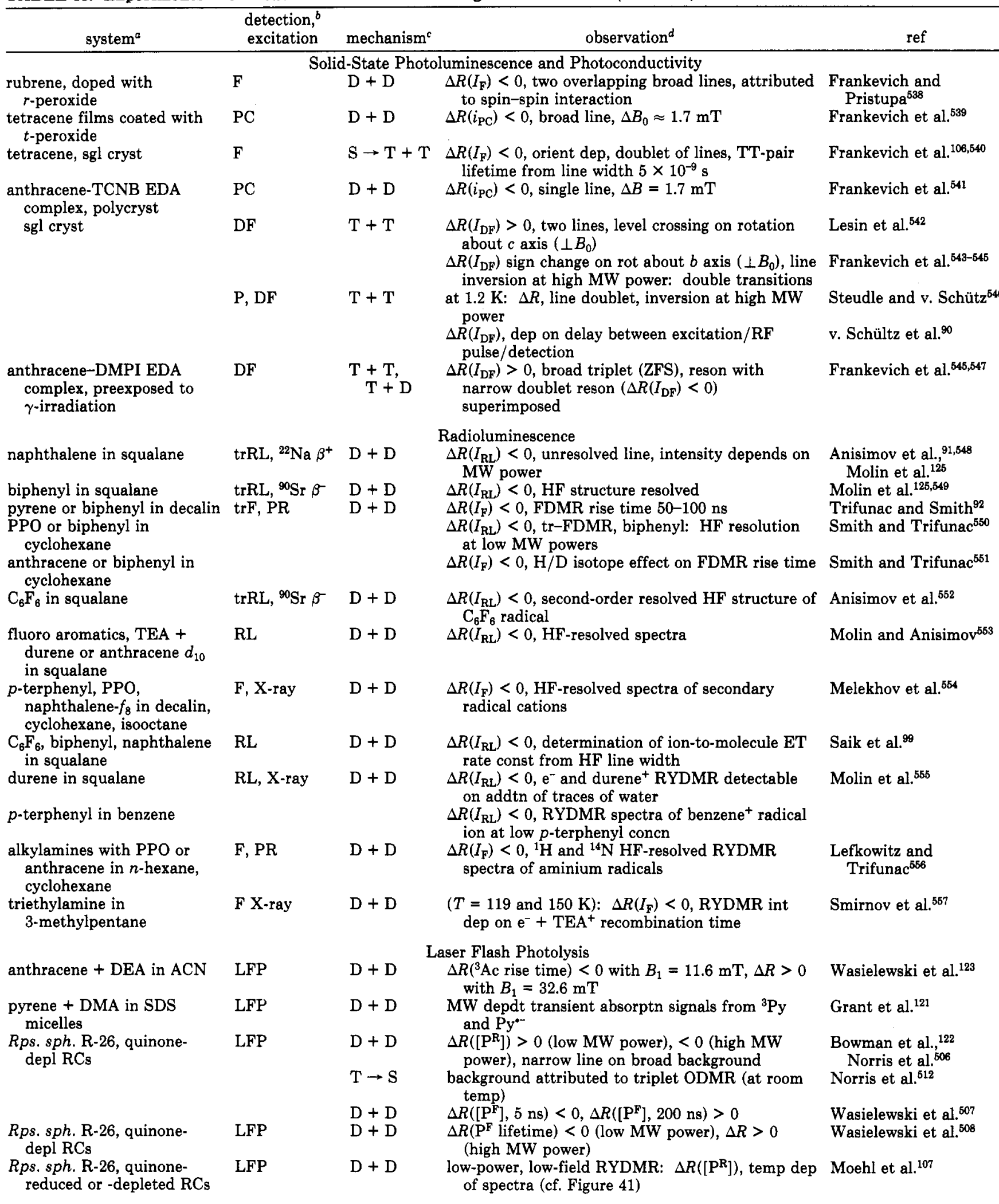

a DEA, $N, N$-diethylaniline; DMA, $N, N$-dimethylaniline; DMPI, pyromellitic $N, N$ '-dimethyldiimide; EDA, electron donor-acceptor; PPO, 2,5-diphenyloxazole; $R p s$. sph. R-26 RC, Rhodopseudomonas spheroides R-26 strain reaction centers; SDS, sodium dodecyl sulfate; TCNB, 1,2,4,5-tetracyanobenzene; TEA, triethylamine. ${ }^{b} \mathrm{DF}$, delayed fluorescence; F, fluorescence; LFP, laser flash photolysis; $\mathrm{P}$, phosphorescence; $\mathrm{PC}$, photoconductivity; $\mathrm{PR}$, pulse radiolysis; $\mathrm{RL}$, radioluminescence; tr, time-resolved. ' $\mathrm{AC}$, anthracene; $\mathrm{CT}$, charge transfer; $\Delta R(X)$, intensity of RYDMR line, monitored through quantity $X$; FDMR, fluorescence-detected magnetic resonance; HF, hyperfine; $I$, luminescence intensity; $i_{\mathrm{PC}}$, photocurrent; $\mathrm{MW}$, microwave; $\mathrm{P}^{\mathrm{F}}$, primary radical pair in photosynthetic reaction center; $\mathrm{P}^{\mathrm{R}}$, triplet of photosynthetic reaction center; ZFS, zero-field splitting.

RYDMR effect ensues that is opposite to the static MFE (MARY effect).

On the other hand, if by virtue of a g-factor difference of the radicals the static magnetic field couples $\mathrm{S}$ and $\mathrm{T}_{0}$ radical pair states, resonant microwave transitions to or from $\mathrm{T}_{0}$ will support the kinetic flow from $\mathrm{S}$ to the 
$T$ manifold and vice versa. In this case the RYDMR effect will have the same sign as the static MFE.

A different feature of microwave effects is shown at high microwave power (high $B_{1}$ ). If the microwave-induced coupling of the triplet substates $\left(g_{\mu_{\mathrm{B}}} B_{1}\right)$ exceeds the intrinsic $S-T_{0}$ coupling of the pair (given by the hfc constants or by the term $\left.\Delta g \mu_{B} \beta_{0}\right)$, the $S \leftrightarrow T_{0}$ transitions will be suppressed and the RYDMR effect exhibits a sign inversion as $B_{1}$ is increased.

The principle outlined with the example of the radical pair mechanism is likewise applicable to mechanisms involving other pairs such as T-T and T-D pairs. As compared with conventional ESR spectroscopy, RYDMR spectroscopy exhibits several unique features. Its great versatility with respect to the physical quantities detected (fluorescence, delayed fluorescence, photoconductivity, transient absorption, etc.) is combined with a high sensitivity and a high time resolution with respect to the species probed in the RYDMR spectra. These species, i.e., correlated pairs of paramagnetic particles, are usually too short-lived to be detected by conventional ESE techniques. Due to the short lifetimes of the species observed, the spectral line shape is often determined by kinetic factors, rather than by energetic fine structure.

The first observation of a pair mechanism type RYDMR effect was reported in 1976 by Frankevich and Pristupa, ${ }^{538}$ who found a resonant microwave effect upon the fluorescence of rubrene doped with its own peroxide. An overlapping doublet of broad lines was observed, which has been attributed to electron-donor electron-acceptor radical pairs exhibiting dipolar spinspin interaction. A variety of RYDMR spectra have been reported since, many of which are reviewed in an article by Frankevich and Kubarev. ${ }^{41}$ In Table 15 we have listed the relevant experimental work discussed in that review together with further recent experimental work.

So far RYDMR investigations have been applied to a great variety of systems, i.e., organic crystals, inorganic semiconductors, scintillator solutions, and micellar solutions, but also in biological systems such as bacterial reaction centers and even living systems like yeast cells (vide supra). Among the detection methods luminescence measurements figure prominently, certainly due to their superior sensitivity. Actually stationary concentrations as low as 20 spins in a sample are detectable. ${ }^{91,553}$ In fact, such luminescence investigations might be classified by the older term ODMR if it were not to emphasize that the underlying mechanism in the cases considered here is related to spin-selective reaction channels of pairs of paramagnetic particles that are otherwise free to diffuse, whereas ODMR is normally used in connection with magnetic transitions in molecular triplet states.

With respect to the mechanism causing the RYDMR effects, the radical pair or D-D pair mechanism is clearly the most frequent one encountered. It has been found in all types of systems mentioned above. So far, examples based on T-T pair and T-D pair reactions or on singlet fission seem to be restricted to molecular crystals.

Due to lifetime broadening the spectral resolution of RYDMR is lower than in conventional ESR with long-lived paramagnetic species. Hyperfine resolution has been observed, though, in a number of cases ( $\mathrm{cf}$.

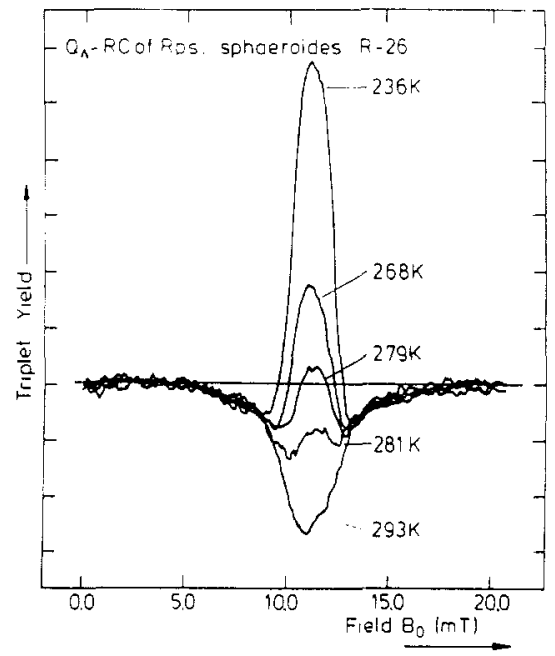

Figure 41. Low-power, low-field RYDMR spectra of quinonedepleted reaction centers of Rps. spheroides R26 at various temperatures. Reprinted from ref 107 with kind permission of A. Hoff; Elsevier Science Publishers B.V.

Table 15), especially with nuclei of strong hyperfine coupling (e.g., fluoro aromatics). Quantitative calculation of RYDMR line shapes requires a theoretical consideration of both spin Hamiltonian and kinetic processes. These aspects will be reviewed in section V.

From an analysis of RYDMR spectra, based on a suitable theoretical model, detailed information may be obtained concerning pair kinetics and magnetic interactions. Such approaches have been particularly useful in studies of photosynthetic reaction centers. In the latter systems signals were detected by time-resolved optical absorption spectroscopy combined with laser flash photolysis. With this method, resonance effects not only on reaction yields but also on lifetimes have been detected. ${ }^{123,508}$

In order to avoid line broadening due to high microwave power, Moehl et al. ${ }^{107}$ adopted a stationary-state absorption technique to measure RYDMR spectra at low microwave power (cf. also section III). Examples of RYDMR spectra in photosynthetic reaction centers of purple bacteria observed with this technique at different temperatures are depicted in Figure 41. Here it is shown that the high-temperature negative RYDMR line undergoes a line inversion in the center when the temperature is lowered, whereas the wings of the line remain unaffected. From the spectra an upper limit of $0.42 \times 10^{9} \mathrm{~s}^{-1}$ to the sum of decay rates of the primary photoinduced radical pair could be fixed which is significantly lower than that from previous work. For a more detailed discussion, cf. section V.

Another field closely related to the subject of this section, which we will only mention briefly, though, is spin-dependent charge carrier recombination in semiconductors such as silicon, ${ }^{179,568} \mathrm{Si} \mathrm{p}-\mathrm{n}$ junctions, ${ }^{177}$ hydrogenated amorphous silicon ${ }^{108,178,569,570}$ (reviewed by Street ${ }^{40}$, and semiconducting phosphors ${ }^{571,572,575}$ (reviewed by Cavenett ${ }^{117}$ ). The spin-pairing requirement for charge carrier recombination, which was first emphasized by Lepine, ${ }^{568}$ gives rise to static MFEs (cf. section IV.B) and resonant microwave effects, which have been observed by monitoring photoconductivity or photoluminescence.

A first explanation of these phenomena was based on the argument that, due to thermal spin polarization in 
an external magnetic field, encounters of charge carriers with parallel spins, which cannot recombine, will be a little more probable than encounters with antiparallel spins, which can lead to recombination. Resonant microwave absorption will decrease the bulk spin polarization and hence increase the recombination probability. This model, however, underestimated the observed effects by 1-2 orders of magnitude. ${ }^{178}$

That the situation in spin-dependent charge recombination is completely analogous to the radical pair mechanism with recombining $\mathrm{F}$ pairs in chemical kinetics was not recognized at first, and the motion of correlated spin pairs and the idea that an excess of triplet-spin correlated pairs is produced as a consequence of spin-dependent recombination was introduced as a mechanism of its own by Kaplan et al. ${ }^{573}$ who treated this situation within a semiclassical model. The connection with current theories of RYDMR in radical pairs was established later by Haberkorn and Dietz $^{574}$ (cf. section V).

In concluding this section we wish to note that not only resonant microwave but also radio-frequency transitions may be used to modify the reactivity of radical pairs. Such effects are detectable by a change of CIDNP intensities in the products formed from the radical pairs wherein the nuclear transitions occur. The method was first applied by Sagdeev et al. ${ }^{110}$. to photochemical reactions carried out directly in the probe of an NMR spectrometer. Analogous experiments with radical pairs from pulse radiolysis were performed by Trifunac and Evanochko. ${ }^{89}$ These workers used a further developed technique employing a flow system that allowed them to perform radical pair NMR in a separate magnetic field before the sample was transferred to the NMR magnet wherein the CIDNP signal was detected. CIDNP-detected magnetic resonance of radical pairs is a kind of ENDOR spectroscopy. Hyperfine coupling constants and kinetic parameters of radical pairs can be obtained from it.

Another most remarkable example of NMR-induced rate enhancement of radical reactions has been reported by Yurke et al. ${ }^{560}$ for the reaction of electron-spin-polarized hydrogen $(\mathrm{H} \downarrow)$ at low temperature. In a magnetic field the lowest two hyperfine levels " $a$ " and " $b$ " correspond to a pure triplet alignment of electron spin and nuclear spin (b) or an approximate $50 / 50 \mathrm{~S} / \mathrm{T}$ mixture (a). On the other hand, the electronic-nuclear spin wave function of ground-state molecular ortho/ para hydrogen does not contain contributions of atomic $\mathrm{b} \times \mathrm{b}$ products. Therefore only the collisions $\mathrm{H} \downarrow(\mathrm{a})-$ $\mathrm{H} \downarrow$ (a) or $\mathrm{H} \downarrow$ (a)-H $\downarrow$ (b) can lead to recombination, which means that $\mathrm{H} \downarrow$ (a) is preferentially depleted and a nuclear spin polarization (surplus of $H \downarrow(b)$ ) ensues, together with a slowing down of the recombination rate as the fraction of $\mathrm{H} \downarrow$ (b) grows. NMR transitions $H \downarrow$ (b) $\rightarrow \mathrm{H}_{\downarrow}$ (a) will then increase the recombination rate again. This effect has been observed at $0.23 \mathrm{~K}$ in a magnetic field of $8.3 \mathrm{~T}$.

\section{H. Magnetic Isotope Effects}

Whenever multiplicity changes due to hyperfine coupling receive kinetic relevance in a step of a reaction mechanism, magnetic isotope effects on overall reaction rates, quantum yields, or chemical yields are to be expected. So far all effects of this kind that have been reported are based on the radical pair mechanism. There are two principal cases in which MIEs can be observed:

(a) When magnetic (nonmagnetic) nuclei are substituted by nonmagnetic (magnetic) isotopes, changes in the behavior of chemical compounds may be found which are shown in different zero-field reaction yields and kinetics, as well as in the MFD of these quantities. Such comparative studies have been performed with $\mathrm{H} / \mathrm{D}$ substitutions in a number of cases (cf. Table 16) but also with some ${ }^{12} \mathrm{C}-{ }^{13} \mathrm{C}$ substitutions. ${ }^{146,405,406,424,442}$

(b) If compounds containing isotopic mixtures, e.g., of natural abundance (cf. Table 16), are subjected to reactions involving radical pair intermediates, the difference in hyperfine coupling of the corresponding isotopes will cause a different distribution of isotopes between cage and escape product channels, so that chemical isotope separation takes place, leading to magnetic isotope enrichment in either cage or escape product, whichever requires a radical pair multiplicity change in order to become more favorable.

The characteristics of MIEs have been explicated in some detail by Buchachenko. ${ }^{589,590}$ It must be emphasized that MIEs can be much larger than mass isotope effects. Lawler ${ }^{591}$ has formulated three criteria to distinguish between mas and magnetic isotope effects:

(i) Whereas mass isotope effects depend monotonically on the mass of the isotopes to be compared, such a relation does not exist for the MIE. A particular case where this has been put to test by Turro et al. ${ }^{148,460}$ is the $\mathrm{O}$ isotopes ${ }^{16} \mathrm{O},{ }^{17} \mathrm{O}$, and ${ }^{18} \mathrm{O}$, where only the middle one is magnetic.

(ii) MIEs are highest in zero magnetic field or at low field strength comparable to the hyperfine coupling constant of the isotopic atom under consideration (for experimental examples, cf. Turro et al. ${ }^{407,408}$ ).

(iii) MIEs change sign if the multiplicity of the radical pair precursor is changed between singlet and triplet.

These criteria follow from the radical pair mechanism in a straightforward manner.

As was noted by Buchachenko, ${ }^{50}$ one should be aware that MIEs could be well responsible for unusual isotopic distributions in geochemical and cosmic material, and the hypothesis of chemical fractionation should not be discarded on the basis of considering mass isotopic effects only. ${ }^{592}$ In fact, Haberkorn et al. ${ }^{582}$ have discussed the possibility that the radical pair mechanism might account for an unusual ${ }^{13} \mathrm{C}$ isotope enrichment in the $\mathrm{H}$-adjacent carbon position of cyanoacetylene detected by microwave spectroscopy in interstellar molecular clouds. ${ }^{581}$

Magnetic isotope separation or enrichment is of obvious interest in itself, but also as a mechanistic tool for probing the kinetics of radical pair mechanisms. ${ }^{53,54}$ As such, magnetic isotope enrichments have an advantage over MFE investigations in that they do not require comparison of experiments with zero-field reference experiments carried out under otherwise exactly identical conditions. Only the isotope distribution of some product (or the educt after stopping the reaction) has to be compared with that of the educt (before the reaction). According to Buchachenko, ${ }^{50}$ two basic schemes ((65) and (66)) for magnetic isotope separation may be distinguished.

In case of scheme 65 escape product (EP) and cage product (CP) formation are irreversible, and hence the 
TABLE 16. Magnetic Isotope Effects (MIE)

\begin{tabular}{|c|c|c|c|}
\hline chem system ${ }^{a}$ & chem reaction, expt ${ }^{b}$ & appearance of $\mathrm{MIE}^{c}$ & ref \\
\hline $\begin{array}{l}p \text {-terphenyl in benzene, } \\
\text { decalin }\end{array}$ & radiolysis & $\begin{array}{c}\text { Hydrogen: }{ }^{1} \mathrm{H} /{ }^{2} \mathrm{H} \\
\text { MFD of radioluminescence, } \mathrm{H} / \mathrm{D} \text { difference }\end{array}$ & Brocklehurst ${ }^{165,384}$ \\
\hline naphthalene in squalane & $\gamma$-radiolysis & $\begin{array}{l}\mathrm{MFD}\left(B_{1 / 2} \text { and } R_{\mathrm{S}}\right) \text { of radioluminescence, } \mathrm{H} / \mathrm{D} \\
\text { difference }\end{array}$ & Dixon et al. ${ }^{380}$ \\
\hline $\begin{array}{l}\text { pyrene }+ \text { arom donors or } \\
\text { acceptors in polar solv }\end{array}$ & photo-ET, DF & MFD of ${ }^{3} \mathrm{Py}$ yield, $\mathrm{H} / \mathrm{D}$ effect on $B_{1 / 2}$ & $\begin{array}{l}\text { Werner et al., }{ }^{303} \text { Nolting } \\
\text { et al., }{ }^{305} \text { Weller et al. }{ }^{306}\end{array}$ \\
\hline & $\begin{array}{l}\text { photo-ET A }\left(B_{0} \sim\right) \\
\text { photo-ET, TP-LFP }\end{array}$ & $\begin{array}{l}\text { MFD of Acc- yield, } H / D \text { effect on } B_{1 / 2} \\
\text { MFD of }{ }^{3} \mathrm{Ac} \text { yield, } \mathrm{H} / \mathrm{D} \text { effect on } B_{1 / 2}\end{array}$ & $\begin{array}{l}\text { Bube et al. } \\
\text { Krüger et al. }{ }^{118}\end{array}$ \\
\hline ketones $\mathbf{3 6 a}$ in mic soln & $\mathrm{CP}, \alpha$-cleavage & $\begin{array}{l}\text { H/D effect on quantum yield of photodecomposition } \\
\text { and its MFD }\end{array}$ & Turro and Mattay ${ }^{409}$ \\
\hline $\mathrm{BP}(41)$ in SOS mic soln & LFP, H-abstr & $\begin{array}{l}\text { MFE on radical escape from mic larger for BP- } d_{10} \\
\text { than for BP- } h_{10}\end{array}$ & Sakaguchi et al. ${ }^{424}$ \\
\hline DBK (33) in homog solv & $\mathrm{CP}, \alpha$-cleavage & $\begin{array}{l}\text { Carbon: }{ }^{12} \mathrm{C} /{ }^{13} \mathrm{C} \\
{ }^{13} \mathrm{C} \text { enrichment in recovered } \mathrm{DBK} \text {, low single-stage } \\
\text { sep factor }\end{array}$ & $\begin{array}{l}\text { Buchachenko et al., } \\
\text { Turro and Kräutler, }{ }^{402}, \\
\text { Turro et al. }{ }^{404}\end{array}$ \\
\hline DBK (33) in solv of var visc & & ${ }^{13} \mathrm{C}$ enrichment, increase with solv visc & $\begin{array}{l}\text { Tarasov and Buchachenko, }{ }^{576} \\
\text { Sterna et al. }{ }^{577} \text { Turro } \\
\text { et al., }{ }^{143,578} \text { Tarasov et al. }{ }^{579}\end{array}$ \\
\hline $\mathrm{DBK}(\mathbf{3 3})$ in mic soln & & $\begin{array}{l}{ }^{13} \mathrm{C} \text { enrichment, high single-stage sep factor, } \\
\text { comparison of }{ }^{13} \mathrm{C} \text { enrichment in recovered } \mathrm{DBK} \\
\text { and other cage product }\end{array}$ & $\begin{array}{l}\text { Turro and Kräutler, }{ }^{402} \text { Turro } \\
\text { et al., }{ }^{14,404,408,578} \text { Kräutler } \\
\text { and Turro, }{ }^{405} \text { Tarasov } \\
\text { and Buchachenko }{ }^{144,145}\end{array}$ \\
\hline & & $\begin{array}{l}\text { MFD of }{ }^{13} \mathrm{C} \text { enrichment } \\
\text { position dependence of }{ }^{13} \mathrm{C} \text { enrichment }\end{array}$ & $\begin{array}{l}\text { Turro et al. }{ }^{407} \\
\text { Turro et al., }{ }^{413} \text { Tarasov } \\
\text { and Buchachenko }{ }^{144}\end{array}$ \\
\hline & & $\begin{array}{l}{ }^{13} \mathrm{C} \text { enrichment, correlation with photochem } \\
\text { quantum yield }\end{array}$ & $\begin{array}{l}\text { Kräutler and Turro, }{ }^{405,406} \\
\text { Turro et al. }{ }^{146}\end{array}$ \\
\hline DBK, various ${ }^{12} \mathrm{C} /{ }^{13} \mathrm{C}$ mixt & $\begin{array}{l}\text { photoinit emulsion } \\
\text { polymerization }\end{array}$ & MIE and MFE on mol wt distribution of polymer & Turro et al. ${ }^{414}$ \\
\hline $\begin{array}{l}\text { DBK (33) on por silica } \\
\text { ketones } 34 \mathbf{b}\left(d_{6}, d_{8}\right), 34 \mathbf{e}\left(d_{2}\right)\end{array}$ & $\begin{array}{l}\mathrm{CP}, \alpha \text {-cleavage } \\
\mathrm{LFP}, \alpha \text {-cleavage }\end{array}$ & $\begin{array}{l}{ }^{33} \mathrm{C} \text { enrichment in cage prod, high separation factor } \\
\text { saturation of kinetic MIE at } B>10 \mathrm{mT}\end{array}$ & $\begin{array}{l}\text { Turro et al. } \\
\text { Turro et al. }{ }^{767}\end{array}$ \\
\hline ketones $36 \mathbf{a}, \mathbf{b}$ in mic soln & $\mathrm{CP}, \alpha$-cleavage & MIE on MFD of cage product quantum yield & $\begin{array}{l}\text { Turro and Mattay, } \\
\text { Turro et al. }{ }^{119}\end{array}$ \\
\hline $\begin{array}{l}\text { ketones } 36, \text { PAK in homog } \\
\text { and mic soln }\end{array}$ & $\mathrm{CP}, \alpha$-cleavage & high ${ }^{13} \mathrm{C}$ enrichment of cage prod only in mic soln & Turro et al. ${ }^{578}$ \\
\hline cyclododecanones & $\mathrm{CP}, \alpha$-cleavage & ${ }^{13} \mathrm{C}$ enrichment with biradical intermediate & Klimenko et al. ${ }^{580}$ \\
\hline $\mathrm{BP}\left({ }^{13} \mathrm{C}\right.$ enr $)$ in mic soln & $\begin{array}{l}\text { LFP, } \mathrm{H} \text { abstr from } \\
\text { surfactant }\end{array}$ & ${ }^{13} \mathrm{C}$ influence on MFD of radical decay kinetics & Sagakuchi et al. ${ }^{24}$ \\
\hline $\begin{array}{l}\left.\text { BP }\left({ }^{13} \mathrm{C} \text { enr }\right)+52 \text { (H donor }\right) \\
\text { in mic soln }\end{array}$ & LFP, $\mathrm{H}$ abstr & ${ }^{12 / 13} \mathrm{C}$ difference only small & $\begin{array}{l}\text { Scaiano and Abuin, } \\
\text { Scaiano et al. }\end{array}$ \\
\hline $\begin{array}{l}\text { dibenzoylperoxide (15) } \\
\text { cyanoacetylene }\end{array}$ & $\begin{array}{l}\mathrm{CP},{ }^{3} \text { sens decomp } \\
\text { thermal decomp } \\
\text { radioastronomy }\end{array}$ & $\begin{array}{l}{ }^{13} \mathrm{C} \text { enrichment in phenylbenzoate (cage prod) } \\
{ }^{\text {weak }}{ }^{13} \mathrm{C} \text { enrichment in escape prod benzene } \\
{ }^{13} \mathrm{C} \text { enrichment in interstellar clouds } \\
\text { hypothesis of radical pair mechanism }\end{array}$ & $\begin{array}{l}\text { Sagdeev et al.96 } \\
\text { Buchachenko } 0^{50} \\
\text { Churchwell et al. } .^{582} \\
\text { Haberkorn et al. }\end{array}$ \\
\hline $\begin{array}{l}\text { tetramethylrhodamine on } \\
\text { anthracene crystals }\end{array}$ & PC, DF, photo-ET & $\begin{array}{c}\text { Nitrogen: }{ }^{14} \mathrm{~N} /{ }^{15} \mathrm{~N} \\
{ }^{14,15} \mathrm{~N}: \text { different } B_{1 / 2} \text { of MFD }\end{array}$ & Bube et al., ${ }^{175}$ Bube $^{583}$ \\
\hline ethylbenzene (liq) $+\mathrm{O}_{2}$ (gas) & radical init oxidation & $\begin{array}{l}\text { Oxygen: }{ }^{16} \mathrm{O} /{ }^{17} \mathrm{O} /{ }^{18} \mathrm{O} \\
{ }^{17} \mathrm{O} \text { enrichment in recovered oxygen }\end{array}$ & $\begin{array}{l}\text { Belyakov et al., } \\
\text { Buchachenko et al. }{ }^{585}\end{array}$ \\
\hline polymeric olefins $+\mathrm{O}_{2}$ (gas) & $\begin{array}{l}\text { radical and radiat } \\
\text { init oxidation }\end{array}$ & ${ }^{17} \mathrm{O}$ enrichment in recovered oxygen & Buchachenko et al. ${ }^{585-588}$ \\
\hline $\begin{array}{l}\text { ketones } 33,36 \mathrm{a}, \mathrm{c}\left({ }^{17} \mathrm{O},{ }^{18} \mathrm{O}\right)^{d} \\
\text { in mic soln or on silica }\end{array}$ & $C P, \alpha$-cleavage & high ${ }^{17} \mathrm{O}$ enrichment of cage prod on silica & Turro et al. 148,460 \\
\hline endoperoxide $4\left({ }^{17} \mathrm{O},{ }^{18} \mathrm{O}\right)^{d}$ & thermolysis & ${ }^{17} \mathrm{O}$ enrichment in ${ }^{3} \mathrm{O}_{2}$ if ${ }^{1} \mathrm{O}_{2}$ is trapped & $\begin{array}{l}\text { Turro and Chow, } \\
\text { Turro et al. }{ }^{295}\end{array}$ \\
\hline $\begin{array}{l}\text { triethylgermane + benzyl } \\
\text { chloride } \\
\text { triethylgermane + aromatic } \\
\text { ketones }\end{array}$ & thermal reaction & $\begin{array}{l}\text { Germanium } \\
\text { MFD of cage prod yield indicates feasibility of }{ }^{73} \mathrm{Ge} \\
\text { MIE }\end{array}$ & $\begin{array}{l}\text { Leshina et al., }{ }^{291} \\
\quad \text { Taraban et al. } \\
\text { Hayashi et al. } .^{426}\end{array}$ \\
\hline
\end{tabular}

ketones

${ }^{a} \mathrm{ACN}$, acetonitrile; BP, benzophenone; DEA, $N, N$-diethylaniline; DMA, $N, N$-dimethylaniline; DBK, dibenzyl ketone; PAK, phenyl adamantyl ketone; SOS, sodium octyl sulfate. ${ }^{b} \mathrm{CP}$, continuous photolysis; DF, delayed fluorescence; ET, electron transfer; LFP, laser, flash photolysis; $\mathrm{A}\left(B_{0} \sim\right)$, magnetic-field-modulated steady-state absorption spectroscopy; PC, photocurrent; TP-LFP, two-pulse laser flash photolysis. 'Ac, anthracene; MFD, magnetic field dependence; MFE, magnetic field effect; MIE, magnetic isotope effect. ${ }^{d}$ Sample preenriched in specified isotopes.

relative isotope enrichment of $\mathrm{CP}$ and $\mathrm{EP}$ is independent of the degree of conversion of $\mathrm{A}$.
In case of scheme 66 radical pair formation from a starting material $\mathrm{A}$ is partially reversible (cage recom- 


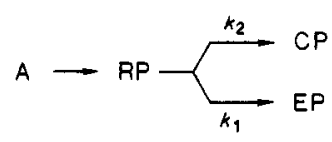

bination). Depending on the initial multiplicity of the radical pair, magnetic isotopes are entiched either in EP or in the recovered starting material A. After

$$
A \underset{k_{2}}{\rightleftharpoons} \operatorname{RP} \stackrel{k_{1}}{k_{3}} \mathrm{EP}
$$

complete conversion all isotopes are collected in the product EP with the same distribution as initially present in A. Thus, if the enrichment occurs in EP, it will decrease from a maximum value at low conversion to zero at complete conversion. If it is enriched in the starting material $\mathrm{A}$, however, the enrichment will continue to increase and will be at maximum if $\mathrm{A}$ is practically all consumed.

Numerous investigations pertaining to scheme 66 have been performed with dibenzyl ketone photolysis as a model reeaction (cf. Table 16). In this case the radical pair produced by $\alpha$-cleavage of the ketone is of triplet origin, and ${ }^{13} \mathrm{C}$-isotope enrichment is observed in the starting material, regenerated in the cage reaction. In micellar solutions there is another cage product (CP in scheme 66) ( $p$-tolyl benzyl ketone (TBK)) formed with an appreciable yield ${ }^{405,408}$ and also enriched in ${ }^{13} \mathrm{C}$. In this case the functional dependence of magnetic isotope enrichment on the degree of conversion is different for both A and EP. ${ }^{144,145}$

The following quantities are in use when dealing with magnetic isotope enrichments in chemical reactions quantitatively: ${ }^{50,54,143,590,594}$ (i) the magnetic isotope content $\delta$ of molecule A (A* denoting the magnetic isotope substituted molecule)

$$
\delta=\left[\mathrm{A}^{*}\right] /[\mathrm{A}]
$$

(ii) the magnetic isotope enrichment $S$ with respect to the starting situation

$$
S=\delta / \delta_{0}
$$

(iii) the conversion factor $f$ of starting material without magnetic isotope

$$
f=1-[\mathrm{A}] /[\mathrm{A}]_{0}
$$

(iv) the total conversion factor $f_{\exp }$

$$
f_{\exp }=1-\frac{[\mathrm{A}]+\left[\mathrm{A}^{*}\right]}{[\mathrm{A}]_{0}+\left[\mathrm{A}^{*}\right]_{0}}
$$

from which $f$ may be obtained according to

$$
f=f_{\exp } \frac{1+\delta_{0}}{1+\delta}
$$

and (v) the kinetic magnetic isotope effect $\gamma$

$$
\gamma=k^{*} / k
$$

(The rate constant $k$ in eq 72 has to be identified with the rate constant $k_{2}$ in schemes 65 and 66 , where it is sensitive to hyperfine coupling.)
TABLE 17. Nuclear Spins and Moments of Isotopes Investigated for Magnetic Isotope Effects ${ }^{a}$

\begin{tabular}{llcc}
\hline isotope & spin & $\mu_{\mathrm{N}}^{b}$ & nat abundance, \% \\
\hline${ }^{1} \mathrm{H}$ & $1 / 2$ & 2.79278 & 99.985 \\
${ }^{2} \mathrm{H}$ & 1 & 0.85742 & 0.015 \\
${ }^{12} \mathrm{C}$ & 0 & & 98.89 \\
${ }^{13} \mathrm{C}$ & $1 / 2$ & 0.7024 & 1.11 \\
${ }^{14} \mathrm{~N}$ & 1 & 0.4036 & 99.63 \\
${ }^{15} \mathrm{~N}$ & $1 / 2$ & -0.2831 & 0.37 \\
${ }^{16} \mathrm{O}$ & 0 & & 99.759 \\
${ }^{17} \mathrm{O}$ & $5 / 2$ & -1.8937 & 0.037 \\
${ }^{18} \mathrm{O}$ & 0 & & 0.2204 \\
${ }^{33} \mathrm{~S}$ & $3 / 2$ & 0.6433 & 0.76 \\
${ }^{73} \mathrm{Ge}^{c}$ & $9 / 2$ & -0.8792 & 7.76 \\
${ }^{115} \mathrm{Sn}$ & $1 / 2$ & -0.918 & 0.35 \\
${ }^{117} \mathrm{Sn}$ & $1 / 2$ & -1.000 & 7.61 \\
${ }^{119} \mathrm{Sn}{ }^{c}$ & $1 / 2$ & -1.046 & 8.58
\end{tabular}

a From Weast, R. C., Ed. CRC Handbook of Chemistry and Physics, 58th ed.; CRC Press: West Palm Beach, FL, 1978. ${ }^{b}$ Nuclear magnetic moment in multiples of nuclear magnetons. ${ }^{c}$ Rest of natural abundance is distributed over several nonmagnetic isotopes.

In order to describe isotopic enrichment in the starting material A for processes following scheme 66, one defines a single-stage separation factor $\alpha$

$$
\alpha \equiv \frac{\Phi_{\text {esc }}^{*}-\Phi_{\text {esc }}}{\Phi_{\text {esc }}}=\frac{1-\gamma}{k_{1} / k_{2}+\gamma}
$$

With this the following relation holds: ${ }^{50}$

$$
\log S=\alpha \log (1-f)
$$

from which, by measuring $S$ as a function of conversion $f$, the characteristic parameter $\alpha$ may be evaluated.

Characteristic data of various isotopes considered so far in experimental investigations on the MIE are collected in Table 17.

The first isotope enrichments using kinetic MIEs were reported by Buchachenko et al. ${ }^{95}$ and by Sagdeev et al. ${ }^{96} \mathrm{Up}$ to now, however, most experimental contributions to this field have been made by Turro and co-workers (cf. Table 16). The aspect of isotope enrichment by the MIE and its use as a mechanistic probe into radical pair reactions have been extensively reviewed by Buchachenko, ${ }^{19,50}$ by Turro and co-workers, ${ }^{36,39,42,48,53,54}$ and by Salikhov et al. ${ }^{52}$ Therefore we have confined ourselves to a compilation (Table 17) of the various chemical systems studied for MIEs. Only a few comments emphasizing some selected features of the work done so far will follow.

A number of experimental investigations devoted to the study of MFEs according to the radical pair mechanism have compared the influence of deuteriation on the magnetic field dependence (some examples are in Table 16, but cf. also section IV.C). Although there are marked influences of such substitutions, no $\mathrm{H} / \mathrm{D}$ isotope separation on the basis of the magnetic isotope effect has been reported. Continuous-photolysis experiments by Turro and Mattay ${ }^{409}$ with ketone 36a in micellar solution exhibited different cage production yields for $H$ and $D$, providing the possibility of separating these isotopes.

In fact, most work concerned with the magnetic isotope effect involves the ${ }^{13} \mathrm{C}$ isotope. These are obvious experiments when dealing with organic radicals and are reinforced by the fact that spin-orbit coupling effects inducing electron spin relaxation are not important in 
this case. An appreciable natural abundance of ${ }^{13} \mathrm{C}$ allows the use of natural material and easy detection of enrichment by NMR spectroscopy. The preferred model compound to study the ${ }^{13} \mathrm{C}$ magnetic isotope has been dibenzyl ketone (33), the reaction pathways for photolysis of which have been given above (cf. Figure 31). Remarkable progress in this field was made by the discovery of the micellar supercage effect, ${ }^{402}$ whereby enormous enhancements of the single-stage enrichment factor could be achieved, amounting up to $\alpha=1.47 .402,405$ In principle, efficient magnetic isotope enrichments should be also expected for reactions involving longchain biradical intermediates. With this intention Klimenko et al. ${ }^{580}$ investigated the ${ }^{13} \mathrm{C}$ enrichment in cyclododecanones upon reversible photolysis. The enrichment factors were, however, not extraordinarily high, which was attributed to a low reversibility of biradical formation in these systems.

The micellar supercage effect and its sensitivity to magnetic isotope effects have also been exploited for emulsion photopolymerization, where a significant ${ }^{13} \mathrm{C}$ isotope effect on molecular weight distribution has been found by Turro et al. ${ }^{414}$ Furthermore, enhancement of cage effects due to restricted diffusion on surfaces has also been demonstrated to be favorable for ${ }^{13} \mathrm{C}$ enrichment. ${ }^{460}$

Apart from $\alpha$-cleavage reactions of ketones, ${ }^{13} \mathrm{C}$ magnetic isotope effects have also been shown to occur in the photoreduction of benzophenone in micellar solutions. ${ }^{424,442,443}$ Triplet-sensitized decomposition of dibenzoyl peroxide was one of the first examples of ${ }^{13} \mathrm{C}$ enrichment. ${ }^{96}$ Thermal decomposition of this compound leads to singlet pairs, which may recombine in a spin-allowed process to yield only minor ${ }^{13} \mathrm{C}$ enrichment. 50

Another series of examples of magnetic isotope enrichments of a fairly light element have been reported for ${ }^{17} \mathrm{O}$. One type of reaction is thermolytic decomposition of endoperoxides, which has been investigated by Turro and co-workers. ${ }^{147,295}$ Thermolysis of compound 3 yields ${ }^{1} \mathrm{O}_{2}$ and ${ }^{3} \mathrm{O}_{2}$. As to be expected, the magnetic isotope ${ }^{17} \mathrm{O}$ is enriched in ${ }^{3} \mathrm{O}_{2}$, whereas the nonmagnetic isotopes ${ }^{16} \mathrm{O}$ and ${ }^{18} \mathrm{O}$ have a slight preponderance to be found in the singlet-oxygen trapping product. These experiments represent an example of a magnetic isotope effect with a trio of isotopes where the middle isotope is selectively enriched due to its magnetic properties, as anticipated by Lawler's first criterion (vide supra). The radical pair intermediate in the thermolysis of endoperoxides must be a diradical species, and it is remarkable that hyperfine coupling effects can establish themselves against the strong exchange interaction to be expected in these systems.

Enrichment of ${ }^{17} \mathrm{O}$ has been studied by Buchachenko and co-workers ${ }^{584-588}$ in some oxidation reactions with molecular oxygen. The substrates used were hydrocarbon polymers and ethylbenzene. Enrichment of ${ }^{17} \mathrm{O}$ is detected in the recovered oxygen in the gas phase. Magnetic isotope selection in these systems is believed to occur during encounters of free peroxy radicals (cf. scheme 75). F pairs are known to behave similarly to

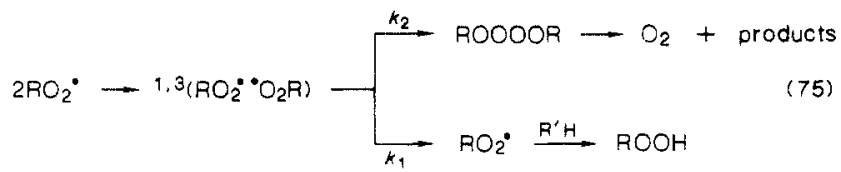

triplet pairs, and hyperfine coupling of terminal ${ }^{17} \mathrm{O}$ atoms will favor triplet-singlet transitions in these pairs so that tetraoxide formation takes place more efficiently. Consequently, molecular oxygen evolving from subsequent tetraoxide decomposition will be enriched in ${ }^{17} \mathrm{O}$.

Magnetic isotope effects of heavier elements would be certainly of great practical interest. Since effects of nuclear magnetic moments on radical pair kinetics require the absence of spin relaxation processes, which are due to other interactions involving, e.g., spin-orbit coupling, which increases strongly with nuclear charge, this seems to restrict the range of applicability of the MIE to lighter elements. So far, there seems to be no definite report on MIEs with elements heavier than oxygen, although there are indications that germanium $^{291,292,426}$ and sulfur-centered ${ }^{398}$ radicals are capable of preserving spin memory sufficiently long that hyperfine coupling effects may develop. A magnetic isotope effect reported by Molin and co-workers ${ }^{595}$ for ${ }^{117} \mathrm{Sn}$ and ${ }^{119} \mathrm{Sn}$ was disproved by them later. ${ }^{596}$

\section{Theoretical Studles}

\section{A. Mechanisms in the Gas Phase}

Cage effects in the gas phase are generally negligible. Therefore, except for the case of biradicals (cf. section IV.A), mechanisms of the paramagnetic-pair type cannot operate under gas-phase conditions. Magnetic fields may influence intramolecular radiationless transitions only, either in isolated molecules or in collision-induced processes, where intramolecular couplings provide for the mixing of different electronic manifolds and collisions provide the energy to compensate for rovibronic energy mismatch between different electronic states in small- and intermediate-size molecules. ${ }^{597-599}$ Theory of MFEs on excited-state decay of molecules in the gas phase is intimately related to the theory of radiationless processes. It will generally require a detailed consideration of molecular electronic-rovibronic states and level schemes.

Whereas in the pair mechanisms and the triplet mechanism when applied to condensed phases only spin motion is treated fully quantum mechanically, in which an effective spin Hamiltonian is used and the electronic orbital dynamic variables are treated in a phenomenological manner, the explicit Zeeman Hamiltonian is generally applied in the treatment of gas-phase MFEs.

$$
H_{\mathrm{Z}}(\boldsymbol{L}, \boldsymbol{S})=-\mu_{\mathrm{B}} \mathbf{B}_{0}(\boldsymbol{L}+2 \boldsymbol{S})
$$

Lin and Fujimura ${ }^{29}$ have considered an extended form including also diamagnetic interaction:

$H_{\mathrm{Z}}=H_{\mathrm{Z}}(\boldsymbol{L}, \boldsymbol{S})+e_{0}{ }^{2} / 8 m c^{2} \times B_{0}{ }^{2} \sum_{i}\left(x_{i}{ }^{2}+y_{i}{ }^{2}\right)$

The index $i$ runs over all electrons of the molecule. For states characterized by the quantum numbers $\left|J K M S \Sigma \Gamma_{\mathrm{el}}\right\rangle$, where $J$ is the total angular momentum, $M$ its projection onto the laboratory-fixed $z$ axis (parallel to $\mathbf{B}_{0}$ ), $K$ its projection onto the molecular axis, $S$ the electronic spin, $\Sigma$ its projection onto the molecular axis, and $\Gamma_{\mathrm{el}}$ the irreducible representation of the electronic orbital wave function, the following selection rules for magnetic coupling $\left\langle\Psi_{\mathrm{i}}\left|H_{\mathrm{Z}}\right| \Psi_{\mathrm{f}}\right\rangle$ are valid:223,600 


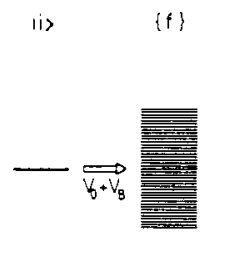

a)

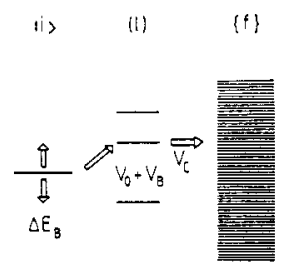

$b, c$,

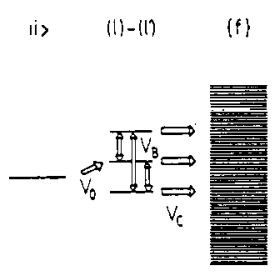

d)
Figure 42. Scheme of coupling cases serving as a basis for explaining magnetic-field-dependent decay of excited states in the gas phase; $c f$. text.

$$
\begin{gathered}
\Delta S=0 \\
\Delta J=0, \pm 1 \\
\Delta K=0, \pm 1 \\
\Delta M=0 \\
\Delta \Sigma=0 \quad \text { if } \Gamma_{\mathrm{el}, \mathrm{i}} \neq \Gamma_{\mathrm{el}, \mathrm{f}} \\
\Gamma_{\mathrm{el}, \mathrm{i}}=\Gamma_{\mathrm{el}, \mathrm{f}} \quad \text { if } \Delta \Sigma \neq 0
\end{gathered}
$$

These rules correspond to those given by de Kronig ${ }^{601}$ for spin-rotational coupling except for the extension of the $\Delta J=0$ selection rule to $\Delta J=0, \pm 1$, indicating that molecular angular momentum does not have to be conserved in an external magnetic field.

As an important consequence of the selection rule (78a) it should be noted that a magnetic field cannot directly mix states of different multiplicity $(\Delta S=0)$. Nonvanishing singlet-triplet mixing matrix elements due to Zeeman interaction may be, however, invoked in higher order perturbation theory. Thus Minaev ${ }^{602}$ advocates efficient ${ }^{1} \mathrm{n} \Pi * /{ }^{3} \mathrm{n} \Pi *$ coupling via second-order combined spin-orbit/magnetic $(L)$ coupling through the intermediacy of a $\pi \pi^{*}$ state.

Radiationless processes require that the discrete initial states are directly or indirectly coupled to a quasi-continuum. There are various types of coupling schemes that may serve to classify the mechanism of a MFE, although one should keep in mind that this mechanistic classification may be somewhat arbitrary since it depends on the choice of the quantum mechanical basis set.

Figure 42 gives a schematic illustration of the various coupling schemes considered. $|i\rangle$ denotes the initial discrete state, $\{f\}$ the final quasi-continuum, and $(l)$ or ( $l$ ) sparse manifolds of intermediate states. $v_{0}$ denotes a coupling element independent of magnetic fields whereas $v_{\mathrm{B}}$ is a coupling element proportional to the strength of the magnetic field, $v_{\mathrm{C}}$ denotes an effective average coupling element between intermediate states and final quasi-continuum (including also the effect of collisions), and $E_{B}$ is a Zeeman energy shift. The types of mechanism presented in Figure 42 summarize the rather similar models independently suggested by Atkins and Stannard, ${ }^{61,600}$ Matsuzaki and Nagakura, ${ }^{209}$ and Lin and Fujimura. ${ }^{29}$

Mechanism a entails a direct coupling between the initial state $|i\rangle$ and the manifold $\{f\}$ whereby the magnetic field may couple $|i\rangle$ and $\{f\}$ only if they are of the same multiplicity, as follows from selection rule (78a). The rate of decay of $|i\rangle$, which, according to the golden rule, is proportional to the square of the coupling matrix element $\left(v_{0}+v_{\mathrm{B}}\right)^{2}$, will depend on the sum of a linear and a quadratic term in the magnetic field $\mathbf{B}_{0}$. Orientational averaging, however, cancels the linear term. Thus, the square dependence of the magnetic field is looked upon as the characteristics of the direct mechanism, which, according to Lin and Fujimura, ${ }^{29}$ corresponds to the purely intramolecular mechanism.

In case $b$, the so-called indirect mechanism of Atkins and Stannard, ${ }^{61}$ the magnetic field perturbation couples the initial state to a sparse manifold of intermediate states that undergo final irreversible decay by collision-induced quenching. Here the magnetic-field-dependent increase of the coupling leads to an increasing contamination of the initial states with one or a few intermediate states, an effect that saturates at high field when the coupling strength exceeds the energy separation between initial and coupling intermediate states. Here, too, the magnetic field dependence starts with a $B^{2}$ behavior but soon reaches a saturation limit. It must be emphasized that, although collisions are necessary to render the process irreverisble, the mechanism of the MFE is essentially an intramolecular one.

Mechanism c, explained in the same level scheme as mechanism $b$, is based on a magnetic shift of initial or intermediate states; i.e., the Zeeman interaction is diagonal in the $|i\rangle$ and/or $(l)$ basis. In such a case the energy separation between coupling levels is variable with the magnetic field and typical resonance phenomena should occur. This type of mechanism has been termed the "energy mechanism". 600

Finally, type $d$ is also a subcase of the indirect mechanisms. It comprises a requantization of the sparse manifold $(l)$ to $(l)$, e.g., a change of quantization of the electron spin from the molecular coordinate system to the laboratory-fixed system, a phenomenon similar to the Paschen-Back effect in atomic spectroscopy. This mechanism, too, is characterized by a saturating MFD, which makes it possible to draw conclusions about the strength of the spin coupling to the molecular frame. As will be seen from the discussion below, explaining the MFD of the decay rate of $|i\rangle$ by this mechanism is by no means an obvious matter.

In what follows, we shall briefly survey the applications of these theoretical models to the experimental examples of MFEs in the gas phase outlined in section IV.A. For previous reviews on the field, cf. the articles by Küttner et al. ${ }^{24}$ and by Lin and Fujimura. ${ }^{29}$

The best-investigated case of the direct mechanism (case a) is the magnetic predissociation of iodine. The first thorough theoretical treatment of this problem was given by van Vleck, ${ }^{223}$ who showed that the emitting $\mathrm{B}^{3} \Pi_{0^{+} \mathrm{u}}$ state is magnetically coupled to the dissociative ${ }^{3} \Pi_{0-\mathrm{u}}$ state. The coupling is actually due to the different gyromagnetic ratios of orbital and spin angular momentum and bears some analogy to the $\Delta g$ mixing of $\mathrm{T}_{0}$ and $\mathrm{S}$ in radical pairs. Using the golden rule expression for the rate constant of predissociation and applying orientational averaging, the following expression was derived, valid for highly excited rotational levels:

$$
k=\frac{4 \pi^{2}}{3 h^{2}} \mu_{B}^{2} B_{0}^{2} \rho_{\mathrm{FC}}
$$

There was not enough spectroscopic information available at that time to estimate the Franck-Condon 
weighted density of states $\rho_{\mathrm{FC}}$. Such calculations were provided much later by Lin and Fujimura ${ }^{29}$ using published spectroscopic parameters on the potential curves in question. ${ }^{187,603,604}$ Thus predissociation rates for various vibrational levels $v=0-15$ could be obtained, yielding a maximum at about $v=4$. The theoretical results are in qualitative accord with data obtained experimentally by Chapman and Bunker. ${ }^{187}$

Although, for their quantitative calculation on iodine predissociation, Lin and Fujimura ${ }^{29}$ used the simplified expression of eq 79 , in their formalism they started out from a far more general expression:

$$
k=k^{(0)}+k^{(1)} B+k^{(2)} B^{2}+\ldots
$$

and provided explicit formulas for the rate coefficients $k^{(1)}$ and $k^{(2)}$. The perturbation Hamiltonian used was

$$
H^{\prime}=H_{\mathrm{nBO}}+H_{\mathrm{Z}^{\prime}}
$$

and a perturbational expansion was carried through to second order. In eq $81 H_{\mathrm{nBO}}$ denotes the non-BornOppenheimer operator and $H_{Z}{ }^{\prime}$ the nondiagonal part of the Zeeman Hamiltonian.

A new interpretation of the magnetic fluorescence quenching of iodine was necessitated by the experimental results of Vigue et al. ${ }^{190}$ on a MFD of circular polarization of the emission. The magnetic enhancement of a predissociation channel $\left(0^{+} \mathrm{u}\right) \rightarrow(1 \mathrm{u})$, which due to spin-rotational coupling, is already operating to some extent at zero field, was suggested. Due to the interference of nonmagnetic and magnetic coupling, the rate of predissociation will entail a term linear in $B_{0}$ and in the magnetic quantum $M$, causing different decay rates for different sign of $M$. Thus the predissociation will result in an orientational selection of iodine molecules, which is the reason for the occurrence of circularly polarized emission. Although this interpretation of the experimental results by the new magnetic mixing is quite convincing, it is also possible that the original channel discussed by van Vleck is operating too.

The theory of magnetic fluorescence quenching for molecules other than $\mathrm{I}_{2}$ has been theoretically treated in a more qualitative way. Further cases, where the so-called direct mechanism (case a) is invoked, are $\mathrm{NO}_{2}$ and $\mathrm{CS}_{2}$. The $\mathrm{NO}_{2}$ case shows a $B^{2}$ dependence of the quenching rate constant that is linear with pressure below $50 \mathrm{~m}$ Torr and becomes independent of pressure above 50 mTorr. Stannard ${ }^{600}$ suggested that in the pressure-saturated region the initial state is directly coupled by the magnetic interaction to a collisionally broadened continuum of the final state.

The MFD of $\mathrm{CS}_{2}$ fluorescence (cf. Figure 9) has figured as an example of a combination of the direct and indirect mechanism. ${ }^{209,600}$ This interpretation should be reconsidered in the light of conflicting recent experimental findings by Silvers et al..$^{201}$ and by Imamura et al. ${ }^{225}$ (cf. section IV.A).

The case of magnetic fluorescence quenching of glyoxal has found special interest. It is generally accepted that the magnetic field enhances the collision-induced mechanism of fluorescence quenching by radiationless singlet-triplet transitions. A direct mechanism of this kind does not operate because the singlet-triplet splitting is too small in order to provide for an effective continuum of final levels in a molecule of this size. Stannard ${ }^{600}$ attributed the magnetic-field-enhanced quenching to the increase of the number of rotational

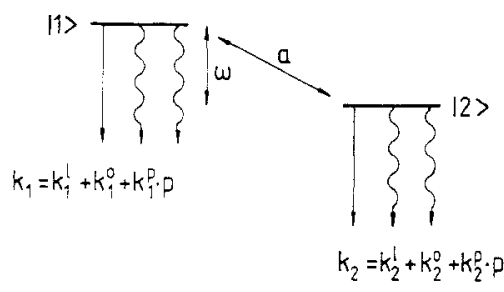

Figure 43. Two-level reaction scheme applied by Sorokin et al. ${ }^{108,203}$ to analyze MFEs on excited-state decay in the gas phase.

levels of the triplet manifold coupled to the initial states in a magnetic field. A mixing of the zero-field triplet substates by the magnetic field would not be sufficient to explain the increase of the ISC rate, since an increase in the number of coupling states is usually compensated by the decrease of coupling strength, so that the overall rate constant is invariant. However, according to Stannard,${ }^{600}$ the additional selection rule $\Delta J= \pm 1$ in a magnetic field can be made responsible for an increase of ISC in a magnetic field.

A different explanation has been offered by Schlag and co-workers, ${ }^{24,605}$ who argued that the molecular symmetry is reduced in the presence of a magnetic field. This should allow for the occurrence of an additional promoting mode of ISC in a magnetic field. The argument has, however, not been quantitatively examined.

Another model by this group of workers ${ }^{606}$ assumed the formation of a collisional complex in glyoxal fluorescence quenching. Assuming a $B^{2}$ dependence of magnetical quenching within the complex, the kinetic model could account for the observed MFD of the overall quenching rate constant. The saturation limit would be reached if each collisional complex formed is magnetically quenched.

Michel and Tric ${ }^{72}$ critically reexamined the mechanism of magnetic mixing of triplet sublevels in glyoxal. They first investigated the effect of spin-rotational coupling on the mixing of triplet substates in zero field. It was confirmed that the triplet spin substates defined in the molecular frame are only partially mixed by rotation. They are completely mixed in a magnetic field if it is stronger than the ZFS. According to the suggestion of Michel and Tric, the magnetic effect on the collision-induced overall ISC rate constant is due to a nonlinear (approximately logarithmic ${ }^{598,607}$ ) dependence of the quenching cross section on the square of the singlet-triplet mixing coefficient $\left(x^{2}\right)$. Thus the increase in the number of coupling triplet substates (three in a magnetic field, two in zero field) is not compensated by the dilution of coupling strength. This may be expressed mathematically as

$$
\begin{gathered}
k_{\mathrm{ISC}} \propto n \ln \left[x^{2}(F / n)\right] \\
k_{\mathrm{ISC}}(B) / k_{\mathrm{ISC}}(0)=3 / 2 \ln \left[x^{2}(F / 3)\right] / \ln \left[x^{2}(F / 2)\right]
\end{gathered}
$$

with $x^{2} / n$ the average mixing coefficient of a triplet substate with the initial singlet, $n$ the number of triplet substates mixed into this fluorescing singlet state, and $F$ a constant describing the efficiency of collisions. For large $x^{2} F$ the ratio $k_{\mathrm{ISC}}(B) / k_{\mathrm{ISC}}(0)$ will approach the value of 1.5 , which is close to the experimental value of 1.42 .

Sorokin et al. ${ }^{128,203}$ applied a density matrix formalism, based on a two-level scheme (cf. Figure 43) to de- 


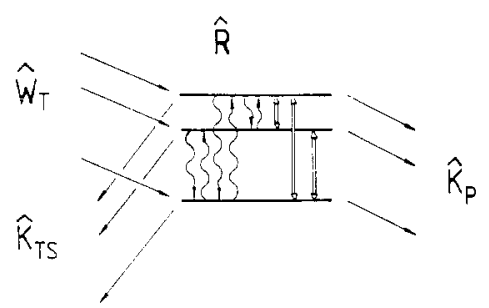

Figure 44. General scheme of the triplet mechanism. $\boldsymbol{W}_{\mathrm{T}}, \boldsymbol{K}_{\mathrm{TS}}$, and $\boldsymbol{K}_{\mathrm{P}}$ are superoperators describing sublevel-selective population, deactivation, and chemical reaction, respectively. $\boldsymbol{R}$ is the superoperator describing spin relaxation.

scribe various aspects of the MFE on yields of decay into channels of luminescence or chemical reaction for $\mathrm{H}_{2} \mathrm{CO}, \mathrm{D}_{2} \mathrm{CO}$, and $\mathrm{SO}_{2}$. The two levels represent the initially populated state $|1\rangle$ and a coupled state $|2\rangle$, each of which is characterized by the rate constants $k_{i}^{1}$ for luminescence or chemical reaction, $k_{k}^{0}$ for intramolecular radiationless decay, and $k_{i}^{p} p$ for collision-induced decay, which is proportional to the pressure $p$. Both the energy separation $(\omega)$ and the coupling strength $(a)$ may be linear functions of the magnetic field. Taking also into account a collision-induced phase relaxation rate $k_{3}^{p} p$ for states $|1\rangle$ and $|2\rangle$, the stationary solution of the stochastic Liouville equation after primary excitation of state $|1\rangle$ leads to the following expression for the yield of fluorescence from state $|1\rangle$ :

$\varphi_{1}^{1}=k_{1}^{1} \times \frac{k_{2}\left(\omega^{2}+k_{12}^{2}\right)+2 a^{2} k_{12}}{k_{1} k_{2}\left(k_{12}{ }^{2}+\omega^{2}\right)+2 a^{2} k_{12}\left(k_{1}+k_{2}\right)}$

where $k_{12}$ is given by

$$
k_{12}=2\left(k_{1}+k_{2}\right)+k_{3}^{P}
$$

The result has been useful for discussing various cases (e.g., magnetic-field-dependent $\omega$ and/or $a$ ) in a semiempirical way. In fact, various features of the MFEs are consistent with this general relation.

\section{B. The Triplet Mechanism}

A general kinetic framework for theoretical treatments of the triplet mechanism is provided in Figure 44. ${ }^{160}$ The choice of the triplet substates $\mathrm{T}_{\alpha}, \mathrm{T}_{\beta}$, and $\mathrm{T}_{\gamma}$, used to describe the dynamical situation, is not unique and is suggested by the way one prefers to visualize the process in a semiclassical picture. Of course, the underlying physics must be independent of the basis set chosen. In general, $\mathrm{T}_{\alpha}, \mathrm{T}_{\beta}$, and $\mathrm{T}_{\gamma}$ are not identical with the actual eigenfunctions of the spin Hamiltonian $H$ in an arbitrary external magnetic field, but rather with the eigenstates of some limiting-case spin Hamiltonian $H_{0}$ (e.g., zero field $\left(H_{\mathrm{D}}\right)$ or high field $\left(H_{\mathrm{Z}}\right)$. Then, there will be coherent transitions among the triplet substates due to their coupling by $\left(H-H_{0}\right)$, which is indicated by the "resonance" arrows in the scheme of Figure 44. Furthermore, due to a stochastic modulation of $H$, especially by the tumbling motion of the molecule, there will be incoherent transitions among the substates, represented by wavy arrows and theoretically dealt with by a superoperator $\boldsymbol{R}$ acting on the triplet spin density matrix $\rho$. Sublevel-selective population is represented by the operator $W_{\mathrm{T}}$, and chemical decay and ISC to the singlet ground state are represented by the operators $K_{\mathrm{P}}$ and $K_{\mathrm{S}}$, respectively. A general equation of motion for the triplet spin density matrix is given by $(\hbar=1)$

$$
\rho=-i[H, \rho]_{-}-\boldsymbol{R}_{\rho}-1 / 2\left[K_{\mathrm{P}}+K_{\mathrm{S}}, \rho\right]_{+}+W_{\mathrm{T}}
$$

where [ ] and [ $]_{+}$denote the commutator and anticommutator, respectively. From the solution $\rho(t)$ the yield into the product channel is obtained according to

$$
\Phi_{\mathrm{P}}=\operatorname{Tr} \int_{0}^{\infty} K_{\mathrm{P}} \rho \mathrm{d} t
$$

It may be convenient to represent the spin density matrix in terms of one of the following basis sets, which are eigenfunctions of the corresponding spin Hamiltonians:

$$
\begin{gathered}
\left\{\mathrm{T}_{x}, \mathrm{~T}_{y}, \mathrm{~T}_{z}\right\} \equiv(x y z): H_{\mathrm{D}} \\
\left\{\mathrm{T}_{\mathrm{u}}, \mathrm{T}_{\mathrm{c}}, \mathrm{T}_{\mathrm{l}}\right\} \equiv(\mathrm{ucl}): H_{\mathrm{D}}+H_{\mathrm{Z}} \\
\left\{\mathrm{T}_{+}, \mathrm{T}_{0}, \mathrm{~T}_{-}\right\} \equiv(+0-): H_{\mathrm{Z}}
\end{gathered}
$$

Spin-sublevel-selective population of the triplet in zero field is described by an operator $W_{\mathrm{T}}$, diagonal in the molecule-fixed $(x y z)$ basis:

$$
W_{\mathrm{T}}=w_{\mathrm{T} x} P_{x}+w_{\mathrm{T} y} P_{y}+w_{\mathrm{T} z} P_{z}
$$

where $P_{x}, P_{y}$, and $P_{z}$ are projection operators, projecting onto the respective spin states and $w_{\mathrm{T} x}, w_{\mathrm{T} y}$, and $w_{\mathrm{T} z}$ are corresponding single-level population rates. In triplet-state ODMR spectroscopy ${ }^{561}$ and in the first versions of the CIDEP triplet mechanism ${ }^{15,79}$ the operator $W_{\mathrm{T}}$ was assumed to be diagonal in the (ucl) basis when a magnetic field is applied

$$
W_{\mathrm{T}}^{B}=\sum_{\alpha=\mathrm{u}, \mathrm{c}, 1} w_{\mathrm{T} \alpha} P_{\alpha}
$$

with

$$
w_{\mathbf{T} \alpha}=\sum_{i=x, y, z}\left|c_{\alpha, i}\right|^{2} w_{\mathbf{T} i}
$$

where the $c_{\alpha, i}$ are the elements of the transformation matrix relating the $(x y z)$ to the (ucl) basis. One should note that the transition from eq 89 to eq 91 does not simply correspond to a change of the basis, but, in addition, to a neglect of the off-diagonal density matrix elements after the basis transformation. Actually, this point is essential in order that spin polarization can result when, in a final step, the density matrix is projected onto the (+0-) basis, appropriate to describe the spin state after dissociation of the triplet into a radical pair. Projecting, instead, in a nontruncated way directly from the selectively populated $(x y z)$ basis of the triplet onto the $(+0-)$ basis of the radical pair would produce no spin polarization. Schematically the spin polarization mechanism may be expressed by the following shorthand notation:

$$
W_{\mathrm{T}}(\text { diag })_{x y z} \underset{\substack{\text { diagonal } \\ \text { truncation }}}{\stackrel{x y z \rightarrow \text { ucl }}{\longrightarrow}} \rho(\text { diag })_{\text {ucl }} \frac{\text { ucl } \rightarrow+0-}{\substack{\text { diagonal } \\ \text { truncation }}} \rho(\text { diag })_{+0-}
$$

It has been pointed out by El-Sayed and Leyerle ${ }^{608}$ that, if singlet-triplet intersystem crossing populates $T_{1}$ indirectly via $T_{2}$, the polarization could be different from the case where $T_{1}$ is populated directly, because the ZFS parameters may be different in the two electronic states. 
The central part of scheme 92 would then have to be replaced by

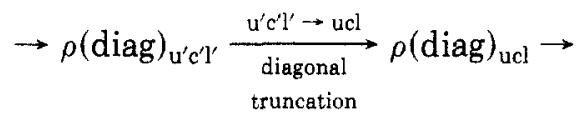

In the more advanced theories of the triplet mechanism as applied to CIDEP ${ }^{609-611}$ or to MFEs on product yields ${ }^{80,160,335,612}$ the spin-sublevel-selective population or depopulation term is assumed to be diagonal in the $(x y z)$ basis independent of the strength of the external magnetic field. Then it may be shown, as has been nicely illustrated with a vector model by Hore,${ }^{613}$ that spin polarization is not produced instantaneously, but requires a time span in the order of one Larmor precession period before the nondiagonal elements in the (ucl) basis have decayed by phase randomization.

Felix and Weissman ${ }^{614}$ raised the question of a possible interference of $\left\langle\mathrm{S}_{1}\left|H_{\mathrm{soc}}\right| \mathrm{T}_{1 x(y z)}\right\rangle$ coupling elements in a magnetic field when the $(x y z)$ substates are recoupled to (ucl) states. Applying the golden rule expression, one would relate the zero-field rates $w_{\mathrm{T} i}(i=$ $x, z)$ to the field-dependent rates $w_{\mathrm{T} \alpha}(\alpha=\mathrm{ucl})$ by an expression of the type

$$
w_{\mathrm{T} \alpha}=\sum_{i=x, y, z}\left|c_{\alpha, i}\right|^{2} w_{\mathrm{T}, i}+\sum_{i \neq j} c_{\alpha, i} c_{\alpha, j}^{*}\left(w_{\mathrm{T}, i} w_{\mathrm{T}, j}\right)^{1 / 2}
$$

The analysis of triplet population and depopulation kinetics measured by Felix and Weissman did not support the necessity to take interference terms, corresponding to the second sum in eq 94 , into account.

As with the radical pair mechanism, theories of triplet mechanism type CIDEP and MFEs on product yields are closely related. Whereas, however, CIDEP effects may arise from both spin-sublevel-selective popula$\operatorname{tion}^{15,79}$ and/or depopulation ${ }^{615,616}$ of a reactive triplet state, MFEs on product yields will ensue only in the case of spin-selective depopulation. ${ }^{80,325}$ The kinetic treatment of the triplet mechanism under conditions of isolated triplet sublevels (i.e., negligible spin-lattice relaxation) at low temperatures is straightforward and has been applied in several cases to experiments probing the triplet lifetime. ${ }^{228,280,484,618,619}$ Astonishingly, except for some qualitative speculations published in 1972 by Gupta and Hammond ${ }^{326,624}$ on a possible participation of this type of mechanism in triplet-sensitized cis-trans isomerizations of olefins, the principles of the triplet mechanism were not applied before $1980^{80,335}$ to explain MFEs on product yields in liquid solutions, several years after profound theories of triplet mechanism type CIDEP had already been developed.

The first dynamical treatment of the triplet mechanism as applied to CIDEP in liquid solution was worked out by Atkins and Evans. ${ }^{609,617}$ Their treatment was based on the following stochastic Liouville equation:

$\rho=-i\left[H_{\mathrm{Z}}+H_{\mathrm{D}}(\Omega), \rho\right]_{-}-k_{\mathrm{p}} \rho+K_{\mathrm{T}}(\Omega) \exp \left(-k^{\prime} t\right)$

The triplet spin density matrix $\rho$ is represented in a laboratory-fixed system of reference and therefore the ZFS operator $H_{\mathrm{D}}$ is a function of the molecular orientation, represented by the set of Eulerian angles $\Omega(t)$, which undergo stochastic changes due to the rotational diffusion of the molecule. Since spin relaxation is mainly due to this stochastic process, an explicit expression separately accounting for spin relaxation does not appear at this stage of the theory. The rate con- stant $k_{\mathrm{p}}$ characterizes the non-sublevel-selective decay of the triplet to radicals, wherein the spin polarization is to be probed. In eq 95 only sublevel-selective triplet population is considered. The source term is proportional to the concentration of decaying excited singlets $\left(\exp \left(-k^{\prime} t\right)\right)$ and its sublevel selectivity is described by a rate constant operator $K_{\mathrm{T}}(\Omega)$, which is assumed to be diagonal in the same molecule-fixed basis as $H_{\mathrm{D}}(\Omega)$. The ZFS Hamiltonian $H_{\mathrm{D}}(\Omega)$ has been represented by

$$
H_{\mathrm{D}}(\Omega)=-\mathbf{S D}(\Omega) \boldsymbol{S}
$$

where $\mathbf{D}(\Omega)$ is a traceless tensor that may be characterized by constants $D$ and $E$ related to the diagonal elements in the main axis system by

$$
\begin{gathered}
D=1 / 2\left(D_{x x}+D_{y y}\right)-D_{z z} \\
E=-1 / 2\left(D_{x x}-D_{y y}\right)
\end{gathered}
$$

It has been convenient to introduce irreducible tensor notation (for details of this formalism, cf. the books by Fano and Racah, ${ }^{620}$ Edmonds, ${ }^{621}$ and Rose ${ }^{622}$ ) whereby eq 96 adopts the following form:

$$
H_{\mathrm{D}}(\Omega)=\sum_{Q=-2}^{2}(-1)^{Q} D_{Q}{ }^{(2)}(\Omega) S_{-Q}{ }^{(2)}
$$

The components of the second-rank tensor operator $\boldsymbol{S}^{(2)}$ are defined by eq 99 . (Note that there are some misprints in ref 609.)

$$
\begin{gathered}
S_{0}^{(2)}=(3 / 2)^{1 / 2}\left(S_{z}{ }^{2}-S^{2} / 3\right) \\
S_{ \pm 1}{ }^{(2)}=\mp 1 / 2\left(S_{z} S_{ \pm}+S_{ \pm} S_{z}\right) \\
S_{ \pm 2}{ }^{(2)}=1 / 2 S_{ \pm} S_{ \pm}
\end{gathered}
$$

The coefficients $D_{Q}{ }^{(2)}(\Omega)$ are given by

$$
D_{Q}^{(2)}(\Omega)=(2 / 3)^{1 / 2} D D_{0, Q}^{(2)}(\Omega)+E\left(D_{2, Q}^{(2)}(\Omega)+D_{-2, Q}^{(2)}(\Omega)\right)
$$

The angular dependence is expressed through the elements of the Wigner rotational matrices $D_{Q, Q}^{(2)}(\Omega)$ (cf. ref 620-622).

The spin-selective kinetic operator $K_{\mathrm{T}}(\Omega)$ is expressed in a form isomorphic with eq 98 , but including an additional isotropic contribution:

$$
K_{\mathrm{T}}(\Omega)=1 / 6 k_{\mathrm{T}} \boldsymbol{S}^{2}+\sum_{Q=-2}^{2}(-1)^{Q} K_{Q}{ }^{(2)}(\Omega) S_{-Q}{ }^{(2)}
$$

where $k_{\mathrm{T}}$ is given by

$$
k_{\mathrm{T}}=1 / 3\left(k_{\mathrm{T} x}+k_{\mathrm{T} y}+k_{\mathrm{T} z}\right)
$$

The function $K_{Q}{ }^{(2)}(\Omega)$ is of the form of eq 100 but with $D$ and $E$ replaced by $D_{k}$ and $E_{k}$, given by

$$
\begin{gathered}
D_{k}=1 / 2\left(k_{\mathrm{T} x}+k_{\mathrm{T} y}\right)-k_{\mathrm{T} z} \\
E_{k}=-1 / 2\left(k_{\mathrm{T} x}-k_{\mathrm{T} y}\right)
\end{gathered}
$$

In order to obtain an approximate analytical solution for the ensemble-averaged time-integrated density matrix, eq 95 was transformed to the rotating frame and converted into an integrodifferential equation by formal integration and resubstitution of the result into the differential equation. Ensemble averaging was performed by applying the fast motional approximation 
(i.e., orientational correlation time much shorter than the Larmor period or the triplet decay time). Time integrating and sample averaging required evaluation of Laplace transforms of second-rank tensor time correlation functions, yielding a linear matrix equation for the time-integrated, ensemble-averaged density matrix from which analytical expressions can be obtained for any observable of interest (after complete triplet decay). Whereas Atkins and Evans were interested in spin polarization, ${ }^{609}$ only recently has their method been adapted by Serebrennikov and Minaev ${ }^{612}$ to the problem of magnetic-field-dependent product yields. As noted above, this requires introduction of spin-sublevel-selective triplet decay. Serebrennikov and Minaev used a selective operator of triplet deactivation $K_{\mathrm{S}}(\Omega)$, defined analogously to eq 101-103. On the other hand, the triplet production process and the product formation from the triplet were assumed to be non sublevel selective. The result obtained for the relative MFD of the product quantum yield is ${ }^{612}$

$R\left(\Phi_{\mathrm{P}}, \omega_{0}\right)=-\frac{2}{3}\left(\frac{\omega_{0}}{k_{1}}\right)^{2} \frac{D_{k}{ }^{2}+3 E_{k}{ }^{2}}{D^{2}+3 E^{2}} \frac{1}{1+k_{0} T_{1}}$

with $T_{1}$ given by

$$
\begin{aligned}
& T_{1}^{-1}= \\
& \quad \frac{2}{15 k_{1}}\left(D^{2}+3 E^{2}\right)\left(\frac{1}{1+\left(\omega_{0} / k_{1}\right)^{2}}+\frac{4}{1+4\left(\omega_{0} / k_{1}\right)^{2}}\right)
\end{aligned}
$$

and the definitions

$$
\begin{gathered}
k_{1}=k_{0}+\tau_{\theta 2}{ }^{-1} \\
k_{0}=k_{\mathrm{p}}+k_{\mathrm{S}} \\
k_{\mathrm{S}}=1 / 3\left(k_{\mathrm{T} x \mathrm{~S}}+k_{\mathrm{T} y \mathrm{~S}}+k_{\mathrm{T} z \mathrm{~S}}\right) \\
\omega_{0}=\mu_{\mathrm{B}} B_{0} / \hbar \\
D_{k^{\prime}}=1 / 2\left(k_{\mathrm{T} x \mathrm{~S}}+k_{\mathrm{T} y \mathrm{~S}}\right)-k_{\mathrm{T} z \mathrm{~S}} \\
E_{k^{\prime}}=-1 / 2\left(k_{\mathrm{T} x \mathrm{~S}}-k_{\mathrm{T} y \mathrm{~S}}\right)
\end{gathered}
$$

The meaning of the rate constants follows from Figure 44. The correlation time $\tau_{\theta 2}$ for a second-rank tensor equals $6 D_{\mathrm{r}}$ in the case of isotropic rotational diffusion, where $D_{\mathrm{r}}$ is the rotational diffusion coefficient.

The result of eq 104 has been also derived in a more direct way, using the "strong collisional" approach, i.e., a random molecular motion, whereby in one "collision" occurring after an average time of $\tau_{\mathrm{R}}$ the orientational distribution acquires statistical equilibrium. In this case the Laplace transform of $\rho$ may be obtained in an explicit formal solution that leads to exact closed-form expressions for the quantum yield in the zero-field and the high-field limits. The result (104) for general field is obtained from a series expansion accurate to second order in a parameter $\epsilon$, which may be approximated as

$$
\epsilon \approx\left(|D|+\left|D_{k}\right|\right) / k_{1}
$$

As noted by Serebrennikov and Minaev ${ }^{612}$ the condition

$$
\epsilon^{2} \ll 1
$$

is tantamount to small magnetic field effects $\left(R\left(\Phi_{\mathrm{P}}\right)\right)$.
When eq 104 is applied to the thionine/p-iodoaniline triplet exciplex decay investigated by Steiner and coworkers, ${ }^{160}$ where the saturation value of the $\mathrm{MFE}$ amounts to approximately $-20 \%$ and the parameters correspond to $\epsilon \approx 2 / 3$, the results according to eq 104 deviate by up to a factor of 2 from the exact solution. ${ }^{612}$

Pedersen and Freed ${ }^{611}$ treated the triplet-mechanism CIDEP problem by a numerical method that is quite general and applies also to the slow-motional region of the parameters. Their starting equation has been modified by Steiner and co-workers ${ }^{160}$ by including a term for spin-sublevel-selective triplet decay in order to account also for MFEs on product yields:

$$
\begin{array}{r}
\dot{\rho}(\Omega, t)=-i\left[H_{\mathrm{Z}}+H_{\mathrm{D}}(\Omega), \rho(\Omega, t)\right]_{-}-1 / 2\left[K_{\mathrm{S}}(\Omega), \rho(\Omega, t)\right]_{+}- \\
k_{\mathrm{p}} \rho(\Omega, t)-\Gamma_{\Omega} \rho(\Omega, t)(109)
\end{array}
$$

Here the density matrix $\rho(\Omega, t)$ is an explicit function of the set of orientational angles $\Omega$, and $\Gamma_{\Omega}$ is a Markovian operator describing the change of orientational distribution due to molecular tumbling. ${ }^{623}$ Tripletsublevel-selective population is accounted for by suitable initial conditions for the density matrix.

In the case of continuous rotational diffusion, the Wigner rotational matrix elements $D_{Q, Q}^{(L)}(\Omega)$ are eigenfunctions of $\Gamma_{\Omega}$, where, in the special case of isotropic rotational diffusion, we have

$$
\Gamma_{\Omega} D_{Q^{\prime}, Q^{\prime}}^{(L)}(\Omega)=D_{\mathrm{r}} L(L+1) D_{Q^{\prime} Q^{\prime}}^{(L)}(\Omega)
$$

with $D_{\mathrm{r}}$ the rotational diffusion coefficient.

The time-integrated solution to eq 109 is obtained by Laplace transformation, letting $s \rightarrow 0$ and expanding the Laplace transform $\rho(\Omega, 0)$ in a series of the Wigner rotation matrix elements, representing a complete orthonormal set in the space of Eulerian angles.

$$
\rho(\Omega, 0)=\sum C_{Q, Q^{(L)}}^{(L)} D_{Q, Q^{\prime}}^{(L)}(\Omega)
$$

The coefficients $C_{Q, Q}^{(L)}$, are themselves $3 \times 3$ matrices and may be obtained from the numerical solution of an infinite system of linear equations derived from eq 109 by making use of the orthonormal properties of the functions $D_{Q, Q}^{(L)}$. The infinite system of equations is truncated at some order, sufficient to guarantee convergence of the solution. Actually, the final physical information is extracted from the angular average of $\rho(\Omega, 0)$, which is identical with the zeroth-order matrix $C_{0,0}^{(0)}$. Thus, e.g., the product yield is given by

$$
\Phi_{\mathrm{P}}=k_{\mathrm{P}} \mathrm{T}_{\mathrm{r}}\left\{C_{0,6}^{(0)}\right\}
$$

Numerical solutions to eq 109 for various sets of parameter values have been obtained by Steiner and coworkers, ${ }^{160}$ who used these results for the analysis of experimental MFEs with heavy-atom-substituted triplet exciplexes. Some typical results are depicted in Figure 45.

Steiner ${ }^{80,160,335}$ has also derived an approximate analytical expression for the magnetic-field-dependent quantum yield, approaching the problem from an intuitive kinetic point of view. Here the zero-field triplet substates are considered as the appropriate kinetic basis throughout, and all processes, including the coherent transitions among these states induced by a magnetic field, are approximated as first-order rate processes connecting or depleting the triplet substates. Calculation of the quantum yield within such a kinetic scheme is then a straightforward matter. 


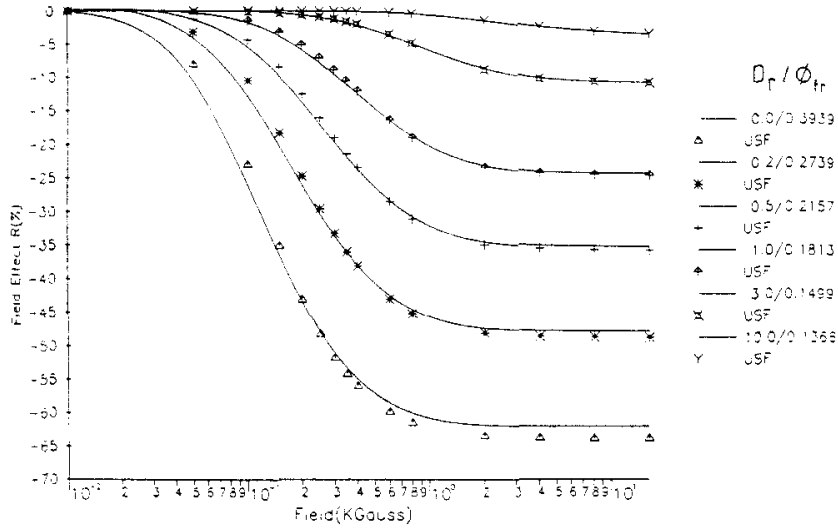

Figure 45. Results of model calculations ${ }^{775}$ on the triplet mechanism including sublevel-selective depopulation only. $\mathrm{Pa}$ rameters are $D, E=0, k_{\mathrm{S}}=20 / 3, D_{k}^{\prime}=10, E_{k}{ }^{\prime}=0$, and $k_{\mathrm{P}}=1$. $D_{r}$ is varied as indicated in the figure. $\Phi_{\mathrm{fr}}$ values given are absolute product yields in zero field. Solid lines: results from numerical solution of SLE, 109. Data points: results from approximation (USF), given by eq 118 .

In order to connect the quantum mechanical spin motion to the kinetic scheme, spin evolution within the triplet is described in terms of an autocorrelation function of the electron spin in the molecular frame, developing stochastically as well as coherently due to the combined action of rotational diffusion and Larmor precession of the spin about the external magnetic field. For the case of isotropic rotational diffusion (diffusion constant $D_{\mathrm{r}}$ ) and neglecting the ZFS, the probability $p\left(\mathrm{~T}_{\alpha^{\prime}}, t \mid \mathrm{T}_{\alpha}, 0\right)$ of finding the molecule in a specific substate $\mathrm{T}_{\alpha^{\prime}}\left(\alpha^{\prime}=x, y, z\right)$ at time $t$, if it was $\mathrm{T}_{\alpha}$ at time $t$ $=0$ (with a random orientational distribution, however), is given by

$$
\begin{aligned}
& p\left(\mathrm{~T}_{\alpha^{\prime}}, t \mid \mathrm{T}_{\alpha}, 0\right)= \\
& 1 / 3+2 / 15 \exp \left(-6 D_{\mathrm{r}} t\right)\left\{1+2 \cos \left(\omega_{0} t\right)+2 \cos \left(2 \omega_{0} t\right)\right\}
\end{aligned}
$$

This function describes an exponential/oscillatory approach to spin equilibrium. The spin motion may be incorporated into the kinetic reaction scheme by relating it to an equivalent transition rate constant $k\left(\mathrm{~T}_{\alpha}\right.$ $\rightarrow \mathrm{T}_{\alpha^{\prime}}$ ):

$$
k\left(\mathrm{~T}_{\alpha} \rightarrow \mathrm{T}_{\alpha^{\prime}}\right)=2 D_{\mathrm{r}, B}
$$

where $D_{\mathrm{r}, B}$ is given by

$$
D_{\mathrm{r}, B}=\frac{s\left(1-p_{\mathrm{S}}\right)}{6 s p_{\mathrm{S}}-2}
$$

with $p_{\mathrm{S}}$ the Laplace transform of eq 113:

$$
\begin{aligned}
p_{\mathrm{S}}=\frac{1}{15}\left(\frac{5}{s}+\frac{2}{s+6 D_{\mathrm{r}}}+\frac{4\left(s+6 D_{\mathrm{r}}\right)}{\left(s+6 D_{\mathrm{r}}\right)^{2}+\omega_{0}^{2}}+\right. \\
\left.\frac{4\left(s+6 D_{\mathrm{r}}\right)}{\left(s+6 D_{\mathrm{r}}\right)^{2}+4 \omega_{0}^{2}}\right)
\end{aligned}
$$

The best fit to the results of exact numerical solutions is obtained by setting

$$
s=k_{0}=k_{\mathrm{p}}+k_{\mathrm{S}}
$$

with $k_{\mathrm{S}}$ defined by eq $106 \mathrm{c}$.

In zero field the decay of the autocorrelation function is purely exponential and $D_{\mathrm{r}, B}$ equals $D_{\mathrm{r}}$, the rotational diffusion coefficient. In this case the procedure yields the exact result. The quantum yield is obtained from the kinetic scheme as outlined above. In the special case where $k_{T x \mathrm{~S}}=k_{\mathrm{TyS}}$, i.e., $E_{k}=0$, the quantum yield $\Phi_{\mathrm{P}}$ of magnetic-field-dependent product formation from the triplet is

$$
\begin{array}{r}
\Phi_{\mathrm{P}}=k_{\mathrm{p}}\left(k_{\mathrm{p}}+k_{\mathrm{S}}-D_{k} / 3+6 D_{\mathrm{r}, B}\right) /\left(( k _ { \mathrm { p } } + D _ { k } ) \left(k_{\mathrm{p}}+\right.\right. \\
\left.\left.4 D_{\mathrm{r}, B}\right)+2 k_{\mathrm{p}} D_{\mathrm{r}, B}+\left(3 k_{\mathrm{S}}-2 D_{k}\right)\left(k_{\mathrm{p}}+D_{k}+2 D_{\mathrm{r}, B}\right)\right\}
\end{array}
$$

Note that in this review many expressions may differ from those in the original work because they were adapted in order that they comply with a standardized notation throughout this review.

As was noted above, the ZFS of the triplet substates has been neglected in deriving the spin autocorrelation function. This precludes of course evaluation of spin polarization. However, magnetic field effects on product yields from the triplet state are in excellent agreement with the exact numerical solutions (cf. Figure 45) if

$$
D, E \ll k_{\mathrm{p}}+k_{\mathrm{g}}+6 D_{\mathrm{r}}
$$

Comparing this condition with the $\epsilon$-condition of Serebrennikov and Minaev, ${ }^{612}$ one can show that condition 119 is compatible with $\epsilon=3 / 2$ and has a wider range of applicability than eq 104.

When the ZFS approaches or exceeds the limit of eq 119 , this leads to an increase of the $B_{1 / 2}$ value, since an external magnetic field can decouple the spin motion from the molecular frame only if $B \geq D$. As may be seen from the numerical solution, however, the limiting high-field magnetic field effect is independent of ZFS, ${ }^{160}$ and eq 118 again provides an acceptable solution in the high-field limit, even if condition 119 is not obeyed.

\section{The Radical Pair Mechanism}

\section{Historical Roots}

Although the development of the radical mechanism has been intimately related to the discovery and early investigations of CIDNP and CIDEP phenomena, it is worth mentioning that the essential principle of this mechanism was established about 15 years before by physicists in order to explain and evaluate the magnetic field dependence of positronium decay. ${ }^{625-627}$ Positronium, an exotic atom composed of an electron and a positron, decays in an antiparticle annihilation process, whereby two or three photons are emitted, depending on whether the total spin of positronium is zero (para form, $F=0$ ) or 1 (ortho form, $F=1$ ). The former process is about 1000 times faster than the latter, so that the lifetime of the ortho form is correspondingly longer. Due to the different $g$ factors of electron and positron $(\Delta g=4)$, the $m=0$ component of the ortho state is strongly coupled to the para state in a magnetic field, whereby the lifetime of this ortho-state component is significantly decreased. The quantitative theory of static as well as of resonant MFEs was treated in complete analogy to the $\Delta g$ mechanism in a radical pair of fixed distance. ${ }^{626}$ Later, the principle of this mechanism was adapted by Frankevich ${ }^{284,628}$ to account for MFEs on electron-hole recombination (lifetime of Wannier excitons) in molecular crystals. However, this work does 
not seem to have had any impact on the development of the radical pair mechanism in chemical systems.

Nuclear spin polarization in the course of a chemical reaction was first seen by Bargon, Fischer, and Johnson $^{86}$ and by Ward and Lawler. ${ }^{87}$ The effects were first thought to be due to a transfer of spin polarization from radical electron spin to nuclear spins due to the Overhauser mechanism in single radicals. ${ }^{629,630}$ Soon, however, it became clear that special features of the polarization pattern, e.g., the multiplet effect, could not be accounted for by any refinement of the Overhauser mechanism.

The basic concept of radical pairs with their correlated spin motion as the final cause of electronic and nuclear spin polarization was suggested independently by Kaptein and Oosterhoff ${ }^{84,631}$ and by Closs. ${ }^{632}$ Their first treatments concentrated on the high-field case, where only $\mathrm{S}-\mathrm{T}_{0}$ mixing occurs in radical pairs. Whereas Closs ${ }^{632}$ in his first paper treated $\mathrm{T}_{0}-\mathrm{S}$ transitions in a radical pair with two nuclear spins $I=1 / 2$ in a noncoherent fashion, characterizing the evolving nuclear spin polarization as a transverse Overhauser effect, which brings about equal intensities of emission and absorption within the multiplet of lines, the treatment by Kaptein and Oosterhoff ${ }^{84,631}$ introduced the notion of coherent spin motion in a situation where hyperfine coupling energies are comparable to the exchange interaction $J$ in a radical pair. Common to both suggestions $\mathrm{s}^{631,632}$ was the idea that a sorting of nuclear spin orientations was achieved by the spin selectivity of chemical recombination in the radical pair. This spin-selective cage reaction competes with diffusive separation of the radicals leading to products, which may differ from those of cage recombination. The role of $g$-factor difference in a radical pair for inducing singlet-triplet transitions in a magnetic field and causing net nuclear spin polarization was first demonstrated by Gerhart ${ }^{633}$ and by Closs and Trifunac. ${ }^{634}$

Finally, the idea that diffusion and geminate reencounters of radical pairs are of essential importance was contributed by Adrian, ${ }^{635-637}$ who treated this problem in terms of Noyes' ${ }^{638}$ theory of geminate radical pair reencounter statistics. Thus, a few years after the discovery of CIDNP phenomena all the essential ingredients of the radical pair mechanism had been assessed. The rigorous theoretical formalism was developed during the 1970s.

Consequences of the radical pair mechanism pertaining to the magnetic sensitivity of chemical yields or magnetic isotope effects were fist discussed by Lawler and Evans, ${ }^{94}$ and, in fact, the first effect of this kind was systematically revealed by Molin and co-workers ${ }^{137}$ in systems where CIDNP effects were already known to occur. ${ }^{293}$ A quantitative analysis of these first effects was already based on the radical pair mechanism. ${ }^{639}$

In tracing back early theoretical roots of MFEs on radical reactions, we must mention two other contributions. Brocklehurst ${ }^{397}$ considered the implications of electron spin relaxation on the MFD of radical pair recombination products, mainly with the situation in radioluminescence in mind. Although such mechanisms turned out to be of minor importance for low-viscosity homogeneous solution systems, they are of basic importance for long-lived geminate radical pairs, e.g., those occurring in micellar systems.
Further, it is of interest that, quite independent of the CIDNP radical pair mechanism, Merrifield and co-workers ${ }^{465}$ invoked exactly this radical pair mechanism to explain the magnetic modulation of dye-sensitized delayed fluorescence in an organic crystal. They provided a quantitative model treatment on the basis of a stochastic Liouville equation for a radical pair with one nuclear spin $I=1 / 2$.

The radical pair mechanism has been the subject of an appreciable number of review articles (cf. Table 1). Most of them have dealt with applications to CIDNP,, 11 CIDEP, ${ }^{20,51}$ or CIDMP in general. ${ }^{10,18,23,28,30,35}$ With the exception of reviews especially devoted to the primary radical pair in photosynthetic reaction centers, ${ }^{37,45}$ reviews of the radical pair mechanism as involved in magnetic field effects on chemical yields or kinetics seem to have been a special domain of Russian authors. ${ }^{19,22,31,50,52}$ So far, the treatise by Salikhov et al. ${ }^{52}$ gives the most comprehensive account of the subject. Of course, in their monograph the theoretical contributions of the authors are particularly emphasized, although due credit is given to the work of others.

In this review our prime goal will be to provide a compact, yet comprehensive, survey of the relevant theoretical work done so far, compiling and ordering the various contributions from a methodological point of view. We think that providing such a general schematic in addition to the more specialized reviews cited above will be useful for a quick introduction and for more easily assessing novel contributions to the field.

\section{General Formalism}

The quantities of interest to the experimentalist are chemical yields $Y_{\mathrm{S}}(t)$ and $Y_{\mathrm{T}}(t)$ of recombination products formed in either the singlet or triplet state, respectively, or yields of products arising from scavenging reactions of the radical $Y_{\mathrm{SC}}(t)$. These quantities are identical with or directly related to the yields and quantum yields in the experimental section. In most experimental applications only the limiting value for $t \rightarrow \infty$, here symbolized as $Y_{\mathrm{S}}, Y_{\mathrm{T}}$, and $Y_{\mathrm{SC}}$, is considered. In CIDNP experiments it is necessary to know $Y_{\mathrm{S},(\mathrm{n})}$ and $Y_{\mathrm{SC},(\mathrm{n})}$, the corresponding product yields assigned to the specific nuclear configurations (n). Of course, any theory supplying these quantities will be likewise suitable to obtain $Y_{\mathrm{S}}, Y_{\mathrm{T}}$, and $Y_{\mathrm{SC}}$ characterizing the MARY and RYDMR effects, since these are merely the averages of the corresponding quantities over various nuclear states. Therefore, although CIDNP effects in themselves are not the subject of this review, theoretical work in this field will necessarily have a bearing on the objectives of it and will be considered, too, in this section, as far as the general problems of the radical pair mechanism are concerned.

The dynamic variables necessary to yield a satisfactory description of the radical pair kinetics comprise the electronic $\left(\boldsymbol{S}_{i}\right)$ and nuclear spins $\left(\boldsymbol{I}_{j}\right)$, the radical pair separation ( $\mathbf{r}$ ), and the relative orientation of the radicals $(\Omega)$. Whereas the spins have to be treated quantum mechanically, radical separation and orientation are usually treated as classical stochastical variables. Thus the state of the radical pair is conveniently described by an electron-nuclear spin density matrix $\rho$ $(\mathbf{r}, \Omega, t)$. An equation of motion of the spin density 
matrix representing the relevant processes in a radical pair is given in the following generalized stochastic Liouville equation (SLE):

$$
\rho(\mathbf{r}, \Omega, t)=-i[H(\mathbf{r}, \Omega), \rho(\mathbf{r}, \Omega, t)]_{-}+\Gamma(\mathbf{r}, \Omega) \rho(\mathbf{r}, \Omega, t)+
$$$$
\boldsymbol{K}(\mathbf{r}, \Omega) \rho(\mathbf{r}, \Omega, t)
$$

Here [ ] denotes the commutator. The spin Hamiltonian $H(\mathbf{r}, \Omega)$ describes the coherent spin motion of radical pairs separated by $\mathbf{r}$ and oriented as specified by $\Omega$. The stochastic operator $\Gamma(\mathbf{r}, \Omega)$ describes the random translational and rotational diffusion of the radical pair. This form of description of the motion of quantum systems coupled to a bath via stochastic classical degrees of freedom has been established and justified by Kubo. ${ }^{640}$

The electron-spin-dependent chemical change of the radical pair is expressed by the last term in eq 120 . Here $\boldsymbol{K}(\mathbf{r}, \Omega)$ represents a general linear superoperator in electron-nuclear spin Liouville space. In general, it may depend on the interradical separation and the relative radical orientations. The operator $\boldsymbol{K}(\mathbf{r}, \Omega)$ is introduced in a purely phenomenological manner, as are rate constants in ordinary chemical kinetics.

The SLE (120) may be used as a general reference point to order the various treatments of the radical pair mechanism described in the literature and to assess their approximate nature.

An intuitive approach for handling the problem stated in eq 120 is to decompose it into the separate problems of spin motion and of diffusion and to assemble their mutual interaction with spin-dependent recombination kinetics in a final step. These aspects will be reviewed separately in the next sections.

\section{Spin Motion}

The various kinds of interactions that have been explicitly treated in the theory of electron spin motion are collected in the following spin Hamiltonian:

$H=\sum_{i=1}^{2}\left(\mu_{\mathrm{B}} \mathbf{B}_{0} \mathbf{g}_{i} \boldsymbol{S}_{i}+\sum_{j} \boldsymbol{I}_{i, j} \boldsymbol{a}_{i, j} \boldsymbol{S}_{i}\right)-J\left(1 / 2+2 \boldsymbol{S}_{1} \boldsymbol{S}_{2}\right)$

The first two terms refer to Zeeman and hyperfine interaction, respectively, where the tensor notation for $\mathbf{g}_{i}$ and $\mathbf{a}_{i, j}$ emphasizes that there are anisotropic contributions to these interactions. The third term denotes the exchange interaction placing the energy of the radical pair triplet by an amount of $2 J$ below the radical pair singlet. (Note, however, that in many applications $J$ is assumed to be negative. For a compilation of other equivalent expressions describing the exchange interaction, cf. ref 57.) Other interactions that are discussed in some contexts are spin-rotational coupling 85,440 and dipolar electron spin-spin interaction. $427,502,505,641$ However, coherent spin motion has not been explicitly calculated with these interactions.

The anisotropic parts of $\mathbf{g}_{i}$ and $\mathbf{a}_{i, j}$ provide couplings to the random motion of the heat bath. Usually they will average out to zero on a time scale short with respect to coherent spin motion; however, their fluctuations cause incoherent relaxation processes, which will be considered below.

Exchange interaction, which is strongly dependent on the radical separation, is also modulated by the radical motion and thus is a time-dependent interaction. With the argument that in freely diffusing radical pairs spin evolution occurs mainly at interradical distances where $J$ may be neglected, the $J$ term is often omitted in explicit treatments of spin motion. Thus we will begin with a survey of the work considering constant Zeeman and hyperfine coupling interactions only.

Having specified the spin Hamiltonian, it is further necessary to define the initial spin state in order to obtain a definite solution for the spin motion. It is customary to use the conditional probabilities ${ }^{\mathrm{T}} p_{\mathrm{S}}(t)$, ${ }^{\mathrm{T}} p_{\mathrm{T}}(t),{ }^{\mathrm{s}} p_{\mathrm{S}}(t)$, and ${ }^{\mathrm{s}_{p_{\mathrm{T}}}(t) \text {. Here the left superscript } \mathrm{T}}$ or $\mathrm{S}$ denotes the multiplicity in which the radical pair was formed at $t=0$ and the right subscript denotes the multiplicity that is probed at time $t$. Due to the reversibility of coherent processes, these will not disturb a 1:3 statistical singlet/triplet mixture of radical pairs. From this principle the following relation between the various probabilities listed can be derived: ${ }^{65,642}$

${ }^{\mathrm{s}} p_{\mathrm{T}}(t)=1-{ }^{\mathrm{s}} p_{\mathrm{S}}(t)=3\left({ }^{\mathrm{T}} p_{\mathrm{S}}(t)\right)=3\left(1-{ }^{\mathrm{T}} p_{\mathrm{T}}(t)\right)$

Radical pairs originating from random encounters of free radicals ( $F$ pairs) correspond to a $1: 3$ statistical mixture of singlet and triplet pairs. Since their first encounter will usually eliminate more of the singlet pairs, the radical pairs being left after this event will behave rather like radical pairs with initial triplet spin.

A more complicated situation concerning the initial spin correlation of radical pairs arises when more than one electron/hole pair is produced in spurs by energyrich particle radiation. Here the total spin of a spur is zero. However, pair contributions within the spur may have singlet or triplet correlation, the probability of which may be obtained from combinatorial analysis. ${ }^{392,395}$ The problem has been somewhat controversial. $^{393,394}$

If the spin Hamiltonian is time independent, it is, in principle, a straightforward matter to determine the spin motion from the eigenvalues $E_{q}$ and eigenfunctions $|q\rangle$ of the spin Hamiltonian ${ }^{643,644}$

$$
\begin{aligned}
{ }^{\mathrm{T}} p_{\mathrm{S}}(t)= & 1 / 3\left\{1-\left[1 / \prod_{i, k}\left(2 I_{1 i}+1\right)\left(2 I_{2 k}+1\right)\right] \times\right. \\
& \left.\sum_{m, m^{\prime}}\left|\sum_{q}\langle\mathrm{~S}, m \mid q\rangle\left\langle q \mid \mathrm{S}, m^{\prime}\right\rangle \exp \left(-i \hbar^{-1} E_{q} t\right)\right|^{2}\right\}
\end{aligned}
$$

The functions $|\mathrm{S}, m\rangle$ denote the radical pair singlet state combined with some definite nuclear spin state:

$$
m=\left(m_{1}, m_{2}, \ldots, m_{i}, \ldots\right)
$$

In eq 123 it is assumed that at $t=0$ each nuclear spin state is represented with equal probability. For radicals with large numbers of nuclear spins, however, the quantum mechanical analysis becomes too extensive to be practically tractable. The largest spin system treated so far in this manner was the radical pair of pyrene and $N, N$-dimethylaniline $\left({ }^{2} \mathrm{Py}^{-}+{ }^{2} \mathrm{DMA}^{+}\right)$where four sets of equivalent magnetic nuclei with altogether 18 nuclear spins were treated numerically ${ }^{643}$ In view of the rapidly increasing numerical effort necessary for treating large spin systems on the basis of eq 123 , it is gratifying to note that a very convenient method of approximate calculation may be applied to the case of many nuclear spins (vide infra).

Furthermore, it has been shown by Schulten ${ }^{65,674}$ that the ensemble-averaged, time-dependent singlet or triplet probability of radical pairs may be represented as the inner product of two spin correlation tensors that may be calculated for each of the radicals separately. 
(a) Exact Solutions of Special Cases. A fairly general case where explicit closed-form expressions have been obtained, a radical pair with two arbitrary nuclear spins $I_{1}$ and $I_{2}$ with one on each radical, has been solved by Salikhov et al. ${ }^{645}$ The solution for the more general case with an arbitrary number of equivalent magnetic nuclei on each radical may be decomposed into contributions of the latter kind. ${ }^{646}$ Corresponding formulas for the case of two magnetically equivalent sets of protons have been given by Brocklehurst ${ }^{647}$ and by Evans and Lawler. ${ }^{648}$ A special example with nine equivalent protons on one radical only has been worked out by Fischer ${ }^{323}$ in the analysis of his experimental results. The model radical pair with one proton on each radical has been considered in detail by Schulten and co-workers. ${ }^{643,649}$ The simplest model radical pair with one nucleus $I=1 / 2$ has been used by many authors.

Whereas the case of general fields becomes rather involved with an increasing number of nuclear spins, the high-field case can be easily handled for an arbitrary number of spins. This simplification is due to the fact that nuclear spin quantum numbers are conserved under high-field conditions since electron-nuclear spin flip-flops are not energy conserving in high magnetic fields. Thus one has to perform only an average over the $\mathrm{S}-\mathrm{T}_{0}$ oscillations for the individual nuclear spin states: 650

$$
{ }^{\mathrm{s}} \mathrm{p}_{\mathrm{T}_{0}}(t)=\left[1 / \prod_{i, k}\left(2 I_{1 i}+1\right)\left(2 I_{2 k}+1\right)\right] \sum_{m} \sin ^{2}\{\omega(m) t\}
$$

with

$$
\omega(m)=1 / 2\left[\left(g_{1}-g_{2}\right) \mu_{\mathrm{B}} B_{0} / \hbar+\sum_{i} a_{1 i} m_{1 i}-\sum_{k} a_{1 k} m_{1 k}\right]
$$

It is evident that the angular frequency expression reflects the rephasing mechanism of electron spin motion.

A very compact expression for the high-field case with $n \times I=1 / 2$ spins only has been derived by Brocklehurst: $: 47$

$$
s_{p_{T_{0}}}=1 / 2\left[1-\cos \left\{t\left(g_{1}-g_{2}\right) \mu_{\mathrm{B}} B_{0} / \hbar\right\} \prod_{k=1}^{n} \cos \left\{a_{k} t / 2\right\}\right]
$$

(b) Approximate Solutions for General Fields. Since the full solution to the problem of spin motion is rather involved in the general case of many nuclear spins and an arbitrary magnetic field, resonable approximations that can be handled by limited numerical effort are desirable. As has been pointed out by Haberkorn ${ }^{331}$ it is often sufficient to know the short-time behavior of spin motion, for which, on the basis of perturbation theory, he was able to derive the following expression:

${ }^{s} p_{\mathrm{T}}(t)=1 / 16\left(\omega_{\mathrm{a}} t\right)^{2}\left[1+2 \sin ^{2}\left(\omega_{\mathrm{L}} t / 2\right) /\left(\omega_{\mathrm{L}} t / 2\right)^{2}\right]$

where $\omega_{\mathrm{L}}$ is the Larmor frequency and $\omega_{\mathrm{a}}$ is an average hyperfine frequency given by

$$
\omega_{\mathrm{a}}=\hbar^{-1}\left[4 / 3 \sum_{i}^{(1)} I_{i}\left(I_{i}+1\right) a_{1, i}{ }^{2}+4 / 3 \sum_{j}^{(2)} I_{j}\left(I_{j}+1\right) a_{2, j}{ }^{2}\right]^{1 / 2}
$$

Equation 126 nicely describes the switching of the spin motion from zero-field to high-field behavior (cf. Figure
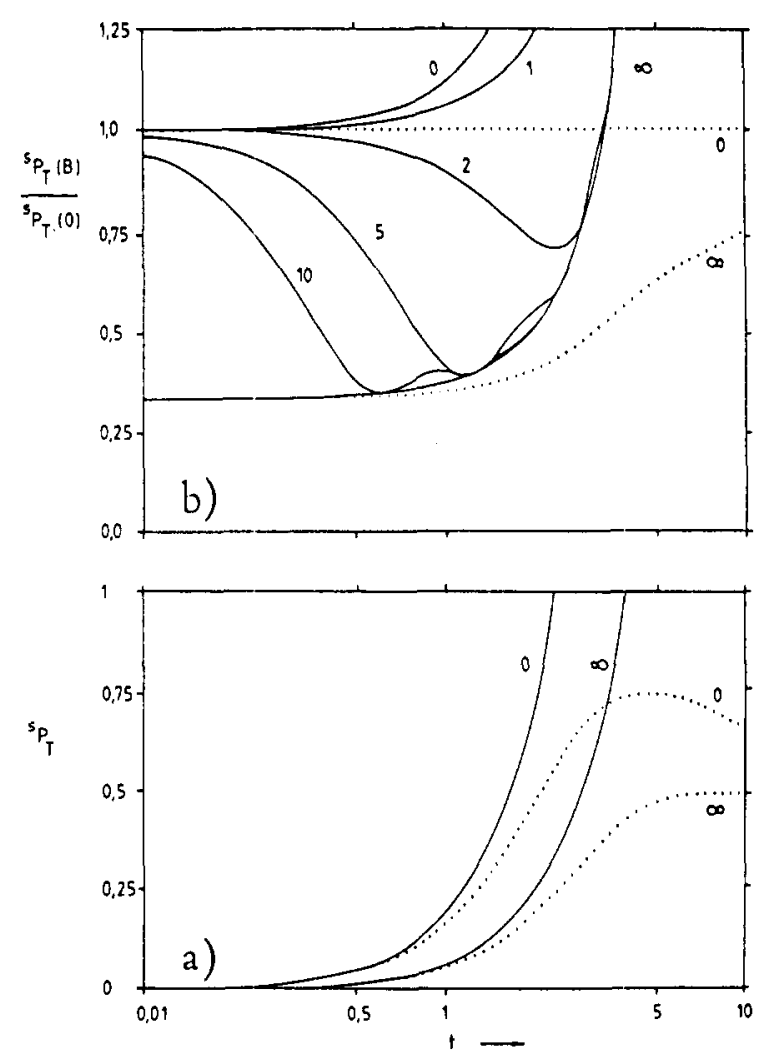

Figure 46. Evolution of triplet character in radical pairs produced with singlet spin $\left({ }^{\mathrm{S}} p_{\mathrm{T}}\right)$. Solid lines: short-time approximation of Haberkorn, 331 eq 126, with $\omega_{\mathrm{a}}=1$ and $\omega_{\mathrm{L}}$ values as indicated in the figure. Dotted lines: zero-field and high-field result corresponding to the semiclassical approximation of Schulten and Wolynes ${ }^{65}$ (eq 129 and 130 with $\tau_{1}=\tau_{2}=4$ ). In diagram $b$ relative values with reference to the semiclassical zero-field value are plotted to demonstrate the intermediate-field cases more clearly. Note that the time scale is logarithmic.

46). The zero-field short-time behavior is described by $3 / 16\left(\omega_{a} t\right)^{2}$, whereas in the high-field case it is $1 / 16\left(\omega_{\mathrm{a}} t\right)^{2}$, indicating that singlet to triplet transitions for two of the triplet substates are quenched in high magnetic fields. The period of time where switching of spin motion from low-field to high-field behavior occurs (cf. also Figure 3 in ref 439) is shifted to earlier times as the field is increased. This reflects the effect of the probing time on $B_{1 / 2}$ observed in several experiments (cf. ref 158, 307, and 312).

A most remarkable method to approximate spin evolution in the general case has been contributed by Schulten and co-workers. ${ }^{65,68,651}$ Here the influence of nuclear spins is approximated by effective classical magnetic fields resulting from a quasi-continuous distribution of nuclear spin orientations. This assumption allows averaging of the nuclear spins, yielding compact expressions for the zero-field and high-field cases and series expansions for the intermediate-field case. The averaged hyperfine contribution of each radical is specified by a characteristic time $\tau_{i}$, given by

$$
\left(\tau_{i}\right)^{-2}=1 / 6 \sum_{k} a_{i k}^{2} I_{i k}\left(I_{i k}+1\right)
$$

The spin-motion expressions for the zero-field and high-field cases, respectively, are given by

$$
\begin{aligned}
& \mathrm{s}_{p_{\mathrm{T}}}\left(t, B_{0}=0\right)= \\
& 3 / 4\left\{1-1 / 9\left[1+2 C\left(t / \tau_{1}\right)\right]\left[1+2 C\left(t / \tau_{2}\right)\right]\right\}
\end{aligned}
$$

with 


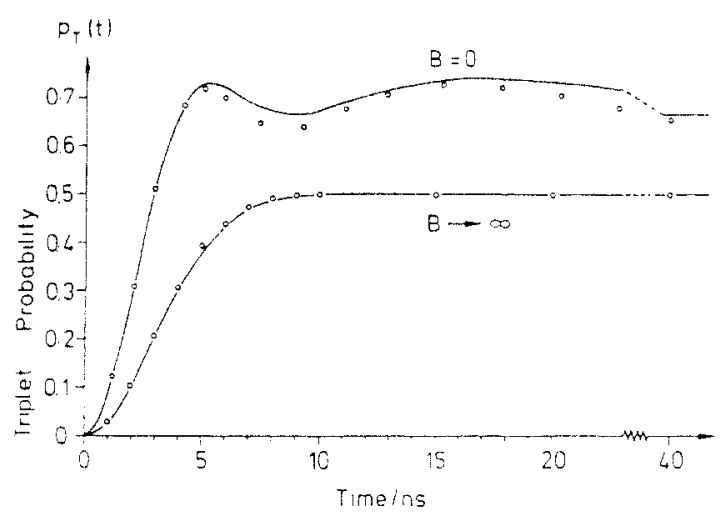

Figure 47. Comparison of the triplet probability of the unpaired spins in a singlet-spin-generated (pyrene) ${ }^{\circ-} /(N, N$-dimethylaniline $)^{*+}$ radical ion pair predicted by the semiclassical theory $y^{65}$ (solid lines) and from an exact quantum mechanical analysis ${ }^{643}$ (data points). Reprinted from ref 65 with kind permission of $K$. Schulten; copyright 1978 American Institute of Physics.

$$
C(x)=\left(1-2 x^{2}\right) \exp \left(-x^{2}\right)
$$

and

$$
\begin{aligned}
& \mathrm{s}_{p_{\mathrm{T}}}\left(t, B_{0} \gg a_{i k}\right)= \\
& 1 / 2\left[1-\exp \left(-t^{2} / \tau_{1}{ }^{2}-t^{2} / \tau_{2}{ }^{2}\right) \cos (\Delta \omega t)\right]
\end{aligned}
$$

with

$$
\Delta \omega=\left(g_{1}-g_{2}\right) \mu_{\mathrm{B}} B_{0} / \hbar
$$

The semiclassical results compare very favorably with exact results for more than three nuclear spins with a sufficient scatter of hyperfine coupling constants (cf. Figure 47).

A modified version of the semiclassical approach, without complete averaging of the hyperfine fields, has been derived by Purtov and Salikhov. ${ }^{652}$ This version is also suitable for calculating CIDNP effects in low magnetic fields. ${ }^{653,654}$

(c) Spin Motion and Exchange Interaction. The exchange interaction is a rapidly decreasing function of radical pair separation, usually expressed in the form

$$
J(r)=J_{0} \exp \left(-\alpha r_{0}\right)
$$

where $J_{0}$ and $\alpha$ are on the order of ${ }^{345}$

$$
\begin{gathered}
J_{0}=-(0.15-0.45) \times 10^{19} \mathrm{rad} / \mathrm{s} \\
\alpha=2.2-2.7 \AA^{-1}
\end{gathered}
$$

In current theories of the radical pair mechanism it has become general usage to neglect the effect of exchange interaction on spin motion during the diffusional trips of the radicals between reencounters. The justification for this is that the main contributions to spin motion are made by diffusive trajectories extending beyond the sphere of influence of exchange interaction. During reencounters the role of this interaction must be treated differently, however, in CIDEP theories on the one hand and CIDNP and MARY theories on the other. Whereas the dwelling time of radical pairs at distances with moderate exchange interaction is essential for producing electron spin polarization, these parts of the radicals' diffusion trajectories are only of minor importance for the total evolution of singlet or triplet character of the pair, which is probed through the reaction probability in a close contact when the exchange energy is high. A rapid increase of exchange interaction in a reencounter has been shown to alter the actual singlet-triplet ratio of the radical pair negligibly. ${ }^{655}$ However, the large exchange interaction during a reencounter destroys the phase relation between singlet and triplet wave functions, which must be taken into account as a new initial condition for the next period of spin evolution in the subsequent diffusion trajectory. ${ }^{646,652,656-658}$ In the early days of the radical pair mechanism, when the debate about the necessity of explicitly taking into account the time dependence of the exchange interaction during separation and encounters of radical pairs was not yet decided, Fischer ${ }^{659}$ proposed a model of adiabatic transitions occurring in the spatial region where the radical pair passes the position with $J=\left\langle E_{\mathrm{hfc}}\right\rangle$ or $\left\langle E_{\text {Zeeman }}\right\rangle$. It was pointed out by Adrian $^{635}$ that this model is not compatible with the uncertainty principle.

Kaptein and Oosterhoff ${ }^{84,631,660}$ treated exchange interaction as a time-independent constituent of the Hamiltonian, comparable with the hyperfine interaction energies. It is easy to incorporate this influence into the equation of spin motion under high-field conditions. Whereas no closed-form expressions have been reported for the general-field case, such a result for a radical pair with one magnetic nucleus of general spin $I$ (hfc constant $a$ ) has been obtained in zero field by Salikov: ${ }^{661}$

$$
\mathrm{s}_{p_{\mathrm{T}}(t)=I(I+1)\left(a^{2} / R^{2}\right) \sin ^{2}(R t / 2)}
$$

with

$$
R^{2}=I(I+1) a^{2}+(2 J+a / 2)^{2}
$$

As a consequence of the factor $a^{2} / R^{2}$ it follows from eq 132 that singlet-triplet transitions are suppressed when the exchange interaction exceeds the strength of hyperfine coupling.

Several authors have studied simple two-level models involving an explicit time dependence of the exchange interaction. Both perturbation ${ }^{662,663}$ and numerical solution methods ${ }^{664}$ have been applied to these problems.

Whereas an explicit treatment of the exchange interaction can be avoided for freely diffusing radical pairs, this is not possible if the radical pairs have only a restricted range of distances available as in chemically linked radical pairs. ${ }^{308,346}$ For such systems a calculation of spin motion has been performed by Schulten and co-workers, ${ }^{347,665}$ applying a computer simulation of the chain-folding dynamics in order to describe the stochastic modulation of exchange interaction on the basis of eq 131. Analyzing their results by means of Kubo's line shape theory, ${ }^{666}$ they could show that the influence of the exchange interaction can be represented by an equivalent static distribution of biradical end-to-end distances. This "equivalent static distribution" is, however, in general not identical with the equilibrium distribution but depends on the dynamics of the motion which is known from many dynamic lineshape phenomena in magnetic resonance.

If radical pairs are confined to diffuse in the interior of micelles, they may also experience an appreciable effective exchange interaction. Evidence for such effects has been provided by CIDNP ${ }^{417}$ and CIDEP ${ }^{445,446}$ experiments, from which, however, largely different orders of magnitude for the "effective" exchange interaction have been extracted. Treatments of spin motion that explicitly take into account the exchange interaction 
under conditions of intramicellar diffusion are so far not available.

(d) Electron Spin Hopping. During the lifetime of an electron-spin pair the hyperfine environment of each electron spin may be changed either by chemical transformations of the radicals, by which so-called consecutive radical pairs are obtained (cf., e.g., ref 667 and 668) or by electron hopping between equivalent molecules, a situation to be considered in systems with high concentrations of electron donors or acceptors. The effect of such hopping processes is to generate a random modulation of the hyperfine interaction. ${ }^{669}$ It has been theoretically investigated for the high-field case of CIDEP and CIDNP by Hore and McLauchlan ${ }^{670}$ and by Salikhov et al. ${ }^{671,672}$ For the case of a radical pair with $1 \times I=1 / 2$ of hfc constant $a$ and electron jumping with a rate constant $k$ between equivalent molecules carrying the magnetic nucleus, the high-field result is ${ }^{732}$

$$
{ }^{\mathrm{s}}{p_{\mathrm{T}_{0}}}(t)=1 / 2\left[1-\cos \left\{\Delta g \mu_{\mathrm{B}} B_{0} t / \hbar\right\} g(t)\right]
$$

with

$$
g(t)=\exp (-k t / 2)[\cosh \{R t\}+(k / 2 R) \sinh \{R t\}]
$$

and

$$
R=\left(k^{2}-a^{2}\right) / 4
$$

In the limit $k \gg a$ eq 133 reduces to

$$
\mathrm{s}_{p_{\mathrm{T}_{0}}(t)=1 / 2\left[1-\exp \left\{-a^{2} t /(4 k)\right\}\right]}
$$

demonstrating that spin evolution is suppressed if hyperfine interactions are averaged over the typical rephasing period.

The general case of electrons hopping between molecules of an arbitrary number of nuclear spins has been tackled with the semiclassical formalism developed by Schulten and co-workers. ${ }^{65,651}$ No analytical expressions have been obtained for the general case but an efficient numerical method is described. It is shown that with increasing hopping rate, the $B_{1 / 2}$ value first increases, as is typical for the general situation of lifetime broadening. However, in the region of high hopping rates, the $B_{1 / 2}$ value decreases toward a limit where the effect of magnetic nuclei on the exchanging molecules averages out to zero. This situation corresponds to the exchange-narrowed limit.

The hopping problem has also been extended to the case of intramolecular electron hopping between two covalently linked donor sites. ${ }^{330,673}$ Here the hyperfine coupling is not averaged to zero in the fast hopping limit but to a finite hyperfine coupling, half as strong as without hopping but with twice the number of nuclear spins.

(e) Paramagnetic Relaxation. The MFD of paramagnetic relaxation times $T_{1}$ and $T_{2}$ was first discussed by Brocklehurst ${ }^{397}$ as a reason for a possible magnetic field sensitivity of radical pair recombination kinetics. Although theoretical magnetic field dependences of $T_{1}$ and $T_{2}$ have been established for various mechanisms such as those involving anisotropic $g$ tensor and hyperfine tensor modulation by molecular tumbling ${ }^{56}$ and spin-rotational relaxation, ${ }^{455}$ no systematic experimental studies of the MFD of $T_{1}$ and $T_{2}$ seem to be available in liquid solutions, because ESR measurements are usually performed at a few fixed frequencies only.
At zero field $T_{1}$ and $T_{2}$ cannot be distinguished. At higher field $T_{1}$ processes cause radical pair spin relaxation between $\left(\mathrm{T}_{+}, \mathrm{T}_{-}\right)$and $\left(\mathrm{T}_{0}, \mathrm{~S}\right)$, whereas $T_{2}$ processes are responsible for $\mathrm{T}_{0} \leftrightarrow \mathrm{S}$ relaxation. Typical values of organic radicals in low-viscosity solvents at room temperature are to be expected in the order of $10^{6} \mathrm{~s}^{-1}$ for both $T_{1}$ and $T_{2}$. (The relaxation times of small linear radicals such as $\mathrm{OH}^{*}$ with unquenched orbital momentum may be considerably shorter. ${ }^{56,400}$ ) Thus, at zero field, paramagnetic relaxation is typically by $2-3$ orders of magnitude slower than coherent spin evolution processes induced by isotropic hyperfine coupling. However, if the external magnetic field exceeds the hyperfine fields, the rate of coherent $\left(\mathrm{T}_{+}, \mathrm{T}_{-}\right) \leftrightarrow\left(\mathrm{T}_{0}\right.$, S) processes will fall below that of the $T_{1}$ process. Then the MFD of $T_{1}$ may be reflected in radical pair recombination kinetics under suitable conditions, e.g., for long cage times in microreactors. ${ }^{427,440}$

The relative contribution of coherent and incoherent spin motion has been described diagrammatically by Brocklehurst $^{647}$ (cf. Figure 48). Here regions B and C are the domains of coherent spin motion. The time evolution of ${ }^{\mathrm{s}} p_{\mathrm{S}}$, depicted as an example, corresponds to a case with a broad distribution of hyperfine coupling constants and may be described by the semiclassical approximation leading to a limiting value for ${ }^{\mathrm{s}} p_{\mathrm{S}}$ of ${ }^{1 / 3}$ in zero field. In cases with a small number of hyperfine-coupled nuclear spins, because of the degeneracy of several stationary states, the limiting value of ${ }^{s} p_{\mathrm{S}}$ may be higher in zero field than in weak fields where the degeneracies are lifted (cf. dotted curve in Figure 48). In high magnetic fields the limiting value of ${ }^{\mathrm{s}} p_{\mathrm{S}}$ is $1 / 2$. At low fields the $T_{1}=T_{2}$ relaxation will establish the statistical singlet character of $1 / 4$ between regions $\mathrm{C}$ and $\mathrm{D}$. In higher fields, however, (Zeeman energy larger than hyperfine energy) the transition from the hyperfine plateau of ${ }^{\mathrm{s}} p_{\mathrm{S}}=0.5$ to the statistical limit of $\mathrm{s}_{p_{\mathrm{S}}}=0.25$ will be due to the $T_{1}$ process between regions $\mathrm{D}$ and $\mathrm{E}$, the exact position on the time scale depending on the strength of the magnetic field. An explicit analytical expression for ${ }^{\mathrm{s}} p_{\mathrm{S}}(t)$ has been derived for the high-field case by Klein and Voltz ${ }^{388}$ and by Kubarev et al.:294

$$
\begin{aligned}
& { }^{\mathrm{s}} p_{\mathrm{S}}(t)= \\
& \mathrm{L}_{2}\left(\prod_{i, j}\left(2 I_{i}+1\right)\left(2 I_{j}+1\right)\right)^{-1}\left[\sum_{m}\left\{\cos \left(\omega_{m} t\right) \exp \left(-2 \Gamma_{2} t\right)\right\}+\right. \\
& \left.\left\{1+\exp \left(-2 \Gamma_{1} t\right)\right\} / 4\right]
\end{aligned}
$$

with $\omega_{m}$ as defined in eq 124a.

Equation 135 was derived from a Redfield type equation of motion of the spin density matrix, wherein it was assumed that $T_{1}$ and $T_{2}$ are independent of the nuclear spin states and do not involve nuclear spin flips. Klein and Voltz ${ }^{388}$ used $T_{1}$ only, setting

$$
\Gamma_{1}=\Gamma_{2} / 2=1 /\left(2 T_{1}\right)
$$

whereas Kubarev et al. ${ }^{294}$ used

$$
\begin{aligned}
& \Gamma_{1}=1 /\left(2 T_{1}\right) \\
& \Gamma_{2}=1 /\left(2 T_{2}\right)
\end{aligned}
$$

From an ad hoc argument Fischer ${ }^{323}$ arrived at an analogous expression as to eq 135 . He used eq 137 together with the assumption $T_{1}=T_{2}$, which is justified in the case of spin-rotational relaxation as the dominant mechanism. 


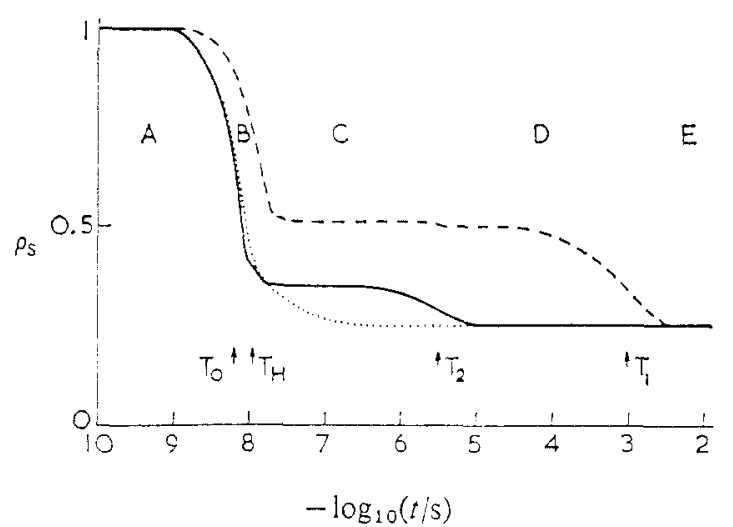

Figure 48. Schematic representation of radical pair spin evolution $p_{\mathrm{S}}(t)$ (singlet character after generation with singlet spin) in various time regimes. Solid line, zero field: dotted line, very low field: dashed line, high field. For details, cf. text. Reprinted with permission from ref 647; copyright 1976 the Royal Society of Chemistry.

Hayashi and Nagakura ${ }^{427}$ used a purely reaction-kinetic approach to incorporate coherent and relaxation contributions of spin motion into a treatment of intramicellar radical pair recombination. Their contribution is particularly remarkable insofar as they were the first to attempt a quantitative account of the MFD of spin relaxation and its relation to the MFD of intramicellar radical pair recombination.

\section{The Dynamic Probability Function}

If it is assumed that spin motion and diffusion can be separated, which is strictly justified only if recombination to products of different multiplicity occurs with the same rate constant, ${ }^{649}$ the radical pair recombination rate $(\partial[\mathrm{RP}] / \partial t)_{\mathrm{S}(\mathrm{T})}$ into singlet (triplet) reaction channels may be expressed as

$$
(\partial[\mathrm{RP}] / \partial t)_{\mathrm{S}(\mathrm{T})}=-P_{\mathrm{S}(\mathrm{T})}(t) f_{\mathrm{S}(\mathrm{T})}(t)[\mathrm{RP}]_{0}
$$

The function $f_{\mathrm{S}(\mathrm{T})}(t)$ may be conceived as a pure-spin recombination rate "constant" of radical pairs. (The normal case is that $f_{\mathrm{S}}(t) \neq 0$ and $f_{\mathrm{T}}(t)=0$. With reference to this situation we shall omit the multiplicity subscript in the following.) The function $f(t)$ has been termed the "dynamic probability factor" by Deutch. ${ }^{457}$ It will be determined mainly by the distribution of interradical distances together with the distance dependence of the recombination rate constant, and it accounts for the combined influence of $\Gamma(\mathbf{r}, \mathbf{Q})$ and $\boldsymbol{K}(\mathbf{r}, \mathbf{Q})$ in eq 120. Thus $f(t)$ will essentially depend on the diffusive motion of the radical pair and describe the statistics of reencounters. On the other hand, $f(t)$ will be also used to include the disappearance of radical pairs by competing reaction channels. Of course, the usefulness of eq 138 depends on the degree of independence of the functions $p_{\mathrm{S}(\mathrm{T})}(t)$ and $f_{\mathrm{S}(\mathrm{T})}(t)$. Several mathematical forms of the function $f(t)$ have been critically examined by Salikhov et al..$^{52}$ form

(a) The Exponential Model. Using the exponential

$$
f(t)=k_{\mathrm{s}} \exp \left(-k_{0} t\right)
$$

as was customary in the early versions of the radical pair mechanism, ${ }^{84,94,631,675,676}$ is probably the crudest and least realistic assumption. For neutral radical pairs in homogeneous solution it yields only a pure description of the reencounter statistics. However, it should be noted that for radical pairs in microreactors like micelles ${ }^{677,678}$ or microemulsion nanodroplets ${ }^{441}$ it is probably a good approximation for the reencounter statistics. In homogenous solutions it might be appropriate under conditions of strong scavenging. ${ }^{92}$ In the case of solidstate-like environments, e.g., in photosynthetic reaction centers, the pure-spin reaction rate constant will also decrease with radical separation in an exponential fashion. However, under conditions where spin motion and reaction occur in the same radical pair conformation, eq 138 is usually a bad approximation and a compact solution of the SLE is preferable (vide infra).

A considerable improvement within the exponential model can be achieved by using a two-site radical pair to simulate the reencounter statistics:

$$
(\mathrm{RP})_{1} \rightleftharpoons(\mathrm{RP})_{2} \rightarrow \text { escape }
$$

Here $f(t)$ is given by the fraction of radical pairs $(\mathrm{RP})_{1}$ present in site 1 . Use of this model has been made by Purtov and Salikhov ${ }^{680}$ in order to account for effects of molecular rotation on the recombination probability. The two-site model is more widely used in methods involving direct solution of the stochastic Liouville equation (vide infra).

(b) The Noyes Function of First Reencounter. A most important step forward was made by Adrian's ${ }^{637}$ introduction of the Noyes reencounter concept ${ }^{638}$ into radical pair theory. According to this, $f(t)$ in eq 138 describes the time distribution of first reencounters, i.e., the secondary solvent cage effect, which allows for much longer periods of spin evolution than the primary cage lifetime. Adrian used the expression

$$
\begin{gathered}
f(t)=0, \quad t<2 \tau \\
f(t)=0.24 /\left[\tau(t / \tau+0.44)^{3 / 2}\right], \quad t \geq 2 \tau
\end{gathered}
$$

which was adapted from the Noyes random flight result for the case where single-step diffusive displacements $d$ equal the radical encounter diameter $a$ and where the discrete number of diffusive steps $N$ has been replaced by the continuous variable $t / \tau$. The inverse of the jump frequency, the hopping time constant $\tau$, was assumed to be $10^{-11}-10^{-12} \mathrm{~s}$.

Kaptein ${ }^{660,681}$ used another expression suggested by Noyes for general $a / d$

$$
f(t)=m t^{-3 / 2} \exp \left(-\pi m^{2} / p^{2} t\right)
$$

where $p$ and $m$ are defined by

$$
\begin{gathered}
p=\int_{0}^{\infty} f(t) \mathrm{d} t \approx 1-(1 / 2+3 a / 2 d)^{-1} \\
m=1.036(1-p)^{2}(a / d)^{2} \tau^{1 / 2}
\end{gathered}
$$

Kaptein used $m=10^{-6}$ and $\tau=10^{-11} \mathrm{~s}$.

It is common to both eq 141 and 142 that $f(t)$ decreases as $t^{-3 / 2}$ at long times. As long as the spin motion is slow on a time scale measured in units of $\tau$, the exact form of $f(t)$ at short times is irrelevant.

Haberkorn ${ }^{682}$ pointed out that eq 141 may be used as a good approximation even if $d<a$. However, in this case $\tau$ should be given the value of $a^{2} / D$, where $D$ is the sum of the radical diffusion coefficients.

(c) Continuous-Diffusion Models. Continuousdiffusion models were first applied to geminate radical 
pair recombination by Deutch ${ }^{437}$ and by Abell and Mozumder. ${ }^{683}$ In these treatments $f(t)$ was obtained as the diffusive flux into an absorbing boundary at $r=a$ (Smoluchowski boundary condition) for an initial $\delta$ distribution at $r=r_{0}$. For neutral radical pairs the result for $f(t)$ is ${ }^{457}$

$f(t)=\left[D a\left(r_{0}-a\right) / 2 \pi^{1 / 2} r_{0}\left(D t^{3 / 2}\right)\right] \exp \left[-\left(r_{0}-a\right)^{2} / 4 D t\right]$

For radical ion pairs no closed-form expression can be obtained. The interpretation of the result given by eq 145 as the first-arrival time distribution has been criticized by Haberkorn ${ }^{682}$ and by Razi Naqvi et al. ${ }^{684}$

Schulten and Schulten ${ }^{649}$ determined the function $f(t)$ by solving the Smoluchowski equation with boundary conditions corresponding to the production of the radical pair at the encounter distance $a$ and to the radiation boundary condition

$$
D\left(\frac{\partial W}{\partial r}\right)_{r=a}=-\kappa W(a)
$$

where $W(r)$ is the probability density that the radical pair distance is $r$. The parameter $\kappa$ has the dimension of a linear velocity and determines the total pure-spin reaction probability $\Phi_{0}$ of the radical pair

$$
\Phi_{0}=\kappa /(\kappa+D / a) \equiv \kappa / \alpha
$$

The result for $f(t)$ is

$$
\begin{aligned}
f(t)= & \kappa /(\pi D t)^{1 / 2}[1- \\
& \left.\alpha(\pi t / D)^{1 / 2} \exp \left(\alpha^{2} t / D\right) \operatorname{erfc}\left(\alpha(t / D)^{1 / 2}\right)\right]
\end{aligned}
$$

For radical ion pairs the problem of geminate recombination was investigated by numerical integration of the corresponding Smoluchowski equation. ${ }^{643,649}$

Razi Naqvi et al. ${ }^{684}$ have compared the model of first reencounter with the diffusional model of radical pair recombination. According to their analysis, eq 148 with $\kappa=d \nu / 4$ ( $\nu$ is the jump frequency in the random flight model) yields the correct expression for the first "arrival probability" whereas expression 145 is an approximation that may lead to serious errors if the short-time limit is of any importance.

Tarasov et al. ${ }^{559}$ subjected the diffusional model to the boundary conditions of microscopic spherical reactors. They determined the coefficients of a series expansion for the reencounter probability function $f(t)$.

\section{Assembling Spin Motion, Diffusion, and Recombination}

So far, most theoretical work on the radical pair mechanism has been concerned with time-integrated yields $Y$ of radical pair recombination. In many cases the function $f(t)$ describing diffusion and pure-spin recombination is combined with spin motion, as described by $p_{\mathrm{S}}(t)$ or $p_{\mathrm{T}}(t)$, according to

$$
Y_{\mathrm{S}(\mathrm{T})}=\int_{0}^{\infty} p_{\mathrm{S}(\mathrm{T})}(t) f(t) \mathrm{d} t
$$

A number of representative references, wherein methods for assembling various combinations of different types of spin motion and dynamic probability factors $f(t)$ have been provided, are compiled in Tahle 18. There are also included references to other work, where more advanced methods combining independent treatments of spin motion and diffusion are applied.

(a) Exponential Radical Pair Decay. The typical expression appearing when integrating coherent spin motion with exponential radical pair decay is

$$
(1 / \tau) \int_{0}^{\infty}[\sin (\text { nat })]^{2} \exp (-t / \tau) \mathrm{d} t=\frac{2 n^{2} a^{2} \tau^{2}}{1+4 n^{2} a^{2} \tau^{2}}
$$

Applying, e.g., the spin motion expression for a radical pair with one nuclear $\operatorname{spin}(I=1 / 2)$, the result for the integral (149) is ${ }^{10,52}$

$$
\begin{aligned}
\mathrm{s}_{Y_{\mathrm{S}}}\left(B_{0}=0\right)=1-3 / 8(a \tau)^{2}\left[1+(a \tau)^{2}\right]^{-1} \\
{ }^{\mathrm{s}} Y_{\mathrm{S}}\left(B_{0} \rightarrow \infty\right)=1-1 / 2(a \tau)^{2}\left[4+(a \tau)^{2}\right]^{-1}
\end{aligned}
$$

For $a \tau \ll 1$ eq $151 \mathrm{a}, \mathrm{b}$ exhibit a square dependence of the recombination yield on the hyperfine coupling constant. Furthermore, under this condition, the $\mathrm{S} / \mathrm{T}$ conversion effect on $Y$ is smaller in a strong magnetic field than at zero field. The latter feature is reversed, however, if $a \tau>1$. This is a peculiarity of radical pairs with few nuclear spins, all localized on one radical. The phenomenon has been analyzed in some detail by Sarvarov and Salikhov. ${ }^{687}$ The anomaly may be attributed to the $\mathrm{S} / \mathrm{T}$ oscillation amplitude, which is increased by a magnetic field only in the special case of few nuclear spins on one radical. The oscillation frequency behaves uniformly for all hyperfine coupling cases: It is slowed down in a magnetic field (cf. eq 126).

(b) Diffusional Treatments of Reencounters. Using the Noyes reencounter function $f(t)$ (eq 142), one obtains by integrating with coherent spin motion terms $^{52}$

$$
Y=\int_{0}^{\infty} \mathrm{d} t f(t) \sin ^{2}(\omega t)=p / 2[1-\cos (c) \exp (-c)]
$$

with

$$
c=(2 m / p)(\pi \omega)^{1 / 2}
$$

Expansion to lowest order in $c$ yields for large $p / m$

$$
Y(c \rightarrow 0)=c(p / 2)=m(\pi \omega)^{1 / 2}
$$

This expression with its characteristic square root dependence on the singlet-triplet oscillation frequency is often used in work referring to the radical pair reencounter model.

An exponential factor may appear in the integrand of eq 152, either due to consideration of scavenging processes on the reencounter probability function or by consideration of relaxation processes in the spin motion. Corresponding results with $f=m t^{-3 / 2}$ have been reported by Buchachenko and Makarian, ${ }^{679}$ and with the full Noyes expression (eq 142) by Fischer ${ }^{323}$ or, using the equivalent Deutch formula, by Kubarev et al. ${ }^{294}$ The most general analytical result for an integral of the type of eq 152 has been obtained by Beljakov and $\mathrm{Bu}$ chachenko ${ }^{694}$ with the Razi Naqvi reencounter expression modified by a scavenging exponential. The series expansion coefficients obtained by Tarasov et al.$^{559}$ for $f(t)$ in micellar microreactors have been used to obtain corresponding expansion coefficients for integrals of the type of eq 152.

Atkins $^{689}$ cast the combined problem of solving the diffusional equation for reencounters and performing 
TABLE 18. Theory of the Radical Pair Mechanism: Assembling Spin Motion, Diffusion, and Recombination

\begin{tabular}{|c|c|c|c|c|c|c|c|}
\hline year & ref & $\begin{array}{l}\exp \\
f(t)\end{array}$ & $\begin{array}{c}\text { Noyes } \\
f(t)\end{array}$ & $\begin{array}{c}\text { cont-diff } \\
f(t)\end{array}$ & $\begin{array}{c}\text { mutli } \\
\text { reencounter }\end{array}$ & $\begin{array}{l}\text { spin-motion } \\
\text { equiv rate } \\
\text { constants }\end{array}$ & $\begin{array}{c}\mathrm{T} / \mathrm{S} \text {-coupled } \\
\text { diff eq }\end{array}$ \\
\hline 1969 & Kaptein and Oosterhoff ${ }^{84,631}$ & $x$ & & & & & \\
\hline 1970 & Closs et al.634,675 & $x$ & & & & & \\
\hline $1970 / 1971$ & Adrian $^{635,636}$ & & $x$ & & & & \\
\hline \multirow[t]{2}{*}{1971} & Lawler and Evans ${ }^{94}$ & $x$ & & & & & \\
\hline & Tomkievicz and Cocivera ${ }^{685}$ & $x$ & & & & $x$ & \\
\hline \multirow[t]{2}{*}{1972} & Sagdeev et al. 639 & & $\times$ & & & & \\
\hline & Kaptein 660 & & $x$ & & $x$ & & \\
\hline 1973 & Atkins $^{676}$ & $x$ & & & & & \\
\hline $1973 / 1974$ & Buchachenko and Markarian ${ }^{679,686}$ & $x$ & $x$ & & & & \\
\hline 1975 & Sarvarov and Salikhov ${ }^{687}$ & $x^{a}$ & $x$ & & & & \\
\hline \multirow[t]{2}{*}{1976} & Haberkorn and Michel-Beyerle ${ }^{688}$ & & $\hat{x}$ & & & & \\
\hline & Schulten et al. . $^{302}$ & $x^{6}$ & & & & & $x^{b}$ \\
\hline \multirow[t]{7}{*}{1977} & Atkins 689 & & & $x$ & & & \\
\hline & Haberkorn ${ }^{682}$ & & $x$ & & $x$ & & \\
\hline & Haberkorn $^{331}$ & & & $x$ & & & \\
\hline & Schulten and Schulten ${ }^{649}$ & & & $x$ & & & \\
\hline & Werner et al. ${ }^{643}$ & & & $\hat{x}$ & & & $x^{c}$ \\
\hline & Pedersen $^{657}$ & & & & $x^{d}$ & & \\
\hline & Salikhov ${ }^{690}$ & & & & $x^{d, e}$ & & \\
\hline \multirow[t]{2}{*}{1978} & Hayashi and Nagakura ${ }^{325}$ & & $x$ & & & & \\
\hline & Bube et al. ${ }^{112}$ & & & & & $x$ & \\
\hline \multirow[t]{4}{*}{1979} & Purtov and Salikhov ${ }^{680}$ & & & & $x^{e}$ & & \\
\hline & Kubarev et al. ${ }^{294}$ & & & $x$ & & & \\
\hline & Knapp and Schulten ${ }^{651}$ & & & $x$ & & & \\
\hline & Schulten and Epstein 68 & & & $\hat{x}$ & $x_{f}^{f}$ & & \\
\hline \multirow[t]{2}{*}{1980} & Purtov and Salikhov 652,658 & & $x$ & & $x^{d}$ & & \\
\hline & Sterna et al. ${ }^{577}$ & & & & & $x^{g}$ & $x^{g}$ \\
\hline 1981 & Tarasov et al. .559 & & & $x^{h}$ & & & \\
\hline $1982 / 1984$ & Brocklehurst $^{691-693}$ & & & $\hat{x}$ & $x^{i}$ & & \\
\hline \multirow[t]{3}{*}{1983} & Salikhov ${ }^{646}$ & & & & $x^{d}$ & & \\
\hline & Belyakov and Buchachenko ${ }^{694}$ & & & $x$ & & & \\
\hline & Fischer 323 & & $x$ & & & & \\
\hline \multirow[t]{2}{*}{1984} & Hayashi and Nagakura ${ }^{427}$ & & & & & $x$ & \\
\hline & Knapp and Lersch ${ }^{673}$ & & $x^{j}$ & & & & \\
\hline $1985 / 1986$ & Bittl and Schulten, ${ }^{347}$ Bittl et al. ${ }^{665}$ & $x^{k}$ & & & & & \\
\hline 1986 & Schulten and Bittl ${ }^{695}$ & $x^{k}$ & & & & & \\
\hline 1987 & Baumann et al. ${ }^{441}$ & $x$ & & & $x^{d}$ & & \\
\hline
\end{tabular}

${ }^{a}$ Here also the case $f(t)=1$ is considered, yielding the long-time average of $p_{\mathrm{S}}(t)$. ${ }^{b}$ Radial pair dissociation as first-order rate process in coupled $\mathrm{T} / \mathrm{S}$ rate equations. ${ }^{c}$ Coupled diffusion equations for ${ }^{1,3} \mathrm{RPs}$ with exact spin motion. ${ }^{d}$ Summation of operator series. ${ }^{e} \mathrm{C}$ Combined with two-site radical pair model. ${ }^{f}$ Reencounters summed by Monte Carlo path integral calculation. ${ }^{8}$ Coupled diffusion equations for ${ }^{1,3} \mathrm{RPs}$ with $\mathrm{T} \rightarrow \mathrm{S}$ transitions described by rate constants. ${ }^{h}$ Boundary conditions corresponding to micellar solubilization of radical pair. ${ }^{i}$ Monte Carlo averaging of reencounters based on eq 145. if $\propto t^{-3 / 2}$ averaging of spin correlation including two-site electron hopping. ${ }^{k}$ Applying biradical spin motion, modulated by stochastic variation of exchange interaction (Monte Carlo calculation).

time integration into a compact mathematical form, where the recombination probability is obtained directly as the Laplace transform of a suitable Green's function of the diffusion equation.

Several authors have used numerical methods to integrate eq 138. This turned out to be necessary in cases where diffusion was treated in a Coulombic potential $^{643,649}$ or where spin motion was too complex, as in systems with many magnetic nuclei ${ }^{643}$ or with electron hopping. ${ }^{651}$

Integration of eq 138 has also been performed with a variable upper time limit, using the simplified expression of eq $148 .^{649}$ Applying the short-time expansion of spin motion (eq 126), Haberkorn ${ }^{331}$ obtained

$$
\begin{array}{r}
\mathrm{s}_{Y_{\mathrm{T}}}(t)=\left(\lambda / 2 \pi^{1 / 2}\right) \int_{0}^{t}\left(\tau_{\mathrm{D}} / t^{\prime 3}\right)^{1 / 2} \times \\
\mathrm{s}_{p_{\mathrm{T}}}\left(t^{\prime}\right) \mathrm{d} t=\left(\lambda / 48 \pi^{1 / 2}\right)\left(\omega_{\mathrm{a}} \tau_{\mathrm{D}}\right)^{1 / 2}\left(\omega_{\mathrm{a}} t\right)^{3 / 2}\{1+ \\
(48 / \pi) S\left[\left(2 \omega_{\mathrm{L}} t / \pi\right)^{1 / 2}\right] /\left(2 \omega_{\mathrm{L}} t / \pi\right)^{3 / 2}- \\
\left.6 \sin ^{2}\left(\omega_{\mathrm{L}} t / 2\right) /\left(\omega_{\mathrm{L}} t / 2\right)^{2}\right\}
\end{array}
$$

where $\lambda$ is the spin-allowed reaction probability per encounter and $S[x]$ is a Fresnel integral. (c) Summing Up Contributions of Repeated Reencounters. Contributions of repeated reencounters to the total recombination yield of the radical pair will become more important the higher the first reencounter probability $p$ and the slower the spin motion. Kaptein $^{660}$ was the first to consider repeated reencounters in the case of original triplet radical pairs whereby each reactionless reencounter was treated as a situation equivalent to the initial production of the triplet pair. Thus the total recombination yield is just the first reencounter yield times $1 /(1-p)$, which is the sum of an infinite geometric series.

The formalism accounting for the effect of spin motion in repeated reencounters was greatly improved in contributions by Pedersen ${ }^{67}$ and by Salikhov. ${ }^{690}$ Spin evolution between two reencounters is described by a time-evolution superoperator $\boldsymbol{M}$ which is time averaged over the distribution of first reencounters $f(t)$. The total recombination yield summed over all reencounters where singlet radical pairs react with a probability of $\lambda$ may be written and evaluated as follows:

$$
\begin{aligned}
Y_{\mathrm{S}}=\operatorname{Tr}\left\{\boldsymbol{Q}_{\mathbf{S}} \lambda \sum_{n=0}^{\infty}\left[\boldsymbol{M}\left(\boldsymbol{I}-\lambda \boldsymbol{Q}_{\mathrm{S}}\right)\right]^{n} \rho(0)\right\}= \\
\operatorname{Tr}\left\{Q_{\mathrm{S}} \lambda\left[\boldsymbol{I}-\boldsymbol{M}\left(\boldsymbol{I}-\lambda \boldsymbol{Q}_{\mathrm{S}}\right)\right]^{-1} \rho(0)\right\}
\end{aligned}
$$


Here $\rho(0)$ is the spin density matrix at the instant of radical pair production, and the superoperator $\boldsymbol{Q}_{\mathrm{S}}$ is defined as

$$
\mathbf{Q}_{\mathrm{S}} \rho=\mathbf{Q}_{\mathrm{S} \rho} \mathbf{Q}_{\mathbf{S}}
$$

where $Q_{\mathrm{S}}$ is the normal projection operator onto the radical pair singlet state. $I$ is the identity operator.

Using the form of eq 145 for $f(t)$ and considering only coherent $T_{0}-S$ transitions, Pedersen ${ }^{657}$ derived closedform expressions for $Y_{S}$ under different initial conditions ( $\mathrm{T}_{0}, \mathrm{~S}$, or $\mathrm{F}$ pairs). Furthermore, he could show that, independent of the particular form of $f(t)$, the results can be expressed in terms of two basic quantities, $\Lambda$ and $F^{*}$, only. These are defined as

$$
\Lambda={ }^{\mathrm{S}} Y_{\mathrm{S}}(\omega=0)=\lambda /(1-p+p \lambda)
$$

where $p$ is the probability of at least one reencounter (cf. eq 143), and

$$
F^{*}(\omega)={ }^{\top} Y_{S}(\lambda=1, \omega)
$$

Here $\omega$ is the frequency of $\mathrm{T}_{0}-\mathrm{S}$ transitions.

The quantities $\Lambda$ and $F^{*}$ have the meaning of a total recombination probability of singlet pairs if spin evolution is excluded $(\Lambda)$ and the total recombination probability of initial $T_{0}$ radical pairs if every singlet reencounter leads to recombination $\left(F^{*}\right)$. With these quantities the following relations hold, independent of the particular diffusion model applied to describe reencounters:

$$
\begin{aligned}
& \mathrm{T}_{0} Y_{\mathrm{S}}(\lambda, \omega)=\Lambda F^{*} /\left[1+F^{*}(1-\Lambda)\right] \\
& \mathrm{s}_{\mathrm{S}}(\lambda, \omega)=\Lambda\left\{1-F^{*}(1-\Lambda) /\left[1+F^{*}(1-\Lambda)\right]\right\} \\
& { }^{\mathrm{F}} Y_{\mathrm{S}}(\lambda, \omega)=(\Lambda / 2)\left\{1+\Lambda F^{*} /\left[1+F^{*}(1-\Lambda)\right]\right\}
\end{aligned}
$$

Setting

$$
\mathrm{T}_{0} Y_{\mathrm{S}}(\lambda, \omega)=3\left({ }^{\mathrm{T}} Y_{\mathrm{S}}(\lambda, \omega)\right)
$$

one can derive

$$
{ }^{\mathrm{s}} Y_{\mathrm{S}}(\lambda, \omega)=\Lambda-3(1-\Lambda)\left({ }^{\mathrm{T}} Y_{\mathrm{S}}(\lambda, \omega)\right)
$$

a general relation between total singlet recombination yield of initial singlet and triplet radical pairs that has been obtained by Salikhov ${ }^{642}$ on the basis of a kinetic interpretation of thermodynamic equilibrium.

The reencounter operator formalism has been extensively applied by Salikhov and co-workers, ${ }^{680}$ who obtained closed-form expressions for the recombination yields of singlet, triplet, and $F$ pairs, considering the combined influence of anisotropic reactivity and rotational diffusion, ${ }^{690}$ using semiclassical models of spin motion $^{652}$ and model radical pairs where spin motion may be conveniently described in zero field ${ }^{646}$ and general fields. ${ }^{658}$

Schulten and Epstein ${ }^{68}$ considered radical pair reencounters in the context of a path-integral method with numerical Monte Carlo simulation of radical pair diffusion trajectories. Baumann et al. ${ }^{441}$ applied the multireencounter formalism using an exponential reencounter probability function for radical pairs in water nanodroplets in w/o microemulsions.

(d) Use of Spin-Motion-Equivalent Rate Constants and Master Equations. It would appear convenient from the chemist's point of view to approximate the stochastic Liouville equation for the spin density matrix by coupled rate equations for the concentrations of singlet and triplet radical pairs. The first step toward this end would be to describe the radical pair singlettriplet conversion by first-order rate constants $k_{\mathrm{TS}}$ and $k_{\mathrm{ST}}$, respectively. Of course, for coherent processes this must be a rather crude approximation, but it will be appropriate for the relaxational type of spin motion, as has been applied, e.g., by Hayashi and Nagakura ${ }^{427}$ to the problem of intramicellar radical pair recombination kinetics. There have been also attempts to correlate coherent spin motions with rate constants, using the following expression establishing a suitable correlation with the reencounter model:

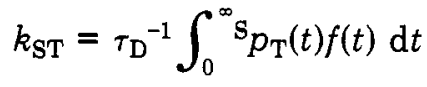

This type of relation has been used by Tomkiewicz and Cocivera ${ }^{685}$ applying the exponential form of $f(t)$ and by Bube et al. ${ }^{112}$ with the Noyes reencounter function (eq 141, using $\tau=\tau_{\mathrm{D}}=a^{2} / D$ ).

Actually, spin motion can be strictly separated from diffusion and recombination only if triplet and singlet radical pairs have identical recombination probabilities. Spatial inhomogeneities in singlet and triplet pair distribution arise, however, at the reaction zone when recombination rates are spin dependent. The reencounter model represents a convenient basis for taking different reactivities of singlet and triplet radical pairs into account. The spin motion on each diffusional trajectory between two reencounters begins with a spin situation originating from the last encounter. A suitable way to incorporate this into the formalism is the operator power series outlined above. However, whereas this method can easily provide the overall recombination yield, it cannot adequately describe the explicit time dependence of the recombination yield, since at a given instant of time there are simultaneous contributions of different orders of reencounters.

Without abandoning the treatment of spin motion as an independent process, the real-time recombination kinetics for different reactivities of singlet and triplet radical pairs has been described by coupled rate equations in several papers by Schulten and co-workers. ${ }^{302,643,649}$ Within the exponential model of dissociation these rate equations are as follows:

$$
\begin{gathered}
\dot{c}_{\mathrm{S}}=-\left(c_{\mathrm{S}}+c_{\mathrm{T}}\right)\left({ }_{\mathrm{S}} \dot{p}_{\mathrm{T}}\right)-\left(k_{\mathrm{S}}+k_{\mathrm{esc}}\right) c_{\mathrm{S}} \\
\dot{c}_{\mathrm{T}}=\left(c_{\mathrm{S}}+c_{\mathrm{T}}\right)\left(\mathrm{S}_{\dot{p}_{\mathrm{T}}}\right)-\left(k_{\mathrm{T}}+k_{\mathrm{esc}}\right) c_{\mathrm{T}}
\end{gathered}
$$

Here $c_{\mathrm{S}}(t)$ and $c_{\mathrm{T}}(t)$ are the concentrations of singlet and triplet radical pairs, respectively. The quantity ${ }_{\dot{p}_{\mathrm{T}}}$, the time derivative of ${ }^{s} p_{\mathrm{T}}$ obtained from separately solving the spin evolution problem, is an effective rate "constant" of $\mathrm{S} / \mathrm{T}$ transitions. The approximation inherent in eq 163a,b is the assumption that the rate of spin evolution should be in effect independent of the $c_{\mathrm{S}} / c_{\mathrm{T}}$ ratio although this will deviate from the $p_{\mathrm{S}} / p_{\mathrm{T}}$ ratio of pure spin motion if $k_{\mathrm{S}}$ and $k_{\mathrm{T}}$ are different.

The concept underlying eq 163a,b has been also applied with the continuous diffusion model, whereby the equations turn into partial differential equations for $c_{\mathrm{S}}(t, r)$ and $c_{\mathrm{T}}(t, r)$. The rate constant $k_{\text {esc }}$ is replaced by a diffusion operator with suitable boundary conditions, and $k_{\mathrm{S}}$ and $k_{\mathrm{T}}$ become $r$-dependent "optical potentials". ${ }^{643}$ The solutions of equations 163a,b and 
their continuous-diffusion counterparts have been obtained by numerical integration. The results for the diffusional model compare favorably with those obtained from numerical integration of the full SLE. ${ }^{643}$

Coupled diffusional equations for $c_{\mathrm{S}}$ and $c_{\mathrm{T}}$ have also been applied by Sterna et al. ${ }^{577}$ to model the magnetic isotope effect at zero field, however with the cruder approximation of actually time-independent rate constants for singlet-triplet conversions. The boundary conditions considered by these authors were chosen so as to apply for geminate recombination in homogeneous solutions and in micelles.

\section{Treatments Based on Direct Solutions of Stochastic Liouville Equations}

The necessity of treating spin motion and recombination together arises when singlet and triplet pairs recombine with different rate constants. As has been analyzed in some detail by Schulten et al. ${ }^{302}$ and by Haberkorn and Michel-Beyerle, ${ }^{533}$ singlet-triplet transitions are slowed down if singlet and triplet levels of the radical pair are differently broadened by spin-selective rate processes. The appropriate method for a combined treatment of spin motion and spin-dependent reaction kinetics is to use statistical Liouville equations.

Density matrix equations of motion have long been used to describe combined coherent and incoherent processes in magnetic resonance. ${ }^{696} 698$ Thus it was only necessary also to incorporate the kinetic effect of dissociation and spin-selective recombination of paramagnetic particle pairs. Two forms of the spin-selective recombination term have been suggested. For selective singlet pair recombination they read as follows:

$$
\begin{gathered}
\boldsymbol{K}_{\mathrm{S} \rho}=-k_{\mathrm{S}} / 2\left[Q_{\mathrm{S}}, \rho\right]_{+}=-k_{\mathrm{S}} / 2\left(Q_{\mathrm{S}} \rho^{+} \rho Q_{\mathrm{S}}\right) \\
\boldsymbol{K}_{\mathrm{S}} \rho=-k_{\mathrm{S}} Q_{\mathrm{S}} \rho Q_{\mathrm{S}}
\end{gathered}
$$

Here $k_{\mathrm{S}}$ is the chemical rate constant for singlet pairs and $Q_{S}$ the projection operator, projecting onto the radical pair singlet spin subspace.

The anticommutator (eq 164a) was introduced by Johnson and Merrifield ${ }^{73}$ to describe spin-selective triplet-triplet annihilation and has been adopted for spin-dependent radical pair reactions in CIDNP theory by Tomkiewicz et al. ${ }^{699}$ and by Evans et al. ${ }^{700}$ The second form (eq 164b) has been advocated by Pedersen and Freed. ${ }^{701}$ Both forms conserve the Hermitian property of the density matrix. However, as has been shown by Haberkorn ${ }^{702}$ the second form (eq 164b) may lead to physically meaningless results for the density matrix (negative occupation numbers of quantum states). A linear combination of the form

$$
\boldsymbol{K}_{\mathrm{S} \rho}=-k_{1} / 2\left[Q_{\mathrm{S}}, \rho\right]_{+}+k_{2} Q_{\mathrm{S}} \rho Q_{\mathrm{S}}
$$

may be used, however, without such problems, ${ }^{702}$ if the following condition is obeyed:

$$
k_{1} \geq k_{2} \geq 0
$$

Expression 165 takes into account spin-exchange processes in the radical pair, which lead to singlet-triplet phase randomization. When this expression is used, the effective recombination rate constant for singlet radical pairs is $\left(k_{1}-k_{2}\right)$.

(a) SLEs for Discrete Radical Pair Sites (Exponential SLEs). The simplest approach to a simulta- neous treatment of spin motion and recombination is to consider a model with the radical pair fixed at a certain distance where recombination and dissociation compete as first-order rate processes (the so-called "exponential" model ${ }^{52}$ ). Such a model may be physically realistic under solid-state-like conditions, e.g., for radical pairs produced in photosynthetic reaction centers (cf. ref 511, 532-534, 707, and 709). However, they have been also applied to describe the situation for biradicals ${ }^{129,703}$ or radical pairs in liquid solutions (cf. ref $157,671,699,704,706$, and 708).

A typical form of the one-site radical pair SLE with different rates of singlet and triplet recombination is given by ${ }^{157,302}$

$$
\rho=-i[H, \rho]_{-}-k_{\mathrm{D}} \rho-\left(k_{\mathrm{S}} / 2\right)\left[Q_{\mathrm{S}}, \rho\right]_{+}-\left(k_{\mathrm{T}} / 2\right)\left[Q_{\mathrm{T}}, \rho\right]_{+}
$$

In Table 19 are listed a number of references where such types of equations or their two-site extensions are treated. The table provides a shorthand survey of several characteristic features of these treatments (for other examples, including the effect of microwave fields in the spin Hamiltonian, see below (RYDMR section)). Most solutions have been worked out for overall recombination yields only. These are obtained according to

$$
Y_{\mathrm{S}(\mathrm{T})}=k_{\mathrm{S}(\mathrm{T})} \operatorname{Tr} \int_{0}^{\infty} Q_{\mathrm{S}(\mathrm{T})} \rho(t) \mathrm{d} t=k_{\mathrm{S}(\mathrm{T})} \operatorname{Tr}\left\{Q_{\mathrm{S}(\mathrm{T})} \tilde{\rho}\right\}
$$

so that it is only necessary to determine the time-integrated density matrix $\tilde{\rho}$. Time integrating eq 167 yields a system of linear equations that are of the type of the Liapunov matrix equation: ${ }^{533}$

$$
\boldsymbol{B} \tilde{\rho}+\tilde{\rho} \boldsymbol{B}^{+}=\rho(0)
$$

for which efficient numerical solution procedures have been reported. ${ }^{710}$

The conservation of the $z$ component (parallel to $\mathbf{B}_{0}$ ) of the total spin and, in case of zero field, also the conservation of the absolute value of total spin allows decomposition of the coupled system of equations (eq $169)$ into several systems of reduced dimension. However, for the general-field case, analytical solutions still have been obtained for radical pairs with one nuclear spin $(I=1 / 2)$ only (cf. Table 19). In the zero-field case a general compact analytical solution has been obtained for an arbitrary number of nuclear spins $I$, where all have the same coupling constant. ${ }^{709}$

A general perturbational solution, both, stationary and time dependent, has been derived by Lendi. ${ }^{705} \mathrm{He}$ considered a SLE in the form

$$
\begin{array}{r}
\rho=\left(-i\left[H_{0}, \rho\right]_{-}+\boldsymbol{L}_{0} \rho\right)+\left(-i\left[H_{1}, \rho\right]_{-}+\boldsymbol{L}_{1} \rho\right)+B= \\
\boldsymbol{P} \rho+\boldsymbol{Q} \rho+B
\end{array}
$$

where the terms in the first parentheses define the unperturbed part, those in the second ones contain the perturbation, and the operator $B$ describes the continuous production of radical pairs. The stochastic superoperator $\boldsymbol{L}_{0}$ must be diagonal in the basis diagonalizing $H_{0}$ in the sense that

$$
\left(\boldsymbol{L}_{0}\right)_{i k}^{l m}=\delta_{l i} \delta_{m k} l_{i k}
$$

The solution up to second order is provided in terms of the matrix elements of the superoperators $\boldsymbol{P}$ and $\boldsymbol{Q}$. 
TABLE 19. Theory of Radical Pair Mechanism: Applications of Exponential Stochastic Liouville Equations

\begin{tabular}{|c|c|c|c|c|c|c|c|}
\hline year & ref & $\begin{array}{l}\text { no. of } \\
\text { sites }\end{array}$ & $\begin{array}{c}\text { nuclear spins } \\
I_{1}, I_{2} \\
\end{array}$ & $\begin{array}{c}\text { other } \\
\text { interactions }{ }^{a}\end{array}$ & $\begin{array}{l}\text { magnetic } \\
\text { field }\end{array}$ & $\begin{array}{c}\text { type of } \\
\text { solution }^{b}\end{array}$ & remarks \\
\hline \multirow[t]{2}{*}{1972} & Groff et al. ${ }^{465}$ & 1 & $1 / 2,0$ & & var & A & $c$ \\
\hline & Tomkiewicz et al. 699 & 1 & & & $\infty$ & A & $d$ \\
\hline \multirow[t]{2}{*}{1974} & Groff et al. ${ }^{466}$ & 1 & $1 / 2,0$ & $J, D$ & var & $\mathrm{A}^{e}$ & $c$ \\
\hline & Atkins and Evans ${ }^{703}$ & 2 & $1 / 2,0$ & $J$ & $\operatorname{var}^{f}$ & $\ddot{A}$ & $d$ \\
\hline \multirow[t]{2}{*}{1976} & Michel-Beyerle et al. ${ }^{167}$ & 1 & $1 / 2,0$ & & $\begin{array}{l}0, \infty \\
\operatorname{var}\end{array}$ & $\begin{array}{l}\mathrm{A} \\
\mathrm{N}\end{array}$ & \\
\hline & Schulten et al. ${ }^{302}$ & 1 & $\Delta \omega^{x}$ & & $\infty$ & A & $p$ \\
\hline \multirow[t]{2}{*}{1977} & Sarvarov $^{704}$ & 1 & & relax $^{8}$ & & A & \\
\hline & Lendi $^{705}$ & 1 & $h$ & $h$ & var & $\mathrm{P}$ & $h, p$ \\
\hline 1978 & Werner et al. ${ }^{532}$ & $\begin{array}{l}2 \\
2\end{array}$ & $1 / 2,1 / 2$ & $j$ & $\operatorname{var}$ & $\begin{array}{l}\mathrm{N} \\
A^{e}\end{array}$ & $j$ \\
\hline \multirow[t]{3}{*}{1979} & Haberkorn and Michel-Beyerle ${ }^{633}$ & 1 & $1 / 2,0$ & $J$ & $\begin{array}{l}\operatorname{var}^{\prime} \\
0, \infty\end{array}$ & $\mathrm{A}^{e}$ & $\begin{array}{l}m \\
j\end{array}$ \\
\hline & Haberkorn et al. ${ }^{534}$ & 2 & & $J^{i}$ & var & $n$ & $j$ \\
\hline & Müller et al. ${ }^{176}$ & $2^{\circ}$ & 1,0 & $s$ & var & A & $\mathrm{c}$ \\
\hline 1980 & Kubarev and Shustov ${ }^{706}$ & 2 & $\Delta \omega^{x}$ & $f^{i}$ & $\infty$ & A & \\
\hline \multirow[t]{3}{*}{1982} & Salikhov and Sarvarov ${ }^{671}$ & $\mathrm{n}^{q}$ & $\begin{array}{l}1 / 2,0 \\
1 / 2,1 / 2\end{array}$ & & $\infty$ & A & $d$ \\
\hline & Papier et al. ${ }^{467}$ & $3^{q, r}$ & 1,0 & $s$ & var & A & $c$ \\
\hline & Tang and Norris ${ }^{707}$ & 1 & $1 / 2,1 / 2$ & $J, D$ & var & $\mathrm{N}^{p}$ & $j$ \\
\hline 1982 & Boxer et al. & 1 & $\Delta \omega^{x} / 2$ & $J D$ & $\mathrm{var}^{b b}$ & A & $a a$ \\
\hline 1983 & Salikhov and Mikhailov ${ }^{708}$ & 2 & 1,0 & $J$ & 0 & A & \\
\hline \multirow[t]{2}{*}{1984} & Stich et al. ${ }^{129}$ & 1 & $1 / 2,0$ & $J$ & var & $\mathrm{P}$ & $u$ \\
\hline & Hoff and Hore ${ }^{709}$ & 1 & $1 / 2,0$ & $J^{i}$ & & $\mathrm{~N}$ & $j, v$ \\
\hline 1985 & Chidsey et al. ${ }^{511}$ & $2^{c c}$ & $w$ & $w$ & $w$ & A & $j, w$ \\
\hline 1986 & Schulten and Windemuth ${ }^{523}$ & 2 & $1 / 2,0$ & & $\operatorname{var}^{l}$ & $\mathrm{~A}^{e}$ & $m$ \\
\hline 1987 & Goldstein and Boxer ${ }^{764}$ & $2^{c c}$ & $B_{\mathrm{A}}, B_{\mathrm{B}}^{y}$ & & 0 & $\mathrm{~N}$ & $j, z$ \\
\hline & & & & & $\operatorname{var}^{b b}$ & A & \\
\hline
\end{tabular}

${ }^{a} J$, exchange interaction; $D$, electron-spin dipolar interaction. ${ }^{b} \mathrm{~A}$, analytical, exact closed form; $\mathrm{P}$, perturbational; $\mathrm{N}$, numerical.

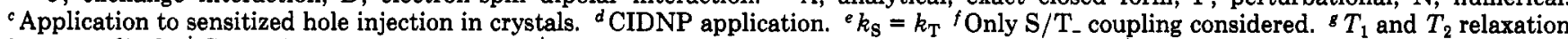
in one radical. ${ }^{h}$ General operator formalism. ${ }^{i}$ Exchange interaction with third radical included. ${ }^{j}$ Application to photosynthetic reaction centers. ${ }^{k}$ Anisotropic hfc included. 'Evaluation of orientational dependence. ${ }^{m}$ Model for biomagnetic sensory mechanism. ${ }^{n}$ Transformation to effective one-site radical pair SLE. ${ }^{\circ}$ Second site corresponds to free 2-D diffusion. ${ }^{p}$ Also with time-dependent solution. ${ }^{q}$ To simulate electron hopping. $r$ Third site corresponds to diffusing hole trapped on surface. ${ }^{s}$ Variable electric field affects escape time of hole from surface to volume of crystal. ${ }^{t}$ With averaging over discrete Gaussian distribution of hfc constants. ${ }^{u}$ Biradical in the gas phase. ${ }^{v}$ Calculation of electron spin polarization. ${ }^{w}$ Derivation of general relation between ${ }^{3} \mathrm{P}$ decay rate and yield; cf. text. ${ }^{x}$ Applies to general nuclear spin state, since this is a constant of motion under high-field conditions. ${ }^{y}$ Semiclassical approach; $B_{\mathrm{A}}$ and $B_{\mathrm{B}}$ are effective hyperfine fields at radicals $A$ and $B$. ${ }^{2}$ Generation of nuclear spin polarization and kinetic effects derived from it. ${ }^{a c}$ Accounting for anisotropic interactions and performing angular average. ${ }^{b b} \mathrm{P}^{+\bullet} \mathrm{I}^{-\bullet}$ and ${ }^{3} \mathrm{P} \mathrm{I}$. ${ }^{c c}$ Variable field, but only in the high-field limit.

Lendi's formalism has been applied by Stich et al. ${ }^{129}$ to analyze a MFE on a biradical decay in the gas phase. They considered a one-nucleus $(I=1 / 2)$ model radical pair with $H_{0}$ and $\boldsymbol{L}_{0}$ given by

$$
\begin{aligned}
& H_{0}=g \mu_{\mathrm{B}} \mathbf{B}_{0}\left(\boldsymbol{S}_{1}+\boldsymbol{S}_{2}\right)-J\left(1 / 2+2 \boldsymbol{S}_{1} \boldsymbol{S}_{2}\right) \\
& \boldsymbol{L}_{0} \rho=-\left(k_{\mathrm{S}} / 2\right)\left[Q_{\mathrm{S}}, \rho\right]_{+}-\left(k_{\mathrm{T}} / 2\right)\left[Q_{\mathrm{T}}, \rho\right]_{+}
\end{aligned}
$$

The perturbational terms were

$$
\begin{gathered}
H_{1}=a \boldsymbol{S}_{1} \boldsymbol{I}_{1} \\
\boldsymbol{L}_{1}=0
\end{gathered}
$$

Stich et al..$^{129}$ have determined the parameters $J, k_{S}$, and $k_{\mathrm{T}}$ by comparing the perturbational solution with experiment. It is, however, doubtful whether the simple one-site model can satisfactorily account for the fluctuations of the exchange interaction caused by the random motion of the biradical end-to-end distance.

For internally mobile radicals and freely diffusing radicals in solution the one-site exponential model is certainly not adequate since it has turned out that spin motion occurs essentially at separations where recombination is not yet feasible. Therefore, in order to keep the advantage of the mathematical simplicity of the exponential model, radical pair models with two sites or more than two sites have been introduced $^{532,534,671,703,706,708}$ with first-order rate processes describing transitions between the different radical pair sites. Atkins and Evens ${ }^{703}$ treated the CIDNP problem in biradicals using a two-site exponential model. However, even this two-site model turned out to be not really sufficient to account for the dynamics of distance-dependent exchange interaction in such systems (cf. ref 346 and 695).

Exponential SLE models have been widely applied for the analysis of magnetic field effects in photosynthetic reaction centers (cf. also the review by Boxer et al. ${ }^{45}$ ). Most of these treatments have taken exchange interaction into account. As an example of an exact analytical solution to eq 167 , with $k_{\mathrm{D}}=0$ and $H$ given by the sum of eq 172 and 174a (i.e., for one nuclear spin with $I=1 / 2$ and hfc constant $a$ ), we quote the result of Haberkorn and Michel-Beyerle ${ }^{533}$ for the zero-field and high-field cases (in the original paper $-2 J$ is used in place of $J$ in eq 172; eq 175 a is adapted correspondingly):

$$
\begin{array}{r}
\mathrm{s}_{Y_{\mathrm{T}}\left(B_{0}=0\right)=} 3 a^{2} k_{\mathrm{T}}\left(k_{\mathrm{S}}+k_{\mathrm{T}}\right) /\left\{\left[3 a^{2}+4 k_{\mathrm{S}} k_{\mathrm{T}}\right] \times\right. \\
\left.\left(k_{\mathrm{S}}+k_{\mathrm{T}}\right)^{2}+16 k_{\mathrm{S}} k_{\mathrm{T}}(2 J+a / 2)^{2}\right\}
\end{array}
$$

A generalization of eq $175 \mathrm{~b}$ accounting also for anisotropic hyperfine and Zeeman interaction and for 
dipolar electron spin-spin interaction has been derived by Boxer and co-workers. ${ }^{45,505}$ In this case the hyperfine coupling constant $a$ in eq $175 \mathrm{~b}$ is replaced by an effective coupling constant $\omega$. The corresponding relation was further generalized to account also for the situation of a radical pair with many nuclear spins by integrating the yield ${ }^{S} Y_{T}$ over a Gaussian distribution of the effective coupling constant $\omega$. It must be emphasized that averaging over independent nuclear states is a rigorous approach in the high-field limit.

Results for intermediate fields have been obtained only numerically. They have been used to analyze in detail the influence of parameters $J, a, k_{\mathrm{S}}$, and $k_{\mathrm{T}}$ on the behavior of a photosynthetic reaction center radical pair. The MFD shows case 1 type behavior, and $B_{1 / 2}$ is approximately determined by the largest of the four parameters. Equations $175 \mathrm{a}, \mathrm{b}$ are easily adapted to the case $k_{\mathrm{D}} \neq 0$ by substituting $\left(k_{\mathrm{S}}+k_{\mathrm{D}}\right)$ for $k_{\mathrm{S}}$ and $\left(k_{\mathrm{T}}+\right.$ $\left.k_{\mathrm{D}}\right)$ for $k_{\mathrm{T}}$ and by multiplying the yields by $k_{\mathrm{T}} /\left(k_{\mathrm{T}}+\right.$ $k_{\mathrm{D}}$ ). In this way the equivalence of eq 175a (zero-field case) with a corresponding result obtained by Salikhov and Mikhailov ${ }^{708}$ for general values of nuclear spin $I$ can be demonstrated.

Werner et al. ${ }^{532}$ considered a more realistic model of the reaction center radical pair (cf. scheme 64 and Figure 39) where paramagnetic exchange between the intermediate radical $\mathrm{I}^{*-}$ and the prereduced primary acceptor radical $\mathrm{X}^{--}$is taken into account using the spin Hamiltonian

$$
\begin{array}{r}
H=g \mu_{\mathrm{B}} \mathbf{B}_{0}\left(\boldsymbol{S}_{1}+\boldsymbol{S}_{2}+\boldsymbol{S}_{3}\right)+a_{1} \boldsymbol{S}_{1} \boldsymbol{I}_{1}+a_{2} \boldsymbol{S}_{2} \boldsymbol{I}_{2}- \\
J_{\mathrm{PI}}\left(1 / 2+2 \boldsymbol{S}_{1} \boldsymbol{S}_{2}\right)-J_{\mathrm{IX}}\left(1 / 2+2 \boldsymbol{S}_{2} \boldsymbol{S}_{3}\right)
\end{array}
$$

Furthermore, the primary electron-transfer step with the singlet excited special pair ${ }^{1} \mathrm{P} *$ is considered to be reversible (cf. Figure 39) and is formally treated by a two-site radical pair model. The first site corresponds to ${ }^{1 \mathrm{P} *}$, where $J$ is large and $\mathrm{S} / \mathrm{T}$ phase randomization occurs. The second site is the true radical pair site ${ }^{2} \mathrm{P}^{2+} \mathrm{I}^{-}$(with spin Hamiltonian (176)) where $\mathrm{S} / \mathrm{T}$ mixing takes place. The model has been analyzed by detailed numerical calculations for two nuclear spins $I=1 / 2$, one each at $\mathrm{P}$ and $\mathrm{I}$.

Reversible formation of the special pair triplet state ${ }^{3} \mathrm{P}$ from the radical pair $\mathrm{P}^{0^{+}} \mathrm{I}^{\bullet^{-}}$has been theoretically analyzed by Chidsey et al., ${ }^{511}$ who established a general relation between the observed rate constant of triplet decay $k_{\text {obsd }}$, the rate constant $k_{\mathrm{ISC}}$ of the process ${ }^{3} \mathrm{P} \rightarrow$ ${ }^{1} \mathrm{P}_{0}$, and the yield of triplet formation ${ }^{\mathrm{s}} Y_{\mathrm{T}}$ from the radical pair obtained in the purely irreversible case:

$k_{\text {obsd }}=k_{\text {ISC }}+1 / 3 k_{\mathrm{S}}\left({ }^{\mathrm{S}} Y_{\mathrm{T}}\right) \exp (-\Delta H / k T)$

Here $\Delta H$ is the enthalpy difference between triplet and radical pair. Recently eq $177 \mathrm{a}$ has been generalized by Goldstein and Boxer ${ }^{764}$ to include the case where the radical pair $\mathrm{P}^{\bullet+} \mathrm{I}^{\bullet-}$ may undergo further electron transfer to an adjacent quinone $\left(\mathrm{P}^{\bullet+} \mathrm{I}^{\bullet-} \mathrm{Q} \rightarrow \mathrm{P}^{\bullet+} \mathrm{IQ}^{\bullet^{-}}\right)$with a rate constant $k_{Q}$ :

$$
\left[\frac{k_{\mathrm{T}} k_{\mathrm{Q}}}{k_{\mathrm{T}}+k_{\mathrm{Q}}}+\left(k_{\mathrm{T}} / 3\right)\left(\frac{k_{\mathrm{S}}+k_{\mathrm{Q}}}{k_{\mathrm{T}}+k_{\mathrm{Q}}}\right)\left({ }^{\mathrm{s}} Y_{\mathrm{T}}\right)\right] \exp (-\Delta H / k T)
$$

Haberkorn et al. ${ }^{534}$ distinguished two sites of the intermediate electron acceptor I, one closer and the other more distant to the special pair. This model was discussed as a possibility to reconcile the fast rate of forward electron transfer with the rather low value of the exchange energy $J$ evaluated from the magnetic field dependence of ${ }^{\mathrm{S}} Y_{\mathrm{T}}$. Such a discrepancy arises if it is assumed that the matrix element for forward electron transfer $\left({ }^{1} \mathrm{P} * \mathrm{I} \rightarrow \mathrm{P}^{+} \mathrm{I}^{-}\right)$and the exchange energy in the radical pair originate by pure through-space interactions. Meanwhile, experimental evidence has been obtained $\mathrm{d}^{515}$ pointing to the important role of superexchange, whereby the energy of virtual orbitals of intervening molecules comes into play. This yields different values for the relevant exchange integrals in the radical pair and in the matrix element of forward electron transfer. Thus the model of Haberkorn et al. ${ }^{534}$ seems to have become obsolete. However, the theoretical method for treating the two-site radical pair model is still of interest. It was shown that the coupled stochastic Liouville equations for $\mathrm{RP}_{\mathrm{C}}$ and $\mathrm{RP}_{\mathrm{D}}$ in their time-integrated forms may be transformed to an effective one-site SLE which allows reinterpretation of the parameters $J, k_{\mathrm{S}}$, and $k_{\mathrm{T}}$ in terms of the parameters $J, k_{\mathrm{S}}$, and $k_{\mathrm{T}}$ of the close radical pair and of the transition rate constants for interconversion between the two radical pair sites.

A remarkable extension of the two-site radical pair model has been developed by Charlé, Willig, and coworkers ${ }^{176}$ for the situation of dye-sensitized hole injection into molecular crystals. Here site 1 of the radical pair is a so-called bound pair of an electron, localized at the semireduced adsorbed sensitizer, and an electron hole in an adjacent molecule of the crystal. First-order ("exponential") dissociation of the site-1 radical pair yields the site-2 radical pair, where the hole undergoes two-dimensional diffusion along the surface, since its image charge in the surrounding electrolyte prevents fast escape into the volume of the crystal, also described by a first-order rate process. Spin motion occurs mainly in the site-2 situation, i.e., during the two-dimensional diffusion of the hole, where it is described by a spin evolution superoperator $\boldsymbol{M}\left(t-t^{\prime}\right)$. Returning to site 1 is described by a reencounter function $f\left(t-t^{\prime}\right)$ adapted to two-dimensional diffusion and containing an exponential factor for the escape process into the volume of the crystal (rate constant $k_{\text {esc }}$ ). The combined spin evolution reencounter superoperator thus becomes

$$
\boldsymbol{E}\left(t-t^{\prime}\right)=\boldsymbol{M}\left(t-t^{\prime}\right) f\left(t-t^{\prime}\right)
$$

The density matrix equation of motion of site- 1 radical pairs assumes the form of an integrodifferential equation:

$$
\dot{\rho}=L_{\text {int }} \rho+k_{-} \int_{0}^{t} \boldsymbol{E}\left(t-t^{\prime}\right) \rho\left(t^{\prime}\right) \mathrm{d} t^{\prime}
$$

$\boldsymbol{L}_{\text {int }}$ is the Liouville superoperator describing spin motion and kinetics of the radical pair in site 1 , which decays into site 2 with a rate constant of $k_{-}$. Laplace transforming and letting $s \rightarrow 0$ yields

$$
\rho(0)=-\boldsymbol{L}_{\text {int }} \hat{\rho}-k_{-} \boldsymbol{E} \hat{\rho}
$$

a matrix equation that may be solved by standard methods. The elements of the superoperator $\boldsymbol{E}$ are given by

$$
E_{m n}^{k l}=\hat{f}\left(k_{\mathrm{esc}}+i \omega_{m n}\right) \delta_{m n} \delta_{k l}
$$


where $\omega_{m n}$ are the frequency eigenvalues of the site- 2 spin Hamiltonian and $\hat{f}(s)$ is the Laplace transform of the reencounter function $f(t)$ for two-dimensional diffusion.

The method is actually a combination of the exponential SLE with the assembling method of the last section. It has been extended to include also a third, discrete site, where the two-dimensional diffusion of the hole is interrupted by being fixed for a while in a shallow surface trap. ${ }^{467}$

The exponential SLE has been used in several cases to study anisotropic interactions in photosynthetic reaction centers, e.g., electron spin dipolar interaction ${ }^{93,707}$ or $g$ tensor and hyperfine tensor anisotropies. ${ }^{505}$

Schulten and co-workers ${ }^{522,523}$ used a two-site radical pair model with one nuclear $\operatorname{spin}(I=1 / 2)$ in order to assess the influence of magnetic anisotropy of hyperfine coupling in oriented systems. It was found that even in the earth's magnetic field resonable angular dependences of product yields would ensue so that the model could be discussed as the basis for a biometric sensory mechanism for living organisms finding their direction with the help of the earth's magnetic field. Hoff and Lous ${ }^{514}$ suggested that anisotropic electron spin-spin interaction might be considered as a potential principle for biomagnetic sensing.

As mentioned above, in prereduced photosynthetic reaction centers paramagnetic exchange between the radical pair $\mathrm{P}^{\circ+} \mathrm{I}^{\circ-}$ and $\mathrm{X}^{*-}$ is of importance for the magnetic field dependence of triplet formation, but also in spin polarization of the prereduced primary acceptor. This has been theoretically investigated in some detail by Hoff and Hore ${ }^{709}$ using the exponential SLE formalism.

The effect of paramagnetic exchange with a nearby doublet state has been also considered in a two-site model by Kubarev and Shustov. ${ }^{706}$ According to their reasoning, the effect of paramagnetic exchange on the yield of escape may be much larger than in the most favorable case of the $\Delta g$ mechanism in a one-site pair. This could facilitate the detection of "latent" radical pairs in biological systems.

The influence of paramagnetic relaxation on singlet and triplet recombination yields of radical pairs has been modeled by Sarvarov ${ }^{704}$ in the framework of the one-site exponential SLE. For the case that spin relaxation is only efficient in one of the radicals, analytical expressions have been obtained as follows:

$$
\begin{aligned}
& { }^{\mathrm{s}} Y_{\mathrm{S}}= \\
& \quad\left(k_{\mathrm{S}} \tau_{\mathrm{c}} / 4 \Delta\right)\left[4+3 \tau_{\mathrm{c}} / T_{1 \mathrm{~A}}+2 \tau_{\mathrm{c}} / T_{2 \mathrm{~A}}+\tau_{\mathrm{c}}{ }^{2} / T_{1 \mathrm{~A}} T_{2 \mathrm{~A}}\right]
\end{aligned}
$$

$$
{ }^{\mathrm{T}} Y_{\mathrm{S}}=\left(k_{\mathrm{S}} \tau_{\mathrm{c}} / 4 \Delta\right)\left[\tau_{\mathrm{c}} / 3 T_{1 \mathrm{~A}}+2 \tau_{\mathrm{c}} / 3 T_{2 \mathrm{~A}}+\tau_{\mathrm{c}}{ }^{2} / T_{1 \mathrm{~A}} T_{2 \mathrm{~A}}\right]
$$

with

$$
\begin{aligned}
& \Delta=\left(1+\tau_{\mathrm{c}} / T_{1 \mathrm{~A}}\right)\left(1+\tau_{\mathrm{c}} / T_{2 \mathrm{~A}}\right)+ \\
& k_{\mathrm{S}} \tau_{\mathrm{c}}\left(1+3 \tau_{\mathrm{c}} / 4 T_{1 \mathrm{~A}}+\tau_{\mathrm{c}} / 2 T_{2 \mathrm{~A}}+\tau_{\mathrm{c}}{ }^{2} / 4 T_{1 \mathrm{~A}} T_{2 \mathrm{~A}}\right)
\end{aligned}
$$

$k_{\mathrm{S}}$ is the rate constant of singlet pair recombination, $\tau_{\mathrm{c}}$ is the mean cage time, and $T_{1 \mathrm{~A}}$ and $T_{2 \mathrm{~A}}$ are the respective relaxation times of one of the radicals in the pair considered to have a particularly short spin relaxation, e.g., small inorganic radicals.

The problem of electron hopping between radical ions and neutral donor and/or acceptor molecules has been treated by Salikhov and Sarvarov ${ }^{671}$ for the high-field limit ( $\mathrm{S} / \mathrm{T}_{0}$ mixing only) using an $n$-site exponential SLE model. In one radical pair site nuclear spin states are conserved in high magnetic fields; however, on electron hopping the radical pair may find itself in a different nuclear spin state. Thus each nuclear spin state may be considered as a separate radical pair site.

The semiclassical treatment of hyperfine coupling has been combined with the stochastic Liouville formalism by Goldstein and Boxer. ${ }^{764}$ Here the nuclear spin states are assumed to be unchanged during electron spin motion, which is strictly valid only in the high-field limit. The nuclear states are represented by equivalent effective magnetic fields in each radical. For each fixed pair of hyperfine fields $\left(B_{\mathrm{A}}, B_{\mathrm{B}}\right)$ on radicals $\mathrm{A}$ and $\mathrm{B}$ the SLE is solved according to the method of Haberkorn and Michel-Beyerle. ${ }^{533}$ The solutions depend on the $\mathrm{S} / \mathrm{T}$ mixing frequency

$$
\omega=g \mu_{\mathrm{B}}\left|\mathbf{B}_{\mathrm{A}}-\mathbf{B}_{\mathrm{B}}\right| / \hbar
$$

and are averaged over the various distributions $R(\omega)$. The work focuses attention especially on the production and consequences of nuclear spin polarization (expressed in the particular form of $R(\omega)$ ) in photosynthetic reaction centers. Two mechanisms of such nuclear spin polarization are pointed out:

(a) Under single-excitation conditions sorting of nuclear spin states in the branching of the radical pair ${ }^{1}\left(\mathrm{P}^{{ }^{++}} \mathrm{I}^{\bullet-}\right)$ to form $\mathrm{PI}$ and ${ }^{3} \mathrm{PI}$ leads to differential nuclear spin polarization of PI and ${ }^{3} \mathrm{PI}$. If ${ }^{3} \mathrm{PI}$ decays to PI, however, prior to nuclear spin relaxation, net polarization will be zero.

(b) Under stationary irradiation conditions the faster nuclear spin relaxation in the paramagnetic ${ }^{3} \mathrm{PI}$ state leads to a stationary nuclear polarization of PI. This polarization causes an appreciable effect on the saturation value of $\left[{ }^{3} \mathrm{PI}\right]$ as a function of pump light intensity. A decrease of nuclear polarization by resonant $\mathrm{RF}$ absorption is predicted to give rise to significant effects on reaction yields and kinetic parameters, thus enabling "RYDNMR"-type experiments to be performed.

(b) Stochastic Liouville Equations with Continuous Diffusion. In this section we will deal with publications where methods of direct solutions of the full SLE in the form given in eq 120 are described. Here translational and rotational diffusion of the radicals are modeled by a stochastic operator $\Gamma(\mathbf{r}, \Omega)$, which is usually restricted to configuration space; i.e., it is assumed that relaxation times of linear and angular molecular momentum are infinitely short. This type of stochastic motion is tantamount to assuming the so-called sudden perturbation limit of time-dependent quantum theory. As a consequence, SLEs with this term would not be adequate to describe slow passage of radical pairs through domains where the energy eigenstate basis changes. In such cases quantum theory would predict adiabatic changes of quantum states. In order that eq 120 is applicable, the condition for a sudden crossing from the region where hyperfine coupling determines the eigenstates of the spin Hamiltonian to the region where they are defined by exchange interaction must be fulfilled. In simplified form this condition may be stated as 
TABLE 20. Theory of the Radical Pair Mechanism: Applications of SLEs with Continuous Diffusion

\begin{tabular}{|c|c|c|c|c|c|c|c|c|c|}
\hline year & ref & $\begin{array}{c}\text { S/T } \\
\text { coupling }^{a}\end{array}$ & $\begin{array}{l}\text { exchange } \\
\text { interaction }^{b}\end{array}$ & $\begin{array}{l}\text { magnetic } \\
\text { field }\end{array}$ & $\begin{array}{l}\text { max order of } \\
\text { DM blocks }\end{array}$ & $\begin{array}{l}\text { diffusion } \\
\text { potential }^{d}\end{array}$ & $\begin{array}{l}\text { boundary } \\
\text { conditions }^{e}\end{array}$ & $\begin{array}{l}\text { method of } \\
\text { solution }\end{array}$ & remarks $^{8}$ \\
\hline 1972,1973 & Pedersen and Freed ${ }^{711,712}$ & $\omega_{\mathrm{N}}$ & e, $\delta$ & $\infty$ & 2 & 0 & $\mathrm{sp}^{a a}$ & $\Delta r$ & $g$ \\
\hline \multirow[t]{2}{*}{1973} & Pedersen and Freed ${ }^{701}$ & $\omega_{\mathrm{N}}$ & $\mathrm{e}$ & $\infty$ & 2 & $J$ & $\mathrm{sp}^{a a}$ & $\Delta r$ & $g$ \\
\hline & Evans et al. ${ }^{700}$ & $\omega_{\mathrm{N}}, R$ & $\delta$ & $\infty$ & 2 & 0 & $\mathbf{s p}^{h}$ & A & $g, i$ \\
\hline 1974 & Pedersen and Freed ${ }^{713}$ & $\omega_{N}$ & $\mathrm{e}$ & $\infty$ & 2 & $C, J$ & $\operatorname{sp}^{a a}$ & $\Delta r$ & $i$ \\
\hline \multirow[t]{3}{*}{1975} & Pedersen and Freed ${ }^{714}$ & $\omega_{\mathrm{N}}$ & e & $\infty$ & 2 & $\mathrm{H}^{j}$ & $\mathrm{sp}^{a a}$ & $\Delta r$ & $g, i$ \\
\hline & Evans and Lawler ${ }^{648}$ & $1 / 2$ & $\delta$ & var & 3 & 0 & $\mathrm{sp}^{a a}$ & $\mathrm{AE}$ & $i$ \\
\hline & Salikhov et al. ${ }^{715}$ & $\omega_{N}$ & 0 & $\infty$ & 2 & 0 & rd & A & \\
\hline \multirow[t]{2}{*}{1976} & Evans $^{716}$ & $\omega_{\mathrm{N}}$ & $\delta$ & $\infty$ & 2 & 0 & $\mathrm{sp}^{l}$ & A & $g, i$ \\
\hline & Sarvarov and Salikhov ${ }^{717}$ & $\frac{\Delta g}{1 / 2}$ & 0 & var & $\begin{array}{l}2 \\
3\end{array}$ & 0 & $\mathrm{rd}^{m}$ & $\mathrm{~A}$ & $m$ \\
\hline \multirow[t]{3}{*}{1977} & Werner et al. ${ }^{643}$ & $1 / 2,1 / 2$ & $J^{0}$ & var & 6 & $C$ & $\mathrm{sp}^{\alpha a}$ & $\Delta r, \Delta t$ & \\
\hline & Haberkorn ${ }^{602}$ & $p$ & $\delta$ & var & $p$ & 0 & $\mathrm{sp}^{h}$ & $\mathrm{AE}$ & $p$ \\
\hline & Haberkorn $^{721}$ & $p$ & $\delta$ & var & $p$ & 0 & $\mathrm{sp}^{h}$ & $\mathrm{AE}(t)$ & $q$ \\
\hline 1977,1978 & de Kanter et al. ${ }^{346,718-720}$ & $1 / 2, R$ & $\mathrm{e}$ & var & 8 & $P^{n}$ & $\mathrm{rd}$ & $\Delta r$ & $i, n$ \\
\hline 1979,1980 & Adrian and Monchick ${ }^{722,723}$ & $1 / 2$ & $\mathrm{e}$ & var & $2^{r}$ & 0 & sp & $\mathrm{AE}$ & $g, i$ \\
\hline \multirow[t]{2}{*}{1979} & Zientara and Freed 724 & $1 / 2$ & e & var & 3 & 0 & $\mathrm{sp}^{a a}$ & $\Delta r$ & $g, i$ \\
\hline & Zientara and Freed ${ }^{725}$ & $\omega_{\mathrm{N}}$ & $\mathrm{e}, \theta^{t}$ & $\infty$ & 2 & 0 & $\mathrm{sp}^{a a}$ & $\Delta r, \theta^{u}$ & $g, i$ \\
\hline \multirow[t]{2}{*}{1982} & Shushin 726 & $\omega_{\mathrm{N}}$ & $\mathrm{e}^{r}$ & $\infty$ & 2 & $C^{v}$ & rd & $\mathrm{AP}$ & \\
\hline & Sarvarov et al..$^{668}$ & $\omega_{\mathrm{N}}$ & $w$ & $\infty$ & 2 & 0 & $w$ & A & $i, k$ \\
\hline 1984 & Mints and Pukhov ${ }^{727}$ & $\Delta g, R^{x}$ & 0 & var & $2.5^{y}$ & 0 & $\mathrm{rd}$ & A & \\
\hline 1985 & Lüders and Salikhov ${ }^{728}$ & $\Delta g$ & 0 & var & 2 & 0 & $\mathrm{rd}^{z}$ & A & $z$ \\
\hline 1986 & Shushin ${ }^{729}$ & $p$ & $p$ & var & $p$ & $C$ & $p$ & AP & \\
\hline
\end{tabular}

${ }^{0} \omega_{\mathrm{N}}, \mathrm{S} / \mathrm{T}_{0}$ coupling with fixed nuclear spin states; $R$, paramagnetic relaxation; $\Delta g$, Zeeman coupling of $\mathrm{S}$ and $\mathrm{T}_{0}$; figures refer to nuclear spins present. ${ }^{b} \mathrm{e}, J_{0} \exp (-\alpha r) ; \delta, \delta(r-a)\left(a\right.$ is the reaction diameter). ${ }^{c}$ Block diagonalization of $\rho$ in selection-rule-adapted basis. ${ }^{d} \mathrm{C}$, Coulombic potential; $J$, exchange interaction included in diffusion operator. ${ }^{e}$ sp, specular boundary condition; rd, spin-selective radiation boundary condition at $r=a$ ( $a$ is the reaction diameter). ' $\Delta r, \Delta t$, finite differences; $\mathrm{A}$, analytical; $\mathrm{N}$, numerical; $\mathrm{AE}$, analytical method, affording result in a closed-form expression; AP, analytical, perturbational approach. ${ }^{8}$ CIDEP calculations. ${ }^{h}$ Reflecting walls at $r=0$ and $r=a, \delta$-type $r$-dependent singlet reaction at $r=a .{ }^{i}$ CIDNP calculations. ${ }^{j} \mathrm{H}$, hydrodynamic effects, applying Oseen's tensor. ${ }^{k}$ Consecutive radical pair. ' Reflection at $0<b \leq a$. ${ }^{m}$ Treatment of homogeneous radical recombination, boundary condition at infinity $\rho(r \rightarrow \infty)=\sigma_{\mathrm{A}} \times$ $\sigma_{\mathrm{B}} .{ }^{n}$ Polymer chain dynamics, biradical. ${ }^{\circ}$ Various step models for distance dependence of $J . \quad{ }^{p}$ General theory. ${ }^{q}$ General time-dependent theory. ${ }^{r} \mathrm{~S} / \mathrm{T}_{ \pm}$mixing only. "Unless otherwise stated (CIDNP, CIDEP), radical recombination yields are calculated. 'Angular dependence of exchange interaction described by a $\cos \theta$ term. "Orthogonal expansion in Legendre polynomials. ${ }^{~}$ Exponential $J(r)$ without Coulombic interaction or Coulombic interaction with "hard-sphere" type exchange interaction. ${ }^{w}$ Radiation boundary condition for $\rho_{\mathrm{Ss}}$, absorbing

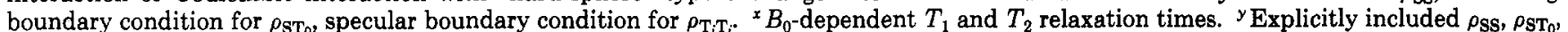
$\rho_{\mathrm{T}_{0} \mathrm{~S}}, \rho_{\mathrm{T}_{0} \mathrm{~T}_{0}}, \rho_{\mathrm{T}_{+} \mathrm{T}_{+}}$, and $\rho_{\mathrm{T}_{-} \mathrm{T}_{-}}{ }^{z}$ Boundary conditions appropriate for micelles: spin-selective radiation boundary condition at $r_{\min }=a$, general radiation boundary condition for escape at $r_{\max }$. ${ }^{a a}$ In the finite-difference methods spin-selective reaction is added as an independent first-order process in certain $\Delta r$ increments.

$$
1 / \tau_{\mathrm{D}} \equiv D / a^{2} \gg \omega_{\mathrm{hfc}}
$$

where $a$ is the radical pair encounter distance and $\omega_{\mathrm{hfc}}$ a frequency corresponding to the typical order of hyperfine-coupling strength. In this so-called low-frequency limit ${ }^{648}$ the solutions of eq 120 can be expected to give an adequate description of the physical situation.

Possibilities for generalizing the description of stochastic motion by adding linear and angular velocities as stochastic variables have been indicated by Freed and Pedersen. ${ }^{18}$

Since possible orientational dependence of radical reactivity and exchange interaction is usually neglected (for an exception cf. ref 725) and the reaction media are usually isotropic, the operator $\Gamma$ may be used in an $r$-dependent form only, given as ${ }^{701}$

$$
\Gamma(r) \rho(r)=D \nabla[\nabla \rho(r)+(1 / k T) \rho(r) \nabla \boldsymbol{U}(r)]
$$

Here the first term in the brackets describes the diffusional tendency to level concentration gradients and the second term describes fluxes due to potential gradients. The potential $U(r)$ could be due to Coulombic interaction in the radical pair or to exchange forces. In the latter case it would be spin-dependent and therefore it is written as a superoperator. There have also been suggestions to generalize the stochastic operator to include hydrodynamic effects on radical pair motion. ${ }^{730}$ The consequences of these in CIDMP effects have been studied theoretically by Pedersen and Freed. ${ }^{714}$
Various mathematical techniques have been employed to obtain solutions of the continuous-diffusion SLE in relation to various radical pair models. In Table 20 we present a survey of the original literature where methods of solution have been developed.

With very few exceptions (vide infra) most authors have confined themselves to obtain time-integrated solutions of the SLE. Thus it is customary to Laplace transform eq 120 and let the Laplace variable $s \rightarrow 0$. From the solution of the ordinary differential equation for $\bar{\rho}(r)$, defined as

$\bar{\rho}(r)=\lim _{s \rightarrow 0} \hat{\rho}(r, s)=\lim _{s \rightarrow 0} \int_{0}^{\infty} \rho(r, t) \exp (-s t) \mathrm{d} t$

all time-integrated effects are easily obtained.

Initial and Boundary Conditions. The initial condition usually applied assumes creation of a radical pair at some separation $r_{0}$ in some specified spin state

$$
\begin{gathered}
\rho(r, t=0)=\left(1 / 4 \pi r_{0}^{2}\right) \delta\left(r-r_{0}\right) \rho_{0} \\
\rho_{0} \propto Q_{\mathrm{S}} \\
\text { or } \quad \rho_{0} \propto Q_{\mathrm{T}} \\
\text { or } \quad \rho_{0} \propto I \\
\operatorname{Tr}\left\{\rho_{0}\right\}=1
\end{gathered}
$$

where $Q_{\mathrm{S}}, Q_{\mathrm{T}}$, and $I$ (unit operator) correspond to the 
creation of a singlet, a triplet, or a diffusional (F) radical pair, respectively.

The two types of boundary conditions used for the diffusion operator are specular (sp) and radiative (rd) conditions given by eq 187 and 188 , respectively.

$$
r^{2}\left(\frac{\partial \rho}{\partial r}\right)_{r=r_{\min }}=0 \quad \text { (specular boundary condition) }
$$

$$
D\left(\frac{\partial \rho}{\partial r}\right)_{r=a}=K(a) \rho \quad \text { (radiation boundary condition) }
$$

With specular boundary conditions the number of radical pairs is conserved, so that radical recombination has to be described by a separate term. Several authors $\mathrm{s}^{648,682,700}$ have used condition (187) at $r_{\min }=0$ and introduced a reactive sink at $r=a$, corresponding to the encounter radius. Evans ${ }^{716}$ has shown that mathematical admission of distances $r<a$, which is physically impossible, does not influence the results too drastically. The consequences of representing the exchange interaction as a $\delta$-function are more serious, but this aspect is more important in CIDNP effects than in chemical yield effects. Specular boundary conditions are usually applied in connection with finite-difference approximation methods, whereas the radiation boundary condition is usually applied in connection with analytical solutions of the continuous-diffusion SLE. Since the superoperator $\boldsymbol{K}$ represents the effect of spin-dependent recombination, not all matrix elements of $\rho$ are equally affected. In the case of exclusive singlet recombination, eq 188 is tantamount to a reflecting boundary condition for $\rho_{T_{i} T_{i}}$, whereas it corresponds to an absorbing boundary condition for $\rho_{\mathrm{SS}}$ and $\rho_{\mathrm{ST}}$ (if recombination of singlet radical pairs is diffusion controlled). In the limit $r \rightarrow \infty$ the specular boundary condition is usually employed. However, in the finite-difference methods, ${ }^{667-670,672,673}$ which have to be terminated at some $r$ $=r_{\max }$, an absorbing boundary condition

$$
\rho\left(r_{\max }, t\right)=0 \quad \text { (absorbing boundary condition) }
$$

is customary.

In the case of radical pairs in micelles it is appropriate to use the radiation boundary condition at $\mathbf{r}=\mathbf{r}_{\max }$ corresponding to the micellar radius. ${ }^{728}$

The SLE approach has also been applied to describe homogeneous recombination of radicals, ${ }^{717}$ in which case the suitable boundary condition at infinity is given by

$$
\lim _{r \rightarrow \infty} \rho(r, t)=\sigma_{\mathrm{A}}(t) \times \sigma_{\mathrm{B}}(t)
$$

with $\sigma_{\mathrm{A}}$ and $\sigma_{\mathrm{B}}$ the single radical spin density matrices.

Numerical Integration by Finite-Difference Methods. The continuous-diffusion SLE and its numerical integration by finite-difference methods have been introduced and mainly exploited by Pedersen and Freed (cf. Table 20) in the theoretical study of CIDNP and CIDEP effects. The advantage of this type of technique is that any special $r$ dependence of the Hamiltonian (exchange interaction), the reactivity superoperator $\boldsymbol{K}(r)$, and the diffusional operator $\Gamma(r)$ may be taken into account in a straightforward manner, so that no physically unreasonable assumptions have to be imposed on the radical pair models. A disadvantage is that the parameter dependence of the results has to be cast in numerical tables. Using, however, dimensionless parameters a fairly comprehensive survey of the various cases could be established. ${ }^{18}$ Although the work of Pedersen and Freed is mainly devoted to a calculation of CIDEP and CIDNP effects, the parameters $F^{*}$ and $\Lambda$, which may be combined to obtain the general recombination yield under the condition of spin motion and spin-dependent recombination probability (cf. section 5), are most valuable for those interested in magnetic field effects on chemical yields. ${ }^{52}$

In the finite-difference approximation the distance domain from $r_{\min }$, generally the radical pair encounter distance, to some $r_{\max }$ is divided into discrete $\Delta r$ increments whereby, without loss of accuracy, great improvements in computer storage and time savings can be achieved if the length of $\Delta r$ is increased in a geometric progression toward the upper limit of the $r$ range. ${ }^{724}$ Introducing $r$ discretization, the SLE is turned into a system of linear equations for the matrix elements $\hat{\rho}_{k l}\left(r_{i}, s\right)$. A critical discussion of the convergence limits with respect to parameters $\Delta r, r_{\max }$, and Laplace transform variable $s$ has been given in a review by Freed and Pedersen. ${ }^{18}$

The dimension of the linear matrix equation to be solved is determined by the coupling scheme of the $\rho$ matrix elements. In the high-field limit there is only $\mathrm{T}_{0} / \mathrm{S}$ mixing and a conservation of nuclear spin states. The complete problem is decomposed into a number of $2 \times 2$ spin matrix elements multiplied by the number of $\Delta r$ increments. In the low-field case all electronic spin states are coupled and nuclear spin states, too, are not conserved. Thus the dimension of coupled blocks of the density matrix rapidly increases with the number of nuclear spins and only rather simple model spin systems have been studied. ${ }^{346,643,724}$

Using the finite difference numerical integration method Pedersen and Freed investigated in detail the influence of spatial extent of the exchange interaction. ${ }^{712}$ This turned out to be especially important when dealing with CIDEP effects. On the other hand, it has been shown by a number of workers that for CIDNP and reaction-yield-detected magnetic field effects, the special form of exchange interaction is less important. ${ }^{643,682}$ The reason for this significant difference is that, in order to produce CIDEP weak exchange collisions must occur in the radical pair ${ }^{637}$, which is more likely with a far-reaching, fairly slowly decaying, exchange interaction.

Other aspects that have been studied concern the role of exchange and Coulombic forces as they modify the diffusional motion ${ }^{701,713}$ and hydrodynamic effects, which were accounted for by an $r$-dependent diffusional tensor (Oseen's tensor). ${ }^{714}$

Anisotropic reactivity has been taken into account by admitting one angular degree of freedom in the spatial dependence of $\rho$. A discretization in the angular subspace was achieved in terms of orthogonal expansion in a series of Legendre polynomials. ${ }^{725}$ The problem of anisotropic reactivity has also been treated by Salikhov ${ }^{656}$ however, in a much simpler scheme of two discrete rotational sites.

The first explicit results on the MFD of chemical reaction yields from numerical integration of the full SLE have been obtained by Werner et al. ${ }^{643}$ In their 
calculations an explicit time integration by a finitedifference method was also employed, yielding the time dependence of singlet and triplet recombination yields under various conditions. Diffusion in a Coulombic potential and exchange-modified spin motion were taken into account. For the zero-field case a model radical pair with one nuclear spin $I=1 / 2$ on each radical was employed, whereas in the high-field limit a summation over 625 independent solutions of $2 \times 2$ spinspace problems for the realistic pyrene $-/ \mathrm{DMA}^{+}$hyperfine coupling situations was carried out. It was found that the precise form of the exchange interaction significantly influences the recombination yields only in solvents of low polarity, where the Coulombic interaction can force the radical pair to remain in the strong-exchange region for a longer time. The influence of exchange interaction on the relative magnetic field effect on recombination yields is fairly weak. The numerical solution of the full SLE problem corroborated the results of much simpler integration procedures with an assembling of spin motion and diffusion by coupled diffusion equations (cf. section 5), thus justifying the application of the latter methods to study in detail the solvent, temperature, magnetic field, and magnetic isotope dependence of recombination yields.

De Kanter et al. ${ }^{346}$ treated spin-selective recombination of biradicals by the continuous-diffusion SLE using a restricted diffusional model to describe the time dependence of the biradical end-to-end distance distribution. In this way the problem turned out to be more tractable than using a multirotational isomeric state model. The $\Delta r$ increments were determined so as to receive equal end-to-end distribution probabilities under equilibrium chain-folding conditions. The transition rates $W_{k l}$ between adjacent $\Delta r$ elements centered at $r_{k}$ and $r_{l}$ were described by analogy with classical Brownian motion with an effective diffusion constant $D^{\prime}$ :

$$
W_{k l}=\left(D^{\prime} /\left(r_{l}-r_{k}\right)^{2}\right) \delta_{k, l \pm 1}
$$

Although $W_{k l}$ is $r$ dependent, eq 191 is in accord with the correct equilibrium distribution, because $W_{k l}=W_{l k}$. Since the Redfield relaxation matrix was included in the Liouville operator of spin motion, the whole problem could not be partitioned into independent spinsubspace problems. Thus only the simplest model radical pair with one nuclear spin $(I=1 / 2)$ was analyzed. The coupling of 20 matrix elements of the spin density matrix had to be considered for determining the CIDNP effects, which, with 15 spatial increments of polymethylene chain end-to-end distribution $\left(\mathrm{C}_{7}\right.$ biradical $)$, yielded a 302-dimensional system of linear equations which was numerically solved for variable values of $B_{0}$. Although the evaluation was not aimed at determining the MFD of overall reaction yields, the conclusion that the CIDNP results cannot be understood on the basis of a simple one-site radical pair model with an average exchange interaction is also valid for MFEs on overall quantum yields with covalently linked radical pairs as has been shown in detail by Schulten and co-workers. ${ }^{347,665,695}$

Analytical Solutions. Although diffusion equations may be solved analytically for a great variety of boundary conditions, ${ }^{731}$ the $r$-dependent mixing of spin density matrix elements by spin-dependent potentials renders a general analytical solution of eq 120 practi- cally impossible. The borderline case, however, where $r$-dependent interactions are confined to an infinitesimal range of $r$ in the form of $\delta$-functions is amenable to analytical solution. The density matrix elements may be decoupled in the potential-free regions using a spin-state basis that diagonalizes the Hamiltonian. The $\delta$-type potentials will cause dịscontinuities in the gradient of $\rho$ and may be accounted for by means of suitable boundary conditions at the boundary between the two continuous regions of $\rho$ and $\partial \rho / \partial r$.

Evans et al. ${ }^{700}$ were the first to use this method in a quantitative theory of the CIDNP effect. Then the method was adopted by Salikhov et al. ${ }^{715,717}$ and by Haberkorn. ${ }^{682,721}$ Further contributions are due to Adrian and Monchick, ${ }^{722,723}$ to Shushin, ${ }^{726,729}$ and to Mints and Pukhov. ${ }^{727}$

For an illustration of the method we shall outline the procedure used by Haberkorn. ${ }^{682}$ He used the following form of $r$-dependent operators in eq 120:

$$
\begin{gathered}
H_{\mathrm{ex}}(r)=-J(r) Q_{\mathrm{S}} \\
\boldsymbol{K}(r) \rho(r)=-1 / 2 K(r)\left[Q_{\mathrm{T}}, \rho(r)\right]_{+}
\end{gathered}
$$

with

$$
\begin{aligned}
& J(r)=J_{0} r_{0} \delta\left(r-r_{0}\right) \\
& K(r)=k_{0} r_{0} \delta\left(r-r_{0}\right)
\end{aligned}
$$

and the initial condition was

$$
\rho(r, t=0)=\rho_{0} r_{0} \delta\left(r-r_{0}\right)
$$

Applying Laplace transformation in time and Fourier transformation in space, eq 120 was transformed into a matrix equation that could be formally solved and after Fourier transforming backward yielded the following matrix equation for $\bar{\rho}\left(r_{0}\right)$ :

$$
\begin{aligned}
& \bar{\rho}_{k l}\left(r_{0}\right)= \\
& \quad Z_{k l}\left(\tau_{\mathrm{D}}, \omega_{k l}\right)\left\langle\rho_{0}+i J_{0}\left[Q_{\mathrm{S}}, \bar{\rho}\left(r_{0}\right)\right]_{-}-k_{0} / 2\left[Q_{\mathrm{T}}, \bar{\rho}\left(r_{0}\right)\right]_{+}\right\rangle_{k l}
\end{aligned}
$$

with

$$
\begin{gathered}
Z_{k l}=\left\{1-\exp \left(-q_{k l}\right)\right\} \tau_{\mathrm{D}} / q_{k l} \\
q_{k l}=2 \sqrt{i w_{k l} \tau_{\mathrm{D}}} \\
+ \\
w_{k l}=\left(\epsilon_{k}-\epsilon_{l}\right) / \hbar
\end{gathered}
$$

In eq 197 the eigenstates of the $r$-independent spin Hamiltonian with eigenvalues $\epsilon_{i}$ are used as basis states.

The analogous result to eq 197, however for singlet pair recombination only, had been derived by Evans et al. ${ }^{700}$ Only in the high-field case a closed-form analytical solution can be obtained. Since it is, however, a rather unwieldy expression, we shall quote here, in a form compatible with the symbols generally used in this section, the exact high-field results derived by Salikhov $^{715}$ for the simpler case with neglect of exchange interaction and adapted to the case with a radiation boundary condition at $r=r_{0}=a$.

$$
\begin{gathered}
{ }^{\mathrm{s}} Y_{\mathrm{S}}=\sum_{m} \Lambda_{m}(2+\delta(m)) \\
\mathrm{T}_{0} Y_{\mathrm{S}}=\sum_{m} \Lambda_{m} \delta(m)
\end{gathered}
$$

with 


$$
\begin{gathered}
\Lambda_{m}=A /\left\{[2(1+A)+\delta(m)(2+A)] \prod_{k}\left(I_{k}+1\right)\right\} \\
A=4 \pi r_{0}{ }^{2} k \tau_{\mathrm{D}} \\
\delta(m)=\left(\omega(m) \tau_{\mathrm{D}}\right)^{1 / 2}
\end{gathered}
$$

The $\omega(m)$ are the high-field $\mathrm{S} / \mathrm{T}_{0}$ transition frequencies of radical pairs with nuclear spin states $\{m\}$.

Mints and Pukhov ${ }^{727}$ have also solved the continuous diffusion SLE under high-field conditions but including also relaxational coupling to $T_{+}$and $T_{+}$. Neglecting exchange interaction and assuming singlet recombination at $r=r_{0}$ (radiation boundary condition), they derived analytical expressions for the recombination yields ${ }^{\mathrm{S}} Y_{\mathrm{S}},{ }^{\mathrm{T}} Y_{\mathrm{S}}$, and ${ }^{\mathrm{F}} Y_{\mathrm{S}}$.

Series expansions have been applied to solve eq 197 for general fields. Whereas Evans et al. ${ }^{700}$ considered exchange interaction and recombination as small perturbations and applied an iterative procedure to improve solutions of $\bar{\rho}\left(r_{0}\right)$, Haberkorn ${ }^{682}$ expanded $\bar{\rho}\left(r_{0}\right)$ in powers of $q_{k l}$, which yields more general solutions, since no restriction is imposed on the size of $J_{0}$ and $k_{0}$. The solution is given to second order in $q_{k l}$.

Shuskin ${ }^{726}$ applied perturbation methods to obtain analytical expressions as solutions to the continuousdiffusion SLE, which included $r$-dependent exchange or Coulombic interaction in high magnetic fields, i.e., considering $\mathrm{S} / \mathrm{T}_{0}$ transitions only. His results for the case of exchange interaction compare favorably with the numerical results of Pedersen and Freed. ${ }^{713}$ Analytical approximations to the case of radical ion pair diffusion in a Coulombic potential have been obtained under limiting conditions of weak $(x \ll 1)$ and strong $(x \gg 1)$ magnetic interaction, ${ }^{726}$ where the parameter $x$ is defined by

$$
x=1 / 2 r_{\mathrm{c}}(\omega / 2 D)^{1 / 2}
$$

with the Onsager radius $r_{\mathrm{c}}$ given by

$$
r_{\mathrm{c}}=\frac{e_{0}^{2}}{4 \pi \epsilon_{0} k T}
$$

In a more general treatment of spin-dependent radical ion pair recombination, Shushin ${ }^{729}$ demonstrated that the effect of Coulombic attraction corresponds to exponential escape from a cage in the strong-magnetic-interaction case, whereas the free diffusion model is a good approximation in the weak-magnetic-interaction case.

\section{Time-Dependent Solutions of SLES}

Few authors have determined time-dependent solutions of radical pair recombination yields on the basis of direct solutions of SLEs.

Within the framework of the exponential SLE, Tang and Norris ${ }^{707}$ have calculated the time evolution of the transient radical pair state in photosynthetic reaction centers. They solved numerically the matrix equation for the Laplace transform of the spin density matrix $\hat{\rho}(s)$, from which $\rho(t)$ was obtained by numerically performing the inverse Laplace transformation.

Lendi ${ }^{705}$ in his perturbational treatment of a general exponential-type SLE provided also time-dependent solutions valid in the limit of short and long times. Time-dependent solutions on the basis of the continu- ous-diffusion SLE have been worked out by Werner et al. ${ }^{643}$ who applied numerical integration by finite-difference methods and by Haberkorn ${ }^{721}$ in a manner similar to his time-integrated solution method outlined above. His result is presented as a series expansion in the matrix elements $q_{k l}$ defined above and is valid in the so-called low-frequency limit and at times longer than $\tau_{\mathrm{D}}$.

The relatively modest interest in time-dependent theoretical treatments may be due to the fact that, until recently, only few time-resolved measurements in the nanosecond region have been available for MFEs on reaction yields. An exception to this is the situation in radioluminescence. Here, however, there is in general no spin-dependent recombination rate, and hence time-dependent MFEs can be calculated with the assembling formalism of independent spin motion and diffusion, described in section 5 .

There is now an increasing amount of work dealing with time-resolved magnetic field effects in photosynthetic reaction centers and in micellar solutions. For a proper understanding of these experiments more work on time-dependent theories, which in the latter case should also include paramagnetic relaxation effects, would be desirable.

\section{Triplet-Triplet and Triplet-Doublet Pairs}

The main difference of theoretical treatments dealing with TT and TD pairs as compared to DD pairs results from the dominant role the zero-field splitting (ZFS) plays in triplets. Thus, in general, the influence of nuclear spin states is even neglected in such problems, so that the dimension of the pair spin space is at most 9 , limiting the computational effort when aiming for exact solutions.

A general treatment of TT and TD pair behavior in a magnetic field will start with a SLE including similar terms as eq 120 used for radical pairs.

With the exception of singlet fission, TT and TD pairs are generated by random encounters, corresponding to the F-pair situation with radicals. In this case a source term is included in the SLE accounting for the production of TT and TD pairs.

In the case of triplet fusion and triplet quenching by triplets, the source term is usually assumed in the form $^{73}$

$$
\begin{gathered}
G_{\mathrm{TT}}=1 / 9 k n_{\mathrm{T}}^{2} I \delta\left(r-r_{0}\right) \\
G_{\mathrm{TD}}=1 / 6 k n_{\mathrm{T}} n_{\mathrm{D}} I \delta\left(r-r_{0}\right)
\end{gathered}
$$

Here $n_{\mathrm{T}}$ and $n_{\mathrm{D}}$ are the bulk concentrations of triplets and doublets.

In the case of singlet fission the corresponding source term would be ${ }^{13}$

$$
G_{\mathrm{SF}}=k_{\mathrm{S}} Q_{\mathrm{S}} \delta\left(r-r_{0}\right)
$$

The reaction operator $\boldsymbol{K}$ will have to account for spin-selective reaction into the singlet, triplet, or quintet channel (for TT pairs) or the doublet or quartet channel (for TD pairs). For the TT case it may be written as ${ }^{736}$

$$
\boldsymbol{K} \rho=-1 / 2\left[\left(\lambda_{\mathrm{S}} Q_{\mathrm{S}}+\lambda_{\mathrm{T}} Q_{\mathrm{T}}+\lambda_{\mathrm{Q}} Q_{\mathrm{Q}}\right), \rho\right]_{+}
$$

The experimental quantities of interest are related to stationary flow rates $r_{\mathrm{S}}$ and $r_{\mathrm{D}}$ into the singlet channel 
TABLE 21. Literature Survey on Theories of Magnetokinetic Effects in TT and TD Pairs

\begin{tabular}{|c|c|c|c|c|c|c|c|c|}
\hline year & ref & pair type & medium $^{c}$ & $\begin{array}{l}\text { Merrifield } \\
\text { model }\end{array}$ & $\begin{array}{l}\text { spin } \\
\text { motion }\end{array}$ & $\mathrm{SLE}^{c}$ & $\begin{array}{l}\text { exchange } \\
\text { interaction }\end{array}$ & remarks \\
\hline \multirow[t]{2}{*}{1968} & Merrifield $^{71}$ & $\mathrm{~T} T$ & $\mathrm{C}$ & $x$ & & & & $b$ \\
\hline & Ern and Merrifield ${ }^{285}$ & TD & $\mathrm{C}$ & $x$ & & & & \\
\hline \multirow[t]{2}{*}{1970} & Johnson and Merrifield ${ }^{73}$ & TT & $\mathrm{C}$ & $g$ & & $\mathbf{E}$ & & $d$ \\
\hline & Suna $a^{456}$ & TT & $\mathrm{C}$ & & & $\mathrm{D}^{e}$ & & $f$ \\
\hline \multirow[t]{4}{*}{1971} & Closs $^{733}$ & TT & $\mathrm{s}$ & & $x^{h}$ & & $x$ & $i$ \\
\hline & Kearns and Stone ${ }^{366}$ & $\mathrm{TT}, \mathrm{TD}$ & S & $x^{j}$ & & & $x$ & $m$ \\
\hline & Avakian and Suna $a^{734}$ & $\mathrm{TT}, \mathrm{TD}$ & $\mathrm{C}$ & & $x^{k}$ & & & \\
\hline & Avakian et al.360 & $\mathrm{TT}$ & $S$ & & & $\mathrm{E}^{l}$ & & \\
\hline \multirow[t]{2}{*}{1972} & Kubarev et al. ${ }^{735}$ & TT & I & $x$ & & & & $m$ \\
\hline & Geacintov and Swenberg 367 & $\mathrm{TT}$ & $\mathrm{S}$ & $x$ & & & $x$ & $m$ \\
\hline \multirow[t]{2}{*}{1974} & Bouchriha et al. ${ }^{235}$ & $\mathrm{TD}$ & $\mathrm{C}$ & $g$ & & $\mathrm{E}$ & & $d$ \\
\hline & Tachikawa and Bard ${ }^{369}$ & $\mathrm{TT}, \mathrm{TD}$ & $\mathrm{S}$ & $x^{h}$ & & & & \\
\hline 1975 & Atkins and Evans ${ }^{338}$ & $\mathrm{TT}, \mathrm{TD}$ & $S$ & & $x^{0}$ & & $x$ & 0 \\
\hline $1976 / 1977$ & Lendi et al. ${ }^{69,770}$ & $\mathrm{TT}$ & $\mathrm{S}$ & & & $\mathrm{E}^{i}$ & $x$ & $p$ \\
\hline 1978 & Lesin $^{70}$ & TT, TD & $\mathrm{C}$ & $x^{q}$ & & & & \\
\hline 1979 & Altwegg $^{771}$ & $\mathrm{TT}$ & $\mathrm{C}$ & & & $\mathrm{D}^{r}$ & & \\
\hline 1983 & Vankan and Veeman ${ }^{288}$ & $\mathrm{TT}$ & $\mathrm{C}$ & $x^{s}$ & & & & \\
\hline 1984 & Schulten ${ }^{674}$ & $\mathrm{TT}, \mathrm{TD}$ & $\mathrm{C}, \mathrm{S}^{t}$ & & $x^{u}$ & & & $u$ \\
\hline \multicolumn{9}{|c|}{$\begin{array}{l}{ }^{a} \mathrm{C} \text {, crystal; S, solution; I, interface or surface. }{ }^{b} \text { Zero-field and high-field situation, anticipation of high-field resonances. }{ }^{c} \mathrm{E} \text {, exponential } \\
\text { ype; D, diffusional type SLE. }{ }^{d} \text { Derivation of high-field resonance line shape. }{ }^{e} \text { Spin relaxation included. } f \text { Two-dimensional diffusion. } \\
\text { Derived as limiting case. }{ }^{h} \text { Transitions between pair spin states'assumed to be due to hfc. }{ }^{i} \text { Discussion of potential CIDNP in triplet } \\
\text { carbene dimerization. }{ }^{j} \text { Independent suggestion. }{ }^{k} \text { Description in terms of coherent spin motion. 'Averaging over static angular distribution } \\
\text { cf pairs. }{ }^{m} \text { Case of triplet quenching by oxygen. }{ }^{n} \text { Estimation of high-field effect. }{ }^{o} \text { First dynamical treatment, taking molecular motion in } \\
\text { solution into account. }{ }^{p} \text { Complete field dependence for various pair parameters. }{ }^{q} \text { Analytical expression for field dependence in the direction } \\
\text { flow-field resonances. }{ }^{m} \text { Taking into account anisotropy of spin relaxation. }{ }^{s} \text { Effect due to Boltzmann distribution under high-field, low- } \\
\text { cemperature conditions. }{ }^{t} \text { Dynamical and statistical case of molecular motion. }{ }^{u} \text { Only spin motion, no recombination yields evaluated. }\end{array}$} \\
\hline
\end{tabular}

(in the case of delayed fluorescence) or the doublet channel (in the case of triplet quenching by doublets or charge-carrier detrapping). The stationary rates $r_{\mathrm{S}}$ or $r_{\mathrm{D}}$ may be used to define effective reaction probabilities $\gamma$ for triplet-triplet or triplet-doublet encounters:

$$
\begin{gathered}
r_{\mathrm{S}}=\gamma_{\mathrm{TT}} k n_{\mathrm{T}}{ }^{2} \\
r_{\mathrm{D}}=\gamma_{\mathrm{TD}} k n_{\mathrm{T}} n_{\mathrm{D}}
\end{gathered}
$$

\section{TT and TD Reactions in Molecular Crystals}

Since magnetic-field-dependent triplet-triplet and singlet-doublet interactions were first detected in molecular crystals, theoretical treatments, too, were first developed for this situation. The current theory had been rather completely worked out by 1973 and has been explained in several excellent reviews. ${ }^{1,12,13,16,25}$ Hence we shall confine ourselves to a presentation of a survey of original contributions, which are collected in Table 21 together with theoretical work on solution reactions, and give a few remarks on the essential aspects of the treatments.

According to Johnson and Merrifield, ${ }^{73}$ the triplettriplet annihilation, as represented by the reaction scheme

$$
\mathrm{T}+\mathrm{T} \underset{k_{-1}}{\stackrel{k_{1}}{\rightleftarrows}}(\mathrm{TT}) \stackrel{k_{2}}{\longrightarrow} \mathrm{S}^{*}+\mathrm{S}_{0}
$$

is described by a SLE of the (TT)-pair spin density matrix:

$\dot{\rho}(t)=-i[H, \rho]_{-}-k_{-1} \rho-1 / 2 k_{2}\left[Q_{\mathrm{S}}, \rho\right]_{+}+1 / 9 k_{\mathrm{i}} n_{\mathrm{T}}{ }^{2} I$

which is the analogue of the exponential-type SLE (eq 167) of the radical pair mechanism including, however, a source term for production of new (TT) pairs from triplet encounters in the volume. The density matrix $\rho$ of the pair is assumed independent of pair separation $r$, since the triplet pair is considered at a fixed distance and the stochastic operator $\Gamma(\mathbf{r}, \Omega)$ of eq 120 is reduced to a monoexponential dissociation rate constant $k_{-1}$. The rotational degree of freedom $\Omega$ is also omitted, since in the crystal all the triplet excitons have the same orientation and cannot rotate. The spin Hamiltonian $H$ is usually taken as a simple superposition of the individual triplet spin Hamiltonians, neglecting any exchange interaction.

$$
\begin{aligned}
& H=H_{\mathrm{T}(1)}+H_{\mathrm{T}(2)} \\
& H_{\mathrm{T}(i)}=D_{i}\left(S_{z, i}{ }^{2}-1 / 3 \boldsymbol{S}_{i}{ }^{2}\right)+E\left(S_{x, i}{ }^{2}-S_{y, i}{ }^{2}\right)+g \mu_{\mathrm{B}} \mathbf{B}_{0} \boldsymbol{S}_{i}
\end{aligned}
$$

Thus the energy eigenfunctions of the pair are just the products of the energy eigenfunctions of the individual triplets. They are, however, in general not eigenfunctions of the total electron spin of the pair, which may correspond to a singlet, a triplet, or a quintet total spin state. If $H_{\mathrm{T}(1)}$ and $H_{\mathrm{T}(2)}$ are identical, the parity of the pair spin functions is different for triplet pair states (odd) and singlet and quintet pair states (even, with respect to exciton exchange). This precludes singlettriplet or triplet-quintet mixing in the respective eigenfunctions.

Under stationary conditions and when the bulk concentration $n_{\mathrm{T}}$ of triplet excitons is independent of the triplet-triplet annihilation process, setting $d \rho / d t=0$ yields a linear matrix equation for $\rho$. In the general case it is solved numerically. Simple analytical solutions may be obtained, however, under the condition

$$
E_{m}-E_{n} \gg k_{-1}, k_{2}
$$

( $E_{n}$ and $E_{m}$ are any two eigenvalues of $H$ ), i.e., if there are no near degeneracies of (TT)-pair energy eigenstates as compared to their width. The following result is obtained for $\gamma_{\mathrm{TT}}$ :

$$
\gamma_{\mathrm{TT}}=\frac{1}{9} k_{1} \sum_{i} \frac{k_{2}\left|S_{i}\right|^{2}}{k_{-1}+k_{2}\left|S_{i}\right|^{2}}
$$


which was originally suggested by Merrifield on intuitive arguments. ${ }^{71}$ It corresponds to a superposition of independently decaying energy eigenstates with a specific annihilation rate constant of $k_{2}\left|S_{i}\right|^{2}$, proportional to their singlet character (cf. section II).

The coefficients $S_{i}$ depend on the magnetic field strength and direction. Whereas at zero field $\left|S_{i}\right|^{2}$ is distributed over three eigenstates (if we deal with triplet pairs with parallel orientation of the ZFS tensor axes), it will be mixed into more energy eigenstates as the magnetic field increases, as long as $g \mu_{\mathrm{B}} B_{0}$ does not greatly exceed the zero-field splitting. Due to the orientational dependence of the $\left|S_{i}\right|^{2}$ coefficients in this field region, the so-called low-field resonances may be observed as the crystals are rotated in a constant magnetic field. Such resonance orientations are encountered where $B_{0}$ is parallel to either of $x^{*}, y^{*}$, or $z^{*}$, the main axes of the triplet exciton ZFS tensor. Compact analytical expressions for the field dependence of $\gamma_{\text {TT }}$ with $B_{0}$ along such low-field resonance directions have been derived by Lesin. ${ }^{70}$

Kubarev et al. ${ }^{735}$ investigated the situation of tetracene/oxygen triplet pairs with a specific orientation of the oxygen molecules. Using the Merrifield formalism, they calculated the MFE on the rate constant of singlet oxygen production. They obtained several orientational resonances for magnetic fields where the Zeeman energy is in the order of magnitude of ${ }^{3} \mathrm{O}_{2}$ ZFS.

When $g \mu_{\mathrm{B}} B_{0}$ largely exceeds the ZFS, the (TT)-pair singlet character is concentrated on fewer energy eigenstates than in zero field, so that the triplet-triplet annihilation rate constant drops below the zero-field value. At very high fields the singlet pair state is distributed over two energy eigenstates only, with $\left|S_{i}\right|^{2}=$ $1 / 3$ and $\left|S_{2}\right|^{2}=2 / 3$. Then the limiting value of $\gamma_{\text {TT }}$ is

$$
\gamma_{\mathrm{TT}}\left(B_{0} \rightarrow \infty\right)=\frac{1}{9} k_{1}\left(\frac{1 / 3 k_{2}}{k_{-1}+k_{2} / 3}+\frac{2 / 3 k_{2}}{k_{-1}+2 k_{2} / 3}\right)
$$

In general, with solution of the spin-Hamiltonian eigenvalue problem, eq 216 has served to obtain reasonable quantitative fits of the magnetic field strength and orientational dependence of delayed fluorescence. ${ }^{73}$ The two high-field levels, which carry all the singlet character, become degenerate at certain directions of the magnetic field (cf. Figure 11). A level crossing occurs, which causes the so-called high-field resonances. The line shape of these may be obtained from the SLE for a reduced $2 \times 2$ density matrix, with the result ${ }^{73}$

$$
\gamma_{\mathrm{TT}}^{\mathrm{HF}}(\omega)=\gamma(\infty)-\frac{\gamma(\infty)-\gamma^{\prime}(\infty)}{1+\omega^{2} / \Delta^{2}}
$$

with

$$
\begin{gathered}
\omega=\left(E_{1}-E_{2}\right) / k_{-1} \\
\gamma^{\prime}(\infty)=\frac{1}{9} k_{1} \frac{k_{2}}{k_{-1}+k_{2}} \\
\Delta^{2}=\frac{\left(k_{-1}+k_{2}\right)\left(k_{-1}+k_{2} / 2\right)^{2}}{k_{-1}\left(k_{-1}+k_{2} / 3\right)\left(k_{-1}+2 k_{2} / 3\right)}
\end{gathered}
$$

At exact resonance $\gamma_{\mathrm{TT}}{ }^{\mathrm{HF}}(0)=\gamma^{\prime}(\infty)$; i.e., the situation corresponds to a concentration of singlet character on one energy eigenstate only. From the line width $\Delta$ of the high-field resonances the ratio $k_{2} / k_{-1}$ can be determined. In order to fix the absolute values of these rate constants, fitting to the complete MFD is required. The result thus obtained for anthracene $e^{73}$ was $k_{-1}=2.8$ $\times 10^{9} \mathrm{~s}^{-1}$ and $k_{2}=1.1 \times 10^{9} \mathrm{~s}^{-1}$.

The formalism described so far for TT pairs may be applied in an analogous way to TD pairs, too. Corresponding formulas have been derived by Bouchriha et al. ${ }^{235}$ Since the exponential SLE approach of Johnson and Merrifield ${ }^{73}$ corresponds to a kinetic description of the TT annihilation process in terms of rate constants (scheme 211), which may be an oversimplified model of physical reality, Sun ${ }^{456}$ studied the kinematics of triplet exciton annihilation in molecular crystals on the basis of the full $r$-dependent SLE analogue of eq 120 . The stochastic motion $(\Gamma(\mathbf{r}))$ of the TT pair was represented either by a continuous differential operator or by a difference operator, modeling the hopping-type diffusion of excitons in a crystal. Restricting the reactivity operator $\boldsymbol{K}(r)$ to nearest-neighbor interactions, Suna obtained analytical solutions for the spin-independent case with one, two, and three dimensions of diffusion. He showed clearly that only in the threedimensional case is there a unique definition of a stationary diffusion-controlled recombination rate constant. In this case a certain isomorphism between diffusional and kinetic (exponential) models exists, whereby the following relation holds true:

$$
k_{2} / k_{-1}=\lambda v / k_{\text {diff }}
$$

Here $k_{\text {diff }}$ is the diffusion-controlled bimolecular recombination rate constant and $\lambda$ is a first-order reaction rate constant, which is assumed to be uniform within the reaction volume $v$. In one or two dimensions such a relation does not generally exist because there will be complete recombination even for very small $\lambda$, since in these reduced dimensions there is an infinite number of reencounters of the pair. Only if a unimolecular decay of the triplet excitons determines their bulk concentration can effective values of a diffusion-controlled reaction rate constant and the ratio $k_{2} / k_{-1}$ be unambiguously defined.

The spin-dependent problem was solved by using the continuous-diffusion form of $\Gamma(\mathbf{r})$ and restricting $H(\mathbf{r})$ and $\boldsymbol{K}(r)$ to nearest-neighbor interactions. The Green's function technique was applied to transform the continuous-diffusion SLE to a linear matrix equation. With this procedure Suna anticipated the techniques later used by Evans et al..$^{700}$ and Haberkorn ${ }^{682}$ for the treatment of the radical pair mechanism with $\delta$-functional type exchange and reactivity terms. Furthermore, Suna incorporated the effect of spin relaxation using, however, one relaxation parameter only.

This form of the theory was applied to analyze the experimental results on TT annihilation in anthracene crystals. This system actually represents a case of a two-dimensional exciton diffusion in the crystallographic $a b$ plane with relatively slow hopping in the $c$ direction between the planes. The hopping rate between the planes could be treated as a monomolecular exciton decay whereas the diffusion in the plane provided a model for the reencounter kinematics of the TT pairs. The relation to the Johnson and Merrifield parameters $k_{2}$ and $k_{-1}$ was established and related to 
physically more meaningful quantities (cf. also the review by Swenberg and Geacintov ${ }^{13}$ ).

\section{2. $T T$ and TD Pair Reactions in Solution}

The situation in solution is characterized by the fact that random molecular orientations with respect to the magnetic field and with respect to the second moiety in the pair occur. Strictly speaking, the orientational coordinates $\Omega$ should be even treated dynamically (vide infra).

Unless there is an appreciable energy gap between pair states of different multiplicities, it may be shown by general arguments that in zero field the singlet (doublet) probabilities $\left|S_{i}\right|^{2}\left(\left|D_{i}\right|^{2}\right)$ are most uniformly distributed over the energy eigenstates $\left(\left\langle\left|S_{i}\right|^{2}\right\rangle_{\mathrm{av}}=1 / 9\right.$ in TT pairs and $\left\langle\left|D_{i}\right|^{2}\right\rangle_{\mathrm{av}}=1 / 6$ in TD pairs) while the concentration of singlet (doublet) character is highest in the high-field case $\left(\left\langle\left|S_{i}\right|^{2}\right\rangle_{\mathrm{av}}=1 / 2\right.$ for 2 out of $9 \mathrm{ei}$ genstates in TT pairs and $\left\langle\left|D_{i}\right|^{2}\right\rangle_{\mathrm{av}}=1 /{ }_{4}$ for 4 out of 6 eigenstates in TD pairs). From these limiting values, and using the Merrifield formula (eq 216) or its analogue for TD pairs, the $k_{2} / k_{-1}$ ratio can be obtained from the saturation effect at high fields. This method was applied by Tachikawa and Bard, ${ }^{369}$ who thus evaluated $k_{2} / k_{-1}=0.1$ for anthracene TT annihilation in solution and a corresponding value of 0.44 for anthracene triplet quenching by TMPDA radical cations (cf., however, below).

An energetic separation of TT pair states of different multiplicity may arise from intermolecular spin-spin interaction and especially from charge-transfer interactions. These have been considered in some detail for triplet pairs, including ${ }^{3} \mathrm{O}_{2}$ by Kearns and Stone ${ }^{366}$ and by Geacintov and Swenberg. ${ }^{367}$ If the quintet/singlet or triplet/singlet splittings exceed the ZFS of the individual triplets, the singlet character will not be dispersed over the energy eigenstates in zero field. At higher fields level crossings of the type $Q / S$ and $T / S$ will occur, giving rise to a distribution of pair singlet character in the crossing region. Actually, this is an anticrossing phenomenon (cf. Figure 49), and according to the Merrifield formula, the annihilation rate will increase in such regions. Kearns and Stone and Geacintov and Swenberg have qualitatively discussed the implication of the exchange energy terms $J_{\mathrm{QS}}$ and $J_{\mathrm{TS}}$ on the rate of triplet quenching by oxygen. They disagree on the role of singlet-triplet mixing in (TT)-pair spin states by the operator $H_{\mathrm{D}}$. One should note that matrix elements of the form $\left.{ }^{3}(\mathrm{TT})\left|H_{\mathrm{D}}\right|^{1}(\mathrm{TT})\right\rangle$ are zero ${ }^{338,736}$ due to the Wigner-Eckart theorem.

More quantitative treatments of TT annihilation in solution accounting for the angular freedom of molecular motion by averaging the results of the exponential-type SLE over a quasi-static angular distribution of T'T pairs have been reported by Avakian et al..$^{360}$ and by Lendi et al. ${ }^{69,770}$

Lendi et al. ${ }^{70}$ used the numerical solution of the full $9 \times 9$ density matrix equation for TT annihilation leading to excimer emission of pyrene. They assumed a fixed sandwich pair configuration and performed the average over the polar angle between the principle $\mathbf{D}$ tensor axis and the magnetic field. Using suitable parameters, they obtained a good fit of the experimental results for a range of temperatures.

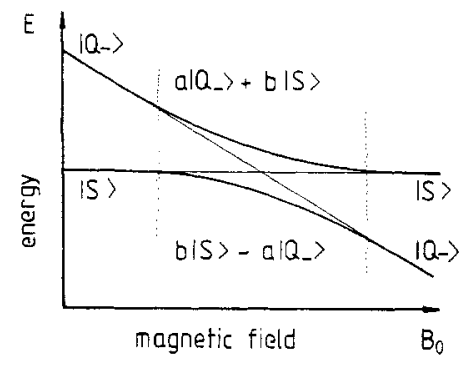

Figure 49. Zeeman anticrossing of singlet and quintet pair states of the TT pair.

In the low-viscosity range where $k_{-1}$, the diffusive separation rate constant of a TT pair, is fairly large, a perturbational treatment of the SLE problem may be applied which yields analytical solutions. This method was used by Avakian et al. ${ }^{360}$ In such a perturbation treatment the spin eigenstates of the pair are used as the unperturbed basis whereas the traceless $H_{\mathrm{D}}$ is considered as a perturbation. The solution is given to second order, and angular averaging is easily performed. Applying this treatment to the anthracene TT-annihilation problem, Avakian et al ${ }^{360}$ determined the rate parameters $k_{2}=1.6 \times 10^{11} \mathrm{~s}^{-1}$ and $k_{-1}=1.3 \times 10^{10} \mathrm{~s}^{-1}$, yielding a ratio of $k_{2} / k_{-1}=12$, which is much larger than the value obtained by Tachikawa and Bard on the basis of limiting cases of the Merrifield formula.

The perturbation treatment has been also applied by Lendi et al. ${ }^{69}$ Here the criterion of applicability of the perturbation treatment was derived as

$$
k_{-1}>8 / 3 D
$$

Furthermore, the effect of exchange energy $\left(J_{\mathrm{QS}}\right)$, splitting singlet and quintet pair states, was included. To second order the result for the efficiency parameter of TT annihilation is given as

$$
\begin{aligned}
& \gamma_{\text {TT }}= \\
& \frac{\lambda_{\mathrm{S}}}{9\left(\lambda_{\mathrm{S}}+k_{-1}\right)}\left(1+\frac{16\left(\lambda_{\mathrm{S}}-\lambda_{\mathrm{Q}}\right)\left(\lambda_{\mathrm{S}}+\lambda_{\mathrm{Q}}+2 k_{-1}\right)}{45\left(\lambda_{\mathrm{S}}+k_{-1}\right)\left(\lambda_{\mathrm{Q}}+k_{-1}\right)} \times\right. \\
& \left.D^{* 2 \sum_{j=5}^{9}\left\{\left(\lambda_{\mathrm{S}}+\lambda_{\mathrm{Q}}+2 k_{-1}\right)^{2}+4\left[J_{\mathrm{QS}}+(7-j) g \mu_{\mathrm{B}} B_{0}\right]^{2}\right\}^{-1}}\right)
\end{aligned}
$$

with

$$
D^{* 2}=D^{2}+3 E^{2}
$$

The parameters $\lambda_{\mathrm{S}}, \lambda_{\mathrm{T}}$, and $\lambda_{Q}$ denote the rate constants of TT pair annihilation in the respective pair spin states. A maximum in the MFD of $\gamma_{\mathrm{TT}}$ due to Q/S level anticrossing is predicted under the following condition:

$$
\sqrt{3} J_{Q S}>k_{-1}+\left(\lambda_{S}+\lambda_{Q}\right) / 2
$$

Using eq 222 to fit the MFD of the pyrene monomer delayed fluorescence in 2-methyltetrahydrofuran at 18 ${ }^{\circ} \mathrm{C}^{363}$ yielded the values ${ }^{69} k_{-1}=2.4 \times 10^{10} \mathrm{~s}^{-1}, \lambda_{\mathrm{S}}=9 \times$ $10^{10} \mathrm{~s}^{-1}, \lambda_{\mathrm{Q}}=2 \times 10^{9} \mathrm{~s}^{-1}$, and $J_{\mathrm{QS}}=2 \times 10^{9} \mathrm{~s}^{-1}$, which are similar to Avakians et al.' ${ }^{360}$ results on anthracene TT annihilation as far as $k_{-1}$ and $\lambda_{\mathrm{S}} \equiv k_{2}$ are concerned. It is of interest that, in order to obtain an acceptable fit, a nonvanishing $\lambda_{Q}$ had to be assumed, indicating the activity of some quintet channel in $\mathrm{TT}$ annihilation of pyrene.

In the Merrifield model TT and TD pairs are considered from the point of view of independently de- 
caying, quasi-stationary eigenstates of the spin Hamiltonian. In the radical pair models, on the other hand, the usual picture is that of eigenstates of the pair's total electron spin, which are coherently converted into each other, due to individually different contributions of the two radicals to the spin Hamiltonian. This view is appropriate since the kinetically caused width of the spin levels normally exceeds their energy separation induced by the difference of single radical spin Hamiltonians. The justification of the Merrifield model, on the other hand, is that, due to the relatively large ZFS in molecular triplets, the energy separation of the pair eigenstates is normally (in crystals) larger than their kinetic width. In solutions, however, where the molecular constituents of the TT pair may undergo rapid rotational tumbling, the ZFS is averaged out, exhibiting its influence only by inducing spin relaxation between eigenstates of the spin Hamiltonian, their average energy being no longer determined by the ZFS parameters. Thus it appears that in liquid solutions the TT and TD pair recombination processes should be described in terms of spin-evolution and reencounter statistics, as in the radical pair models.

The concept of spin motion in TT and TD pairs has been used qualitatively by Avakian and Suna ${ }^{734}$ in order to give a first explanation of magnetic-field-dependent luminescence in crystals. It has been also invoked by Closs $^{733}$ in discussing the problem of CIDNP in the dimerization of trimethylenemethane derivatives, which are known to have triplet ground states. These authors, however, did not consider the interference between the effect of ZFS and molecular tumbling.

Only Atkins and Evans ${ }^{338}$ made full use of this concept for quantitative calculation of the MFD of TT and TD reactions in solution. In their model they considered random recombination of TT and TD pairs, where the spin-selective reaction in the first encounter produces some overpopulation of quintet and triplet (case of TT pairs) or quartet (case of TD pairs) spin states of the pair. The spin evolution of the polarized pairs is then calculated on the basis of independent spin relaxation in the separated triplets and doublets, making quantitative use of the Redfield formalism. The following result is obtained for the time dependence of singlet character of a TT pair after a random encounter at $t=0$, when the singlet probability was set to zero by virtue of the TT annihilation process:

$$
\begin{aligned}
& \left\{T,\left.Q\right|_{p_{S}}(t)=\frac{1}{9}\left(1-\frac{1}{8}\left[\exp \left(-6 R_{1100} t\right)+2 \exp \left(2 R_{1-11-1} t\right)\right.\right.\right. \\
& \left.\left.+\exp \left(-2 t / T_{1}\right)+4 \exp \left(2 R_{1010} t\right) \cosh \left(2 R_{100-1} t\right)\right]\right)
\end{aligned}
$$

with

$$
\begin{gathered}
T_{1}^{-1}=R_{1100}+2 R_{11-1-1} \\
R_{1100}=-R_{100-1}=2 / 15 k\left(\omega_{\mathrm{T}}\right) \\
R_{11-1-1}=4 / 15 k\left(2 \omega_{\mathrm{T}}\right) \\
R_{1010}=-1 / 5\left\{k(0)+k\left(\omega_{\mathrm{T}}\right)+2 / 3 k\left(2 \omega_{\mathrm{T}}\right)\right\} \\
R_{1-11-1}=-2 / 15\left\{k\left(\omega_{\mathrm{T}}\right)+2 k\left(2 \omega_{\mathrm{T}}\right)\right\}
\end{gathered}
$$

$$
\begin{gathered}
k(\omega)=\frac{D^{2} \tau_{2}}{1+\omega^{2} \tau_{2}^{2}}+\frac{3 E \tau_{2}^{\prime}}{1+\omega^{2} \tau_{2}^{\prime 2}} \\
\tau_{2}=1 /\left(4 D_{\perp}+2 D_{\|}\right) \\
\tau_{2}^{\prime}=1 /\left(4 D_{\|}+2 D_{\perp}\right) \\
\omega_{\mathrm{T}}=g_{\mathrm{T}} \mu_{\mathrm{B}} B_{0}
\end{gathered}
$$

$D_{\|}$and $D_{\perp}$ are the components of the axially symmetric rotational diffusion tensor of the triplet molecules.

The total recombination probabilities are obtained by integrating the diffusional reencounter probability with the singlet (or doublet) probability of the pair. Analytical expressions are provided for the MFD dependence of delayed fluorescence intensity due to TT annihilation in the absence or presence of competing TD quenching.

The results were used to analyze the experimental MFD observed by Avakian et al..$^{734}$ for TT annihilation of anthracene, affording a rate constant of $k_{\mathrm{T}}=7 \times 10^{8}$ $\mathrm{s}^{-1}$ for the annihilation reaction of a ${ }^{1}(\mathrm{TT})$ pair. This corresponds to a reaction probability of 0.15 in a ${ }^{1}$ (TT) pair encounter. The result deviates significantly from that evaluated on the basis of the exponential model by Avakian et al. ${ }^{734}$ (vide supra). A major difference of the two theories is the origin of the MFD. Whereas in the quasi-static model of Avakian et al..$^{360}$ and Lendi et al. ${ }^{770}$ the strongest MFD occurs in the region of an approximate matching of ZFS and Zeeman energy, the MFD in the Atkins and Evans model results from the field dependence of the Redfield relaxation matrix elements, the most sensitive change of their MFD occurring when the Larmor frequency matches the orientational relaxation rate constant.

Schulten ${ }^{674}$ has extended his spin-correlation-tensor method to handle the spin-motion problem of TT and TD pairs also. The required spin probabilities after producing the pair in a mixture of spin states that is devoid of the particular multiplicity to be developed are given by the following compact expressions $(\mathrm{Qa}=$ quartet, $\mathrm{Qi}=$ quintet):

$$
\begin{gathered}
\text { DD: }{ }^{\mathrm{s}} p_{\mathrm{T}}=3 / 4-{ }_{1} U:_{2} U \\
\text { TD: }{ }^{\mathrm{Qa}} p_{\mathrm{D}}=1 / 4-1 / 9\left({ }_{1} U:{ }_{2} U\right) \\
\text { TT: }{ }^{\text {Qi,T }} p_{\mathrm{S}}=1 / 3-1 / 72\left({ }_{1} V::_{2} V\right)
\end{gathered}
$$

The probabilities of the required pair multiplicities are expressed by inner products of two spin-correlation tensors, which may be evaluated for each of the pair moieties separately. They are defined as follows:

$$
\begin{gathered}
U_{\alpha \beta}=\left\langle\operatorname{Tr}\left\{S_{\alpha}(t) S_{\beta}(0)\right\}\right\rangle \\
V_{\alpha \beta \gamma \delta}=\left\langle\operatorname{Tr}\left\{S_{\alpha}(t) S_{\beta}(t) S_{\gamma}(0) S_{\delta}(0)\right\}\right\rangle
\end{gathered}
$$

where the indices $\alpha, \beta, \gamma$, and $\delta$ are running over the spherical tensor compounds of the triplet's or radical's electronic spin operator. The time-dependent spin operators are defined in the Heisenberg picture, whereby the time dependence is due to hyperfine coupling in the case of doublets and due to the ZFS for triplets. The angular brackets \langle\rangle denote averaging over nuclear spin states of radicals or over orientations of triplets. Closed-form analytical solutions of eq $227 \mathrm{a}-\mathrm{c}$ 
are provided for the zero-field and the high-field cases. Angular averaging is performed over static ensembles or over dynamic ensembles in the limiting case where the rotational motion is fast with respect to the spin motion. Unfortunately, the author did not discuss the relation of his results to those of Atkins and Evans, ${ }^{338}$ which is not obvious.

\section{E. Reaction-Yleld-Detected Magnetic Resonance}

Although there are cases where microwave-induced effects on chemical reaction yields can be observed at zero field (cf., e.g., the method of zero-field ODMR in molecular triplet spectroscopy), in most cases the RYDMR effects observed represent microwave-induced modifications of effects from static magnetic fields on reaction yields. Generally speaking, the reaction yield may be expressed as a function $Y\left(B_{0}, B_{1}, \omega\right)$ and the various kinds of "magnetic spectra" (MARY, RYDMR, $B_{1}$ ) may be envisaged as appropriate projections of this function as has been demonstrated in a synoptic diagram by Lersch and Michel-Beyerle ${ }^{93}$ (cf. Figure 50).

The development of RYDMR theories has been closely related to experimental work going on in the field. Thus, mechanistically, the emphasis has been on reactions involving DD and TT pairs. Furthermore, for DD pairs all the treatments applied so far refer to the so-called "high-field" case; i.e., only $\mathrm{S} / \mathrm{T}_{0}$ mixing is considered as a microwave-independent ISC mechanism, and nuclear spin states are considered as stationary so that the total problem can be solved as a superposition of independent solutions for an ensemble of radical pairs with different nuclear spin states. A survey of original references, dealing theoretically with RYDMR effects, is given in Table 22.

\section{Kinetic Schemes with Quasi-Stationary Spin States}

The easiest situation to deal with theoretically is when the eigenstates of the time-independent part of the spin Hamiltonian are clearly separated with respect to their kinetic width. Then the effect of resonant microwaves may be described as inducing transitions between the quasi-stationary spin levels, and the overall kinetics may be treated by a set of coupled kinetic rate equations for the populations. This method, which corresponds to the simple Merrifield model in the case of nonresonant magnetic field effects, has been applied by Ruedin et al. ${ }^{736}$ for ODMR with F-center pairs (DD case) in alkali halide crystals and by Frankevich et al. ${ }^{106,544,547}$ for RYDMR observed by delayed and prompt fluorescence in organic molecular crystals (TT and TD case; for a review of this and other RYDMR work, cf. Frankevich and Kubarev ${ }^{41}$ ).

Within these approaches the line-width problem is handled by using the usual ESR line-width expression for the frequency dependence of the microwave-induced transition rate.

\section{Spin Motion in a Microwave Magnetic Field}

In liquid solutions it was logical to extend the concept of spin-motion-modified recombination kinetics (cf. section V.C.5) to include also the influence of a microwave field. Kubarev and Pschenichnov ${ }^{567}$ were the first

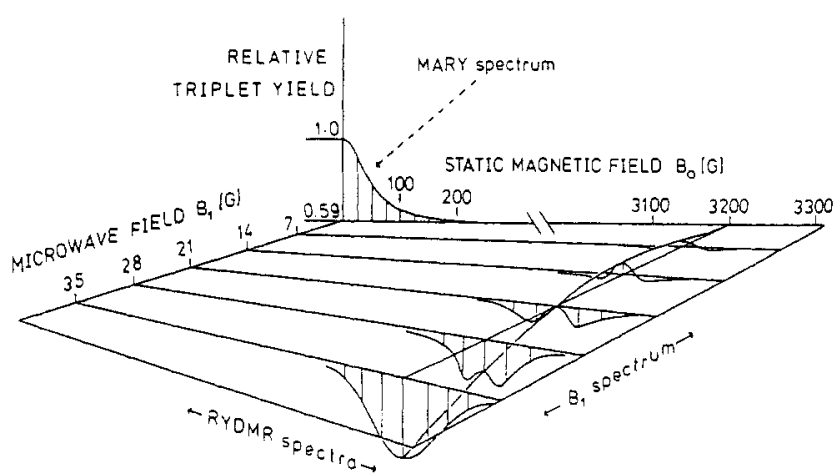

Figure 50. Synopsis of MARY spectrum, RYDMR spectrum, and the $B_{1}$ spectrum as detectable from the relative triplet yield of recombining radical pairs generated with singlet spin alignment. Reprinted from ref 93 with kind permission of M. E. MichelBeyerle; copyright 1983 Elsevier Science Publishers B.V.

to use this approach in order to estimate the $B_{1}$ strength necessary to detect RYDMR effects for radical pair recombination in solution.

Treatments of the RYDMR line shape based on a more general spin motion have been reported by $\mathrm{Ku}$ barev et al. ${ }^{737,738}$ and by Doktorov et al. ${ }^{711}$ Here, e.g., for a singlet-produced radical pair in a definite nuclear spin state, the spin motion under high-field static magnetic field conditions and at arbitrary $\mathrm{S} / \mathrm{T}_{0}$ coupling strength $\Delta\left(\mathrm{ST}_{0}\right)$ is given by ${ }^{737,738}$

${ }^{\mathrm{s}} p_{\mathrm{S}}=A+B \cos \left[\left(\Omega_{1}+\Omega_{2}\right) t\right]+C \cos \left[\left(\Omega_{1}-\Omega_{2}\right) t\right]+$
$D\left[\cos \left(\Omega_{1} t\right)+\cos \left(\Omega_{2} t\right)\right](229)$

with

$$
\Omega_{1,2}=\left[\left(\omega_{0} \pm \Delta\left(\mathrm{ST}_{0}\right) / 2-\omega\right)^{2}+\omega_{1}^{2}\right]^{1 / 2}
$$

where $\omega_{0}$ is the average Larmor frequency of both radicals in their respective nuclear spin states, $\omega$ is the microwave frequency, and $\omega_{1}$ the Rabi frequency, defined as

$$
\omega_{1}=g \mu_{\mathrm{B}} B_{1} / \hbar
$$

where $g$-factor differences can be usually neglected. The coefficients $A$ to $D$ are given by

$$
\begin{gathered}
A=B+C \\
B=1 / 2 \sin ^{4}\left[\left(\theta_{1}-\theta_{2}\right) / 2\right] \\
C=1 / 2 \cos ^{4}\left[\left(\theta_{1}-\theta_{2}\right) / 2\right] \\
D=1 / 4 \sin ^{2}\left[\theta_{1}-\theta_{2}\right]
\end{gathered}
$$

with

$$
\begin{gathered}
\sin \theta_{1,2}=\omega_{1} / \Omega_{1,2} \\
\cos \theta_{1,2}=\left(\omega_{0}+\Delta\left(\mathrm{ST}_{0}\right) / 2-\omega\right) / \Omega_{1,2}
\end{gathered}
$$

The radical pair recombination yield was calculated by integrating over an exponential decay function ${ }^{741}$ or using a reencounter probability function based on the continuous-diffusion equation. ${ }^{738}$ If $\omega_{1}, \tau^{-1} \ll \Delta\left(\mathrm{ST}_{0}\right)$ ( $\tau$ is the lifetime of the radical pair), each nuclear spin configuration gives rise to a doublet of RYDMR lines separated by $\Delta\left(\mathrm{ST}_{0}\right)$. Under such conditions and for an exponential decay of the radical pair with time constant $\tau_{0}$ the line width $\Delta \omega$ of each doublet components is given by

$$
\Delta \omega=\left(1 / \tau_{0}^{2}+\omega_{1}^{2}\right)^{1 / 2}
$$


TABLE 22. Survey of Theoretical Work on Reaction-Yield-Detected Magnetic Resonance (RYDMR)

\begin{tabular}{|c|c|c|c|c|c|c|c|}
\hline year & ref & pair type & $\begin{array}{c}\text { application } \\
\text { to }^{a}\end{array}$ & interactions $^{b}$ & $\begin{array}{l}\text { theoretical } \\
\text { method }^{c}\end{array}$ & $\begin{array}{c}\text { type of } \\
\text { solution }^{d}\end{array}$ & remarks \\
\hline 1972 & Ruedin et al. ${ }^{336}$ & DD & IC & SR & MES & $\mathrm{A}, \mathrm{L}$ & $e$ \\
\hline 1974 & Kubarev and Pshenichnov ${ }^{567}$ & DD & LS & $\Delta g$ & $\mathrm{SMI}^{\mu}$ & A, L & \\
\hline 1976 & Kubarev et al. ${ }^{737}$ & DD & LS & $\Delta g$ & SMIE/D & A, L & $f$ \\
\hline \multirow[t]{2}{*}{1977} & Frankevich et al. ${ }^{106}$ & TT & MC & ZFS & MEE & A, L & \\
\hline & Lesin et al. ${ }^{.52}$ & TT & MC & ZFS & $\mathrm{eSLE}{ }^{h}$ & A, L & $g$ \\
\hline \multirow[t]{2}{*}{1978} & Frankevich et al. ${ }^{544}$ & TT & MC & ZFS & MEE & A, L & \\
\hline & Frankevich et al. ${ }^{547}$ & TD & MC & ZFS & MEE & A, L & \\
\hline \multirow[t]{4}{*}{1980} & Haberkorn and Dietz ${ }^{574}$ & DD & $\mathrm{SC}$ & $\Delta g$ & eSLE, $H_{\text {eff }}$ & $A, L^{i}$ & \\
\hline & Kubarev et al. ${ }^{738}$ & DD & LS & $\Delta g, \mathrm{hfc}$ & SMID & $\vec{A}, \bar{L}$ & \\
\hline & von Schütz et al.90 & TT & $\mathrm{MC}$ & $\mathrm{ZFS}$ & MEE & A & $j$ \\
\hline & Movaghar et al. $.39,740$ & DD & $\mathrm{SC}$ & SR & MES & A & $k$ \\
\hline 1982 & Doktorov et al. ${ }^{741}$ & DD & LS, RL & hfc & SMIE & $A, L$ & $l$ \\
\hline $1982 / 1983$ & Lersch et al. ${ }^{93,742}$ & DD & $\mathrm{RC}$ & hfc, $J, D$ & eSLE, $H_{\text {off }}$ & N, L & $m, n$ \\
\hline 1983 & Tang and Norris ${ }^{743}$ & DD & RC & hfc, $J, D$ & eSLE, $H_{\text {eff }}$ & $\mathrm{N}, \overline{\mathrm{L}}$ & \\
\hline 1984 & Saik et al. ${ }^{99}$ & DD & LS, RL & hfc, SR & SMIE $^{\circ}$ & A, L & $p$ \\
\hline 1985 & Smirnov et al. ${ }^{557}$ & DD & LS, RL & hfe & $\mathrm{SMID}^{q}$ & $\mathrm{~N}$ & $r$ \\
\hline \multirow[t]{2}{*}{1987} & Hunter et al. ${ }^{744}$ & DD & $\mathrm{RC}$ & $\mathrm{hfc}^{,} J$ & eSLE, $H_{\text {eff }}$ & $\mathrm{A},{ }^{8} \mathrm{~L}$ & \\
\hline & Lersch and Michel-Beyerle ${ }^{745}$ & DD & $\mathrm{RC}$ & $\mathrm{hfc}^{,}{ }^{s} J$ & eSLE, $H_{\text {eff }}$ & $\mathrm{A},{ }^{8} \mathrm{~L}$ & $t$ \\
\hline
\end{tabular}

${ }^{a}$ IC, ionic crystals; LS, liquid solution; MC, molecular crystal; RC, photosynthetic reaction centers; RL, radioluminescence; SC, semiconductors. ${ }^{b} \mathrm{hfc}$, hyperfine coupling; $\Delta g$, difference of Zeeman interaction; $J$, exchange interaction; $D$, spin-spin dipolar interaction; SR, spin relaxation included as incoherent transition. ${ }^{c} H_{\text {eff }}$, effective spin Hamiltonian including imaginary kinetic terms; eSLE, exponential SLE; MEE (MES), master equation for pair energy (spin) eigenstate populations; SMIE (SMID), spin motion integrated with exponential (diffusional) reactivity function $f(t) .{ }^{d} \mathrm{~A}$, analytical; $\mathrm{N}$, numerical RYDMR line shape $(\mathrm{L})$. ${ }^{e}$ Luminescence from pairs of $\mathrm{F}$ centers. ${ }^{f} \mathrm{Explicit}$ formula of spin motion for S, T, and F pairs. ${ }^{8}$ Study of level-crossing phenomenon. ${ }^{h}$ Time-dependent series expansion. ${ }^{i}$ Special case of well-separated lines. ${ }^{j}$ Zero-field case; special attention to MW power dependence. ${ }^{k}$ Spin-dependent effects on luminescence and photoconductivity. 'Also analysis of delay-narrowing effect in time-selected observation. ${ }^{m}$ Critical comparison of RYDMR and MARY method. ${ }^{n}$ Also RYDMR anisotropy. ${ }^{\circ}$ Also time integration with $f \propto t^{-3 / 2}$. $P$ Including exchange narrowing by electron hopping. 9 Diffusion in Coulombic potential. ${ }^{r}$ Dependence of RYDMR intensity on diffusion parameters. ${ }^{s} \mathrm{hfc}$ treated as perturbation. ${ }^{t}$ Analytical conditions for line maxima and minima. "Case of $\Delta g B_{0} \gg g B_{1}$, coherent MW transitions, recombination yield averaged over one period.

When the microwave power increases and $\omega_{1}$ approaches $\Delta\left(\mathrm{ST}_{0}\right)$, the RYDMR spectrum changes as shown in Figure 51. The lines start to broaden and to merge with each other. However, before this happens a line inversion starts from the center and continues until finally the total line is inverted. As $\omega_{1}$ is still increased further the inverted line continues to broaden. This behavior may be rationalized with the level scheme depicted in Figure 51. Here the energy of the electronic spin states of the radical pair, measured in a system of reference that rotates around the $B_{0}$ axis with the microwave frequency $\omega$, is plotted against $\omega$. The spin states $\mathrm{S}$ and $\mathrm{T}_{0}$ are coupled to two new eigenstates $\left(\mathrm{ST}_{0}\right)_{1}$ and $\left(\mathrm{ST}_{0}\right)_{2}$, split by the energy gap $\Delta\left(\mathrm{ST}_{0}\right)$. Several cases of the microwave strength (corresponding to curves a-d) are indicated by vertical bars of length $\omega_{1}$. As the resonance frequencies $\omega_{0} \pm \Delta\left(\mathrm{ST}_{0}\right) / 2$ are passed, the microwave couples $\left(\mathrm{ST}_{1}\right)$ and $\left(\mathrm{ST}_{2}\right)$ to $\mathrm{T}_{+}$ and $\mathrm{T}_{-}$, respectively, wich means more efficient ISC and a spin of the RYDMR effect that is opposite to the MARY effect. The $\omega$ regions of resonance will become broader as $\omega_{1}$ increases (bars $\mathrm{b}$ and c). As $\omega_{1}$ becomes larger than $\Delta\left(\mathrm{ST}_{0}\right)$, however, at resonance all spin states simultaneously experience the influence of the microwave field $B_{1}$. Now $\mathrm{T}_{0}$ becomes distributed over two triplet components that are quantized parallel and antiparallel to $B_{1}$ and split away from $\mathrm{S}$ by an energy $\pm \hbar \omega_{1}$. Thus the $\mathrm{S} / \mathrm{T}_{0}$ coupling becomes less efficient and the $\mathbf{S} / \mathbf{T}_{0}$ ISC process ceases to take place. The effect causes an inversion of the RYDMR signal.

More recently, the method of integrating spin motion with a recombination probability function has been applied by Saik et al., ${ }^{99}$ using exponential radical pair decay, in order to study the effects of electron or hole hopping between like donor and acceptor molecules, and by Smirnov et al., ${ }^{557}$ with diffusion-controlled recombination in a Coulomb potential, for investigating
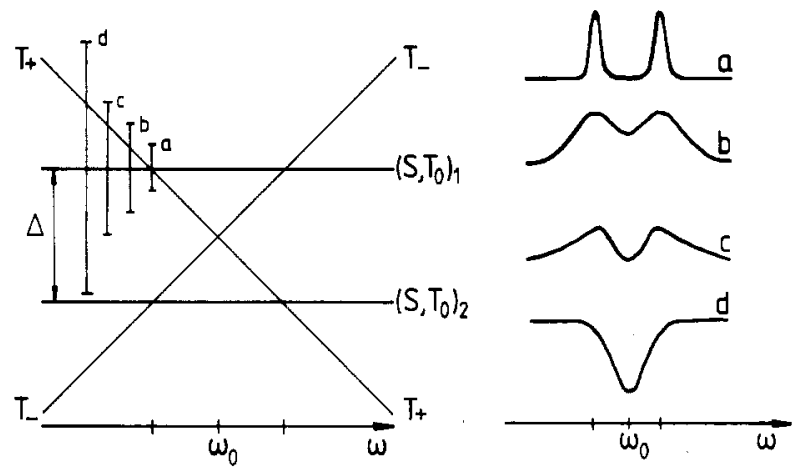

Figure 51. Schematic representation of RYDMR line-shape dependence of radical pairs on the ratio of microwave power (various cases of $\omega_{1}$ values are indicated by a-d and $S-\mathrm{T}_{0}$ coupling energy $(\Delta)$. Left, energy scheme in rotating frame; right, typical line shapes (adapted from ref 741). For details, cf. text.

the dependence of RYDMR intensity on diffusional parameters and spin-lattice relaxation. In the latter work numerical methods were applied to solve the problem of diffusion in a Coulombic potential.

\section{Treatments Based on Exponential SLEs}

Several authors have used the exponential form of the SLE to deal with the RYDMR lineshape problem. Applications have been made to triplet-triplet annihilation in molecular crystals, ${ }^{542}$ to photoconductivity in semiconductors, ${ }^{574}$ but mainly for charge recombination in photosynthetic reaction centers. ${ }^{93,742-745}$

Lesin et al..$^{542}$ investigated the RYDMR line shape of triplet-triplet annihilation in high magnetic field, when scanning the direction of the $B_{0}$ field through a highfield resonance. Actually, this corresponds to a variation of $\mathrm{S} / \mathrm{Q}_{0}$ coupling strength in (TT)-pair spin states, which goes through zero at the resonance direction. The exponential SLE set up in the laboratory frame was 
solved approximately by time-dependent expansion of the spin density matrix. An analytical solution was obtained for the limiting case of low singlet-pair reactivity with respect to the dissociation rate of the TT pair. The RYDMR line vanishes at the level-crossing position because here the kinetically active spin states are effectively pure singlet and quintet. In terms of Figure 51 the variation investigated would correspond to a decrease of the energy gap $\Delta\left(\mathrm{ST}_{0}\right)$, which in the present case corresponds to $\Delta\left(\mathrm{SQ}_{0}\right)$, whereas $\mathrm{T}_{+}$and $\mathrm{T}_{-}$ should be substituted by $Q_{1}$ and $Q_{-1}$.

The notion of spin conservation in geminate electron/hole pairs as a principal idea to explain resonant microwave effects on photoconductivity in semiconductors was introduced by Kaplan, Solomon, and Mott. ${ }^{573}$ The relation of this mechanism to the situation in radical pair theory has been recognized by Haberkorn and Dietz, ${ }^{574}$ who applied the exponential SLE method involving a $\Delta g$-type $\mathrm{S} / \mathrm{T}_{0}$ coupling.

In the rotating frame their SLE assumes the form

$$
\rho(t)=-i\left(H^{\prime} \rho-\rho H^{\dagger}\right)
$$

with the effective (non-Hermitian) Hamiltonian

$$
H^{\prime}=H_{\mathrm{M}}-1 / 2 i\left(k_{\mathrm{s}} Q_{\mathrm{s}}+k_{\mathrm{D}} I\right)
$$

the Hermitian part of which is given by

$H_{\mathrm{M}}=\left(\omega_{0}-\omega\right) S_{z}+\left(\omega_{0}^{\prime}-\omega\right) S_{z}{ }^{\prime}+\omega_{1} S_{x}+\omega_{1}{ }^{\prime} S_{x}{ }^{\prime}$

Here the primed quantities refer to the second spin of the $\mathrm{DD}$ pair. The parameters $k_{\mathrm{S}}$ and $k_{\mathrm{D}}$ denote the rate constants of singlet-pair recombination and pair dissociation, respectively.

In the case $\left(\omega_{0}-\omega_{0}^{\prime}\right) \gg k_{\mathrm{S}}, k_{\mathrm{D}}, \omega_{1}, \omega_{1}^{\prime}$ the solution of eq 234 corresponds to a well-resolved doublet of lines, for which an exact analytical line-shape formula has been given. The recombination yield ${ }^{F} Y_{S}$ as a function of $\omega$, e.g., in the range of the unprimed resonances, is ${ }^{\mathrm{F}} Y_{\mathrm{S}}=1 / 2 k_{\mathrm{S}}\left(k_{\mathrm{S}}+2 k_{\mathrm{D}}\right)^{-1}\left\{1+\kappa \omega_{1}^{2}\left[\left(\omega_{0}-\omega\right)^{2}+\left(k_{\mathrm{D}}+\right.\right.\right.$

$$
\left.\left.\left.k_{\mathrm{S}} / 4\right)^{2}\left(1+\omega_{1}^{2} / k_{\mathrm{D}}\left(k_{\mathrm{D}}+k_{\mathrm{S}} / 2\right)\right)\right]^{-1}\right\}(237)
$$

with

$$
\kappa=1 / 4\left(k_{\mathrm{S}} / k_{\mathrm{D}}\right)\left(k_{\mathrm{D}}+k_{\mathrm{S}} / 4\right)\left(k_{\mathrm{D}}+k_{\mathrm{S}} / 2\right)^{-1}
$$

Actually, this case treated by Haberkorn and Dietz with its limiting conditions for the analytical solution is kinetically equivalent to the situation treated with the integrated spin motion method by Kubarev and Pschenichnov. ${ }^{567}$ However, the relation between the results of these treatments is not obvious and has not been analyzed.

The need for an interpretation of RYDMR spectra observed with photosynthetic reaction centers has stimulated some theoretical work which has been based on the exponential SLE in the rotating frame (cf. eq 234).

Lersch and Michel-Beyerle ${ }^{93,742}$ derived a time-dependent result for the recombination yield of triplets starting from the formal solution to eq 234 :

$$
\rho(t)=\exp \left(-i H^{\prime} t\right) \rho(0) \exp \left(i H^{\prime} t\right)
$$

which has been evaluated by numerically diagonalizing the effective spin Hamiltonian matrix. RYDMR spectra were calculated for a two-proton model, taking into account the effect of exchange interaction $J$, anisotropic dipolar spin-spin interaction $D$, and variable microwave field $B_{1}$. Special emphasis was put on the analysis of $B_{1}$-dependence and anisotropy of the RYDMR spectra.

Tang and Norris ${ }^{743}$ presented a similar analysis of reaction center RYDMR spectra, derived from a numerical solution of the exponential SLE under stationary conditions. They used a Gaussian distribution of hyperfine couplings modeling the realistic situation in reaction centers. The results were averaged over a discrete orientational distribution of reaction centers. Special attention was paid to the relative influence of $B_{1}$ and singlet-triplet splitting parameters $J$ on the line shape. A maximum in the $B_{1}$ spectrum occurs if $B_{1}=$ $2 J$, the S-T energy gap.

Hunter et al..$^{74}$ and Lersch and Michel-Beyerle ${ }^{745}$ provided analytical expressions for the RYDMR line shape of reaction centers under the limiting condition of small hyperfine couplings, so that they may be treated as perturbations in the effective Hamiltonian. According to Lersch and Michel-Beyerle, the relative RYDMR intensity is given by

$$
\begin{gathered}
I_{\mathrm{RYDMR}} \equiv\left({ }^{\mathrm{s}} Y_{\mathrm{T}}\left(B_{0}, B_{1}\right)-{ }^{\mathrm{S}} Y_{\mathrm{T}}\left(B_{0}, 0\right)\right) /{ }^{\mathrm{s}} Y_{\mathrm{T}}\left(B_{0}, 0\right)= \\
\frac{1}{2} \frac{\omega_{1}^{2}}{\Omega^{2}}\left(\frac{J^{2}+\kappa^{2}}{(J+\Omega)^{2}+\kappa^{2}}+\frac{J^{2}+\kappa^{2}}{(J-\Omega)^{2}+\kappa^{2}}-2\right)
\end{gathered}
$$

Here

$$
\kappa=1 / 2\left(k_{\mathrm{S}}+k_{\mathrm{T}}\right)
$$

and $\Omega$ is given by eq $229 \mathrm{a}$ with $\Delta\left(\mathrm{ST}_{0}\right) \rightarrow 0$. Note, however, that the result (240) is not valid in the limit $k_{\mathrm{S}} / k_{\mathrm{T}} \rightarrow 0$. A corresponding correction is given in ref 745 .

Hunter et al. ${ }^{744}$ applied these solutions to fit experimental temperature-dependent RYDMR spectra. The observed RYDMR spectra were well reproduced by the assumption of temperature-dependent values of $J$ and the recombination rate constants $k_{\mathrm{S}}$ and $k_{\mathrm{T}}$. Lersch and Michel-Beyerle ${ }^{745}$ derived analytical conditions between the parameter values corresponding to characteristic limiting cases of the RYDMR line shape.

The RYDMR line-shape effects as determined by the ratio of microwave power and exchange interaction, discussed in ref 93 and $742-745$, may be qualitatively understood from the level crossing/anticrossing diagram depicted in Figure 52, illustrating the rationales given by Lersch and Michel-Beyerle ${ }^{745}$ and by Tang and Norris. ${ }^{743}$ The relative energies of the spin states are represented in the rotating frame as functions of $\omega$, whereby singlet and triplet mixing terms are considered as small perturbations, not to be seen in the diagram (actually the $\mathrm{S} / \mathrm{t}$ crossings would be weakly avoided). The $\mathrm{S} / \mathrm{T}$ splitting is indicated as $2 J$. The directions of spin quantization in the triplet eigenstates of $H_{\mathrm{M}}$ depend on $\omega$ and on the Rabi frequency $\omega_{1}$. Thus, whereas far from resonance $t_{+}, t_{0}$, and $t_{-}$correspond to $\mathrm{T}_{+}, \mathrm{T}_{0}$, and $\mathrm{T}_{-}$, quantized along $B_{0}$, at resonance $t_{+}, t_{0}$, and $\mathrm{t}_{-}$are completely quantized along $B_{1}$ so that here $T_{0}$ is equally distributed over $t_{+}$and $t_{-}$and $t_{0}$ is a $1: 1$ mixture of $T_{+}$and $T_{-}$. Since in a high $B_{0}$ field singlet-triplet transitions occur via $S / T_{0}$ coupling, the efficiency of this process as a function of $\omega$ will depend on how $T_{0}$ is distributed over the $t$ states and on the energy gaps between $S$ and $t_{+}, t_{0}$, and $t_{-}$in relation to $\omega_{1} .{ }^{745}$ The size of $\omega_{1}$ is expressed in the width of the $t_{+} / t_{-}$anticrossing at the resonance position. 

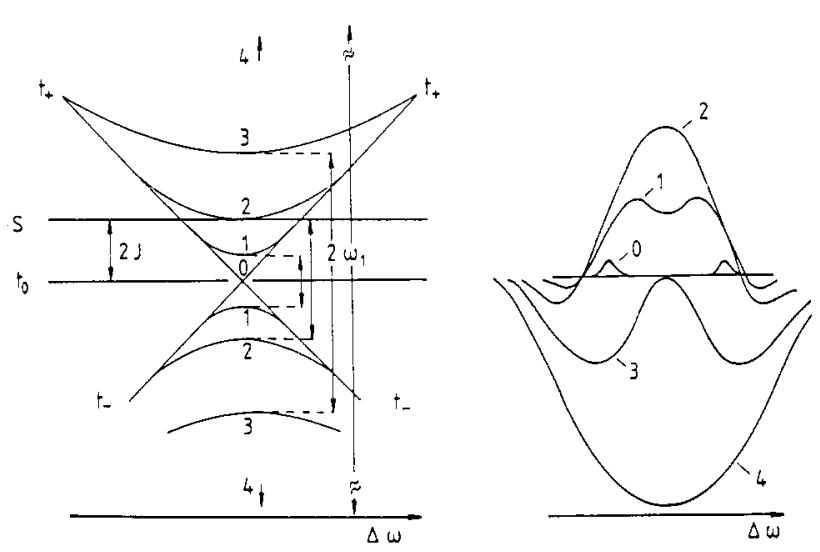

Figure 52. Schematic representation of RYDMR line-shape dependence of radical pairs on the ratio of microwave power (represented by various $\omega_{1}$ values) and exchange energy $J$. Left: energy scheme in rotating frame; right: typical line shapes. For details, cf. text.

Schematic RYDMR spectra corresponding to typical $\omega_{1} / J$ ratios are also depicted in Figure 52 . As $\omega_{1}$ tends toward zero (spectrum 0), two weak, well-separated RYDMR lines should appear at $\omega=\omega_{0} \pm 2 J$ due to the level crossing of $t_{+}$with the singlet level. As $\omega_{1}$ is increased (spectrum 1 ), the redistribution of $T_{0}$ from $t_{0}$ to $t_{1}$ under the effect of $\omega_{1}$ begins in the $\omega$ region outside the $S / t_{+}$crossings and leads to a decrease of triplet formation and negative RYDMR intensity in the wings of the line. However, in the central part of the line the intensity increases because here $t_{+} / t_{-}$anticrossing shifts $t_{+}$closer to $S$, thus favoring $S / t_{+}$transitions. A maximum of the RYDMR line is reached at $w_{1}=2 J$ since this is the situation where $t_{+}$, which carries $50 \%$ of $T_{0}$ character, is degenerate with $S$ at $\omega=\omega_{0}$. As $\omega_{1}$ increases further, the center of the line decreases (spectrum 3) and finally inverts completely, leading to a single, purely negative RYDMR line (spectrum 4).

\section{F. Paramagnetic and Magnetocatalytic Ortho/Para-Hydrogen Conversion}

The interconversion of hydrogen molecules with singlet nuclear spin alignment $\left(p-\mathrm{H}_{2}\right)$ and triplet nuclear spin alignment $\left(o-\mathrm{H}_{2}\right)$ may be achieved either by dissociative mechanisms or by paramagnetic catalysis. Only the latter mechanism will be considered here. It should be mentioned, though, that the principles of the radical pair mechanism may cause magnetic field effects also in the former case. ${ }^{560,692}$

According to Wigner, ${ }^{82}$ the paramagnetic conversion mechanism is due to the inhomogeneous magnetic field $\mathbf{B}(\mathbf{r})$ exhibited by the magnetic dipole of the paramagnetic collision partner, more precisely, to the antisymmetric part of the following nuclear spin Hamiltonian:

$$
\begin{aligned}
H= & g_{N} \mu_{N}\left(\mathbf{B}\left(\mathbf{r}_{1}\right) \boldsymbol{I}_{1}+\mathbf{B}\left(\mathbf{r}_{2}\right) \boldsymbol{I}_{2}\right)=g_{N} \mu_{N}\left[\frac { 1 } { 2 } \left(\mathbf{B}\left(\mathbf{r}_{1}\right)+\right.\right. \\
& \left.\left.\mathbf{B}\left(\mathbf{r}_{2}\right)\right)\left(\boldsymbol{I}_{1}+\boldsymbol{I}_{2}\right)+1 / 2\left(\mathbf{B}\left(\mathbf{r}_{1}\right)-\mathbf{B}\left(\mathbf{r}_{2}\right)\right)\left(\boldsymbol{I}_{1}-\boldsymbol{I}_{2}\right)\right]
\end{aligned}
$$

The second term is antisymmetric with respect to an interchange of both positions and spins of the $\mathrm{H}_{2}$ nuclei. Thus it couples ortho with para spin states and also odd with even rotational states.

Recently, another coupling mechanism of ortho and para hydrogen stats in a collision of $\mathrm{H}_{2}$ with a paramagnetic particle has been suggested by Ilisca and $\mathrm{Su}-$ gano. ${ }^{81}$ They considered a second-order coupling process involving (i) paramagnetic exchange between $\mathrm{H}_{2}$ and the paramagnetic catalyst, coupling the ${ }^{1} \Sigma_{\mathbf{g}}{ }^{+}(L=$ $0, I=0)$ electronic ground state to a ${ }^{3} \Sigma_{\mathrm{u}}{ }^{+}(L=1, I=$ 0 ) excited state of $\mathrm{H}_{2}$ (the so-called $\mathrm{X}$ process) whereby the rotational quantum number is changed by 1 , and (ii) nonsecular hyperfine coupling in $\mathrm{H}_{2}$, whereby ${ }^{3} \Sigma_{\mathrm{u}}{ }^{+}$ $(L=1, I=0)$ is coupled to ${ }^{3} \Sigma_{\mathrm{g}}{ }^{+}(L=1, I=1)$ with a change in the nuclear spin state (the so-called Y process). It is argued that the new XY (or YX) mechanism is more efficient than the Wigner (W) mechanism, if the exchange interaction exceeds a moderate value.

Since $o / p-\mathrm{H}_{2}$ conversion, irrespective of the type of coupling, requires exchange of an amount of energy, corresponding to one rotational quantum of about 100 $\mathrm{cm}^{-1}$, with the translational degrees of freedom of the collision partners, the transition cannot be realistically described as an isolated process in spin space only, as in the case with the radical pair mechanism, where the application of a spin-motion picture is most convenient. Nevertheless, there have been some attempts in the literature to visualize the $o / p-\mathrm{H}_{2}$ conversion process in the spin-motion picture.

Petzinger and Scalapino ${ }^{746}$ considered the nuclear spin motion of $\mathrm{H}_{2}$ in a stationary inhomogeneous magnetic field. As in the case of radical pairs, it is possible to distinguish the contributions of the rephasing mechanism (magnetic field at both spins has equal direction but different strength) and the spin-flip mechanism (different direction of local magnetic fields). The same model has been applied and extended by Jugel et al. ${ }^{155}$ to give a basic account of the so-called magnetocatalytic $o / p-\mathrm{H}_{2}$ conversion, whereby the catalytic influence of paramagnetic surfaces is modified by an external magnetic field. The model, which does not take into account the energetic requirements of $o / p-\mathrm{H}_{2}$ conversion and the reverse effect of the proton spins on the perturbing paramagnetic spins, is too crude to give reasonable quantitative estimates of the experimental effects observed.

The first quantitative account of the rate of paramagnetic $o / p-\mathrm{H}_{2}$ conversion was given by Wigner. ${ }^{82} \mathrm{He}$ considered collisions of $\mathrm{H}_{2}$ molecules with paramagnetic molecules in the gas phase, approximating the collisions by short periods of contact wherein the paramagnetic perturbation experienced by the $\mathrm{H}_{2}$ nuclei is considered as constant. The following expression was derived for the probability of a $\{$ para, $L=0\}$ to $\{$ ortho, $L=1$ \} transition in a collision:

$$
W(p(0) \rightarrow o(1))=\frac{b^{2} \mu_{\mathrm{a}}{ }^{2} \mu_{\mathrm{p}}{ }^{2}}{6 \hbar^{2} a^{6} v^{2}}
$$

Here $a$ is the collision distance, $b$ is the $\mathrm{H}_{2}$ internuclear distance, $v$ is the relative velocity of the collision, and $\mu_{\mathrm{a}}$ and $\mu_{\mathrm{p}}$ are the magnetic moments of the paramagnetic molecule and proton, respectively. With $a=1-2$ $\AA$ this expression yields single-collision probabilities on the order of $10^{-11}-10^{-13}$, which corresponds to the order of magnitude of the experimental results.

Kalckar and Teller ${ }^{747}$ afforded the following expression for the ratio of paramagnetic conversion rates of $o / p-\mathrm{H}_{2}$ and $o / p-\mathrm{D}_{2}$ (note that in $\mathrm{D}_{2}$ ortho states correspond to nuclear singlets and quintets and are combined with even rotational states, whereas para states 
correspond to nuclear triplets and are combined with odd rotational states ${ }^{748}$ ):

$$
\frac{k_{\mathrm{H}}(T)}{k_{\mathrm{D}}(T / 2)}=2\left(\frac{\mu_{\mathrm{p}} I_{\mathrm{d}}\left(2 I_{\mathrm{p}}+1\right)}{2 \mu_{\mathrm{d}} I_{\mathrm{p}}\left(2 I_{\mathrm{d}}+1\right)}\right)^{2}
$$

In eq $243 I_{\mathrm{d}}=1$ and $I_{+}=1 / 2$ are the spins and $\mu_{\mathrm{d}}$ and $\mu_{\mathrm{p}}$ the magnetic moments of deuteron and proton, respectively. In the derivation of eq 243 details of the collision dynamics have been omitted since these were considered to contribute equal factors to the rates, if these are compared at temperatures $T$ and $T / 2$, where thermal distributions of momenta in $\mathrm{H}_{2}$ and $\mathrm{D}_{2}$ are equal.

Farkas and Garbatski ${ }^{749}$ used both a generalized Wigner formalism for thermally averaged conversion rates of $o / p-\mathrm{H}_{2}$ and $o / p-\mathrm{D}_{2}$ and the Kalckar and Teller expression, eq 243, to evaluate the ratio of the magnetic moments of proton and deuteron from their experimental rates. The result from the Wigner treatment corresponded more closely to the accepted ratio of the magnetic moments than that obtained from the Kalckar and Teller expression.

The most advanced treatment of paramagnetically catalyzed $o / p-\mathrm{H}_{2}$ conversion in the gas phase has been given by Nielson and Dahler, ${ }^{750}$ who applied a full scattering theory formalism. Numerical results were obtained for $\mathrm{O}_{2}$ as catalyst using a hard-core intermolecular potential. The absolute rates obtained theoretically are by a factor of 3-4 smaller than the experimental ones. Possibly these results may be further improved by applying the new XY-coupling mechanism $^{81}$ instead of the Wigner mechanism.

The catalytic $o / p-\mathrm{H}_{2}$ conversion on paramagnetic surfaces was initially treated in a way similar to Wigner's gas-phase theory, however taking into account that the adsorbed hydrogen molecules undergo more contacts with paramagnetic centers than in the gas phase. ${ }^{751,752}$ Including the adsorption equilibrium may cause an overall negative energy of activation for reaction rates catalyzed by paramagnetic surfaces ${ }^{751}$ whereas the Wigner mechanism in the gas phase should yield rates monotonically increasing with temperature.

In a theoretical treatment by Leffler ${ }^{753}$ the $o / p-\mathrm{H}_{2}$ conversion mechanism on magnetic surfaces is ascribed to different nuclear relaxation rates of the two $\mathrm{H}_{2}$ protons, caused by their different distance from the paramagnetic surface centers (it has been assumed that the $\mathrm{H}_{2}$ molecules are oriented perpendicular to the surface). The paramagnetic spins are thought to induce proton nuclear relaxation by dipolar spin coupling which is rapidly modulated with a correlation time characteristic of the paramagnetic spin relaxation. The absolute values estimated by this method are larger than the experimental ones. The conclusion that a paramagnetic catalyst should be more efficient the longer the paramagnetic spin relaxation rate has been criticized by Ilisca et al., ${ }^{754,755}$ who derived a rate expression for catalytical $o / p-\mathrm{H}_{2}$ conversion on a magnetic surface by time-integrating the correlation function of perturbation matrix elements providing the coupling between appropriate rotational levels of $o-\mathrm{H}_{2}$ and $p-\mathrm{H}_{2}$. The transition rate was

$$
W_{o / p} \propto 2 \tau /\left(1+\omega_{o p}{ }^{2} \tau^{2}\right)
$$

where $\omega_{o p}$ is the frequency corresponding to the $o / p$ rotational energy difference and $\tau$ is a composite correlation time given by

$$
1 / \tau=1 / \tau_{\mathrm{C}}+1 / \tau_{\mathrm{P}}
$$

with $\tau_{\mathrm{C}}$, the diffusional correlation time of the hydrogen molecules with respect to the paramagnetic centers on the surface and $\tau_{\mathrm{p}}$, the paramagnetic relaxation time of the spin component vertical to the surface. The shorter time of both determines the motional degree of freedom providing most of the energy to be exchanged in the $o / p-\mathrm{H}_{2}$ conversion process.

The time-correlation functional approach has been applied also by Petzinger and Scalapino, ${ }^{746}$ who made use of a tensor operator formalism to handle dipolar interactions and correlation functions. They obtained absolute rates in terms of physical parameters that are helpful to analyze experimental results. Thus the motion of the $\mathrm{H}_{2}$ molecule on the surface was treated as a two-dimensional Maxwell-Boltzmann gas or by two-dimensional diffusion. Otherwise the rate of desorption was assumed to determine the translational correlation function of $\mathrm{H}_{2}$. A similar theoretical approach was used by Atkins and Clugston ${ }^{756}$ to deal with catalytic $o / p-\mathrm{H}_{2}$ conversion in solution. The results have been applied to determine the first-hydration shell radii of transition-metal ions from their catalytic activities in $o / p-\mathrm{H}_{2}$ conversion.

Special efforts toward an understanding of the external magnetic field modulation of the catalytic $o / p-\mathrm{H}_{2}$ by solid magnetic surfaces have been made by Ilisca and co-workers. ${ }^{83,754,757-759}$ In ferromagnetics such a change in the catalytic activity in a magnetic field may be due to a change of the magnetic polarization director with respect to the magnetic surfaces whereby a significant change in the amplitude of the time correlation function of the paramagnetic spin component vertical to the surface may ensue which is responsible for the $o / p-\mathrm{H}_{2}$ conversion process. ${ }^{757}$

More recently, Ilisca et al. ${ }^{83,758}$ gave a very detailed tensorial analysis of the theoretical rate of $o / p-\mathrm{H}_{2}$ conversion on paramagnetic surfaces. This approach was based on the Wangness and Bloch expression ${ }^{760}$ for the rate of irreversible processes. The quantum degrees of freedom that are explicitly taken into account are the $\mathrm{H}_{2}$ rotational angular momentum $(L)$, nuclear spin $(I)$, and the electronic spin of the paramagnetic center $(S)$. Allowance for a polarization of the partial density matrix in the space of these dynamic variables is made, whereas the density matrix of the $\mathrm{H}_{2}$ translational motion is assumed to correspond to the thermal equilibrium throughout. The conversion rate may be cast in the form

$$
W_{o p}=\operatorname{Tr}\{E \rho\}
$$

where $\rho$ is the stationary state density matrix in the basis of $L, I$, and $S$ eigenstates and $E$ is a reaction efficiency operator. An expansion of this expression into a sum of irreducible tensor operators products is performed, which forms a natural basis for a physically meaningful decomposition of the rate into contributions of various multipole polarizations and reactivities. A major conclusion from the analysis is that with $\rho$ corresponding to thermal equilibrium the order of magnitude of the experimentally observed magnetic field effects cannot be explained. Thus one has to conclude 
that in the steady state of the reaction there is a considerable degree of alignment of paramagnetic spins and nuclear spins (SI polarization) or of rotational angular momentum and nuclear spins ( $L I$ polarization). It is also pointed out that, whereas the antisymmetric part of the interaction Hamiltonian (cf. eq 241) is important for effecting ortho/para transitions, the symmetric part is likewise of importance in that it is responsible for the dynamic polarization effects mentioned. This important feature had not been noticed before.

A specific model explaining how such polarizations may arise has been described by Ilisca and Debauche. ${ }^{759}$ They suggested that the impinging $\mathrm{H}_{2}$ molecules librate on the catalyst surface, thus creating a fluctuating magnetic field that polarizes the neighboring magnetic impurities. These polarizations are maintained by the continuous exchange of the $\mathrm{H}_{2}$ molecules. Application of an external magnetic field leads to an enhancement of the polarization and hence also of the catalytic rate.

\section{Conclusion}

In this review we have tried to give an adequate and clearly structured, comprehensive representation of the present scope of the field of magnetokinetics, whereby we have attempted to put equal weights on both the theoretical and the experimental point of view. Attention was focused on the basic mechanistic principles and their mutual relations, on the diversity of experimental methods applied to trace magnetokinetic effects, on the great variety of chemical systems, where they appear, and finally on the theoretical techniques developed for their qualitative and quantitative understanding. We hope that the frontiers of these developments have been made clear and that the review will stimulate further research leading toward an increasing coherence of the field and a growing utilization of its innovation potential. Looking into the near future, we believe there is reason to anticipate substantial progress, e.g., along the following lines.

Experimental methods will certainly continue to be improved with respect to sensitivity and reproducibility in various domains of time resolution. If it became possible to achieve routine access to magnetic field effects below $1 \%$, this would greatly extend the general applicability of magnetokinetics as a tool for investigating reaction mechanisms.

There are already some examples of magnetic-fielddependent enzymatic reactions (cf. section IV.F). An increase of experimental accuracy and a systematic search for magnetokinetic effects with enzyme-catalyzed reactions might provide more evidence of radical pair type intermediates in such reactions and contribute also to a better understanding of magnetobiological effects.

The fascination of magnetokinetic effects, especially as far as the radical pair mechanism is concerned, results mainly from the fact that quite feable magnetic interactions with the electron spins are capable of controlling chemical reactions or related molecular processes wherein electronic energies, many orders of magnitude larger than these magnetic interactions, are transformed into vibrational energy. The systematic search for optimum -conditions to observe and utilize such effects has been, and should continue to be so, a great challenge for synthetic chemists to synthesize "supramolecular" arrangements as the chemically linked electron donor-acceptor systems. Radical pairs in suitable microreactors can be also conceived as examples of such supramolecular chemistry.

Whereas theories of the radical pair mechanism in homogeneous solutions appear to be well developed, there is so far no quantitative understanding of the magnetic field dependence of geminate radical pair kinetics in micellar and microemulsion type supercages. Pertinent theoretical developments are needed, which would in particular have to focus on the magnetic field dependence (up to high fields) of various mechanisms of electron spin relaxation. A novel access to the details of rotational and translational diffusion of radical pairs should result from an application of these theories to magnetokinetic effects in such media.

As for the magnetic isotope effect ensuing from the radical pair mechanism, there remains a wide field of isotopes to be explored throughout the periodic table. Finding convenient chemical systems and reactions to exploit such effects for magnetic isotope separation should represent a great challenge to chemists and photochemists.

There is recent evidence that the magnetic coupling of spin and orbital momentum in orbitally degenerate states of transition-metal complexes ${ }^{116,297,298,301,481,772-774}$ may cause appreciable magnetokinetic effects at fields in the range of several tesla. The intriguing theoretical problem about this type of phenomena is the way spectroscopic information, mainly available from lowtemperature paramagnetic resonance and magnetooptical investigations in the solid state, can be utilized in an appropriate dynamical description including the role of molecular motion during chemical reactions in liquid solutions.

These examples may suffice to substantiate our conviction that magnetokinetics will continue to establish itself as a distinguished field of chemical research, being attractive in its own right and a useful tool for investigating and furthering our understanding of the details of chemical dynamics.

Acknowledgments. We are indebted to Dr. K. A. McLauchlan for critically reading the manuscript and for helpful comments. Financial support by the Deutsche Forschungsgemeinschaft and by the Fonds der Chemischen Industrie is gratefully acknowledged.

\section{References}

(1) Bhatagnar, S. S.; Mathur, R. N.; Kapur, R. N. Philos. Mag. $1929,8,457$.

(2) Selwood, P.W. Chem. Rev. 1946, 38, 41.

(3) Figueras Roca, F. Ann. Chim. 1967, 2, 255.

(4) Avakian, P.; Merrifield, R. E. Mol. Cryst. 1968, 5, 37.

(5) Kaptein, R. "Chemically Induced Dynamic Nuclear Polarization", Doctoral Thesis, Leiden, The Netherlands, 1971.

(6) Buchachenko, A. L.; Zhidomirov, F. M. Russ. Chem. Rev. (Engl. Transl.) 1971, 40, 801 .

(7) Merrifield, R. E. Pure Appl. Chem. 1971, 27, 481.

(8) Ward, H. R. Acc. Chem. Res. 1972, 5, 18.

(9) Lawler, R. G. Acc. Chem. Res. 1972, 5,25

(10) Lepley, A. R.; Closs, G. L. Chemically Induced Magnetic Polarization; London: Wiley, 1978.

(11) Lawler, R. G. Prog. Nucl. Magn. Reson. Spectrosc. 1973, 9 , 145.

(12) Sokolik, I. A.; Frankevich, E. L. Usp. Fiz. Nauk 1973, 111, 261.

(13) Swenberg, Ch. E.; Geacintov, N. E. In Organic Molecular Photophysics; Birks, J. B., Ed.; New York, Wiley: 1973; Vol. $1, \mathrm{p} 489$. 
(14) Glarum, S. H. In ref $10, \mathrm{p} 1$

(15) Atkins, P. W.; McLauchlan, K. A. In ref 10, p 41.

(16) Avakian, P. Pure Appl. Chem. 1974, 37, 1.

(17) Atkins, P. W.; Lambert, T. P. Annu. Rep. Prog. Chem. 1975 , $72 A, 67$.

(18) Freed, J. H.; Pedersen, J. B. Adv. Magn. Reson. 1976, 8, 1.

(19) Buchachenko, A. L. Russ. Chem. Rev. (Engl. Transl.) 1976, 45,761 .

(20) Atkins, P. W. Evans, G. T. Adv. Chem. Phys, 1976, 35, 1.

(21) Faulkner, L. R. Int. Rev. Sci., Phys. Chem. Ser. 2 1976, 9 , 213.

(22) Sagdeev, R. Z.; Salikhov, K. M.; Molin, Y. M. Russ. Chem. Rev. (Engl. Transl.) 1977, 46, 569.

(23) Muus, L. T.; Atkins, P. W.; McLauchlan, K. A.; Pedersen, J. B. Eds. Chemically Induced Magnetic Polarization; Reidel: Dordrecht, The Netherlands, 1977.

(24) Küttner, H. G.; Selzle, H. L.; Schlag, E. W. Isr. J. Chem. $1977,16,264$.

(25) Geacintov, N. E.; Swenberg, C. E. In Luminescence Spectroscopy; Lumb, M. D. Ed.; Academic: London, 1978; p 239.

(26) Selwood, P. W. Adv. Catal. 1978, 27, 23.

(27) Hore, P. J.; McLauchlan, K. A. Rev. Chem. Intermed. 1979, 3,89 .

(28) Pedersen, J. B. Theories of Chemically Induced Magnetic Polarization; Odense University Press, 1979.

(29) Lin, S. H.; Fujimura, Y. In Excited States; Lim, E. C., Ed.; Academic: New York, 1979; Vol. 4, p 237.

(30) Adrian, F. J. Rev. Chem. Intermed. 1979, 3, 3.

(31) Molin, Yu. N.; Sagdeev, R. Z.; Salikhov, K. M. Sov. Sci. Rev., Sect. B, Chem. Rev. 1979, 1, 67 .

(32) Schott, M. In Light-Induced Charge Separation in Biology and Chemistry; Gerischer, H., Katz, J. J., Eds.; Dahlem Konferenzen: Berlin, FRG, 1979; p 109.

(33) Brocklehurst, B. Daresbury Lab. Rep. 1979, DL/SCI/R 13, 48.

(34) Hoff, A. J. Phys. Rep. 1979, 54, 75.

(35) Wan, J. K. S. Adv. Photochem. 1980, 12, 283

(36) Turro, N. J.; Kräutler, B. Acc. Chem. Res. 1980, 13, 369.

(37) Hoff, A. J. Q. Rev. Biophys. 1981, 14, 599.

(38) McLauchlan, K. A. Sci. Prog. 1981, 67, 509.

(39) Turro, N. J. Pure Appl. Chem. 1981, 53, 259

(40) Street, R. A. Adv. Phys. 1981, 30, 593.

(41) Frankevich, E. L.; Kubarev, S. I. In Triplet State ODMR Spectroscopy; Clarke, R. H., Ed.; Wiley: New York, 1982; p 138.

(42) Turro, N. J.; Kräutler, B. In Diradicals; Borden, W. T., Ed.; Wiley: New York, 1982; p 259.

(43) Schulten, K. In Festkörperprobleme (Adv. Solid State Phys., Vol. XXII) Treusch, J., Ed.; Vieweg: Braunschweig, FRG), 1982; p 83.

(44) Hoff, A. J. Biophys. Struct. Mech. 1982, 8, 107.

(45) Boxer, S. G.; Chidsey, C. E. D.; Roelofs, M. G. Annu. Rev. Phys. Chem. 1983, 34, 389

(46) Brocklehurst, B. Radiat. Phys. Chem. 1983, 21, 57.

(47) Turro, N. J.; Weed, G. C. J. Am. Chem. Soc. 1983, 105, 1861.

(48) Turro, N. J. Proc. Natl. Acad. Sci. U.S.A. 1983, 80, 609.

(49) Nagakura, S.; Hayashi, H. H. Radiat. Phys. Chem. 1983, 21, 91.

(50) Buchachenko, A. L. Prog. React. Kinet. 1984, 13, 163.

(51) Hoff, A. J. Q. Rev. Biophys. 1984, 17, 153.

(52) Salikhov, K. M.; Molin, Yu. N.; Sagdeev, R. Z.; Buchachenko, A. L. Spin Polarization and Magnetic Effects in Radical Reactions; Elsevier: Amsterdam, The Netherlands, 1984.

(53) Gould, I. R.; Turro, N. J.; Zimmt, M. B. Adv. Phys. Org. Chem. 1984, 20, 1.

(54) Turro, N. J.; Kräutler, B. Isotopes Org. Chem. 1984, 6, 107.

(55) Buckley, C. D.; McLauchlan, K. A. Mol. Phys. 1985, 54, 1.

(56) Brocklehurst, B. Int. Rev. Phys. Chem. 1985, 4, 279.

(57) Hoff, A. J. Photochem. Photobiol. 1986, 43, 727.

(58) Maret, G.; Kiepenheuer, J.; Boccara, N., Eds. Biophysical Effects of Steady Fields (Springer Proc. Phys., Vol. 11); Springer: Berlin, 1986

(59) Glasstone, S.; Laidler, K. J.; Eyring, $\mathrm{H}$. The Theory of Rate Processes; McGraw-Hill: New York, 1941.

(60) Freed, K. F. In Radiationless Processes in Molecules in Condensed Phases; Fong, F. K., Ed.; Springer: Berlin, 1976; o 23 (Top. Appl. Phys. Vol. 15).

(61) Atkins, P. W.; Stannard, P. R. Chem. Phys. Lett. 1977, 47, 113.

(62) Atkins, P. W. Chem. Phys. Lett. 1966, 66, 403

(63) Syage, J. A Chem. Phys. Lett. 1982, 91,378.

(64) Monchick, L.; Adrian, F. J. J. Chem. Phys. 1978, 68, 4376.

(65) Schulten, K.; Wolynes, P. J. Chem. Phys. 1978, 68, 3292.

(66) Chen, C. P.; Heinsohn, R. J.; Mulay, L. N. J. Phys. Soc. Jpn. $1968,25,319$

(67) Sakaguchi, Y.; Hayashi, H.; Nagakura, S. Bull. Chem. Soc. Jpn. 1980, 53, 39 .

(68) Schulten, K.; Epstein, I. R. J. Chem. Phys. 1979, 71, 309.

(69) Lendi, K.; Gerber, P.; Labhart, H. Chem. Phys, 1977, 20, 145.

(70) Lesin, V. I. Phys. Status Solidi B: 1978, 90, K13.
(71) Merrifield, R. E. J. Chem. Phys. 1968, 48, 4318.

(72) Michel, C.; Tric, C. Chem. Phys. 1980, 50, 341.

(73) Johnson, R. C.; Merrifield, R. E. Phys. Rev. B 1970, 1, 896

(74) Shain, A. L.; Sharnoff, M. Chem. Phys. Lett. 1972, 16, 503.

(75) Grant, A. I.; McLauchlan, K. A. Chem. Phys. Lett. 1983, 101, 120.

(76) Schwoerer, M.; Wolff, H. C. Proc. Colloq. Ampere 1966, 14, 87.

(77) De Groot, M. S.; Hesselmann, I. A. M.; Van der Waals, J. H. Mol. Phys. 1967, 12, 259 .

(78) Hall, L.; Armstrong, A.; El-Sayed, M. A. J. Chem. Phys. 1967, $48,1395$.

(79) Wong, K. S.; Hutchinson, D. A.; Wan, J. K. S. J. Chem. Phys. $1973,58,985$.

(80) Steiner, U. E. Ber. Bunsenges. Phys. Chem. 1981, 85, 228.

(81) Ilisca, E.; Sugano, S. Phys. Rev. Lett. 1986, 57, 2590.

(82) Wigner, E. Z. Phys. Chem. B 1933, 23, 28.

(83) Ilisca, E. Phys. Rev. Lett. 1978, 40, 1535.

(84) Kaptein, R.; Oosterhoff, J. L. Chem. Phys. Lett. 1969, 4, 195.

(85) Atkins, P. W.; Gurd, C.; McLauchlan, K. A.; Simpson, A. F. Chem. Phys. Lett. 1971, 8, 55.

(86) Bargon, J.; Fischer, H.; Johnsen, U. Z. Naturforsch. 1967, $22 a, 1551$.

(87) Ward, H. R.; Lawler, R. G. J. Am. Chem. Soc, 1967, 89, 5518.

(88) Den Blenken, H. J.; van der Zwet, G. P.; Hoff, A. Chem. Phys. Lett. 1982, 85, 335 .

(89) Trifunac, A. D.; Evanochko, W. T. J. Am. Chem. Soc. 1980, 102,4598

(90) Von Schütz, J. U.; Steudle, W.; Wolff, H. C.; Yakhot, V. Chem. Phys. Lett. 1980, 46, 53 .

(91) Anisimov, O. A.; Grigoryants, V. M.; Molchanov, V. K.; Molin, Yu. N. Chem. Phys. Lett. 1979, 66, 265.

(92) Trifunac, A. D.; Smith, J. P. Chem. Phys. Lett. 1980, 73, 94.

(93) Lersch, W.; Michel-Beyerle, M. E. Chem. Phys. 1983, 78, 115.

(94) Lawler, R. G.; Evans, G. T. Ind. Chim. Belge 1971, 36, 1087

(95) Buchachenko, A. L.; Galimov, E. M.; Ershov, V. V. Dokl. Akad. Nauk. SSSR 1976, 228, 379.

(96) Sagdeev, R. Z.; Leshina, T. V.; Kamkha, M. A.; Belchenko, O. I.; Molin, Yu. N.; Rezvukhin, A. J. Chem. Phys. Lett. 1977, $48,89$.

(97) Hoff, A. J.; Lous, E. J.; Moehl, K. W.; Dijkman, J. A. Chem. Phys. Lett. 1985, 114, 39.

(98) Staerk, H.; Kühnle, W.; Treichel, R.; Weller, A. Chem. Phys. Lett. 1985, 118, 19

(99) Saik, V. A.; Lukzen, N. N.; Grigoryants, O. A.; Anisimov, A. B.; Doktorov, A. B.; Molin, Yu. N. Chem. Phys. 1984, 84, 421.

(100) Geschwind, S.; Devlin, G. E.; Cohen, R. L.; Chinn, S. R. Phys. Rev. 1967, 137, A1087.

(101) Sharnoff, M. J. Chem. Phys. 1967, 46, 3263.

(102) Kwiram, A. Chem. Phys. Lett. 1967, 1, 272.

(103) Schmidt, J.; Hesselmann, I. A. M.; de Groot, M. S.; van der Waals, J. H. Chem. Phys. Lett. 1967, 1, 434.

(104) Tinti, D. S.; El-Sayed, M. A.; Maki, A. H.; Harris, C. B. Chem. Phys, Lett. $1969,3,343$

(105) Brossel, J.; Bitter, F. Phys. Rev. 1952, 86, 308

(106) Frankevich, E. L.; Pristupa, A. I.; Lesin, V. I. Chem. Phys. Lett. 1977, 47, 304

(107) Moehl, K. W.; Lous, E. J.; Hoff, A. J. Chem. Phys. Lett. 1985, $121,22$.

(108) Biegeleisen, D. K.; Knights, J. C.; Street, R. A.; Tsang, C.; White, R. M. Philos. Mag. 1978, B37, 477.

(109) Ziegler, J.; Karl, N. Chem. Phys. 1979, 40, 207.

(110) Sagdeev, R. Z.; Grishin, Yu. A.; Gogolev, A. Z.; Dooshkin, A. V.; Semenov, A. G.; Molin, Yu. N. Zh. Strukt. Khim. 1979, 20,1132 .

(111) Berdinskii, V. L.; Muchachenko, A. L. Russ. J. Phys. Chem. (Engl. Transi.) 1981, 55, 1032.

(112) Bube, W.; Haberkorn, R.; Michel-Beyerle, M. E. J. Am. Chem. Soc. 1978, 100, 5993.

(113) Tribel, M. M.; Morozov, A. K.; Leksin, A. N.; Frankevich, E. L. Dokl. Akad. Nauk SSSR 1985, 284, 1170.

(114) Herve, P.; Nome, F.; Fendler, J. H. J. Am. Chem. Soc. 1984, $106,8291$.

(115) Kohlrausch, F. Praktische Physik; Kohlrausch, F., Lautz, G., Taubert, T., Eds.; Teubner: Stuttgart, FRG, 1968; Vol. 2, p 255 .

(116) Frink, M. E.; Geiger, D. K.; Ferraudi, G. J. J. Phys. Chem. 1986, 90,1924

(117) Cavenett, B. C. In Luminescence Spectroscopy; Lumb, M. D., Ed.; Academic: London: 1978; p 299.

(118) Montgomery, D. B. Solenoid Magnetic Design; Wiley: New York, 1969.

(119) Turro, N. J.; Chung, Ch.-J.; Jones, G., II; Becker, W. G. J. Phys. Chem. 1982, 86, 3677 .

(120) Lombardi, M.; Jost, R.; Michel, C.; Tramer, A. Chem. Phys. $1980,46,273$.

(121) Grant, A. I.; McLauchlan, K. A.; Nattrass, S. R. Mol. Phys. $1985,55,557$.

(122) Bowman, M. K.; Budil, D. E.; Closs, G. L.; Kostka, A. G.; Wraight, C. A.; Norris, J. R. Proc. Natl. Acad. Sci. U.S.A. 
$1981,78,3305$.

(123) Wasielewski, M. R.; Norris, J. R.; Bowman, M. K. Faraday Discuss. Chem. Soc. 1984, 78, 279.

(124) Trifunac, A. D.; Norris, J. R.; Lawler, R. G. J. Chem. Phys. $1979,71,4380$.

(125) Molin, Yu. N.; Anisimov, O. A.; Grigoryants, V. M.; Molchanov, V. K.; Salikhov, K. M. J. Phys. Chem. 1980, 84, 1853.

(126) Hardy, W. N.; Whitehead, L. A. Rev. Sci. Instrum. 1981, 52, 213.

(127) Falconer, W. E.; Wasserman, E. J. Chem. Phys. 1966, 45, 1843.

(128) Sorokin, N. I.; Makarov, V. I.; Lavrik, N. L.; Gusev, Yu. M.; Skubnevskaya, G. I.; Bazhin, N. M.; Molin, Yu. N. Chem. Phys. Lett. 1981, 78, 8 .

(129) Stich, E. M.; Baumeister, W. F.; Huber, J. R. Chem. Phys. Lett. 1984, 108, 466 .

(130) Tanimoto, Y.; Hayashi, H.; Nagakura, S.; Sakuragi, H.; Tokomaru, K. Chem. Phys. Lett. 1976, 41, 267.

(131) Baretz, B. H.; Turro, N. J. J. Am. Chem. Soc. 1983, 105, 1309

(132) Kurskii, Yu. A.; Baryshnikov, Yu. N.; Vesnovskaya, G. I.; Koloshina, N. N.; Aleksandrov, Yu. A. Dokl. Akad. Nauk SSSR 1981, 258, 936 .

(133) Hata, N. Chem. Lett. 1976, 547.

(134) Hata, N. Chem. Lett. 1978, 1359

(135) Hata, N.; Ono, Y.; Nakagawa, F. Chem. Lett. 1979, 603

(136) Tanimoto, Y.; Takashima, M.; Itoh, M. J. Phys. Chem. 1984, 88,6053

(137) Sagdeev, R. Z.; Molin, Yu. N.; Salikhov, K. M.; Leshina, T. V.; Kamha, M. A.; Shein, S. M. Org. Magn. Reson. 1973, 5, 603.

(138) Podoplelov, A. V.; Sagdeev, R. Z.; Leshina, T. V.; Grishin, Yu. A.; Molin, Yu. N. Dokl. Akad. Nauk SSSR 1975, 225, 866.

(139) Khudyakov, I. V.; Prokof'ev, A. I.; Margulis, L. A.; Kuzmin V. A. Chem. Phys, Lett. 1984, 104, 409.

(140) Turro, N. J.; Chow, M.-F.; Rigaudy, J. J. Am. Chem. Soc. $1979,101,1302$.

(141) Turro, N. J.; Chow, M.-F. J. Am. Chem. Soc. 1979, 101, 3701.

(142) Turro, N. J.; Chow, M.-F.; Chung, C.-J.; Tung, C.-H. J. Am. Chem. Soc. $1980,102,7391$.

(143) Turro, N. J.; Chow, M.-F.; Chung, C.-J.; Kräutler, B. J. Am. Chem. Soc. 1981, 103, 3886

(144) Tarasov, V. F.; Buchachenko, A. L. Bull. Acad. Sci. USSR, Div. Chem. Sci. 1983, 68.

(145) Tarasov, V. F.; Buchachenko, A. L. Bull. Acad. Sci. USSR, Div. Chem. Sci. 1983, 72.

(146) Turro, N. J.; Chow, M.-F.; Kräutler, B. Chem. Phys, Lett. $1980,73,545$.

(147) Turro, N. J.; Chow, M.-F. J. Am. Chem. Soc. 1980, 102, 1190

(148) Turro, N. J.; Paczkowski, M. A.; Wan, P. J. Org. Chem. 1985 50,1399

(149) Tanimoto, Y.; Udagawa, H.; Katsuda, Y.; Itoh, M. J. Phys. Chem. 1983, 87, 3976.

(150) Tanimoto, Y.; Shimizu, K.; Itoh, M. J. Am. Chem. Soc. 1984, $106,7257$.

(151) Tanimoto, Y.; Shimizu, K.; Itoh, M. Photochem. Photobiol. $1984,39,511$.

(152) Schlenker, W.; Steiner, U. E. Ber. Bunsenges. Phys. Chem. $1985,89,1041$.

(153) Kiwi, J. J. Phys. Chem. 1983, 87, 2274.

(154) Selwood, P. W. J. Catal. 1971, 22, 123.

(155) Jugel, J.; Schwab, G.-M.; Voitländer, J. J. Chem. Phys. 1983, 78,4755 .

(156) Margulis, L. A.; Khudyakof, I. V.; Kuz'min, V. A. Chem. Phys. Lett. 1985, 119, 244.

(157) Michel-Beyerle, M. E.; Haberkorn, R.; Bube, W.; Steffens, E.; Schröder, H.; Neusser, H. J.; Schlag, E. W. Chem. Phys. 1976, $17,139$.

(158) Michel-Beyerle, M. E.; Scheer, H.; Seidlitz, H.; Tempus, D.; Haberkorn, R. FEBS Lett. 1979, 100, 9.

(159) Treichel, R.; Staerk, H.; Weller, A. Appl. Phys. 1983, B31, 15

(160) Ulrich, T.; Steiner, U. E.; Föll, R. E. J. Phys. Chem. 1983, 87, 1873.

(161) Hayashi, H.; Sakaguchi, Y.; Nagakura, S. Chem. Lett. 1980, 1149.

(162) Sakaguchi, Y.; Hayashi, H. Chem. Phys, Lett, 1982, 87, 539.

(163) Ulrich, T.; Steiner, U. E. Chem. Phys. Lett. 1984, 112, 365.

(164) Scaiano, J. C.; Lougnot, D.-J. Chem. Phys. Lett. 1984, 105 , 535.

(165) Brocklehurst, B. Faraday Discuss. Chem. Soc. 1977, 63, 96.

(166) Sargent, F. P.; Brocklehurst, B.; Dixon, R. S.; Gardy, E. M.; Lopata, V. J.; Singh, A. J. Phys. Chem. 1977, 81, 815.

(167) Anisimov, O. A.; Bizyaev, V. L.; Lukzen, N. N.; Grigoryants, V. M.; Molin, Yu. N. Chem. Phys. Lett. 1983, 101 131

(168) Veselov, A. V.; Melekhov, V. I.; Anisimov, O. A.; Molin, Yu. N. Chem. Phys. Lett. 1987, 136, 263.

(169) Klein, J. J. Chim. Phys. 1983, 80,627.

(170) Staerk, H.; Razi Naqvi, K. Chem. Phys. Lett. 1977, 50, 386.

(171) Tanimoto, Y.; Shimizu, K.; Itoh, M. Chem. Phys. Lett. 1984, $112,2217$.

(172) Tanimoto, Y.; Watanabe, T.; Nakagaki, R.; Hiramatsu, M.;
Nagakura, S. Chem. Phys. Lett. 1985, 116, 341.

(173) Frankevich, E. L. Faraday Discuss. Chem. Soc. 1971, 51, 37.

(174) Delannoy, P.; Schott, M. Phys. Lett. 1969, 30a, 357.

(175) Bube, W.; Michel-Beyerle, M. E.; Haberkorn, R.; Steffens, E. Chem. Phys. Lett. 1977, 50, 389.

(176) Müller, N.; Papier, G.; Charle, K. P.; Willig, F. Ber. Bunsenges. Phys. Chem. 1979, 83, 130.

(177) Solomon, I. Solid State Commun. 1976, 20, 215.

(178) Solomon, I.; Biegleisen, D.; Knights, J. C. Solid State Commun. 1977, 22, 505.

(179) Neubert, D.; Hoffmann, K.; Teichmann, H.; Schlief, R. Solid-State Electron. 1978, 21, 1445

(180) Steubing, W. Verh.d Dtsch. Phys. Ges. 1913, 15, 1181.

(181) Steubing, W. Ann. Phys. 1919, 58, 55; 1921, 64, 673.

(182) Wood, R. W.; Ribaud, G. Philos. Mag. 1914, 27, 1009.

(183) Oldenburg, O.Z. Phys. 1921, 6, 35.

(184) Franck, J.; Grotrian, W. Z. Phys. 1921, 6, 35.

(185) Turner, L. A. Z. Phys. 1930, 65, 464.

(186) Degenkolb, E. O.; Steinfeld, J. I.; Wassermann, E.; Klemperer, W. J. Chem. Phys. 1969, 51, 615.

(187) Chapman, G. D.; Bunker, P. R. J. Chem. Phys. 1972, 57, 2951 .

(188) Broyer, M.; Vigue, J.; Lehmann, J.-C. Chem. Phys. Lett. $1973,22,313$.

(189) Vigue, J.; Broyer, M.; Lehmann, J.-C. J. Phys. 1974, B7, L158.

(190) Vigue, J.; Broyer, M.; Lehmann, J.-C. J. Chem. Phys. 1975, 62,4941 .

(191) Niewodniczansky, H. Z. Phys. 1929, 55, 676

(192) Solarz, R.; Butler, S.; Levy, D. H. J. Chem. Phys. 1973, 58, 5172.

(193) Butler, S.; Kahler, C.; Levy, D. H. J. Chem. Phys. 1975, 62, 815

(194) Butler, S.; Levy, D. H. J. Chem. Phys. 1977, 66, 3538

(195) Makarov, V. I.; Lavrik, N. L.; Skubnevskaya, G. I.; Bazhin, N. M. React. Kinet. Lett. 1979, 12, 225.

(196) Makarov, V. I.; Lavrik, N. L.; Bazhin, N. M. Chem. Phys. $1982,72,213$.

(197) Matsuzaki, A.; Nagakura, S. Chem. Lett. 1974, 675.

(198) Matsuzaki, A.; Nagakura, S. Bull. Chem. Soc. Jpn. 1976, 49, 359

(199) Matsuzaki, A.; Nagakura, S. J. Lumin. 1976, 12/13, 787.

(200) Orita, H.; Morita, H.; Nagakura, S. Chem. Phys. Lett. 1981, $81,29$.

(201) Silvers, S. J.; McKeever, M. R.; Chawla, G. K. Chem. Phys. $1983,80,177$

(202) Sorokin, N. I.; Lavrik, N. L.; Skubnevskaya, G. I.; Bazhin, N. M.; Molin, Yu. N. Dokl. Akad. Nauk SSSR 1979, 245, 657 .

(203) Sorokin, N. I.; Lavrik, N. L.; Skubneskaya, G. I.; Bazhin, N M.; Molin, Yu. N. Nouv. J. Chim. 1980, 4, 395.

(204) Orita, H.; Morita, H.; Nagakura, S. Chem. Phys. Lett. 1981, 81,409

(205) Orita, H.; Morita, H.; Nagakura, S. Chem. Phys. Lett. 1982, $86,123$.

(206) Dong, R. Y.; Kroll, M., unpublished results cited by Dong, $R$. Y.; Ramsay, D. A. Can. J. Phys. 1973, 51, 1491

(207) Matsuzaki, A.; Nagakura, S. Chem. Phys. Lett. 1976, 37, 204.

(208) Matsuzaki, A.; Nagakura, S. Z. Phys. Chem. (Frankfurt/ Main) 1976, 101, 283

(209) Matsuzaki, A.; Nagakura, S. Helv. Chim. Acta 1978, 61, 675.

(210) Küttner, H. G.; Selzle, H. L.; Schlag, E. W. Chem. Phys. Lett. $1977,48,207$.

(211) Küttner, H. G.; Selzle, H. L.; Schlag, E. W. Chem. Phys. 1978, $28,1$.

(212) Nakamura, J.; Hashimoto, K.; Nagakura, S. J. Lumin. 1981, $24 / 25,763$

(213) Hashimoto, K.; Nagakura, S.; Nakamura, J.; Iwata, S. Chem. Phys. Lett. 1980, 74, 228.

(214) Henke, W.; Selzle, H. L.; Hays, T. R.; Schlag, E. W. Z. Naturforsch. 1980, 35a, 1271.

(215) Wakayama, N. I.; Ogasawara, I.; Hayashi, H. Chem. Phys. Lett. 1984, 105, 209.

(216) Hayashi, H. Bull. Chem. Soc. Jpn. 1984, 57, 1753.

(217) Hayashi, H. Chem. Phys. Lett. 1982, 87, 113.

(218) Wakayama, N. I.; Ogasawara, I.; Nishikawa, T.; Ohyagi, Y.; Hayashi, H. Chem. Phys. Lett. 1984, 107, 207.

(219) Fukuda, Y.; Hayashi, H.; Nagakura, S. Chem. Phys. Lett. $1985,119,480$

(220) Sorokin, N. I.: Bazhin, N. M.; Ermenchuk, G. G. Chem. Phys. Lett. 1983, 99,181 .

(221) Dushkin, A. V.; Yurkovskay, A. V.; Sagdeev, R. Z. Chem. Phys, Lett. 1979, 67, 524.

(222) Kato, H.; Onomichi, K. J. Chem. Phys. 1985, 82, 1642

(223) Van Vleck, J. H. Phys. Rev. 1932, 40, 544.

(224) Lyon, R. K. J. Am. Chem. Soc. 1964, 86, 1907.

(225) Imamura, T.; Tamai, N.; Fukuda, Y.; Yamazuaki, S.; Nagakura, S.; Abe, H.; Hayashi, H. Chem. Phys. Lett. 1987, 135, 208 .

(226) Ohta, N.; Fujita, M.; Baba, H. Chem. Phys. Lett. 1987, 135, 330 
(227) Sorokin, N. I.; Bazhin, N. M.; Dul'tsev, E. N. Chem. Phys. Lett. 1987, 138, 170.

(228) Sixl, H.; Schwoerer, M. Chem. Phys. Lett. 1970, 6, 21.

(229) Frankevich, E. L.; Balabanov, E. I. JETP Lett. 1965, 1, 169.

(230) Frankevich, E. L.; Balabanov, E. I.; Vselyubskaya, G. V. Sov. Phys.-Solid State (Engl. Transl.) 1966, 8, 1567.

(231) Frankevich, E. L.; Sokolik, I. A. Sov. Phys. JETP 1967, 25, 790.

(232) Morgan, K.; Pethig, R. Nature (London) 1967, 900.

(233) Geacintov, N. E.; Pope, M.; Fox, S. J. Phys. Chem. Solids $1970,31,1375$

(234) Frankevich, E. L.; Sokolik, I. A. Solid State Commun. 1970, $8,251$.

(235) Bouchriha, H.; Delacote, G.; Delannoy, P.; Schott, M. J. Phys. 1974, 35, 577 .

(236) Johnson, R. C.; Merrifield, R. E.; Avakian, P.; Flippen, R. B. Phys. Rev. Lett. 1967, 19, 285.

(237) Klein, G.; Voltz, R.; Schott, M. Chem. Phys. Lett. 1972, 16, 340.

(238) Klein, G.; Voltz, R.; Schott, M. Chem. Phys. Lett. 1973, 19 , 391.

(239) Albrecht, W. G.; Coufal, H.; Haberkorn, R.; Michel-Beyerle, M. E. Phys. Status Solid B: 1978, 89, 261.

(240) Rusin, B. A.; Rumyantsev, B. M.; Aleksandrov, I. V.; Frankevich, E. L. Phys. Status Solid 1969, 34, K103.

(241) Frankevich, E. L.; Sokolik, I. A.; Rumyantsev, B. M. High Energy Chem. 1972, 5, 318.

(242) Johnson, R. C.; Merrifield, R. E. Bull. Am. Phys. Soc. 1968 13,640 .

(243) Fourney, J.; Delacote, G. Chem. Phys. Lett. 1970, 5, 495.

(244) Smith, G. S.; Hughes, R. C. Phys. Rev. Lett. 1968, 20, 1358.

(245) Soos, Z. G. J. Chem. Phys. 1969, 51, 2107.

(246) Arnold, S. J. Chem. Phys. 1974, 61, 431.

(247) Frankevich, E. L.; Rumyantsev, B. M. JETP Lett. 1967, 6, 70.

(248) Schwob, H. P.; Williams, D. F. Chem. Phys. Lett. 1972, 13 , 581.

(249) Schwob, H. P.; Williams, D. F. J. Chem. Phys. 1973, 58, 1542.

(250) Geacintov, N. E.; Binder, M.; Swenberg, C. E.; Pope, M. Phys. Rev. 1975, B12, 4113

(251) Arnold, S.; Hu, W.; Pope, M. Mol. Cryst. Liq. Cryst. 1976, 36, 179.

(252) Ern, V.; McGhie, A. R. Mol. Cryst. Liq. Cryst. 1971, 15, 277.

(253) Klein, G.; Voltz, R. Int. J. Radiat. Phys. Chem. 1975, 7, 155.

(254) Fuchs, C.; Klein, J.; Voltz, R. Int. J. Radiat. Phys. Chem. $1983,21,67$.

(255) Klein, J.; Martin, P.; Voltz, R. Chem. Phys. Lett. 1983, 94, 10.

(256) Klein, J.; Martin, P.; Voltz, R. J. Lumin. 1981, 24/25, 99.

(257) Chabr, M.; Fünfschilling, J.; Zschokke-Gränacker, I. Chem. Phys. Lett. 1974, 25, 387

(258) Merrifield, R. E.; Avakian, P.; Groff, R. P. Chem. Phys. Lett. $1969,3,155$.

(259) Geacintov, N.; Pope, M.; Vogel, F. Phys. Rev. Lett. 1969, 22, 593.

(260) Pope, M.; Geacintov, N. E.; Vogel, F. Mol. Cryst. Liq. Cryst. $1969,6,83$.

(261) Groff, R. P.; Avakian, P.; Merrifield, R. E. J. Lumin. 1970 $1 / 2,218$.

(262) Groff, R. P.; Avakian, P.; Merrifield, R. E. Phys, Rev. B 1970, 1,815 .

(263) Moller, W. M.; Pope, M. J. Chem. Phys. 1973, 59, 2760.

(264) Kalinowski, J.; Godlewski, J. Chem. Phys. Lett. 1975, 36, 345.

(265) Geacintov, N. E.; Burgos, J.; Pope, M.; Strom, C. Chem. Phys. Lett. 1971, 11, 504

(266) Kalinowski, J.; Godlewski, J. Chem. Phys. Lett. 1974, 25, 499

(267) Arnold, S.; Swenberg, C. E.; Pope, M. J. Chem. Phys. 1976, 64,5115

(268) Arnold, S.; Alfano, R. R.; Hu, W.; Ho, P.; Selsby, R.; Tharrats, J.; Swenberg, C. E. J. Chem. Phys. 1976, 64, 5104.

(269) Merrifield, R. E. In Proceedings of 2nd International Symposium on Organic Solid State Chemistry, Rehovot, Israel, 1970; Butterworth: London, 1971; p 481.

(270) Yarmus, L.; Swenberg, C.; Rosenthal, J.; Arnold, S. Phys. Lett. 1973, 43A, 103.

(271) Pekcan, O. Chem. Phys. Lett. 1980, 70, 74

(272) Frankevich, E. L.; Rumyantsev, B. M.; Lesin, V. I. 6th Molecular Crystal Symposium, Schloss Elmau, FRG, 1973, unpublished.

(273) Okamoto, K.; Oda, N.; Itaya, A.; Kusbayashi, S. Chem. Phys. Lett. $1975,35,483$

(274) Frankevich, E. L.; Rumyantsev, B. M.; Lesin, V. I.; Kotov, B V. Opt. Spektrosk. 1977, 42, 905.

(275) Lesin, V. I.; Nikolaev, E. N.; Sakun, V. P. Opt. Spektrosk. $1981,50,1019$.

(276) Kaneto, K.; Yoshino, K.; Inuishi, Y. Chem. Phys. Lett. 1976 40,505 .

(277) Gliemann, G. Comments Inorg. Chem. 1986, 5, 263.

(278) Payne, S. A.; Austin, R. H.; McClure, D. S. Phys. Reu. B 1984 29,32 .
(279) Leung, M.; El-Sayed, M. A. J. Am. Chem. Soc. 1975, 97, 669. (280) Dellinger, B.; Hochstrasser, R. M.; Smith, A. B., III. J. Am. Chem. Soc. 1977, 99, 5834.

(281) Mori, K.; Tabata, Y. Kogyo Kagaku Zasshi 1970, 73, 1251.

(282) Mori, K.; Tabata, Y. Kogyo Kagaku Zasshi 1970, 73, 815

(283) Savel'ev, G. G.; Medvinskii, A. A.; Salikhov, K. M.; Sarvarov, F. S. Tezisy Dokl.-Vses. Konf. "Polyariz. Yader Elektronov Eff. Magn. Polya Khim. Reakts.”; Sagdeev, R. Z., Ed., 1975, p 41.

(284) Frankevich, E. L. Sou. Phys. JETP 1966, 23, 814.

(285) Ern, V;; Merrifield, R. E. Phys. Rev. Lett. 1968, 21, 609.

(286) Groff, R. P.; Merrifield, R. E.; Avakian, P.; Tomkiewicz, Y. Phys. Rev. Lett. 1970, 25, 105.

(287) Kotani, M. Chem. Phys. Lett. 1976, 43, 205

(288) Vankan, J. M. J.; Veeman, W. S. Chem. Phys. Lett. 1983, 94 , 133

(289) Groff, R. P.; Merrifield, R. E.; Avakian, P. Chem. Phys. Lett. $1970,5,168$.

(290) Podoplelov, A. V.; Leshina, T. V.; Sagdeev, R. Z.; Molin, Yu N. Dokl. Akad. Nauk SSSR 1976, 230, 150

(291) Leshina, T. V.; Maryasova, V. I.; Sagdeev, R. Z.; Margorskaya, O. I.; Bravo-Zhivotovskii, D. A.; Kruglaya, O. A.; Vyazankin, N. S. React. Kinet. Catal. Lett. 1979, 12, 491.

(292) Taraban, M. B.; Leshina, T. V.; Salikhov, K. M.; Sagdeev, R. Z.; Molin, Yu. N.; Margorskaya, O. I.; Vyazankin, N. S. $J$ Organomet. Chem. 1983, 256, 31 .

(293) Sagdeev, R. Z.; Molin, Yu. N.; Salikhov, K. M.; Leshina, T. V.; Kamha, M. A.; Shein, S. M. Org. Magn. Reson. 1973, 5, 599.

(294) Kubarev, S. I.; Pshenichov, E. A.; Shustov, A. S. Theor. Exp. Chem. 1979, 15, 10 .

(295) Turro, N. J.; Chow, M.-F.; Rigaudy, J. J. Am. Chem. Soc. $1981,103,7218$

(296) Tanimoto, Y.; Nishino, M.; Itoh, M. Bull. Chem. Soc. Jpn. $1985,58,3365$.

(297) Molin, Yu. N.; Sagdeev, R. Z.; Leshina, T. V.; Podoplelov, A. V.; Dushkin, A. V.; Grishin, Yu. A.; Weiner, L. M. In Mag. netic Resonance and Related Phenomena; Kundla, E., Lippmaa, E., Saluvere, T., Eds.; Springer: Berlin: 1979; p 49.

(298) Samarskaya, T. G.; Skrunts, L. K.; Kiprianova, L. A.; Levit, I. P.; Gragerov, I. P. Dokl. Phys. Chem. 1985, 283, 697.

(299) Broomhead, E. J.; McLauchlan, K. A. J. Chem. Soc., Faraday Trans. 2 1978, 74, 775

(300) Agulova, L. P.; Opalinskaya, A. M. Russ. J. Phys. Chem. (Engl. Transl.) 1985, 59, 890.

(301) Perito, P.; Corden, B. B. J. Am. Chem. Soc. 1987, $109,4418$.

(302) Schulten, K.; Staerk, H.; Weller, A.; Werner, H.-J.; Nickel, B. Z. Phys. Chem. (Frankfurt/Main) 1976, 101, 371.

(303) Werner, H.-J.; Staerk, H.; Weller, A. J. Chem. Phys. 1978, 68, 2419.

(304) Weller, A. Z. Phys. Chem. (Frankfurt/Main) 1982, 130, 129.

(305) Nolting, F.; Staerk, H.; Weller, A. Chem. Phys. Lett. 1982, 88, 523.

(306) Weller, A.; Nolting, F.; Staerk, H. Chem. Phys. Lett. 1983, 96, 24.

(307) Staerk, H.; Treichel, R.; Weller, A. Chem. Phys. Lett. 1983, $96,28$.

(308) Weller, A.; Staerk, H.; Treichel, R. J. Chem. Soc., Faraday Discuss. 1984, 78, 271, 332.

(309) Staerk, H.; Treichel, R.; Weller, A. In Biophysical Effects of Steady Fields; Maret, G., Kiepenheuer, J., Boccara, N., Eds.; Springer Proc. Phys. 1986, 11, 85.

(310) Leshina, T. V.; Salikhov, K. M.; Sagdeev, R. Z.; Belyaeva, S. G.; Maryasova, V. I.; Purtov, P. A.; Molin, Yu. N. Chem. Phys. Lett. 1980, 70, 228.

(311) Leshina, T. V.; Belyaeva, S. G.; Maryasova, V. I.; Sagdeev, R. Z.; Molin, Yu. N. Chem. Phys. Lett. 1980, 75, 438.

(312) Michel-Beyerle, M. E.; Krüger, H. W.; Haberkorn, R.; Seidlitz, H. Chem. Phys. 1979, 42, 441.

(313) Krüger, H. W.; Michel-Beyerle, M. E.; Seidlitz, H. Chem. Phys. Lett. 1982, 87, 79.

(314) Krüger, H. W.; Michel-Beyerle, M. E.; Knapp, E. W. Chem. Phys. 1983, 74, 205

(315) Periasamy, N.; Linschitz, H. Chem. Phys. Lett. 1979, 64, 281.

(316) Steiner, U. E. Z. Naturforsch. 1979, 34a, 1093.

(317) Nakagaki, R.; Hiramatsu, M.; Mutai, K.; Tanimoto, Y.; Nagakura, S. Chem. Phys. Lett. 1987, 134, 171.

(318) Hata, N.; Yamada, Y. Chem. Lett. 1980, 989.

(319) Hata, N.; Hokawa, M. Chem. Lett. 1981, 507.

(320) Hata, N.; Nishida, N. Chem. Lett. 1983, 1043.

(321) Tanimoto, Y.; Takashima, M.; Uehara, M.; Itoh, M.; Hiramatsu, M.; Nakagaki, R.; Watanabe, T.; Nagakura, S. Chem. Lett. 1985, 15 .

(322) Sakaguchi, Y.; Hayashi, H.; Nagakura, S. Bull. Chem. Soc. Jpn. 1980, 53,3059.

(323) Fischer, H. Chem. Phys. Lett. 1983, 100, 255

(324) Zimmt, M. B.; Doubleday, Ch., Jr.; Gould, I. R.; Turro, N. J. J. Am. Chem. Soc. 1985, 107, 6724.

(325) Hayashi, H.; Nagakura, S. Bull. Chem. Soc. Jpn. 1978, 51, 2862 . 
(326) Gupta, H.; Hammond, G. S. J. Chem. Phys. 1972, 57, 1789

(327) Hata, N. Bull. Chem. Soc. Jpn. 1985, 58, 1088.

(328) Schulten, K.; Weller, A. Biophys. J. 1978, 295.

(329) Closs, G. L.; Czerposki, M. S. J. Am. Chem. Soc. 1977, 99, 6127.

(330) Schulten, K. J. Chem. Soc. 1985, 82, 1312.

(331) Haberkorn, R. Chem. Phys. 1977, 24, 111

(332) Closs, G. L.; Czeropski, M. S. Chem. Phys. Lett. 1978, 53, 321

(333) Grellmann, K.-H.; Watkins, A. R.; Weller, A. J. Lumin. 1970 $1 / 2,678$.

(334) Steiner, U. E.; Winter, G.; Kramer, H. E. A. J. Phys. Chem. $1977,81,1104$.

(335) Steiner, U. E. Chem. Phys. Lett. 1980, 74, 108.

(336) Steiner, U. E.; Winter, G. Chem. Phys. Lett. 1978, 55, 364.

(337) Sakuragi, H.; Sakuragi, M.; Mishima, T.; Watanabe, S.; Hasegawa, M.: Tokumaru, K. Chem. Lett. 1975, 231.

(338) Atkins, P. W.; Evans, G. T. Mol. Phys. 1975, 29, 921.

(339) Borden, W. T., Ed. Diradicals; Wiley: New York, 1982.

(340) Salem, L.; Rowland, C. Angew. Chem., Int. Ed. Engl. 1972 11,92 .

(341) Closs, G. L.; Doubleday, C. E. J. Am. Chem. Soc. 1973, 95 , 2735.

(342) Chiu, C. C. Y. Ph.D. Thesis, Brown University, 1979.

(343) Doubleday, Ch., Jr. Chem. Phys. Lett. 1981, 77, 131.

(344) Doubleday, Ch., Jr. Chem. Phys. Lett. 1981, 81, 164.

(345) De Kanter, F. J. J.; Kaptein, R. J. Am. Chem. Soc. 1982, 104 4759 .

(346) De Kanter, F. J. J.; den Hollander, J. A.; Huizer, A. H.; Kaptein, R. Mol. Phys. 1977, 34, 857 .

(347) Bittl, R.; Treutlein, H.; Schulten, K. In Antennas and Reaction Centers of Photosynthetic Bacteria; Michel-Beyerle, M. E., Ed.; Springer Ser. Chem. Phys. 1985, 42, 264.

(348) Closs, G. L.: Miller, R. J.; Redwine, O. D. Acc. Chem. Res. $1985,18,196$.

(349) Zimmt, M. B.; Doubleday, Ch. Jr.; Turro, N. J. J. Am. Chem. Soc. $1985,107,6726$

(350) Zimmt, M. B.; Doubleday, Ch., Jr.; Turro, N. J. J. Am. Chem. Soc. $1986,108,3618$.

(351) Tanimoto, Y.; Okada, N.; Itoh, M.; Iwa, K.; Sugioka, K.; Takemura, F.; Nakagaki, R.; Nagakura, S. Chem. Phys. Lett. $1987,136,42$

(352) Tanimoto, Y.; Takeshima, M.; Hasegawa, K.; Itoh, M. Chem. Phys. Lett. 1987, 137, 330.

(353) Faulkner, L. R.; Bard, A. J. J. Am. Chem. Soc. 1969, 91, 209

(354) Faulkner, L. R.; Tachikawa, H.; Bard, A. J. J. Am. Chem. Soc. 1972, 94, 691 .

(355) Tachikawa, H, Bard, A. J. Chem. Phys, Lett. 1973, 19, 287.

(356) Petrov, N. Kh.; Fedotova, E. Ya.; Frankevich, E. L. High Energy Chem. 1980, 14, 257.

(357) Petrov, N. Kh.; Shushin, A. I.; Frankevich, E. L. Chem. Phys. Lett. $1981,82,339$

(358) Nath, D. N.; Chowdhury, M. Chem. Phys. Lett. 1984, 109, 13

(359) Faulkner, L. R.; Bard, A. J. J. Am. Chem. Soc. 1969, 91, 6495.

(360) Avakian, P.; Groff, R. P.; Kellogg, R. E.; Merrifield, R. E.; Suna, A. In Organic Scintillators and Liquid Scintillation Countings; Horrocks, D. L., Ed.; Academic: New York, 1971; p 499.

(361) Tachikawa, H.; Bard, A. J. Chem. Phys. Lett. 1974, 26, 246.

(362) Wyrsch, D.; Labhart, H. Chem. Phys. Lett. 1971, 8, 217.

(363) Spichtig, J.; Bulska, H.; Labhart, H. Chem. Phys. 1976, 15, 279.

(364) Van Willigen, H. Chem. Phys, Lett. 1975, 33, 540

(365) Tachikawa, H.; Bard, A. J. J. Am. Chem. Soc. 1973, 95, 1672

(366) Kearns, D. R.; Stone, A. J. J. Chem. Phys. 1971, 55, 3383

(367) Geacintov, N. E.; Swenberg, C. F. J. Chem. Phys. 1972, 57 , 378.

(368) Faulkner, L. R.; Bard, A. J. J. Am. Chem. Soc. 1969, 91, 6497.

(369) Tachikawa, H.; Bard, A. J. Chem. Phys. Lett. 1974, 26, 10

(370) Keszthelyi, C. P. Tokel-Takvoryan, N. E.; Tachikawa, H. Bard, A. J. Chem. Phys. Lett. 1973, 23, 219.

(371) Santhanam, K. S. V. Can. J. Chem. 1971, 49, 3577

(372) Periasamy, N.; Santhanam, K. S. V. Can. J. Chem. 1975, 53, 76.

(373) Vaz, F.; Santhanam, K. S. V. Indian J. Phys. 1977, 51B, 446

(374) Razi Naqvi, K.; Staerk, H.; Gillbro, T. Chem. Phys. Lett $1977,49,160$

(375) Periasamy, N.; Shah, S. J.; Santhanam, K. S. V. J. Chem. Phys. 1973, 58, 821.

(376) Emokhonov, V. N.; Tribel, M. M.; Klimov, A. L.; Kolesnikova, L. I. Khim. Fiz, $1984,3,12$

(377) Brocklehurst, B.; Dixon, R. S.; Gardy, E. M.; Lopata, V. J.; Quinn, M. J.; Singh, A.; Sargent, F. P. Chem. Phys. Lett. $1974,28,361$.

(378) Sargent, F. P.; Brocklehurst, B.; Dixon, R. S.; Gardy, E. M.; Lopata, V. J. Singh, A. J. Phys, Chem 1977, 81,815.

(379) Dixon, R. S.; Gardy, E. M.; Lopata, V. J.; Sargent, F. P. Chem. Phys. Lett. 1975, 30, 463.

(380) Dixon, R. S.; Sargent, F. P.; Lopata, V. J.; Gardy, E. M. Chem. Phys. Lett. 1977, 47, 108.

(381) Dixon, R. S.; Lopata, V. J. Can. J. Chem. 1979, 57, 3023.
(382) Anisimov, O. A.; Grigorijanz, B. M.; Kijanov, S. B.; Salikhov, K. M.; Suchenko, S. A.; Molin, Yu. N. Teor. Eksp. Khim. 1982, 18, 292.

(383) Dixon, R. S.; Sargent, F. P.; Lopata, V. J.; Gardy, E. M.; Brocklehurst, B. Can. J. Chem. 1977, 55, 2093.

(384) Brocklehurst, B. Chem. Phys. Lett. 1976, 44, 245.

(385) Appleton, W. R. S.; Brocklehurst, B. Chem. Phys. Lett. 1987, $136,199$.

(386) Anisimov, O. A.; Bizyaev, V. L.; Lukzen, N. N.; Grigoryants, V. M.; Molin, Yu. N. Dokl. Akad. Nauk SSSR 1983, 272, 383.

(387) Klein, J.; Voltz, R. Can. J. Chem. 1977, 55, 2102.

(388) Klein, J.: Voltz, R. Phys. Rev. Lett. 1976, 36, 1214

(389) Magee, J. L. In Comparative Effects of Radiation; Burton, M., Kirby-Smith, J. S., Magee, J. L., Eds.; Wiley: New York, $1960 ; p 130$.

(390) Mozumder, A.; Magee, J. L. Radiat. Res. 1966, 28, 203, 215.

(391) Brocklehurst, B. Chem. Phys. Lett. 1974, 28, 357.

(392) Magee, J. L.; Huang, J.-T. J. J. Phys. Chem. 1972, 76, 3801.

(393) Magee, J. L.; Huang, J.-T. J. J. Phys. Chem. 1974, 78, 310.

(394) Brocklehurst, B.; Higashimura, T. J. Phys. Chem. 1974, 78, 309.

(395) Atkins, P. W.; Lambert, T. P. Mol. Phys. 1976, 32, 1151.

(396) Brocklehurst, B. Nature (London) 1977, 265, 613.

(397) Brocklehurst, B. Nature (London) 1969, 221, 921.

(398) Hayashi, H.; Sakaguchi, Y.; Tsunooka, M.; Yanagi, H.; Tanaka, M. Chem. Phys. Lett. 1987, 136, 436 .

(399) Brocklehurst, B. J. Chem. Soc., Faraday Trans. 2 1979, 75 , 123.

(400) Winter, G.; Shiyoama, H.; Steiner, U. E. Chem. Phys. Lett. $1981,81,547$.

(401) Turro, N. J.; Cherry, W. R. J. Am. Chem. Soc. 1978, 100, 7431.

(402) Turro, N. J.; Kräutler, B. J. Am. Chem. Soc. 1978, 100, 7432.

(403) Hutton, R. S.; Roth, H. D.; Kräutler, B.; Cherry, W. R.; Turro, N. J. J. Am. Chem. Soc. 1979, 101, 2227.

(404) Turro, N. J.; Kräutler, B.; Anderson, D. R. J. Am. Chem. Soc. $1979,101,7435$.

(405) Kräutler, B.; Turro, N. J. Chem. Phys. Lett. 1980, 70, 270.

(406) Kräutler, B.; Turro, N. J. Chem. Phys. Lett. 1980, 70, 266.

(407) Turro, N. J.; Chow, M.-F.; Chung, C.-J.; Weed, G. C.; Kräutler, B. J. Am. Chem. Soc. 1980, 102, 4843.

(408) Turro, N. J.; Anderson, D. R.; Chow, M.-F.; Chung, C.-J.; Kräutler, B. J. Am. Chem. Soc. 1981, 103, 3892.

(409) Turro, N. J.; Mattay, J. Tetrahedron Lett. 1980, 21, 1799.

(410) Turro, N. J.; Mattay, J. J. Am. Chem. Soc. 1981, $103,4200$.

(411) Turro, N. J.; Chow, M.-F.; Cung, Ch.-J.; Tanimoto, Y.; Weed, G. C. J. Am. Chem. Soc. 1981, 103, 4574.

(412) Turro, N. J. Tetrahedron 1982, 38, 809

(413) Turro, N. J.; Chung, C.-J.; Lawler, R. G.; Smith, W. J. Tetrahedron Lett. 1982, $23,3223$.

(414) Turro, N. J.; Chow, M.-F.; Chung, Ch.-J.; Tung, Ch.-H. J. Am. Chem. Soc. 1983, 105, 1572

(415) Turro, N. J.; Zimmt, M. B.; Gould, I. R. J. Am. Chem. Soc. $1983,105,6347$

(416) Gould, I. R.; Tung, C.-H.; Turro, N. J.; Givens, R. S.; Matuszewski, B. J. Am. Chem. Soc. 1984, 106, 1789.

(417) Zimmt, M. B.; Doubleday, Ch., Jr.; Turro, N. J. J. Am. Chem. Soc. 1984, 106, 3363

(418) Turro, J. U.S. Patent 4448657 (Cl. 204-155; CO8F2/50), 1984.

(419) Gould, I. R.; Zimmt, M. B.; Turro, N. J.; Baretz, B. H.; Lehr, G. F. J Am. Chem. Soc. 1985, $107,4607$.

(420) Turro, N. J.; Lei, X.; Gould, I. R.; Zimmt, M. B. Chem. Phys. Lett. 1985, 120, 397 .

(421) Gould, I. R.; Baretz, B. H.; Turro, N. J. J. Phys. Chem. 1987, 91,925 .

(422) Sakaguchi, Y.; Nagakura, S.; Hayashi, H. Chem. Phys. Lett. $1980,72,420$.

(423) Sakaguchi, Y.; Nagakura, S.; Minoh, A.; Hayashi, H. Chem. Phys. Lett. $1981,82,213$.

(424) Sakaguchi, Y.; Hayashi, H.; Nagakura, S. J. Phys. Chem $1982,86,3177$.

(425) Sakaguchi, Y.: Hayashi, H. J. Phys. Chem. 1984, 88, 1437

(426) Hayashi, H.; Sakaguchi, Y.; Mochida, K. Chem. Lett. 1984 79.

(427) Hayashi, H.; Nagakura, S. Bull. Chem. Soc. Jpn. 1984, 57,

(428) Sakaguchi, Y.; Hayashi, H. Chem. Phys. Lett. 1984, 106, 420.

(429) Sakaguchi, Y.; Hayashi, H.; Murai, H.; I'Haya, Y. J. Chem. Phys. Lett. 1984, 110,275

(430) Sakaguchi, Y.; Hayashi, H.; Murai, H.; I'Haya, Y. J.; Mochida, K. Chem. Phys. Lett. 1985, 120, 401.

(431) Murai, H.; Sakaguchi, Y.; Hayashi, H.; I'Haya, Y. J. Phys. Chem. 1986, 90,113.

(432) Tanimoto, Y.; Itoh, M. Chem. Phys. Lett. 1981, 83, 1981.

(433) Tanimoto, Y.; Udagawa, H.; Itoh, M. J. Phys. Chem. 1983, 87,724 .

(434) Tanimoto, Y.; Shimizu, K.; Udagawa, H.; Itoh, M. Chem. Lett. 1983,353

(435) Tanimoto, Y.; Takashima, M.; Itoh, M. Bull. Chem. Soc. Jpn. 
$1984,57,1747$

(436) Tanimoto, Y.; Takashima, M.; Itoh, M. Chem. Phys. Lett. $1983,100,442$

(437) Tanimoto, Y;; Takashima, M.; Itoh, M. Chem. Lett. 1984, 1981.

(438) Tanimoto, Y.; Takayama, M.; Itoh, M.; Nakagaki, R.; Nagakura, S. Chem. Phys. Lett. 1986, 129, 414

(439) Schlenker, W.; Ulrich, T.; Steiner, U. E. Chem. Phys. Lett. $1983,103,118$

(440) Ulrich, T.; Steiner, U. E.; Schlenker, W. Tetrahedron 1986, 42,6131 .

(441) Baumann, D.; Ulrich, T.; Steiner, U. E. Chem. Phys. Lett. $1987,137,113$

(442) Scaiano, J. C.; Abuin, E. B. Chem. Phys. Lett. 1981, 81, 209.

(443) Scaiano, J. C.; Abuin, E. B.; Stewart, L. C. J. Am. Chem. Soc. $1982,104,5673$

(444) Scaiano, J. C.; Lougnot, D. J. J. Phys. Chem. 1984, 88, 3379.

(445) Closs, G. L.; Forbes, M. D. E.; Norris, J. R., Jr. J. Phys. Chem. 1987, 91, 3592 .

(446) Buckley, C. D.; Hunter, D. A.; Hore, P. J.; McLauchlan, K. A. Chem. Phys. Lett. 1987, 135, 307 .

(447) Thurnauer, M. C.; Meisel, D. J. Am. Chem. Soc. 1983, 105, 3729.

(448) Fendler, J. H. CHEMTECH 1985, 686

(449) Levin, P. P.; Kuz'min, V. A. Izv. Akad. Nauk SSSR, Ser. Khim. 1986, 35, 464.

(450) Levin, P. P.; Darmanyan, A. P.; Kuz'min, V. A.; Yankelevich, A. Z.; Kuznets, V. M. Izv. Akad. Nauk SSSR, Ser. Khim. $1980,12,2744$

(451) Trifunac, A. D.; Nelson, D. J. Chem, Phys. Lett, 1977, 46, 346.

(452) Tsunooka, M.; Tanaka, S.; Tanaka, M. Makromol. Chem. Rapid Commun. 1983, 4, 539 .

(453) Imoto, M.; Nomoto, K. Makromol. Chem., Rapid Commun. $1981,2,703$.

(454) Eicke, H. F.; Geiger, S.; Sauer, F. A.; Thomas, H. Ber. Bunsenges Phys. Chem. 1986, 90, 872 .

(455) Atkins, P. W.; Kivelson, D. J. Chem. Phys. 1966, 44, 169.

(456) Suna, A. Phys. Rev. B 1970, 1, 1716.

(457) Deutch, J. M. J. Chem. Phys. 1972, 56, 6076

(458) Watanabe, T.; Tanimoto, Y.; Sakata, T.; Nagagaki, R.; Hiramatsu, M.; Nagakura, S. Bull. Chem. Soc. Jpn. 1985, 58, 1251.

(459) Turro, N. J.; Cheng, C.-C.; Mahler, W. J. Am. Chem. Soc. 1984, 106, 5022

(460) Turro, N. J.; Cheng, C.-C.; Wan, P.; Chung, C.-J.; Mahler, W. J. Phys. Chem. 1985, 89, 1567.

(461) Frankevich, E. L.; Rumyantsev, B. M. Sov. Phys. JETP 1973, 36,1064

(462) Frankevich, E. L. Sokolik I A. JETP Lett. 1971, 14, 401.

(463) Frankevich, E. L.; Tribel, M. M.; Sokolik, I. A. Phys. Status Solidi $B$ : $1976, B 77,265$.

(464) Nickel, B.; Staerk, H.; Weller, A. Chem. Phys. Lett. 1969, 4, 27.

(465) Groff, R. P.; Merrifield, R. E.; Suna, A.; Avakian, P. Phys. Rev. Lett. 1972, 29, 429

(466) Groff, R. P. Suna, A.; Avakian, P.; Merrifield, R. E. Phys. Rev. B 1974, 9, 2655

(467) Papier, G.; Charle, K.-P.; Willig, F. Ber. Bunsenges. Phys Chem. 1982, 86, 670

(468) Farkas, L.; Sachsse, H. Z. Phys. Chem. B 1933, 23, 1.

(469) Farkas, L.; Sachsse, H. Z. Phys. Chem. B 1933, 23, 19

(470) Sachsse, H. Z. Phys. Chem. B 1934, 24, 429

(471) Wilmarth, W. K.; Baes, C. F., Jr. J. Chem. Phys. 1952, 20 , 116.

(472) Schwab, G.-M.; Voitländer, J. Z. Phys. Chem. (Frankfurt/ Main) 1955, 3, 341.

(473) Schwab, G.-M.; Voitländer, J.; Penka, V. Z. Phys. Chem. (Frankfurt/Main) 1963, 36, 378.

(474) Selwood, P. W. L. J. Catal. 1970, 19, 353

(475) Eley, D. D.; Forrest, H.; Pearce, D. R.; Rudham, R. J. Chem Soc, Chem Commun. 1972, 1176.

(476) Arias, J. A.; Selwood, P. W. J. Catal. 1974, 33, 284.

(477) Ashmead, D. R.; Eley, D. D.; Rudham, R. J. Catal. 1964, 3,

(478) Ng, C. F. Selwood, P. W. J. Catal. 1976, 43, 252.

(479) Arias, J. A.; Selwood, P. W. J. Catal. 1974, 35, 273.

(480) Misono, M.; Selwood, P. W. J. Am. Chem. Soc. 1968, 90 2977.

(481) Steiner, U. E.; Wolff, H.-J.; Ulrich, T.; Ohno, T., to be published.

(482) Blankenship, R. E.; Schaafsma, T. J.; Parson, W. W. Biochim. Biophys. Acta 1977, 461,297.

(483) Hoff, A. J.; Rademaker, H.; van Grondelle, R.; Duysens, L N. M. Biochim. Biophys. Acta 1977, 460, 547.

(484) Gorter de Vries, H.; Hoff, A. J. Chem. Phys. Lett. 1978, 55, 395.

(485) Rademaker, H.; Hoff, A. J.; Duysens, L. N. M. Biochim. Biophys. Acta 1979, 546, 248.

(486) Klevanik, A. V.; Shuvalov, V. A.; Goryushikin, G. E. Dokl. Akad. Nauk SSSR 1979, 249, 1238.
(487) Voznyak, V. M.; Elfimov, E. 1.; Proskuryakov, I. I. Dokl. Akad. Nauk SSSR 1978, 242, 1200 .

(488) Chidsey, C. E. D.; Roelofs, M. G.; Boxer, S. C. Chem. Phys. Lett. 1980, 74, 113 .

(489) Michel-Beyerle, M. E.; Scheer, H.; Seidlitz, H.; Tempus, D. FEBS Lett. 1980, 110, 129.

(490) Rademaker, H.; Hoff, A. J.; van Grondelle, R.; Duysens, L. N. M. Biochim. Biophys. Acta 1980, 592, 240 .

(491) Rademaker, H. Ph.D. Dissertation, University of Leiden, 1980.

(492) Sonneveld, A.; Duysens, L. N. M.; Moedijk, A. Proc. Natl. Acad. Sci. U.S.A. 1980, 77, 5889.

(493) Voznyak, V. M.; Elfimov, E. I.; Sukuvatitzina, V. K. Biochim. Biophys. Acta 1980, 592, 235.

(494) Voznyak, V. M.; Ganago, I. B.; Moskalenko, A. A.; Elfimov, E. I. Biochim. Biophys. Acta 1980,592, 364

(495) Norris, J. R.; Bowman, M. K.; Closs, G. L.; Budil, D. E., private communication, ref 37.

(496) Van Bochove, A. C.; van Grondelle, R.; Duysens, L. N. M. In Structure and Mechanism of the Photosynthetic Apparatus (Photosynthesis, Vol. 3); Akoyunoglou, G., Ed.; International Science Services: Philadelphia, 1981 p 989.

(497) Proskuryakov, I. I.; Elfimov, E. I.; Voznyak, V. M. Stud. Biophys. 1981, 84, 125 .

(498) Tribel, M. M.; Frankevich, E. L.; Kolesnikova, N. I. Abstracts of Papers, Conference on Chemically Induced Spin Polarization and Magnetic Field Effects in Chemical Reactions, Novosibirsk, SSSR, 1981, p 119.

(499) Sonneveld, A.; Duysens, L. N. M.; Moerdijk, A. Biochim. Biophys. Acta 1981, 636, 39

(500) Schenck, C. C.; Blankenship, R. E.; Parson, W. W. Biochim. Biophys. Acta 1982, 680, 44.

(501) Ogrodnik, A.; Krüger, H. W.; Orthuber, H.; Haberkorn, R.; Michel-Beyerle, M. E. Biophys. J. 1982, 39, 91.

(502) Roelofs, M. G.; Chidsey, C. E. D.; Boxer, S. G. Chem. Phys. Lett. $1982,87,582$.

(503) Boxer, S. G.; Chidsey, C. E. D.; Roelofs, M. G. J. Am. Chem. Soc. $1982,104,1452$.

(504) Boxer, S. G.; Chidsey, C. E. D.; Roelofs, M. G. J. Am. Chem. Soc. $1982,104,2674$.

(505) Boxer, S. G.: Chidsey, C. E. D.; Roelofs, M. G. Proc. Natl. Acad. Sci. U.S.A. 1982, 79, 4632.

(506) Norris, J. R.; Bowman, M. K.; Budil, D. E.; Tang, J.; Wraight, C. A.; Closs, G. L. Proc. Natl. Acad. Sci. U.S.A. 1982, 79, 5532

(507) Wasielewski, M. R.; Bock, Ch. H.; Bowman, M. K.; Norris, J. R. J. Am. Chem. Soc. 1983, 105, 2903.

(508) Wasielewski, M. R.; Bock, Ch. H.; Bowman, M. K.; Norris, J. R. Nature (London) 1983, 303,520.

(509) Chidsey, C. E. D.; Kirmaier, Ch.: Holten, D.; Boxer, S. G. Biochim. Biophys. Acta 1984, 766, 424.

(510) Boxer, S. G. In Antennas and Reaction Centers of Photosynthetic Bacteria (Springer Ser. Chem. Phys. Vol. 42); Michel-Beyerle, M. E., Ed.; Springer: Berlin, 1985; p 306.

(511) Chidsey, C. E. D.; Takiff, L.; Goldstein, R. A.; Boxer, S. G. Proc. Natl. Acad. Sci. U.S.A. 1985, 82,6850.

(512) Norris, J. R.; Budil, D. E.; Kolaczkowski, S. V.; Tang, J. H.; Bowman, M. K. In Antennas and Reaction Centers of Photosynthetic Bacteria (Springer Ser. Chem. Phys., Vol. 42); Michel-Beyerle, M. E., Ed.; Springer: Berlin, 1985; p 190.

(513) Lous, E. J.; Hoff, A. J. Photosynth. Res. 1986, 89

(514) Hoff, A. J.; Lous, E. J. In Biophysical Effects of Steady Fields (Springer Proc. Phys., Vol. 11); Maret, G., Kiepenheuer, J., Boccara, N., Eds.; Springer: Berlin, 1986; p 74.

(515) Ogrodnik, A.; Remy-Richter, N.; Michel-Beyerle, M. E. Chem. Phys. Lett. 1987, 135, 576.

(516) Williamson, S. J.: Romani, G.-L.; Kaufman, L.; Modenea, I., Eds. Biomagnetism; Plenum: New York, 1983

(517) Dubrov, A. P. The Geomagnetic Field and Life; Plenum: London, 1978

(518) Barnothy, M. F. Ed. Biological Effects of Magnetic Fields; Plenum: New York, 1965 (Vol. 1), 1969 (Vol. 2).

(519) Davis, A. R.; Rawls, W. C., Jr. The Magnetic Effect; Exposition Press: Hicksville, NY, 1975.

(520) Davis, A. R.; Rawls, W. C., Jr. Magnetism and Its Effect on the Living System; Exposition Press: Hicksville, NY, 1974

(521) Tenforde, T. S., Ed. Magnetic Field Effect on Biological Systems; Plenum: New York, 1979.

(522) Schulten, K.; Swenberg, Ch. E.; Weller, A. Z. Phys. Chem. (Frankfurt/Main) 1978, 111,

(523) Schulten, K.; Windemuth, A. In Biophysical Effects of Steady Fields (Springer Proc. Phys., Vol. 11); Maret, G., Kiepenheuer, J,, Boccara, N., Eds.; Springer: Berlin, 1986; p 99 .

(524) Gerstman, B.; Austin, R. H.; Hopfield, J. J. Phys. Rev. Lett. $1981,47,1636$.

(525) Redi, M.' H.; Gerstman, B. S.; Hopfield, J. J. Biophys. J. 1981 $35,471$.

(526) Doetschman, D. C.; Szumowski, J. J. Chem. Phys. 1986, 84, 2866 
(527) Martin, J.-L.; Breton, J.; Hoff, A. J.; Migus, A.; Antonetti, A. Proc. Natl. Acad. Sci. U.S.A. 1986, 83,957.

(528) Deisenhofer, J.; Epp, O.; Miki, K.; Huber, R.; Michel, H. J. Mol. Biol. 1984, 180, 385 .

(529) Deisenhofer, J.; Epp, O.; Miki, K.; Huber, R.; Michel, H. Nature (London) 1985, 318, 618.

(530) Hoff, A. J. In Triplet State ODMR Spectroscopy; Clarke, R. H., Ed.; Wiley: New York, 1982; p 367.

(531) Hoff, A. J. In Light Reaction Paths in Photosynthesis (Mol. Biol., Biochem. Biophys., Vol. 35); Fong, F. K., Ed.; Springer: Berlin, 1982; pp 80, 323.

(532) Werner, H. J.; Schulten, K.; Weller, A. Biochim. Biophys. Acta 1978, 502, 255

(533) Haberkorn, R.; Michel-Beyerle, M. E. Biophys. J. 1979, 26, 489.

(534) Haberkorn, R.; Michel-Beyerle, M. E.; Marcus, R. A. Proc. Natl. Acad. Sci. U.S.A. 1979, 76, 4185.

(535) Rademaker, H.; Hoff, A. J. Biophys. J. 1981, 34, 325

(536) Ogrodnik, A.; Lersch, W.; Michel-Beyerle, M. E. In Antennas and Reaction Centers of Photosynthetic Bacteria (Springer Ser. Chem. Phys., Vol. 42); Michel-Beyerle, M. E., Ed.; Springer: Berlin, 1985; $\mathrm{p} 198$.

(537) Hore, P. J.; Hunter, D. A.; McKie, C. D.; Hoff, A. J. Chem. Phys. Lett. 1987, 137, 495.

(538) Frankevich, E. L.; Pristupa, A. I. Pisma Zh. Eksp. Teor. Fiz. $1976,24,397$.

(539) Frankevich, E. L.; Pristupa, A. I.; Tribel, M. M.; Sokolik, I. A. Dokl. Akad. Nauk SSSR 1977, 236, 1173

(540) Frankevich, E. L.; Lesin, V. I.; Pristupa, A. I. Chem. Phys. Lett. 1978, 58, 127.

(541) Frankevich, E. L.; Tribel, M. M.; Sokolik, I. A.; Pristupa, A. I. Phys. Status Solidi B: 1978, B87, 373.

(542) Lesin, V. I.; Sakun, V. P.; Pristupa, A. I.; Frankevich, E. L. Phys. Status Solidi B: 1977, B84, 513.

(543) Frankevich, E. L.; Pristupa, A. I.; Lesin, V. I. Pisma $Z h$. Eksp. Teor. Fiz. 1977, 26, 725.

(544) Frankevich, E. L.; Pristupa, A. I.; Lesin, V. I. Chem. Phys. Lett. 1978, 54, 99

(545) Frankevich, E. L. Lesin, V. I.; Pristupa, A. I. In Magnetic Resonance and Related Phenomena; Kundla, E., Lippmaa, E., Saluvere, T., Eds.; Springer: Berlin, 1979; p 45

(546) Steudle, W.; von Schütz, J. U. J. Lumin. 1979, 18/19, 191.

(547) Frankevich, E. L.; Lesin, V. I.; Pristupa, A. I. Sov. Phys. JETP (Engl. Transl.) 1978, 48, 208; Zh. Eksp. Teor. Fiz. $1978,75,415$.

(548) Anisimov, O. A.; Grigoryants, V. I.; Molin, Yu. N. Dokl. Akad. Nauk SSSR 1979, 248, 380.

(549) Anisimov, O. A.; Grigoryants, V. I.; Molin, Yu. N. Prisma Zh. Eksp. Teor. Fiz. 1979, 30,589.

(550) Smith, J. P.; Trifunac, A. D. J. Phys. Chem. 1981, 85, 1645.

(551) Smith, J. P.; Trifunac, A. D. Chem. Phys. Lett. 1981, 83, 195.

(552) Anisimov, O. A.; Grigoryants, V. I.; Molin, Yu. N. Chem. Phys. Lett. 1980, 74, 15

(553) Molin, Yu. N.; Anisimov, O. A. Radiat. Phys. Chem. 1983, 21, 77.

(554) Melekhov, V. I.; Anisimov, O. A.; Saik, V. O.; Molin, Yu. N. Chem. Phys. Lett. 1984, 112, 106.

(555) Molin, Yu. N.; Anisimov, O. A.; Melekhov, V. I.; Smirnov, S. N. J. Chem. Soc., Faraday Discuss. 1984, 78, 289.

(556) Lefkowitz, S. M.; Trifunac, A. D. J. Phys. Chem. 1984, 88, 77.

(557) Smirnov, S. N.; Rogov, V. A.; Shustov, A. S.; Sheberstov, S. V.; Panfilovitch, N. V.; Anisimov, O. A.; Molin, Yu. N. Chem. Phys. 1985, 92, 381.

(558) Series, G. W. In Quantum Optics; Kay, S. M., Mailland, A., Eds.; Academic: London, 1979; p 395.

(559) Tarasov, V. F.; Buchachenko, A. L.; Maltsev, V. I. Zh. Fiz. Khim. 1981, 55, 1921.

(560) Yurke, B.; Denker, J. S.; Johnson, B. R.; Bigelow, N.; Levy, L. P.; Lee, D. M.; Freed, J. H. Phys. Rev. Lett. 1983, 50, 1137.

(561) El-Sayed, M. A. Adv. Photochem. 1974, 9, 311.

(562) Hausser, K. H.; Wolff, H. C. Adv. Magn. Reson. 1976, 8, 85

(563) Kinoshita, M.; Iwasaka, N.; Nishi, N. Appl. Spectrosc. Rev. $1981,17,1$

(564) Grundler, W.; Keilmann, F.; Putterlik, V.; Santo, L.; Strube, D.; Zimmermann, I. In Coherent Excitations in Biologica Systems; Fröhlich, H., Kremer, F., Eds.; Springer: Berlin, 1983; p 21

(565) Grundler, W.; Keilmann, F. Phys. Rev. Lett. 1983, 51, 1214.

(566) Keilmann, F. Phys. Z. 1985, 16, 33.

(567) Kubarev, S. I.; Pshenichnov, E. A. Chem. Phys. Lett. 1974, 28,66 .

(568) Lepine, D. J. Phys. Rev. 1972, B6, 436.

(569) Morigaki, K.; Dunsten, D. J.; Cavenett, B. C.; Dawson, P.; Nicholls, J. E.; Nitta, S.; Shimakawa, K. Solid State Commun. $1978,26,981$

(570) Street, R. A. Phys. Rev. 1982, B26, 3588.

(571) Davis, J. J.; Nichols, J. E.; Cavenett, B. C. Semiconductors Insulators 1978, $4,101$.

(572) Cox, R. T.; Block, D.; Herve, A.; Picard, R.; Santier, C.; Helbig, R. Solid State Commun. 1978, 25, 77.
(573) Kaplan, D.; Solomon, I.; Mott, N. F. J. Phys., Lett. 1978, 39, 51

(574) Haberkorn, R.; Dietz, W. Solid State Commun. 1980, 35, 505

(575) Dawson, P.; Cavenett, B. C.; Sowersby, G. Solid-State Electron. 1978, 21, 1451.

(576) Tarasov, V. F.; Buchachenko, A. L. Izv. Akad. Nauk SSSR, Ser. Khim. 1980, 8, 1927.

(577) Sterna, L.; Ronis, D.; Wolfe, S.; Pines, A. J. Chem. Phys. $1980,73,5493$

(578) Turro, N. J.; Anderson, D. R.; Kräutler, B. Tetrahedron Lett. $1980,21,3$.

(579) Tarasov, V. F.; Askerov, D. B.; Buchachenko, A. L. Bull. Acad. Sci. USSR, Div. Chem. Sci. 1982, 31, 1786.

(580) Klimenko, B. B.; Tarasov, V. F.; Buchachenko, A. L. Bull. Acad. Sci. USSR, Div. Chem. Sci. 1984, 33, 1072

(581) Churchwell, E.; Walmsley, C. M.; Winnewisser, G. Astron. Astrophys. 1977, 54, 925.

(582) Haberkorn, R.; Michel-Beyerle, M. E.; Michel, K. W. Astron. Astrophys. 1977, 55, 315.

(583) Bube, W. Doctoral Thesis (English), Technische Universität München, 1977.

(584) Belyakov, V. A.; Mal'tsev, V. I.; Galimov, E. M.; Buchachenko, A. L. Dokl. Akad. Nauk SSSR 1979, 243, 561 .

(585) Buchachenkov, A. L.; Belyakov, V. A.; Mal'tsev, V. I. Bull. Acad. Sci. USSR, Div. Chem. Sci. 1982, 31,906.

(586) Buchachenko, A. L.; Yasina, L. L.; Makhov, S. F.; Mal'tsev V. I.; Fedorov, A. V.; Galimov, E. M. Dokl. Akad. Nauk SSSR 1980,260, 431 .

(587) Buchachenko, A. L.; Belyakov, V. A.; Mal'tsev, V. I. Bull. Acad. Sci. USSR, Div. Chem. Sci. 1982, 31,900.

(588) Buchachenko, A. L.; Fedorov, A. V.; Yasina, L. L. Chem. Phys. Lett. 1984, 103, 405.

(589) Buchachenko, A. L. Russ. J. Phys. Chem. (Engl. Transl.) $1977,51,1445$.

(590) Buchachenko, A. L. Russ. J. Phys. Chem. (Engl. Transl.) $1981,55,936$

(591) Lawler, R. G. J. Am. Chem. Soc. 1980, 102, 430

(592) Clayton, R. N.; Grossman, L.; Mayeda, T. K. Science (Washington, D.C.) $1973,182,485$.

(593) Bernstein, R. J. Phys. Chem. 1952, 56, 893.

(594) Tarasov, V. F. Russ. J. Phys. Chem. (Engl. Transl.) 1980, 54, 1391.

(595) Podoplelov, A. V.; Leshina, T. V.; Sagdeev, R. Z.; Molin, Yu. N.; Gol'danskii, V. I. JETP Lett. 1979, 29, 380 .

(596) Podoplelov, A. V.; Medvedev, V. I.; Sagdeev, R. Z.; Salikhov, K. M.; Molin, Yu. N. Abstracts of Papers, Conference on Chemically Induced Spin Polarization and Magnetic Effects in Chemical Reactions, Novosibirsk, 1981, p 81.

(597) Gelbart, W. M.; Freed, K. F. Chem. Phys. Lett. 1973, 18, 470.

(598) Freed, K. F.; Tric, C. Chem. Phys. 1978, 33, 249.

(599) Freed, K. F. Adv. Chem. Phys. 1980, 42, 207.

(600) Stannard, P. R. J. Chem. Phys. 1978, 68, 3932.

(601) de Kronig, R. L. Z. Phys. 1930, 62, 300

(602) Minaev, B. F. Opt. Spectrosc. (USSR) 1978, 44, 148.

(603) Steinfeld, J. I.; Zare, R. N.; Jones, L.; Lesk, M.; Klemperer, W. J. Chem. Phys. 1965, 42, 25.

(604) Tellinghuisen, J. J. Chem. Phys. 1973, 58, 2821

(605) Schlag, E. W. J. Photochem. 1978, 9, 97.

(606) Selzle, H. L.; Lin, S. I.; Schlag, E. W. Chem. Phys. Lett. 1979, $62,230$.

(607) Grimbert, D.; Lavollee, M.; Nitzan, A.; Tramer, A. Chem. Phys. Lett. 1978, 57, 45.

(608) El-Sayed, M. A.; Leyerle, R. J. Chem. Phys. 1975, 62, 1579.

(609) Atkins, P. W.; Evans, G. T. Mol. Phys. 1974, 27, 1633.

(610) Atkins, P. W.; Topping, G. S. Mol. Phys. 1982, 46, 941

(611) Pedersen, J. B.; Freed, J. H. J. Chem. Phys. 1975, 62, 1706

(612) Serebrennikov, Yu. A.; Minaev, B. F. Chem. Phys. 1987, 114, 359

(613) Hore, P. J. Chem. Phys. Lett. 1980, 69, 563

(614) Felix, C. C.; Weissman, S. I. Proc. Natl. Acad. Sci. U.S.A. $1975,72,4203$

(615) Grant, A. I.; McLauchlan, K. A. Chem. Phys. Lett. 1983, 101, 120.

(616) McLauchlan, K. A. In ref 23, p 151

(617) Atkins, P. W. In ref 23, p 191 .

(618) Clarke, R. H.; Connors, R. E.; Keegan, J. J. Chem. Phys. $1977,66,358$.

(619) Gehrtz, M.; Bräuchle, Ch.; Voitländer, J. Z. Naturforsch. $1981,36 a, 743$

(620) Fano, U.; Racah, G. Irreducible Tensorial Sets; Academic: New York, 1959 .

(621) Edmonds, A. R. Angular Momentum in Quantum Mechanics; Princeton University Press: Princeton, NJ, 1957.

(622) Rose, M. E. Elementary Theory of Angular Momentum; Wiley: New York, 1967.

(623) Freed, J. H.; Bruno, G. V.; Polnaszek, C. J. Chem. Phys. 1971, 55,5270 .

(624) Gupta, A. Doctoral Thesis, California Institute of Technology, Pasadena, CA, 1974.

(625) Deutsch, M.; Brown, S. C. Phys. Rev. 1952, 85, 1047. 
(626) Halpern, O. Phys. Rev. 1954, 94, 904.

(627) Hughes, V. W.; Marder, S.; Wu, C. S. Phys. Rev. 1955, 98 1840.

(628) Rusin, B. A.; Frankevich, E. L. Phys. Status Solidi 1969, 33, 885.

(629) Bargon, J.; Fischer, H. Z. Naturforsch. 1967, 22a, 1556.

(630) Lawler, R. G. J. Am. Chem. Soc. 1967, 89, 5519.

(631) Kaptein, R.; Oosterhoff, J. L. Chem. Phys. Lett. 1969, 4, 214.

(632) Closs, G. L. J. Am. Chem. Soc. 1969, 91, 4552

(633) Gerhart, F. Tetrahedron Lett. 1969, 53, 5061.

(634) Closs, G. L.; Trifunac, A. D. J. Am. Chem. Soc. 1970, 92, 2183.

(635) Adrian, F. J. J. Chem. Phys. 1970, 53, 3374

(636) Adrian, F. J. J. Chem. Phys. 1971, 54, 3912.

(637) Adrian, F. J. J. Chem. Phys. 1971, 54, 3918

(638) Noyes, R. M. J. Chem. Phys. 1954, 22, 1349.

(639) Sagdeev, R. Z.; Salikhov, K. M.; Leshina, T. V.; Kamkha, M. A.; Shein, S. M.; Molin, Yu. N. JETP Lett. 1972, 16, 422.

(640) Kubo, R. J. Phys. Soc. Jpn. 1969, 26 (Suppl.), 1.

(641) Lersch, W.; Michel-Beyerle, M. E. Chem. Phys. Lett. 1984, 107,522 .

(642) Salikhov, K. M. Chem. Phys. 1983, 82, 163.

(643) Werner, H.-J.; Schulten, Z.; Schulten, K. J. Chem. Phys. $1977,67,646$.

(644) Reference 52, p 65

(645) Reference 52, 78 .

(646) Salikhov, K. M. Chem. Phys. 1983, 82, 145.

(647) Brocklehurst, B. J. Chem. Soc., Faraday Trans. 2 1976, 72, 1869 .

(648) Evans, G. T.; Lawler, R. G. Mol. Phys. 1975, 30, 1085

(649) Schulten, Z.; Schulten, K. J. Chem. Phys. 1977, 66, 4616.

(650) Reference 52 , p 66.

(651) Knapp, E.-W.; Schulten, K. J. Chem. Phys, 1979, 71, 1878.

(652) Purtov, P. A.; Salikhov, K. M. Theor. Exp. Chem. 1980, 16 413.

(653) Polyakov, N. E.; Purtov, P. A.; Leshina, T. V.; Taraban, M. B.; Sagdeev, R. Z.; Salikhov, K. M. Chem. Phys. Lett. 1986, $129,357$.

(654) Polyakov, N. E,; Taraban, M. B.; Leshina, T. V.; Salikhov, K. M.; Sagdeev, R. Z. Chem. Phys. Lett. 1987, 136, 31.

(655) Kubarev, S. I.; Pshenichov, E. A.; Shustov, A. S. Theor. Exp. Chem. 1976, 12,335 .

(656) Salikhov, K. M. Theor. Eksp. Khim. 1977, 13, 732.

(657) Pedersen, J. B. J. Chem. Phys. 1977, 67, 4097.

(658) Purtov, P. A.; Salikhov, K. M. Theor. Exp. Chem. 1980, 16, 530 .

(659) Fischer, H. Z, Naturforsch. 1970, 25a, 1957.

(660) Kaptein, R. J. Am. Chem. Soc. 1972, 94, 6251.

(661) Salikhov, K. M. Doctoral Thesis, Kazan, USSR, 1974

(662) Adrian, J. J. Chem. Phys. 1972, 57, 5107.

(663) Atkins, P. W. Moore, E. A. Mol. Phys, 1973, 25, 825.

(664) Atkins, P. W.; Gurd, R. C. Chem. Phys. Lett. 1972, 16, 265.

(665) Bittl, R.; Schulten, K. In Biophysical Effects of Steady Fields (Springer Proc. Phys., Vol. 11); Maret, G., Kiepenheuer, J., Boccara, N., Eds.; Springer: Berlin, 1986; p 90.

(666) Kubo, R. Adv. Chem. Phys. 1969, 15, 101.

(667) Kaptein, R. J. Am. Chem. Soc. 1972, 94, 6262.

(668) Sarvarov, F. S.; Kobzareva, B. A.; Shmidt, V. N.; Salikhov K. M. Zh. Fiz. Khim. 1982, 56, 1585

(669) Reference 52, p 89.

(670) Hore, P. J.; McLauchlan, K. A. Mol. Phys. 1981, 42, 533.

(671) Salikhov, K. M.; Sarvarov, F. S. Theor. Exp. Chem. 1982, 18, 124.

(672) Kruppa, A. I.; Leshina, T. V.; Sagdeev, R. Z.; Salikhov, K. M.; Sarvarov, F. S. Chem. Phys. 1982, 67, 1329

(673) Knapp, E. W.; Lersch, W. Chem. Phys. Lett. 1984, 110, 259

(674) Schulten, K. J. Chem. Phys. 1984, 80, 3668

(675) Closs, G. L.; Doubleday, C. E.; Paulson, D. R. J. Am. Chem. Soc. 1970, 92, 2185

(676) Atkins, P. W. Chem. Phys. Lett. 1973, 18, 355.

(677) Hatlee, M. D.; Kozak, J. J.; Rothenberger, G.; Infelta, P. P.; Grätzel, M. J. Phys. Chem. 1980, 84, 1508 .

(678) Gösele, U.; Klein, U. K. A.; Hauser, M. Chem. Phys, Lett. $1979,68,291$.

(679) Buchachenko, A. L.; Markarian, Sh. A. Org. Magn. Reson. $1973,5,247$.

(680) Purtov, P. A.; Salikhov, K. M. Theor. Exp. Chem. 1979, 15, 90.

(681) Kaptein, R.; den Hollander, J. A. J. Am. Chem. Soc. 1972, 94, 6269

(682) Haberkorn, R. Chem. Phys. 1977, 19, 165

(683) Abell, G. C. Mozumder, A. 1972, 56, 4079

(684) Razi Naqui, K.; Mork, K. J.; Waldenström, S. J. Phys. Chem. $1980,84,1315$

(685) Tomkiewicz, M.; Cocivera, M. Chem. Phys. Lett. 1971, 8, 595.

(686) Buchachenko, A. L.; Markarian, Sh. A. React. Kinet. Catal. Lett. $1974,1,157$

(687) Sarvarov, F. S.; Salikhov, K. M. Theor, Exp. Chem. 1976, 12, 365 .

(688) Haberkorn, R.; Michel-Beyerle, M. E. Z. Naturforsch. 1976 $31 a, 499$.
(689) Atkins, P. W. In ref $23, \mathrm{p} 383$

(690) Salikhov, K. M. Theor. Exp. Chem. 1977, 13, 551.

(691) Brocklehurst, B. J. Chem. Soc., Faraday Trans. 2 1982, 78, 751.

(692) Brocklehurst, B. Radiat. Phys. Chem. 1984, 23, 187

(693) Brocklehurst, B. J. Chem. Soc., Faraday Discuss. 1984, 78, 303

(694) Belyakov, V. A.; Buchachenko, A. L. Chem. Phys. (Russ.) $1983,10,1385 ; 11,1510$

(695) Schulten, K.; Bittl, R. J. Chem. Phys. 1986, 84, 5155.

(696) Bloch, R. Phys. Rev. 1957, 105, 1206.

(697) Fano, U. Rev. Mod. Phys. 1957, 29, 74

(698) Kaplan, J. J. Chem. Phys. 1958, 28, 278; 29, 462

(699) Tomkiewicz, M.; Groen, A.; Cocivera, M. J. Chem. Phys. $1972,56,5850$

(700) Evans, G. T.; Fleming, P. D., III; Lawler, R. G. J. Chem. Phys. 1973, 58, 2071.

(701) Pedersen, J. B.; Freed, J. H. J. Chem. Phys. 1973, 59, 2869

(702) Haberkorn, R. Mol. Phys, 1976, 32, 1491.

(703) Atkins, P. W.; Evans, G. T. Chem. Phys. Lett. 1974, 24, 45.

(704) Sarvarov, F. S. Doctoral Thesis, Novosibirsk, USSR, 1977.

(705) Lendi, K. Chem. Phys. 1977, 20, 135

(706) Kubarev, S. I.; Shustov, A. S. Theor. Exp. Chem. 1980, 16, 418.

(707) Tang, J.; Norris, J. R. Chem. Phys. Lett. 1982, 92, 136.

(708) Salikhov, K. M.; Mikhailov, S. A. Theor. Exp. Chem. 1983 19,508 .

(709) Hoff, A. J.; Hore, P. J. Chem. Phys. Lett. 1984, 108, 104.

(710) Jameson, A. SIAM J. Appl. Math. 1968, 16, 1020

(711) Pedersen, J. B.; Freed, J. H. J. Chem. Phys. 1972, 57, 1004.

(712) Pedersen, J. B.; Freed, J. H. J. Chem. Phys. 1973, 58, 2746.

(713) Pedersen, J. B.; Freed, J. H. J. Chem. Phys. 1974, 61, 1517.

(714) Pedersen, J. B.; Freed, J. H. J. Chem. Phys. 1975, 62, 1790.

(715) Salikhov, K. M.; Sarvarov, F. S.; Sagdeev, R. Z.; Molin, Yu. N. Kinet. Katal. 1975, 16, 279.

(716) Evans, G. T. Mol. Phys, 1976, 31, 777.

(717) Sarvarov, F. S.; Salikhov, K. M. React. Kinet. Catal. Lett. $1976,4,33$

(718) de Kanter, F. J. J.; Kaptein, R.; van Santen, R, A. Chem. Phys. Lett. 1977, 45, 575.

(719) de Kanter, F. J. J.; Kaptein, R. Chem. Phys. Lett. 1978, 58, 340

(720) de Kanter, F. J. J.; Sagdeev, R. Z.; Kaptein, R. Chem. Phys. Lett. 1978, 58, 334 .

(721) Haberkorn, R. Chem. Phys. 1977, 26, 35.

(722) Adrian, F. J.; Monchick, L. J. Chem. Phys. 1979, 71, 2600.

(723) Adrian, F. J.; Monchick, L J Chem. Phys, 1980, 72, 5786.

(724) Zientara, G. P.; Freed, J. H. J. Chem. Phys. 1979, 70, 1359.

(725) Zientara, G. P.; Freed, J. H. J. Phys. Chem. 1979, 83, 3333.

(726) Shushin, A. I. Chem. Phys. Lett. 1982, 85, 562

(727) Mints, R. G.; Pukhov, A. A. Chem. Phys. 1984, 87, 467.

(728) Lüders, K.; Salikhov, K. M. Chem. Phys. 1985, 98, 259.

(729) Shushin, A. I. Mol. Phys. 1986, 58, 1101.

(730) Deutch, J. M. J. Chem. Phys. 1973, 59, 2762

(731) Carslaw, H. S.; Jaeger, J. C. Conduction of Heat in Solids Oxford University Press: London, 1959.

(732) Reference 52, p 91

(733) Closs, G. L. J. Am. Chem. Soc. 1971, 93, 1546.

(734) Avakian, P.; Suna, A. Mater. Res. Bull. 1971, 6, 891.

(735) Kubarev, S. I.; Pshenichnov, E. A.; Shoostov, A. S. Chem. Phys. Lett. 1972, 13, 485.

(736) Ruedin, Y.; Schnegg, P.-A.; Jaccard, C.; Aegerter, M. A. Phys. Status Solidi $B$ : $1972,546,565$.

(737) Kubarev, S. I.; Pshenichnov, E. A.; Shustov, A. S. Theor Exp. Chem. 1976, 12, 329.

(738) Kubarev, S. I.; Sheberstov, S. V.; Shustov, A. S. Chem. Phys. Lett. 1980, 73, 370 .

(739) Movaghar, B.; Ries, B.; Schweitzer, L. Philos. Mag. 1980, B41, 141.

(740) Movaghar, B.; Ries, B.; Schweitzer, L. Philos. Mag. 1980, B41, 159.

(741) Doktorov, A. B.; Anisimov, O. A.; Burshtein, A. I.; Molin, Yu. N. Chem. Phys. 1982, 71,1.

(742) Lersch, W.; Ogrodnik, A.; Michel-Beyerle, M. E. Naturforsch. $1982,37 a, 1454$

(743) Tang, J.; Norris, J. R. Chem. Phys. Lett. 1983, 94, 77.

(744) Hunter, D. A.; Hoff, A. J.; Hore, P.-J. Chem. Phys. Lett. 1987 134,6 .

(745) Lersch, W.; Michel-Beyerle, M. E. Chem. Phys. Lett. 1987, $136,346$.

(746) Petzinger, K. G.; Scalapino, D. J. Phys. Rev. 1973, B8, 266.

(747) Kalckar, F.; Teller, E. Proc. R. Soc. London 1935, A150, 520

(748) Herzberg, G. Molecular Spectra and Molecular Structure (Spectra of Diatomic Molecules, Vol. I); Van NostrandReinhold: New York, 1950; p 139.

(749) Farkes, L. Garbatski, U.J Chem. Phys, 1938, 6, 260

(750) Nielson, S. E.; Dahler, J. S. J. Chem. Phys. 1967, 46, 732.

(751) Harrison, L. G.; McDowell, C. A. Proc. R. Soc. London 1953, A220,77.

(752) Sandler, Y. L. Can. J. Chem. 1954, 32, 249.

(753) Leffler, A. J. J. Chem. Phys. 1965, 43, 4410. 
(754) Ilisca, E. Phys. Rev. Lett. 1970, 24, 797.

(755) Ilisca, E.; Legrand, A. P. Phys. Rev. 1972, B5, 4994.

(756) Atkins, P. W.; Clugston, M. J. Mol. Phys. 1974, 27, 1619.

(757) Ilisca, E.; Gallais, E. Phys. Rev. 1972, B6, 2858.

(758) Ilisca, E.; Debauche, M.; Motchane, J. L. Phys. Rev. 1980, $B 22,687$.

(759) Ilisca, E.; Debauche, M. Surf. Sci. 1984, 144, L449.

(760) Wangness, R. K.; Bloch, F. Phys. Rev. 1953, 89, 728.

(761) Anisimov, O. A.; Molin, Yu. N. High Energy Chem. 1980, 14, 225.

(762) Fedotova, E. Ya.; Sokolik, I. A.; Frankevich, E. L. High Energy Chem. 1980, 14, 261.

(763) Frankevich, E. L.; Fedotova, E. Ya. High Energy Chem. 1980 , 14,355 .

(764) Goldstein, R. A.; Boxer, S. G. Biophys. J. 1987, 51, 937.

(765) Weller, A.; Staerk, H.; Schomburg, H. Acta Phys. Polon. 1987, A71, 707.
(766) Weller, A. In Supramolecular Photochemistry; Balzani, V., Ed.; Reidel: Dordrecht, The Netherlands, 1987; p 343.

(767) Turro, N. J.; Zimmt, M. B.; Gould, I. R. J. Phys. Chem. 1988, $92,433$.

(768) Evans, C.; Ingold, K. U.; Scaiano, J. C. J. Phys. Chem. 1988, $92,1257$.

(769) Willig, F., private communication. Willig, F.; Charle, K.-P. "Spin Dependent Kinetics in Dye Sensitized Charge Carrier Injection" In Modern Aspects of Electrochemistry; Bockris, J. O., Conway, B. E., Eds.; Butterworths, London, in press.

(770) Lendi, K.; Gerber, P.; Labhart, H. Chem. Phys. 1976, 18, 449.

(771) Altwegg, L. Chem. Phys. Lett. 1979, 63, 97.

(772) Ferraudi, G.; Pacheco, M. Chem. Phys. Lett. 1984, 112, 187

(773) Ferraudi, G.; Argüello, G. A.; Frink, M. E. J. Phys. Chem. $1987,91,64$.

(774) Ferraudi, G.; Argüello, G. A. J. Phys. Chem. 1988, 92, 1846.

(775) Steiner, U. E.; Haas, B.-M., unpublished. 